UNIVERSIDADE DE SÃO PAULO

Escola de Engenharia de São Carlos

Departamento de Engenharia de Transportes

Programa de Pós-Graduação em Engenharia de Transportes

Diego de Oliveira Martins

\title{
Monitoramento geodésico de estruturas por meio do método multipolar de aproximações sucessivas e de redes topográficas espaciais
}

Programa de Pós-graduação em Engenharia de Transportes da EESC-USP Exemplar definitivo (corrigido). O exemplar original está disponível na CPG da EESC-USP

São Carlos, 05/09/2019

RESOLUÇÃO CoPGr N 0 6018, DE 13 DE OUTUBRO DE 2011, artigo 5o 

Diego de Oliveira Martins

\section{Monitoramento geodésico de estruturas por meio do método multipolar de aproximações}

\section{sucessivas e de redes topográficas}

\section{espaciais}

Tese de Doutorado submetida à Escola de Engenharia de São Carlos, da Universidade de São Paulo, como parte dos requisitos para a obtenção do título de Doutor em Ciências, Programa de Pós-Graduação em Engenharia de Transportes. Área de Concentração: Infraestrutura de Transportes

Orientador: Professor Associado Irineu da Silva 
AUTORIZO A REPRODUÇÃO TOTAL OU PARCIAL DESTE TRABALHO, POR QUALQUER MEIO CONVENCIONAL OU ELETRÔNICO, PARA FINS DE ESTUDO E PESQUISA, DESDE QUE CITADA A FONTE.

Ficha catalográfica elaborada pela Biblioteca Prof. Dr. Sérgio Rodrigues Fontes da EESC/USP com os dados inseridos pelo(a) autor(a).

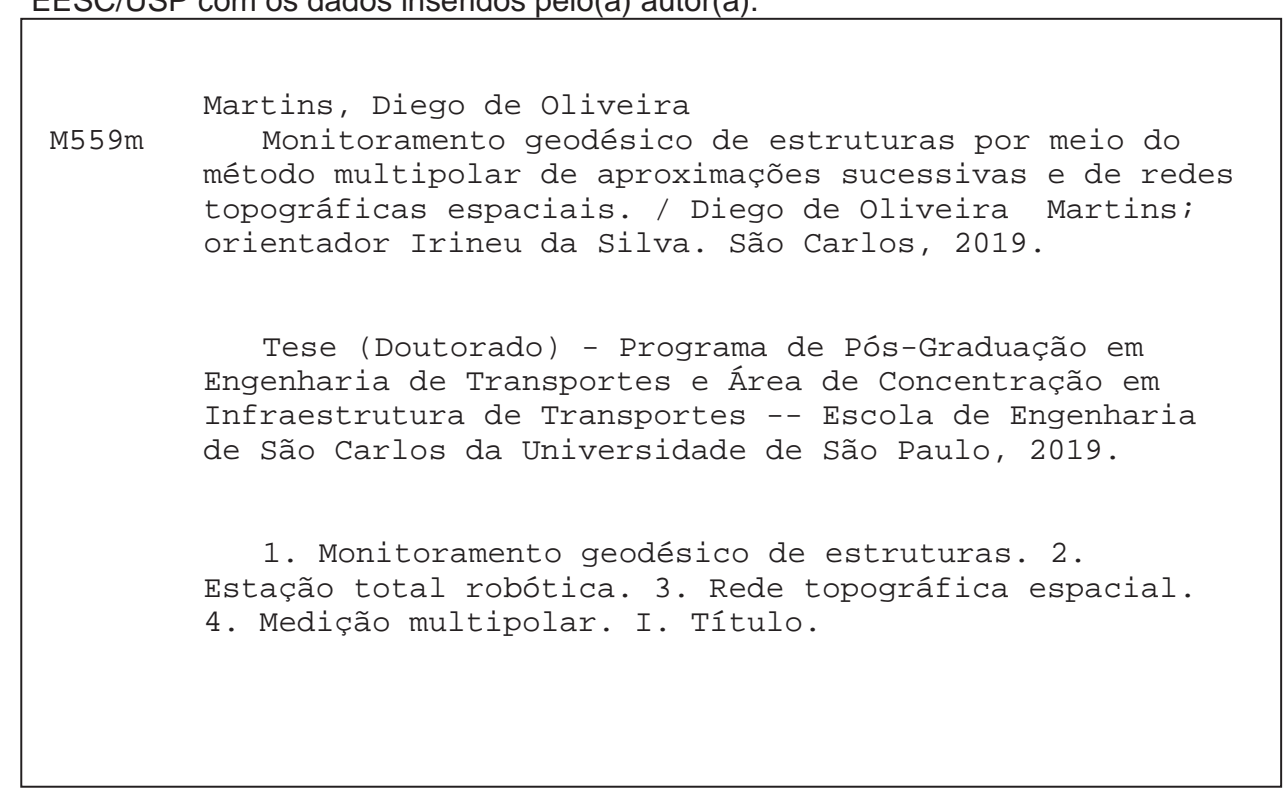

Eduardo Graziosi Silva - CRB - 8/8907 


\section{FOLHA DE JULGAMENTO}

Candidato: Engenheiro DIEGO DE OLIVEIRA MARTINS.

Título da tese: "Monitoramento geodésico de estruturas por meio do método multipolar de aproximações sucessivas e de redes topográficas espaciais".

Data da defesa: 04/04/2019.

Comissão Julgadora:

Prof. Associado Irineu da Silva

(Orientador)

(Escola de Engenharia de São Carlos/EESC)

\section{Resultado:}

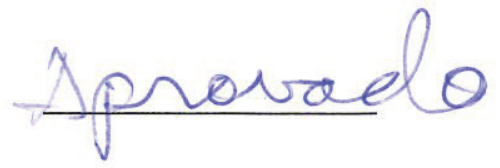

Prof. Associado Edvaldo Simões da Fonseca Junior

AiRous- $\rightarrow$

(Escola Politécnica/EP-USP)

Prof. Dr. João Francisco Galera Monico

A PRO VADO

(Universidade Estadual Paulista "Júlio de Mesquita Filho"/UNESP -

Presidente Prudente)

Prof. Dr. Pedro Luís Faggion

Arrovaro

(Universidade Federal do Paraná/UFPR)

Prof. Dr. Moisés Aparecido do Nascimento

(Universidade Tecnológica Federal do Paraná/UTFPR)

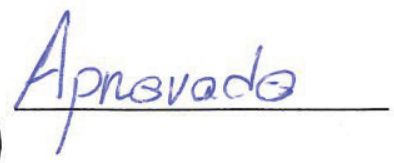

Coordenadora do Programa de Pós-Graduação em Engenharia de Transportes:

Profa. Associada Ana Paula Camargo Larocca

Presidente da Comissão de Pós-Graduação:

Prof. Titular Murilo Araujo Romero 

A Deus, a Jesus Cristo, ao Divino Espírito Santo, a Nossa Senhora da Conceição Aparecida e à Minha Família. 



\section{Agradecimentos}

Primeiramente agradeço à diviníssima trindade, a Nossa Senhora da Conceição Aparecida e toda minha família.

Aos meus pais Lauriberto José Martins e Arlete de Oliveira Martins por tudo o que realizam em minha vida.

Aos meus irmãos Ariel de Oliveira Martins e Carolina de Oliveira Martins, pelo amor, alegria e união.

A maravilhosa sobrinha Maria Luiza Martins Gerevini.

À minha amada Ana Carolina Pereira, pelo companheirismo, amor, compreensão, força e carinho.

Aos meu tios Altamir Martins de Oliveira e Marta Jurado Garcia, pela fraternidade e amparo.

Ao meu primo Gabriel Jurado Martins de Oliveira, pela parceria, amizade e por tudo o que fez para o êxito desta pesquisa.

Ao meu primo Maurício Perroni, por fornecer e colaborar com a instalação das tendas de proteção dos instrumentos utilizados.

Ao meu orientador Professor Associado Irineu da Silva, pela amizade, conselhos, confiança e paciência.

Ao STT, pela oportunidade e apoio na realização da pesquisa.

Aos professores e funcionários do STT, pela contribuição para a minha formação.

A Escola de Engenharia de São Carlos (EESC), pela sinergia oferecida. 
A Prefeitura do Campus USP de São Carlos (PUSP-SC), por conceder acesso ao Campus 2, oferecendo infraestrutura, segurança e apoio ao desenvolvimento desta pesquisa.

A Cilene de Cassia Garcia, chefe da Divisão de Espaço Físico (DVEF), por viabilizar acesso a todo espaço necessário, pela colaboração na materialização da base 6 no edifício Polo Terra e por oferecer profissionais de segurança do trabalho para execução do processo de monitoramento.

Ao Instituto de Física de São Carlos (IFSC), por conceder espaço em seu edíficio no Campus 2, no qual foi materializada a base 1 e o CORE do sistema de monitoramento geodésico de estruturas proposto.

Ao Instituto de Química de São Carlos (IQSC), por conceder espaço em seu edíficio no Campus 2, no qual foi materializada a base 2 .

Ao Orlando Antonio Picchi Júnior e todos os técnicos, funcionários e profissionais de segurança que trabalham no Campus 2, por todo apoio e suporte em todo a execução dos experimentos.

Aos inestimáveis amigos Mariovaldo e Marinaldo Marchetti, por toda a forma de cooperação no desenvolvimento do sistema de monitoramento geodésico de estruturas proposto. E consequentemente, a Braspowertech Soluções Tecnológicas, pelas certificações de calibração das estações totais robóticas e por todos os serviços executados com excelência.

Aos caros amigos Patrick Pires e Gleyson Marquesin, da PCP Engenharia, pela parceria e apoio no desenvolvimento desta pesquisa.

Aos prezados amigos Carlos Roberto Catarino Júnior e Jaqueline Muniz Cabrera da Interchip Suprimentos para Informática, pelo suporte em toda a realização das instalações do sistema operacional.

Ao nobre amigo Norberto Yukio Yagasaki, por tudo o que fez por mim e pelo desenvolvimento desta pesquisa.

Ao querido amigo José Vanderlei Scansani de Mello, pela força na espacialização, montagem e desmontagem da instrumentação da pesquisa.

Ao grande amigo Marco Antônio, pelo apoio na instrumentação desta pesquisa.

A amável Professora Marlene Carolina de Souza, por tudo o que faz pela educação e por seus alunos.

A todo o grupo de estudo do Professor Irineu da Silva, ao Francisco Roza de Moraes, ao Guilherme Poleszuk dos Santos Rosa, a Maria Lígia Chuerubim, ao Jefferson Teixeira Brasil M. Junior e ao Sérgio Roberto de Oliveira.

A todos os colegas e amigos que colaboraram de forma direta e indireta, para a realização desta pesquisa. 
Ao Conselho Nacional de Desenvolvimento de Científico e Tecnológico (CNPq), pelo amparo a esta pesquisa. 

"A boa vontade é nosso recurso de cada hora."

(Jesus Cristo) 



\section{Resumo}

Martins, D. O. Monitoramento geodésico de estruturas por meio do método multipolar de aproximações sucessivas e de redes topográficas espaciais. $238 \mathrm{p}$. Tese de Doutorado Escola de Engenharia de São Carlos, Universidade de São Paulo, 2019.

O monitoramento de estruturas por meio de métodos geodésicos é uma prática já consagrada nos meios técnico e científico. Por muito tempo, eles se basearam em técnicas de triangulação, trilateração e nivelamento geométrico de precisão. Mais recentemente, com o advento das Estações Totais Robóticas (ETR) e as evoluções tecnológicas do Global Navigation Satellite System (GNSS), passou-se também a utilizar o método da irradiação topográfica tridimensional e o processamento GNSS, em modo Real Time Kinematic (RTK), como opções para a automatização dos processos de monitoramento geodésico. No caso do uso de uma estação total robótica, o movimento da estrutura é determinado por meio de medições angulares e de distâncias inclinadas, geralmente, realizadas com um instrumento instalado sobre um pilar de referência. $\mathrm{O}$ movimento percebido é calculado em função da variação dos valores das coordenadas espaciais $(X, Y, Z)$ dos pontos monitorados. A determinação das coordenadas, neste caso, é realizada empregando-se o método de medição polar, em que cada ponto é observado individualmente, a partir de um único pilar de referência. Os resultados obtidos com esse método de medição topográfica têm-se mostrado eficientes, porém, pouco robustos devido à inexistência de redundância nas medições. Por esta razão, alguns pesquisadores vêm buscando alternativas e propondo métodos de medições multipolares, em que as coordenadas espaciais dos pontos observados são determinadas em função de redes topográficas espaciais, em que cada ponto é observado por mais de uma estação de referência. A este respeito, existem vários modelos 
matemáticos que podem ser aplicados para a determinação das coordenadas espaciais, os quais estão descritos e avaliados nesta pesquisa. Entre eles, cita-se o método de medição multipolar iterativo de aproximações sucessivas, desenvolvido pelo autor, e que se baseia em um modelo matemático de análise numérica, que gera, a partir de uma aproximação inicial, uma sequência de soluções que converge para a melhor aproximação das coordenadas do alvo monitorado. Dado ao fato de os ângulos e as distâncias serem observados simultaneamente a partir de vários pilares de medição, os quais estão sujeitos a verificações de estabilidade e controle, o modelo proposto possui redundância de observações, o que o torna mais robusto e eficiente que seu predecessor. Em consequência disso, é possível aplicar métodos estatísticos para as avaliações das possíveis movimentações dos elementos estruturais e os alarmes gerados pelo sistema se tornam mais confiáveis para informarem eventuais situações de riscos. A aplicação deste método de medição multipolar parte de uma rede topográfica espacial calculada e ajustada, para a determinação simultânea das coordenadas espaciais $(X, Y, Z)$ de cada ponto observado, diferentemente do que se pode encontrar disponível na literatura atual, em que os componentes horizontais e verticais são determinados separadamente. Nesse contexto, esta tese tem como objetivo desenvolver um sistema de monitoramento geodésico de estruturas com o uso de várias estações totais robóticas.

Palavras-chave: Monitoramento geodésico de estruturas. Estação total robótica. Rede topográfica espacial. Medição multipolar. 


\section{Abstract}

Martins, D. O. Geodetic monitoring of structures by means of the iterative multipolar measurement method of successive approximations and spatial topographic networks. 238 p. Ph.D. Thesis - São Carlos School of Engineering, University of São Paulo, 2019.

The monitoring of structures from geodetic methods is a practice already consecrated in the technical and scientific means. For a long time, they have relied on triangulation techniques, trilateration, and precision geometric leveling. More recently, with the advent of Robotic Total Stations (RTS) and technological developments of Global Navigation Satellite System (GNSS), three-dimensional topographic irradiation method and GNSS processing in the Real Time Kinematic (RTK), emerged as options for automation of geodetic monitoring processes. In the case of the use of an robotic total station, the movement of the structure is determined through angular measurements and inclined distances, generally performed with an instrument installed on a reference pillar. The perceived movement is calculated as a function of the variation of the spatial coordinates $(X, Y, Z)$ values of the monitored points. The determination of the coordinates in this case is performed using polar measurement method, where each point is observed individually from a single monitoring station. The results obtained with this method of topographic measurement have been shown to be efficient, but not robust due to the lack of redundancy in the measurements. For this reason, some researchers have been searching for alternatives and proposing methods of multipolar measurements, in which the spatial coordinates of the observed points are determined as a function of spatial geodesic networks, where each point is observed by more than one reference station. To this subject, there are several mathematical models that can be applied to determine spatial coordinates, which are 
described and evaluated in this research. Among them, the iterative multipolar method of successive approximations developed by the author, is based on a mathematical model of numerical analysis, which generates, from an initial approximation, a sequence of solutions that converges to a better approximation of the coordinates of the monitored target. Given the fact that angles and distances are observed simultaneously from several measurement pillars, which are subject to stability and control checks, the proposed model has redundancy of observations, which makes it more robust and efficient than its predecessor. As consequence, it is possible to apply statistical methods for evaluating the possible movements of the structural elements and the alarms generated by the system become more reliable to inform possible situations of risks. The application of this multipolar measurement method start from a spatial topographic network calculated and adjusted for the simultaneous determination of the spatial coordinates $(X, Y, Z)$ of each observed point, differently from what can be found in the current literature, where horizontal components are determined separately. In this context, this thesis aims to develop a system of geodetic monitoring of structures with the use of several robotic total stations.

Keywords: Geodetic monitoring of structures. Robotic total station. Spatial topographic neiltwork. Multipolar measurement. 


\section{Lista de ilustrações}

Figura 2.1 Componentes de um sistema de monitoramento geodésico. . . . . . . . . . 36

Figura 2.2 Fluxograma de operações de monitoramento. . . . . . . . . . . . . . . . . 38

Figura 2.3 Instalação de monitoramento contínuo, com o uso de estações totais robóticas. 42

Figura 3.1 Estações Totais Robóticas utilizadas. . . . . . . . . . . . . . . . . . . . 50

Figura 3.2 Bases nivelantes de precisão utilizadas. . . . . . . . . . . . . . . 51

Figura 3.3 Carrier de precisão utilizado. . . . . . . . . . . . . . . . . 51

Figura 3.4 Prismas refletores utilizados. . . . . . . . . . . . . . . . . 52

Figura 3.5 Composição de base nivelante, carrier e prisma refletor. . . . . . . . . . . 52

Figura 3.6 Tripés industriais da rede e de monitoramento. . . . . . . . . . . . . . 53

Figura 3.7 Sensor metereológico. . . . . . . . . . . . . . . . . . 56

Figura 3.8 Elementos descritivos do braço robótico. . . . . . . . . . . . . 56

Figura 3.9 Alcance e intervalo de carga dinâmica braço robótico. . . . . . . . . . . . 57

Figura 3.10 Dimensões do braço robótico em milímetros. . . . . . . . . . . . . . . 57

Figura 3.11 Especificações do uArm Swift Pro. . . . . . . . . . . . . . . . . . 58

Figura 3.12 Trava universal presa ao end effector. . . . . . . . . . . . . . . . . 58

Figura 3.13 Trava universal, segurando um pino de acoplagem. . . . . . . . . . . . . . 59

Figura 3.14 Sistema para controle do alvo. . . . . . . . . . . . . . . . . . . 59

Figura 3.15 Caixas de comunicação para as estações totais robóticas. . . . . . . . . . 60

Figura 3.16 Antenas e acessórios para transmissão de dados. . . . . . . . . . . . . . 61

Figura 3.17 Comunicação da intranet. . . . . . . . . . . . . . . . . 62

Figura 4.1 Rede de Triangulação. . . . . . . . . . . . . . . . . . . . . . 67

Figura 4.2 Rede de Trilateração. . . . . . . . . . . . . . . . . . . . . 67 
Figura 4.3 Rede de triangulateração. . . . . . . . . . . . . . . . . . . . . . 68

Figura 4.4 Poligonal topográfica . . . . . . . . . . . . . . . . . . . . . . . . 69

Figura 4.5 Princípio básico de um nivelamento geométrico. . . . . . . . . . . . 70

Figura 4.6 Relação geométrica de um nivelamento trigonométrico. . . . . . . . . . . 71

Figura 4.7 Rede topográfica espacial de referência absoluta. . . . . . . . . . . . . . . 72

Figura 4.8 Relação geométrica de uma recessão espacial. . . . . . . . . . . . . . . . . . 73

Figura 5.1 Observação de uma distância inclinada . . . . . . . . . . . . . . . . . . 84

Figura 5.2 Observação de um ângulo vertical zenital. . . . . . . . . . . . . . . . . . 87

Figura 5.3 Observação de um ângulo vertical de altura. . . . . . . . . . . . . . . . . 92

Figura 5.4 Relação trigonométrica de uma observação espacial. . . . . . . . . . . . . . 96

Figura 5.5 Relação entre azimute $A z_{i P}^{C}$ e ângulo $\alpha_{i P}^{C}$ a serem calculados. . . . . . . . . . . 98

Figura 5.6 Observação de um ângulo horizontal azimutal. . . . . . . . . . . . . . . . . 99

Figura 5.7 Relação entre um ângulo horizontal interno e dois azimutes. . . . . . . . . . 103

Figura 6.1 Medição polar simples. . . . . . . . . . . . . . . . . . . . . . . . 110

Figura 6.2 Interseção perfeita. . . . . . . . . . . . . . . . . . . . . . . 110

Figura 6.3 Interseção na prática. . . . . . . . . . . . . . . . . . . 110

Figura 6.4 Medição polar e multipolar. . . . . . . . . . . . . . . . . . . . . 112

Figura 6.5 Relações geométricas do método polar. . . . . . . . . . . . . . . . . . 112

Figura 6.6 Representação geométrica do método de interseção plana. . . . . . . . . . . 116

Figura 6.7 Representação da discrepância do método de interseção espacial trigonométrico . . . . . . . . . . . . . . . . . . . . . 116

Figura 6.8 Relações geométricas do ponto médio da interseção espacial. . . . . . . . . 118

Figura 6.9 Pontos $P_{1}$ e $P_{2}$ e projeções $r$ e $s$ da interseção espacial pelo ponto médio. . . 119

Figura 6.10 Geometria da interseção espacial pelas distâncias mínimas. . . . . . . . . . 121

Figura 6.11 Relações geométricas da interseção espacial analítica. . . . . . . . . . . . . 130

Figura 6.12 Relação geométrica para azimute. . . . . . . . . . . . . . . . . . . 133

Figura 6.13 Relação geométrica ângulo vertical de altura. . . . . . . . . . . . . . . . 135

Figura 7.1 Relações geométricas para obtenção das equações das distâncias. . . . . . . 142

Figura 7.2 Representação do processo iterativo, com aproximações sucessivas para duas estações totais robóticas. . . . . . . . . . . . . . . . . . . 147

Figura 7.3 Representação do processo iterativo, com aproximações sucessivas para três estações totais robóticas. . . . . . . . . . . . . . . . . . . . . . . 154

Figura 8.1 Localização e espacialização dos elementos no Campus 2 da USP São Carlos. 158

Figura 8.2 Base 1 (IFSC) . . . . . . . . . . . . . . . . . . . . . . 160

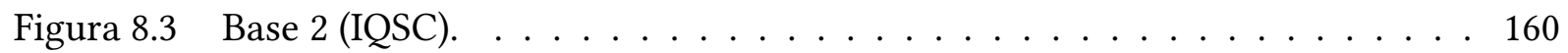

Figura 8.4 Base 6 (Polo Terra). . . . . . . . . . . . . . . . . . . . . . 161

Figura 8.5 Localização e materialização das bases 3 , 4 e $5 \quad \ldots \ldots$. . . . . . . . . 161 
Figura 8.6 Rede topográfica espacial e elipse dos erros. . . . . . . . . . . . . . . . 165

Figura 8.7 Rede topográfica espacial de referência absoluta e controle. . . . . . . . . . 166

Figura 8.8 Localização e operação do robô. . . . . . . . . . . . . . . . . . . . . 166

Figura 8.9 Esquema da sequência lógica do processo de monitoramento. . . . . . . . . 168

Figura 8.10 Layout do sistema integrado, com três estações totais robóticas. . . . . . . 168

Figura 8.11 Diagrama do sistema operacional de cada ambiente. . . . . . . . . . . . . . 169

Figura 8.12 Esquema de comunicação do sistema. . . . . . . . . . . . . . . . . 170

Figura 8.13 Layout do link de comunicação. . . . . . . . . . . . . . . . . . . . . . . . 170

Figura 8.14 Concepção do sistema de gerenciamento proposto. . . . . . . . . . . . . . . 171

Figura 8.15 Etapas do sistema de gerenciamento de dados. . . . . . . . . . . . . . . . 172

Figura 8.16 Criação do template do sistema. . . . . . . . . . . . . . . . . . . . . . 173

Figura 8.17 Input dos dados brutos no sistema. . . . . . . . . . . . . . . . . . . . . . 174

Figura 8.18 Gerenciamento dos dados do sistema. . . . . . . . . . . . . . . . . . . . 174

Figura 8.19 Posição do atributo no vetor de matrizes (mapping). . . . . . . . . . . . . . 174

Figura 8.20 Output de dados processados no sistema. . . . . . . . . . . . . . . . 175

Figura 9.1 Variação da temperatura . . . . . . . . . . . . . . . . . . . . . . . 180

Figura 9.2 Variação da pressão atmosférica. . . . . . . . . . . . . . . . . . . . . . . 180

Figura 9.3 Variação das coordenadas espaciais do alvo controlado pelo braço robótico. 182

Figura 9.4 Posições das coordenadas $X_{P}, Y_{P}$ e $Z_{P}$ (braço robótico) . . . . . . . . . . . 182

Figura 9.5 Variação das coordenadas espaciais do método polar com média. . . . . . . 184

Figura 9.6 Posições das coordenadas $X_{P}, Y_{P}$ e $Z_{P}$ (polar com média). . . . . . . . . . . 184

Figura 9.7 Variação das coordenadas espaciais do método polar com ajustamento. . 185

Figura 9.8 Posições das coordenadas $X_{P}, Y_{P}$ e $Z_{P}$ (polar com ajustamento). . . . . . . . 186

Figura 9.9 Variação das coordenadas espaciais do método de interseção espacial trigonométrico. . . . . . . . . . . . . . . . . . . . . . . 187

Figura 9.10 Posições das coordenadas $X_{P}, Y_{P}$ e $Z_{P}$ (interseção espacial trigonométrico). 187

Figura 9.11 Variação das coordenadas espaciais do método do ponto médio da interseção espacial. . . . . . . . . . . . . . . . . . . . . . . . . . . . . . . 189

Figura 9.12 Posições das coordenadas $X_{P}, Y_{P}$ e $Z_{P}$ (ponto médio da interseção espacial). 189

Figura 9.13 Variação das coordenadas espaciais do método das distâncias mínimas. . 190

Figura 9.14 Posições das coordenadas $X_{P}, Y_{P}$ e $Z_{P}$ (distâncias mínimas). . . . . . . . . 191

Figura 9.15 Variação das coordenadas espaciais do método das distâncias mínimas com ajustamento.

Figura 9.16 Posições das coordenadas $X_{P}, Y_{P}$ e $Z_{P}$ (distâncias mínimas com ajustamento). 192

Figura 9.17 Variação das coordenadas espaciais do método de interseção espacial analítica. . . . . . . . . . . . . . . . . . . . . . 194

Figura 9.18 Posições das coordenadas $X_{P}, Y_{P}$ e $Z_{P}$ (interseção espacial analítica). . . . . 194 
Figura 9.19 Variação das coordenadas espaciais do método da minimização dos deslocamentos em função dos azimutes, dos ângulos verticais e das distâncias inclinadas medidas (relação geométrica). . . . . . . . . . . . . 196

Figura 9.20 Posições das coordenadas $X_{P}, Y_{P}$ e $Z_{P}$ (minimização com relação geométrica). 196

Figura 9.21 Variação das coordenadas espaciais do método da minimização dos resíduos das funções trigonométricas em função dos azimutes, dos ângulos verticais e distâncias inclinadas medidas (relação analítica). . . . . . . . . . . . . 198

Figura 9.22 Posições das coordenadas $X_{P}, Y_{P}$ e $Z_{P}$ (minimização com relação analítica). 198

Figura 9.23 Variação das coordenadas espaciais do método iterativo de aproximações sucessivas, para determinação de coordenadas espaciais, considerando duas estações totais robóticas. . . . . . . . . . . . . . . . . 200

Figura 9.24 Posições das coordenadas $X_{P}, Y_{P}$ e $Z_{P}$ (iterativo com 2 ETR). . . . . . . . 200

Figura 9.25 Variação das coordenadas espaciais do método iterativo de aproximações sucessivas, para determinação de coordenadas espaciais, considerando três estações totais robóticas. . . . . . . . . . . . . . . . . . . 202

Figura 9.26 Posições das coordenadas $X_{P}, Y_{P}$ e $Z_{P}$ (iterativo com 3 ETR). . . . . . . . . 202

Figura 9.27 Posições das coordenadas $X_{P}, Y_{P}$ e $Z_{P}$ do braço robótico em todo o experimento. . . . . . . . . . . . . . . . . . 203

Figura 9.28 Posições das coordenadas $X_{P}, Y_{P}$ e $Z_{P}$ do método iterativo com 2 ETR em todo o experimento. . . . . . . . . . . . . . . . . 203

Figura 9.29 Posições das coordenadas $X_{P}, Y_{P}$ e $Z_{P}$ do método iterativo com 3 ETR em todo o experimento. . . . . . . . . . . . . . . 204

Figura 9.30 Convergência das coordenadas $X_{P}, Y_{P}$ e $Z_{P}$ do método iterativo com 2 ETR. 204

Figura 9.31 Convergência das coordenadas $X_{P}, Y_{P}$ e $Z_{P}$ do método iterativo com 3 ETR. 204

Figura 9.32 Análise comparativa da posição espacial $X_{P} \ldots \ldots \ldots$. . . . . . . . 214

Figura 9.33 Análise comparativa da posição espacial $Y_{P} \ldots \ldots$. . . . . . . . . . . . 215

Figura 9.34 Análise comparativa da posição espacial $Z_{P}$. . . . . . . . . . . . . . 215

Figura 9.35 Acurácia obtida em $X_{P} \ldots \ldots \ldots \ldots \ldots \ldots \ldots \ldots$

Figura 9.36 Acurácia obtida em $Y_{P} \ldots \ldots \ldots \ldots \ldots \ldots \ldots \ldots$

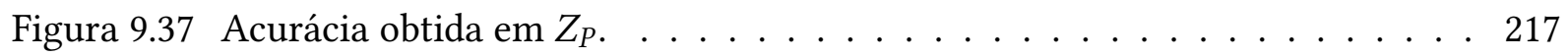

Figura A.1 Certificado de calibração da TCA1201+. . . . . . . . . . . . . . . . 236

Figura A.2 Certificado de calibração da TCRP1201+R300 . . . . . . . . . . . . . . 237

Figura A.3 Certificado de calibração da TCRA1201+R400 . . . . . . . . . . . . . . 238 


\section{Lista de tabelas}

Tabela 2.1 Requisitos de precisões para obras de engenharia civil (95\% RMS) . . . . . 39

Tabela 2.2 Tipos de comunicação em função do objeto de monitoramento. . . . . . . . 41

Tabela 3.1 Características técnicas básicas de estações totais robóticas para monitoramento. ..................... . . . 47

Tabela 5.1 Estrutura da Matriz A para distância inclinada. . . . . . . . . . . . . . 86

Tabela 5.2 Estrutura da Matriz A para ângulo vertical zenital. . . . . . . . . . . . . . 91

Tabela 5.3 Estrutura da Matriz A para ângulo vertical de altura. . . . . . . . . . . . . . 95

Tabela 5.4 Relação entre quadrante, $C$, azimute e o ângulo $\alpha$. . . . . . . . . . . . 97

Tabela 5.5 Estrutura da Matriz A para ângulo horizontal azimutal. . . . . . . . . . . 102

Tabela 5.6 Estrutura da Matriz A para ângulo horizontal interno. . . . . . . . . . . . 106

Tabela 8.1 Coordenadas espaciais arbitradas para a base 1 . . . . . . . . . . . . 161

Tabela 8.2 Dados observados para o cálculo da rede topográfica espacial. . . . . . . . 163

Tabela 8.3 Coordenadas aproximadas das bases. . . . . . . . . . . . . . . . 164

Tabela 8.4 Coordenadas e precisões ajustadas das bases. . . . . . . . . . . . . . . 164

Tabela 8.5 Teste do $\chi^{2}$ para verificação e análise da confiabilidade da rede espacial. . . 165

Tabela 9.1 Dados de entrada da amostra 1. . . . . . . . . . . . . . 178

Tabela 9.2 Dados de entrada da amostra 2. . . . . . . . . . . . . . . . . 178

Tabela 9.3 Dados de entrada da amostra 3. . . . . . . . . . . . . . . . . . 179

Tabela 9.4 Dados de entrada da amostra 4. . . . . . . . . . . . . . . . . . . . . 179

Tabela 9.5 Coordenadas espaciais posicionadas pelo robô na amostra 1 . . . . . . . . 181

Tabela 9.6 Coordenadas espaciais posicionadas pelo robô na amostra $2 \ldots$. . . . . . . 181 
Tabela 9.7 Coordenadas espaciais posicionadas pelo robô na amostra 3 . . . . . . . . 181

Tabela 9.8 Coordenadas espaciais posicionadas pelo robô na amostra 4 . . . . . . . . 182

Tabela 9.9 Coordenadas espaciais determinadas pela média do método polar. . . . . . 183

Tabela 9.10 Coordenadas espaciais determinadas pelo método polar com ajustamento. 185

Tabela 9.11 Coordenadas espaciais determinadas pelo método de interseção espacial trigonométrico. . . . . . . . . . . . . . . . . . 186

Tabela 9.12 Coordenadas espaciais determinadas pelo método do ponto médio da interseção espacial. . . . . . . . . . . . . . . . . . . . 188

Tabela 9.13 Coordenadas espaciais determinadas pelo método das distâncias mínimas. 190

Tabela 9.14 Coordenadas espaciais determinadas pelo método das distâncias mínimas com ajuste das observações. . . . . . . . . . . . . . . . . . . . . . . . . 191

Tabela 9.15 Coordenadas espaciais determinadas pelo método de interseção espacial analítica.

Tabela 9.16 Coordenadas espaciais determinadas pelo método da minimização dos deslocamentos em função dos azimutes, dos ângulos verticais e das distâncias inclinadas medidas (relação geométrica). . . . . . . . . . . . . . . .

Tabela 9.17 Coordenadas espaciais determinadas pelo método da minimização dos resíduos das funções trigonométricas em função dos azimutes, dos ângulos verticais e distâncias inclinadas medidas (relação analítica) . . . . . . . . . .

Tabela 9.18 Coordenadas espaciais determinadas pelo método iterativo de aproximações sucessivas, para determinação de coordenadas espaciais, considerando duas estações totais robóticas. . . . . . . . . . . . . . . . . .

Tabela 9.19 Coordenadas espaciais determinadas pelo método iterativo de aproximações sucessivas, para determinação de coordenadas espaciais, considerando três estações totais robóticas. . . . . . . . . . . . . . . . . . . . . 201

Tabela 9.20 Deslocamentos e acurácias obtidas pela média do método polar. . . . . . . 205

Tabela 9.21 Deslocamentos e acurácias obtidas pelo método polar com ajuste das observações. . . . . . . . . . . . . . . . . . 206

Tabela 9.22 Deslocamentos e acurácias obtidas pelo método de interseção espacial trigonométrico. . . . . . . . . . . . . . .

Tabela 9.23 Deslocamentos e acurácias obtidas pelo método do ponto médio da interseção espacial. . . . . . . . . . . . . . . . . . . . 207

Tabela 9.24 Deslocamentos e acurácias obtidas pelo método das distâncias mínimas. . . 208

Tabela 9.25 Deslocamentos e acurácias obtidas pelo método das distâncias mínimas com ajuste das observações. . . . . . . . . . . . . . . . . 208

Tabela 9.26 Deslocamentos e acurácias obtidas pelo método de interseção espacial analítica. 
Tabela 9.27 Deslocamentos e acurácias obtidas pelo método da minimização dos deslocamentos em função dos azimutes, dos ângulos verticais e das distâncias inclinadas medidas (relação geométrica). . . . . . . . . . . . . . . . 210

Tabela 9.28 Deslocamentos e acurácias obtidas pelo método da minimização dos resíduos das funções trigonométricas em função dos azimutes, dos ângulos verticais e distâncias inclinadas medidas (relação analítica) . . . . . . . . . . 211

Tabela 9.29 Deslocamentos e acurácias obtidas pelo método iterativo de aproximações sucessivas, para determinação de coordenadas espaciais, considerando duas estações totais robóticas. . . . . . . . . . . . . . . . . . . . . . 212

Tabela 9.30 Deslocamentos e acurácias obtidas pelo método iterativo de aproximações sucessivas, para determinação de coordenadas espaciais, considerando três estações totais robóticas. . . . . . . . . . . . . . . . . . . . . . . 213 



\section{Sumário}

$1 \quad$ MONITORAMENTO DE ESTRUTURAS 27

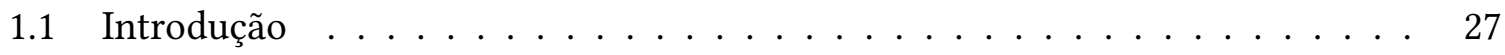

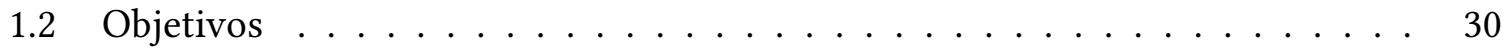

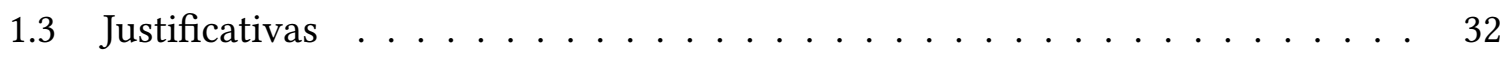

1.4 Estrutura da tese . . . . . . . . . . . . . . . 33

$2 \quad$ MONITORAMENTO GEODÉSICO DE ESTRUTURAS 35

2.1 Sistemas de monitoramento geodésicos de estruturas . . . . . . . . . . . . 35

2.1 .1 Subsistema de medição $\ldots \ldots \ldots$. . . . . . . . . . 36

2.1.2 Subsistema de comunicação . . . . . . . . . . . . . . . . . . . 36

2.1.3 Subsistema de processamento . . . . . . . . . . . . . . 36

2.2 Projetos de monitoramento geodésico de estruturas . . . . . . . . . . . . 37

2.2.1 Estudo do objeto de monitoramento . . . . . . . . . . . . 37

$2.2 .2 \quad$ Plano de medição $\ldots \ldots \ldots$. . . . . . . . . . . . . . 38

2.2.3 Plano de instrumentação . . . . . . . . . . . . . . . . . . . . . 39

2.2.4 Plano de comunicação, de gestão e de transferência de dados . . . . . 41

2.2.5 Plano de periodicidade e monitoramento . . . . . . . . . . . . 42

2.2.6 Plano de processamento, avaliação dos dados e alarme . . . . . . . . . 43 
3 INSTRUMENTOS UTILIZADOS NO MONITORAMENTO GEODÉSICO DE ES$\begin{array}{ll}\text { TRUTURAS } & 45\end{array}$

3.1 Instrumentos utilizados no subsistema de medição . . . . . . . . . . . . . 46

3.1.1 Estação total robótica . . . . . . . . . . . . . . . . . . . 46

$3.1 .2 \quad$ Base nivelante e carrier . . . . . . . . . . . . . . . . 51

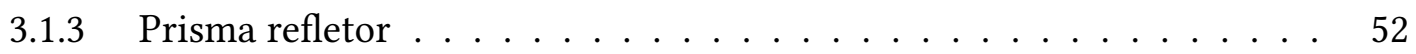

3.1 .4 Tripé industrial $\ldots \ldots \ldots \ldots \ldots \ldots$

3.1.5 Sensor metereológico ....................... 54

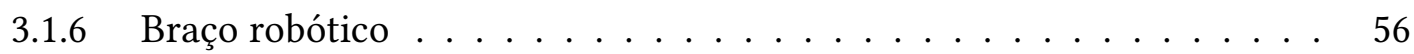

3.2 Instrumentos utilizados no subsistema de comunicação . . . . . . . . . . . 60

3.2 .1 Caixa de comunicação . . . . . . . . . . . . . . . . . 60

3.2.2 Transmissão de dados . . . . . . . . . . . . . . . . . . . . . 61

3.3 Instrumentos utilizados no subsistema de processamento e alarme . . . . . 62

3.3.1 Software comercial . . . . . . . . . . . . . . 62

3.3.2 Software aberto..................... 62

4 TÉCNICAS DE MEDIÇÃO PARA O MONITORAMENTO GEODÉSICO DE ES$\begin{array}{ll}\text { TRUTURAS } & 65\end{array}$

4.1 Técnicas de medições angulares e lineares $\ldots \ldots \ldots$. . . . . . . . . . . . 65

4.2 Determinação planimétrica da rede . . . . . . . . . . . . . . . . 66

4.2.1 Rede de Triangulação . . . . . . . . . . . . . . . . . . 66

4.2 .2 Rede de Trilateração . . . . . . . . . . . . . . . . . . . . . . . 67

4.2 .3 Rede de Triangulateração . . . . . . . . . . . . . . . . . . 68

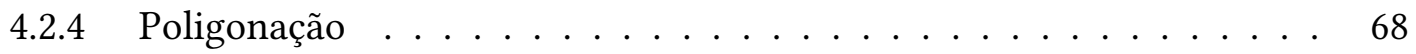

4.3 Determinação altimétrica da rede . . . . . . . . . . . . . . . . . . . 69

4.3.1 Nivelamento geométrico . . . . . . . . . . . . . . . 69

4.3.2 Nivelamento trigonométrico . . . . . . . . . . . . . . . . 71

4.4 Determinação da estabilidade da rede . . . . . . . . . . . . . . . 72

4.4.1 Rede topográfica espacial de referência absoluta . . . . . . . . . . 72

4.4 .2 Recessão espacial . . . . . . . . . . . . . . . . . . 73

4.5 Ajustamento das observações . . . . . . . . . . . . . . . . . . . . 74 
4.5.1 Método paramétrico . . . . . . . . . . . . . . . . . . 74

4.6 Avaliação dos resultados . . . . . . . . . . . . . . . . . . 78

4.6.1 Teste qui-quadrado . . . . . . . . . . . . . . . . 79

4.6 .2 Elipse de erro . . . . . . . . . . . . . . . . . . . . 80

5 TÉCNICAS DE MEDIÇÕES ANGULARES E LINEARES PARA A REDE TOPOGRÁFICA ESPACIAL

5.1 Equação de observação para distância inclinada . . . . . . . . . . . . . . . . 83

5.2 Equação de observação para ângulo vertical zenital . . . . . . . . . . . . . . . 87

5.3 Equação de observação para ângulo vertical de altura . . . . . . . . . . . . . . 91

5.4 Cálculo de azimute em função de coordenadas espaciais . . . . . . . . . . . 95

5.5 Equação de observação para ângulo horizontal azimutal . . . . . . . . . . . . 97

5.6 Equação de observação para ângulo horizontal interno . . . . . . . . . . . . . 102

6 MÉTODOS DE DETERMINAÇÃO DE COORDENADAS ESPACIAIS 109

6.1 Método polar . . . . . . . . . . . . . . . . . . . . 112

6.2 Métodos multipolares sem medições de distâncias . . . . . . . . . . . . . 115

6.2.1 Método de interseção espacial trigonométrico . . . . . . . . . . . . 115

6.2.2 Método do ponto médio da interseção espacial . . . . . . . . . . . . 118

6.2 .3 Método das distâncias mínimas . . . . . . . . . . . . . . . . . . . . 120

6.2.4 Método das distâncias mínimas com ajuste das observações . . . . . . 128

6.2.5 Método de interseção espacial analítica . . . . . . . . . . . . . . . 130

6.3 Métodos multipolares com medições de distâncias ～. . . . . . . . . . . . . . 132

6.3.1 Métodos de minimização dos ângulos e das distâncias medidas . . . . 132

6.3.2 Método da minimização dos deslocamentos em função dos azimutes, dos ângulos verticais e das distâncias inclinadas (relação geométrica) 133

6.3.3 Método da minimização dos resíduos das funções trigonométricas em função dos azimutes, dos ângulos verticais e das distâncias inclinadas (relação analítica) . . . . . . . . . . . . . . . . . . . . 138

7 MÉTODO ITERATIVO PARA DETERMINAÇÃO DE COORDENADAS ESPACIAIS 141

7.1 Método iterativo de aproximações sucessivas, para determinação de coordenadas espaciais, considerando duas estações totais robóticas . . . . . . . . . . 142 
7.2 Método iterativo de aproximações sucessivas, para determinação de coordenadas espaciais, considerando três estações totais robóticas . . . . . . . . 150

$8 \quad$ SISTEMA DE MONITORAMENTO GEODÉSICO PROPOSTO 157

8.1 Descrição do local de estudo . . . . . . . . . . . . . . . . . . . 158

8.2 Subsistema de medição . . . . . . . . . . . . . . . . . . . . . . . . 159

8.2.1 Determinação da rede topográfica espacial . . . . . . . . . . . . . . 159

8.2.2 Determinação das coordenadas espaciais do ponto de monitoramento 166

8.3 Subsistema de comunicação . . . . . . . . . . . . . . . . . . . . . . 169

8.4 Subsistema de processamento e alarme . . . . . . . . . . . . . . . . . 171

9 RESULTADOS $\quad 177$

9.1 Dados de entrada . . . . . . . . . . . . . . . . . . . . . . . 177

9.2 Resultados dos métodos . . . . . . . . . . . . . . . . . . . . 183

9.3 Análises comparativas . . . . . . . . . . . . . . . . . 205

10 CONCLUSÕES E RECOMENDAÇÕES 219

10.1 Conclusões . . . . . . . . . . . . . . . . . . . . . . . . . . 219

10.2 Recomendações . . . . . . . . . . . . . . . . . . . . 224

$\begin{array}{ll}\text { Referências } & 227\end{array}$

$\begin{array}{ll}\text { Anexos } & 233\end{array}$

ANEXO A CERTIFICADO DE CALIBRAÇÃO 235

A.1 Certificado de calibração da TCA1201+ . . . . . . . . . . . . . . . . 236

A.2 Certificado de calibração da TCRP1201+R300 . . . . . . . . . . . . . 237

A.3 Certificado de calibração da TCRA1201+R400 . . . . . . . . . . . . . 238 


\section{Capítulo}

\section{MONITORAMENTO DE ESTRUTURAS}

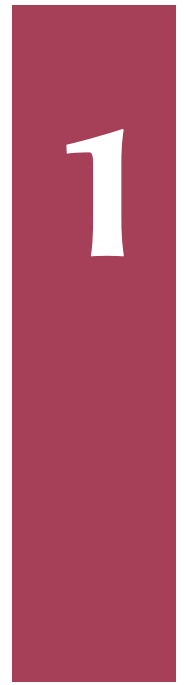

\subsection{Introdução}

Monitorar significa acompanhar o desempenho de algum evento, com o objetivo de avaliar alterações de comportamento. À ação de monitorar dá-se o nome de monitoramento ou monitoração. Assim, monitoramento é o acompanhamento, a avaliação e o controle das condições e dos fenômenos, naturais ou artificiais, com o objetivo de obter dados quantitativos e qualitativos que possibilitem maior conhecimento sobre eles, identificando, assim, possíveis riscos ou oportunidades que possam ser controlados ou aproveitados, para minimizar eventos indesejáveis. Conceitua-se, portanto, o termo monitoramento de estruturas como um processo de medição sistemática do deslocamento de objetos submetidos a ações de carga, com o objetivo de controlar o movimento de pontos desse objeto, para assegurar-se de que eles estão compatíveis com os padrões de comportamentos estruturais predefinidos. Pode-se dizer que qualquer objeto, natural ou artificial, está sujeito a variações, em sua dimensão, forma e posição, no espaço e no tempo, e esta variabilidade pode ser analisada por meio de medições de deslocamentos e de estudos sobre o comportamento dos corpos deformáveis. Dessa forma, toda estrutura física, que possa apresentar riscos, deve ser supervisionada durante toda a sua existência para permitir a detecção precoce de possíveis movimentações que podem culminar em colapso (SZOSTAK-CHRZANOWSKI; MASSIERA, 2006).

Geralmente, a instabilidade estrutural ocorre em função de ações externas que causam alterações relevantes nas tensões internas da estrutura, como, por exemplo, ação de cargas elevadas, que podem gerar tensões próximas do limite de ruptura, preconizado para a estrutura; forças externas críticas, que podem comprometer a estabilidade da estrutura; além de outras ações externas, que podem causar deformações lentas e de longo prazo na estrutura; também 
alterações no meio físico, que podem comprometer a confiabilidade do modelo matemático adotado para o dimensionamento da estrutura; e desastres naturais, que podem alterar as condições de contorno da estrutura, por meio de alterações na correlação das forças de equilíbrio do meio físico circundante (BERGMEISTER et al., 2003).

É importante salientar que devido às melhorias nas qualidades físicas dos materiais e no arrojo das construções, a engenharia passou a considerar o monitoramento não apenas como um recurso para a análise de deformações e de deslocamentos, mas também como um indicador de alterações significativas em algum parâmetro de alerta, sugerindo outras investigações sobre o comportamento da estrutura. O monitoramento passou a permitir, assim, que o engenheiro aumente a sua produtividade e a sua rentabilidade, adotando coeficientes de segurança menos restritivos (ASCE, 2010). Em princípio, segundo Cranenbroeck (2011), todo tipo de estrutura artificial e de evento natural, passível de movimentos estruturais relevantes, deve ser monitorado. Na prática, porém, devido ao alto custo do empreendimento, apenas as estruturas ou eventos que podem acarretar riscos iminentes à vida humana ou ao patrimônio são monitoradas, como, por exemplo, edifícios esbeltos, barragens, pontes e viadutos de estrutura complexa, áreas de construções instáveis, túneis, taludes de minas ou de rodovias e ferrovias, bem como encostas instáveis e outros.

O monitoramento de tais estruturas, embora seja uma área de estudo antiga para a engenharia estrutural, pode-se dizer que ainda é um objeto de estudo recente para as ciências geodésicas, mas que vem obtendo destaque neste cenário, com os avanços tecnológicos dos instrumentos topográficos e geodésicos, das técnicas de medições, dos métodos de processamento e dos recursos computacionais (EROL, 2010). A este respeito, para as ciências geodésicas, as técnicas de monitoramento estão divididas em duas categorias, que são as técnicas não geodésicas e as geodésicas.

A técnica de monitoramento é não geodésica, quando ela se baseia na implantação de sensores acoplados ao objeto estrutural, normalmente engastados na estrutura durante a fase de construção ou instalados após o término da obra, tais como, inclinômetros, piezômetros, pêndulos, extensômetros mecânicos e elétricos, termômetros, fios de prumo, sensores de fibra ótica, sensores de emissão acústica e outros. Já a técnica de monitoramento é geodésica, quando ela é realizada por meio de sistemas, métodos e instrumentos geodésicos tais como, estações totais, sensores de inclinação, níveis automáticos e digitais, escâneres a laser terrestre e receptores de sinais de satélites artificias.

A particularidade das técnicas não geodésicas é que elas, na maioria dos casos, proporcionam apenas informações de deslocamentos pontuais, localizados e sem referência, enquanto as técnicas geodésicas fornecem o estado global da estrutura e permitem verificar estatisticamente os resultados, por meio da avaliação das acurácias individuais e globais do sistema de medição, além de fornecerem versatilidade e adequabilidade para qualquer ambiente e situação de operação (CHRZANOWSKI et al., 1992). 
Com os avanços tecnológicos das últimas décadas, que potencializaram o Global Navigation Satellite System (GNSS) e que inovaram os instrumentos topográficos com servomecanismos e sensores de detecção de movimentos, o monitoramento geodésico passou a ser uma opção altamente confiável para o monitoramento de estruturas, cuja determinação das coordenadas espaciais, neste caso, pode ser realizada por meio de medições de ângulos e distâncias ou por meio do rastreamento de satélites artificiais.

No caso das técnicas baseadas na tecnologia GNSS, as medições são realizadas por intermédio de receptores e antenas GNSS, instalados sobre os pontos a serem monitorados, cuja determinação das coordenadas é realizada por meio do emprego do método de posicionamento GNSS relativo, em modo Real Time Kinematic (RTK) ou em modo pós-processado, dependendo do tipo de estrutura monitorada.

Já no caso das técnicas baseadas nas medições de ângulos e distâncias, as observações são realizadas por meio de instrumentos topográficos, denominados estações totais, mais especificamente, por meio de Estações Totais Robóticas (ETR), como pode ser visto em Palazzo et al. (2006), ou escâneres a laser terrestre, como pode ser visto em Tsakiri, Lichti e Pfeifer (2006), instalados em pilares remotos, que medem ângulos e distâncias entre o pilar e os pontos a serem monitorados individualmente ou em nuvem. A determinação das coordenadas, neste caso, é realizada por meio do emprego do método de medição polar, em que cada ponto é observado individualmente, com origem em uma única estação de monitoramento.

Existem, entretanto, algumas situações em que o uso de uma estação total robótica ou da tecnologia GNSS, individualmente, não é suficiente para realizar o monitoramento (CHRZANOWSKI et al., 1986). A solução, aqui, tem sido o uso de ambas as tecnologias, combinadas com sensores de inclinação e geotécnicos, gerando informações online e em modo contínuo sobre o comportamento da estrutura (MASCARENHAS, 2009).

Neste contexto, é importante ressaltar que a prioridade nesta tese é desenvolver estudos e propostas para um sistema de monitoramento geodésico de estruturas com o uso de várias estações totais robóticas operando simultaneamente por meio de métodos de medições multipolares para determinações espaciais de pontos individuais em deslocamento. Contrapõe-se assim aos métodos de monitoramento geodésico que utilizam somente uma estação total robótica e se baseiam no método de medição polar, os quais, evidente, são mais simples de serem aplicados, porém, não permitem nenhuma detecção de erros grosseiros ou sistemáticos e tampouco a avaliação da confiabilidade dos resultados obtidos.

Por tais razões, as pesquisas científicas sobre este tema têm-se concentrado no desenvolvimento de técnicas baseadas no uso de redes de estações de referência, em que os ângulos e as distâncias, relativas aos pontos de monitoramento, são observadas, concomitantemente, a partir de vários pilares, denominados métodos de medições multipolares. Neste caso, devido à existência de redundância de observações, as coordenadas espaciais dos pontos de monitoramento são determinadas, por meio do emprego de métodos de ajustamento de observações, que 
permitem avaliar a precisão e a confiabilidade dos resultados (EL-DIN, 2014). Trata-se, evidentemente, de um método de medição que exige maior conhecimento técnico para a configuração da rede e para o tratamento posterior dos dados, mas que, mesmo assim, vem conquistando adeptos em todos os setores relacionados ao monitoramento de estruturas.

Do ponto de vista científico, essa nova oportunidade tecnológica permite que se apliquem novos processos de medições e determinações de coordenadas espaciais no monitoramento de estruturas. Dentro deste contexto, foi desenvolvido no Laboratório de Geomática do Departamento de Engenharia de Transportes da Escola de Engenharia de São Carlos da Universidade de São Paulo (STT/EESC/USP) um sistema de monitoramento geodésico de estruturas com a utilização de variadas estações totais robóticas. O grande diferencial deste sistema é o emprego de modelagens matemáticas que consideram medições multipolares e têm como fundamentação teórica a análise numérica, especificamente no Método de Newton Generalizado, utilizado para operar na minimização dos deslocamentos em função dos parâmetros medidos e obter soluções de seus sistemas não lineares gerados, com convergência quadrática. Para estas modelagens, deu-se o nome de métodos multipolares iterativos de aproximações sucessivas para determinação de coordenadas espaciais. Tais métodos iterativos baseiam-se, a princípio, em determinar uma função que depende das observações combinadas de ao menos duas estações totais, de tal forma que essas funções tendam a zero, ou seja, a resolução de um sistema não linear que forneça uma estimativa para as coordenadas espaciais do alvo, pois, ao repetir o processo de forma iterativa, obtém-se uma melhor aproximação para as coordenadas reais do alvo e, naturalmente, ao considerar as respectivas equações de erros, realiza-se o ajustamento das incógnitas e das observações, o que torna estes métodos mais robustos, confiáveis e eficientes do que os seus predecessores.

O sistema proposto foi instalado para validação no Campus 2 da USP São Carlos. A escolha do local se deu pela necessidade de as medições precisarem ser realizadas em um ambiente aberto, com área consideravelmente extensa e com longas distâncias intervisíveis entre todos os instrumentos e os pontos de monitoramento. Considera-se que nestas condições os modelos de medição e de cálculos empregados sofrerão interferências dos efeitos atmosféricos e se tornarão realísticos do ponto de vista da aplicação prática. Desta forma, com a análise dos resultados e conclusões obtidas, foi possível verificar a qualidade de cada método empregado na determinação das coordenadas espaciais dos pontos monitorados.

\subsection{Objetivos}

Conforme descrito, a maioria dos sistemas de monitoramento geodésico de estruturas com o uso de estações totais robóticas opera com apenas uma estação monitorando os alvos em deslocamento. Neste contexto, esta tese tem como objetivo geral propor um sistema de monitoramento geodésico de estruturas integrado, que torne automática a coleta e o processamento de dados obtidos pela interseção das linhas de visadas de várias estações totais robóticas ope- 
rando simultaneamente. Os equipamentos, neste caso, são posicionados em pilares de centragem forçada, com coordenadas espaciais conhecidas e ajustadas, por meio da instalação de uma rede topográfica espacial na área de monitoramento. Acredita-se que este sistema com gestão de informação integrada, em forma de rede e com posicionamento multipolar dos pontos de monitoramento, seja uma solução mais eficiente e mais confiável para se obter maior poder de decisão, mais agilidade na detecção de eventos indesejáveis e, consequentemente, atitudes mais acertadas em todo o gerenciamento de uma auscultação estrutural. Para a concepção e a validação de um sistema deste tipo, é necessário estabelecer objetivos específicos, tais como o desenvolvimento dos subsistemas de medição, de comunicação e de processamento e alarme, como descrito ao longo desta tese.

Na elaboração do subsistema de medição, foi desenvolvido uma rede topográfica espacial, composta por métodos de medições e por modelos matemáticos, indicados para a determinação e o ajuste de coordenadas espaciais. Estabeleceu-se, assim, uma relação analítica entre todas as observáveis envolvidas no processo de cálculo de todos os pontos que compõem esta rede. Tem-se, assim, uma solução unívoca que considera todos os componentes da rede simultaneamente, diferentemente das modelagens aplicadas até então, que calculam, em separado, cada componente envolvido no processo, tratando-os independentemente.

É importante salientar que, para a finalidade de monitoramento, é necessário verificar a estabilidade das estações de medições da rede de referência para identificar e quantificar possíveis deslocamentos ocorridos na mesma. Os valores, quando detectados, devem ser introduzidos nos modelos de determinações das coordenadas espaciais dos pontos de monitoramento. Utiliza-se, neste caso, o método de medição denominado estação livre ou recessão espacial.

Para complementar o subsistema de medição, foram desenvolvidos dois modelos matemáticos para a determinação e o ajuste de coordenadas espaciais por meio de medições multipolares. O primeiro modelo considera o uso simultâneo de duas estações totais robóticas e, o segundo, o uso de três estações totais robóticas simultâneas. Ambos se baseiam em modelos matemáticos iterativos de aproximações sucessivas em função das direções e das distâncias inclinadas medidas.

Na elaboração do subsistema de comunicação, foram desenvolvidos sistemas eletrônicos para operação, gestão e transmissão integrada e, em tempo real, dos dados observados pelas estações totais robóticas, os quais são basicamente compostos por cabos de energia, de transmissão de sinais, por conversores de mídia e antenas de rádio.

Na elaboração do subsistema de processamento e alarme, foi desenvolvido um software por meio de programações na plataforma Python, em conformidade com Lutz (2013) e Cormen, Charle e Ronald (2009), que basicamente contém algoritmos e rotinas computacionais para organização, armazenamento, processamento integrado e, em tempo real, de todos os dados observados. O software permite analisar os resultados, realizar visualizações gráficas e numéricas dos vários métodos de determinação de coordenadas espaciais empregados nesta pesquisa. 
Para a devida validação do sistema de monitoramento geodésico proposto, foi desenvolvido um subsistema para simulação de deslocamentos e instabilidades estruturais, o qual é composto por um braço robótico e um sistema operacional, programado na plataforma Python, para controlar os deslocamentos e executar as tarefas de interesse, relativas aos pontos de monitoramento.

Por fim, foi realizada uma revisão detalhada da literatura científica, além da apresentação e aplicação dos métodos de medições e de modelos matemáticos disponíveis para determinação de coordenadas espaciais. Obteve-se, dessa forma, embasamento teórico e fundamentos para análises comparativas entre os métodos estudados e os que se propõem neste trabalho.

\subsection{Justificativas}

O fato de qualquer objeto, natural ou sintético, estar sujeito a variações, em sua dimensão, forma e posição, no espaço e no tempo já mostra a importância de estudos sobre o comportamento dos corpos deformáveis e pesquisas na linha de monitoramento de estruturas. Além disso, deve-se considerar que as obras da engenharia contemporânea mostram-se altamente tecnológicas e audaciosas, como é o caso de edifícios esbeltos com centenas de metros de altura, obras de arte com estruturas inovadoras, alterações significativas do meio físico, dentre outros (CHRZANOWSKI, 2009).

Tal relevância é enfatizada quando Chen (1983) afirma que, mesmo uma pequena alteração da forma do objeto ou mínimas alterações nas áreas circundantes, devem ser levadas em consideração, por mais insignificantes que pareçam, pois, podem comprometer a integridade de toda a estrutura. Podem trazer, primeiramente, riscos iminentes à vida humana e, posteriormente, ao meio ambiente e ao patrimônio, além de gerar grande impacto em todas as esferas da sociedade, o que é facilmente percebido com as grandes tragédias que abalaram o mundo recentemente, como a queda da ponte Morandi em 2018, na cidade de Gênova, no norte da Itália.

No Brasil, foram inúmeras as ocorrências de forma sistemática que ceifaram centenas de vidas, deixaram danos ambientais imensuráveis, além de prejuízos econômicos e morais. Estes eventos lastimáveis foram divulgados pela maioria dos veículos de comunicação e se tornaram de domínio público, como o desabamento do viaduto na marginal Pinheiros, em 2018, na cidade de São Paulo e os rompimentos das barragens nas cidades mineiras de Mariana e Brumadinho, em 2015 e 2019, respectivamente. Estes acontecimentos recentes repercutiram em toda a nação e expuseram para a sociedade a real necessidade de se monitorar estruturas.

Entretanto, deve-se destacar que outras estruturas podem estar em iminência de colapso e necessitam ser auscultadas. Assim, é nítido que acompanhar uma estrutura de grande porte durante toda sua vida útil é uma situação muito complexa e delicada e, devido a isso, exigem cada vez mais soluções redundantes que podem minimizar possíveis falhas e erros (ONU, 2010). 
Por fim, como citado, os principais sistemas de monitoramento geodésico de estruturas utilizam-se de apenas uma estação total robótica e empregam o método polar para determinação das coordenadas espaciais de cada alvo individualmente. Podem haver casos em que se utilizam duas ou mais estações totais robóticas, porém, sempre em grupos diferentes de alvos. Mesmo quando as medições são realizadas de forma simultânea, os métodos de cálculo empregados para a determinação das coordenadas espaciais se baseiam apenas na determinação da média das coordenadas sem o emprego de ajustamentos de observações.

Apresentar uma avaliação do emprego dos métodos conhecidos de determinação de coordenadas espaciais por meio de ajustamento de observações e propor um sistema de monitoramento geodésico de estruturas com o uso simultâneo de várias estações totais robóticas, empregando métodos de medições e determinações multipolares é o que justifica e motivou o desenvolvimento desta tese.

\subsection{Estrutura da tese}

Para organização e estruturação das informações desta tese, o documento foi dividido em 10 capítulos.

No Capítulo 1, caracteriza-se a relevância da pesquisa sobre monitoramento de estruturas, os objetivos, as justificativas e estrutura da tese.

No Capítulo 2, apresentam-se os principais conceitos relacionados aos sistemas de monitoramento geodésico.

No Capítulo 3, apresentam-se os detalhes dos instrumentos e acessórios utilizados para a realização do sistema de monitoramento geodésico proposto.

No Capítulo 4, realizam-se revisões das técnicas de medições para o monitoramento geodésico de estruturas.

No Capítulo 5, realizam-se revisões dos modelos matemáticos existentes na literatura e que são utilizados para a determinação de coordenadas espaciais.

No Capítulo 6, apresentam-se os métodos de determinações de coordenadas espaciais propostos para a rede espacial.

No Capítulo 7, apresentam-se os métodos de determinações de coordenadas espaciais propostos para pontos de monitoramento.

No Capítulo 8, apresenta-se todo o sistema de monitoramento geodésico proposto, com o uso simulatâneo de estações totais robóticas.

No Capítulo 9, apresentam-se as análises das observações de campo e os resultados obtidos.

No Capítulo 10, apresentam-se as avaliações, conclusões e recomendações para o desenvolvimentos de novas pesquisas. 
Em seguida, listam-se as referências utilizadas no desenvolvimento da pesquisa. Por fim, apresentam-se outros documentos no Anexo A. 


\section{Capítulo}

\section{MONITORAMENTO GEODÉSICO DE ESTRUTURAS}

\subsection{Sistemas de monitoramento geodésicos de estruturas}

O monitoramento geodésico, devido à sua amplitude de aplicações, técnicas de medições, métodos de tratamento dos dados, instrumentos de medição e equipamentos acessórios, constituise em um sistema de gestão de dados composto de vários componentes que, em conjunto, permitem realizar o monitoramento de uma estrutura. O objetivo final da aplicação desse sistema é a determinação periódica das coordenadas espaciais de pontos específicos de uma estrutura para, a partir delas, calcular as deformações ou os deslocamentos da mesma (ARMY, 1994). Para alcançar esse objetivo, um sistema de monitoramento deve ser composto de três subsistemas, conforme indicado a seguir:

- Subsistema de medição;

- Subsistema de comunicação;

- Subsistema de processamento de dados e de alarmes.

Discute-se na sequência os componentes de cada subsistema. Na Figura 2.1, estes subsistemas são apresentados na sequência lógica de implantação do monitoramento geodésico de uma barragem. 


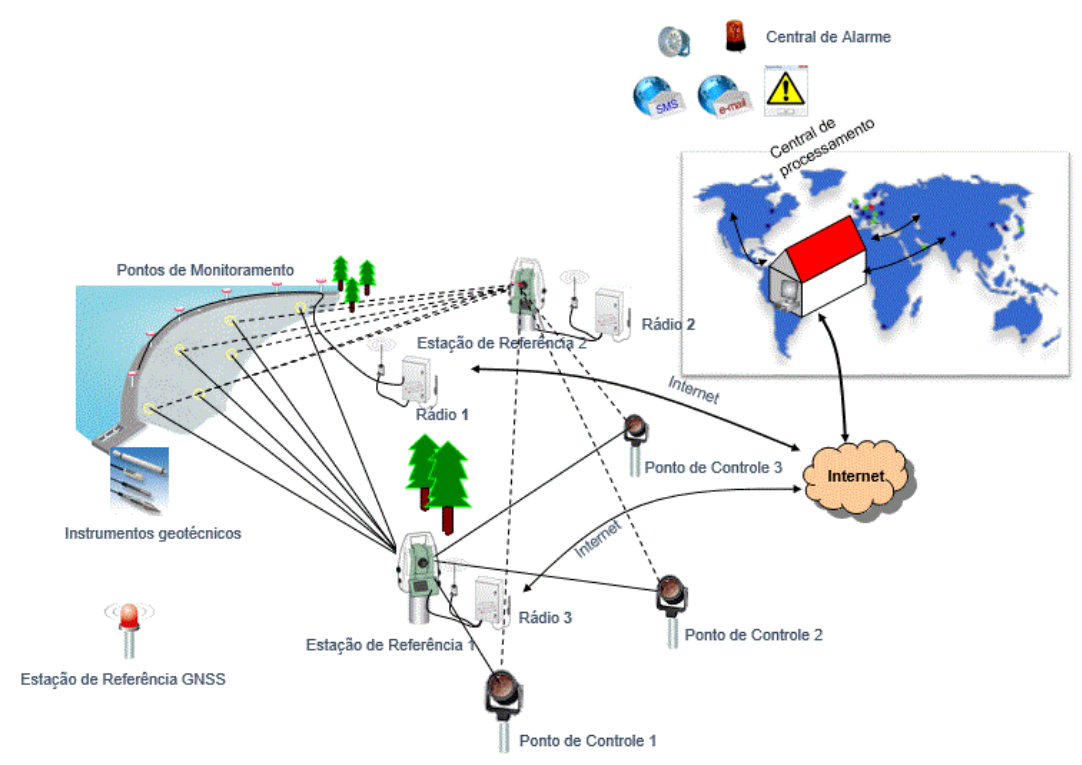

Figura 2.1: Componentes de um sistema de monitoramento geodésico.

\subsubsection{Subsistema de medição}

Dependendo do tipo e das condições físicas da estrutura, o monitoramento pode ser de curto ou de longo prazo, sendo mais comuns os de longo prazo. Em ambos os casos, a metodologia de medição baseia-se no estabelecimento de uma rede de pontos de apoio topográficos interconectados por medições de direções, ângulos e/ou distâncias, denominados pontos de referência ou estações de referência, a partir dos quais são determinadas as coordenadas espaciais de pontos selecionados sobre a superfície do objeto em análise, denominados pontos de monitoramento. Os pontos de referência também precisam ser monitorados de tempos em tempos, para verificação da sua estabilidade. O monitoramento desta etapa é feito a partir de outros pontos de referência, denominados pontos de controle, que devem estar localizados fora da área de ação da estrutura monitorada. O conjunto desses pontos, com seus monumentos e processos de medição, forma o subsistema de medição.

\subsubsection{Subsistema de comunicação}

Após o processo de medição, os valores observados são armazenados nos instrumentos de medição, em computadores conectados a eles ou são enviados online para uma central de processamento. O conjunto de instrumentos e procedimentos de transferência de dados forma o subsistema de comunicação.

\subsubsection{Subsistema de processamento}

A central de processamento além de armazenar os dados, processá-los e analisá-los, possui também a incumbência de controlar as atividades de medições dos instrumentos remotos e de emitir os sinais de alarmes. Trata-se, portanto, do subsistema CORE do monitoramento, que 
gerencia todo o processo de medição e efetua ações interativamente com todos os elementos ativos do monitoramento.

Assim, por se tratar de um sistema complexo, a implantação de um monitoramento geodésico deve ser precedido de um projeto de monitoramento bem detalhado, para garantir a consecução das operações.

\subsection{Projetos de monitoramento geodésico de estruturas}

Como qualquer projeto de engenharia, o de monitoramento geodésico é composto de várias etapas, conforme se descreve a seguir:

- Estudo do objeto de monitoramento para a definição das diretrizes do projeto, como deformações ou deslocamentos esperados e níveis de precisão;

- Plano de medição;

- Plano de Instrumentação;

- Definição do sistema de comunicação para gestão das medições e transferência de dados;

- Definição da periodicidade do monitoramento;

- Plano de processamento e avaliação dos resultados das medições.

\subsubsection{Estudo do objeto de monitoramento}

Os objetos de monitoramento podem ser diversos. Geralmente, estruturas naturais ou artificiais instáveis, monitoradas durante todo o seu tempo de existência, como no caso de uma barragem, por exemplo, ou somente durante um período predeterminado, como no caso da construção de um edifício esbelto. O plano de monitoramento, segundo (BESHR; KALOOP, 2013), deve ser traçado ainda na fase de projeto da estrutura e deve garantir que todos os pontos críticos possam ser observados com redundâncias e precisões predeterminadas. Os pontos de referência são, geralmente, implantados durante a construção da obra e os pontos de monitoramento são implantados na fase final, o que depende do tipo de monitoramento. No caso de uma obra de construção civil, a localização dos pontos de monitoramento é definida pelo projetista, em acordo com o construtor e o engenheiro responsável pelo monitoramento (ALMEIDA; ALEXANDINO; FERNANDES, 2008). No caso de uma estrutura natural, a localização dos pontos é definida pelos engenheiros do órgão responsável pela segurança civil, em acordo com o engenheiro responsável pelo monitoramento. Em ambos os casos, a determinação do nível da precisão exigida e da precisão possível de ser alcançada devem ser estabelecidas em consenso entre todos os profissionais envolvidos no projeto (CHRZANOWSKI et al., 1981). 


\subsubsection{Plano de medição}

O plano de medição é realizado em função do tipo de estrutura a ser monitorada, das precisões e dos resultados esperados. Por esta razão, ele pode diferir, consideravelmente, entre diferentes projetos. Mesmo assim, existem alguns parâmetros básicos que devem ser considerados para todos os projetos, conforme se indica a seguir:

- Análise do comportamento físico da estrutura;

- Requerimentos de precisão do posicionamento dos pontos de monitoramento;

- Requerimentos de precisão das medições;

- Quantidade e tipos de medições;

- Seleção da quantidade e dos tipos de instrumentos de medição;

- Procedimentos de medições de campo e de coleta de dados.

O comportamento físico da estrutura é uma característica própria de cada estrutura e do carregamento a que ela será submetida. As considerações sobre este parâmetro do monitoramento é de responsabilidade do engenheiro projetista ou do órgão responsável pela segurança civil (CHEN; CHRZANOWSKI, 1986).

Em função das características relacionadas ao comportamento físico da estrutura, é que se definem os requerimentos de precisão do posicionamento dos pontos de monitoramento e, em função deles, os requerimentos de precisão das medições. Em geral, adota-se o fluxograma de operações e requerimentos indicados na Figura 2.2.

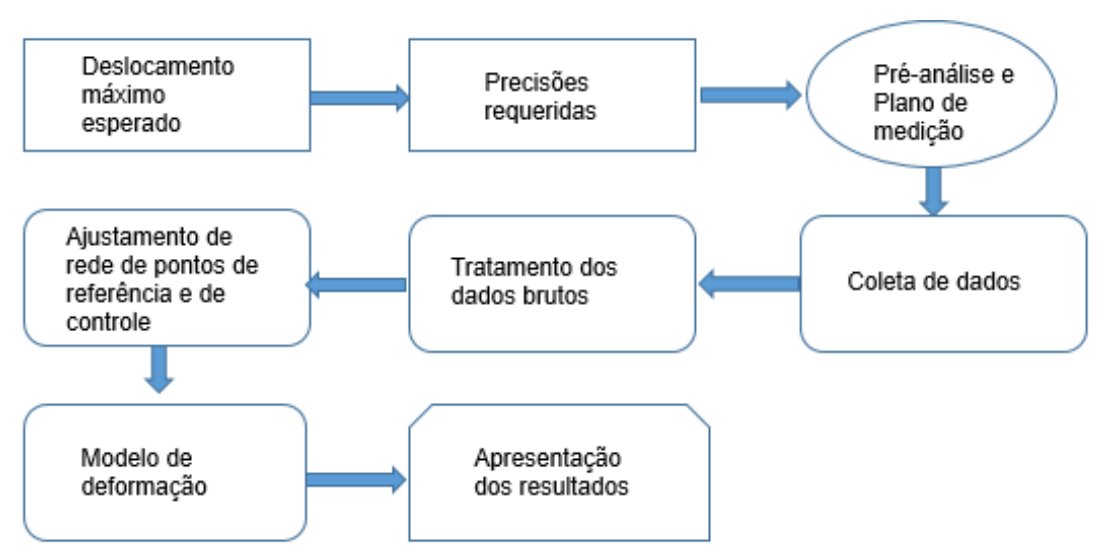

Figura 2.2: Fluxograma de operações de monitoramento.

Em relação aos níveis esperados de deslocamento e das precisões dos posicionamentos dos pontos de monitoramento, apresenta-se a seguir os requisitos de precisão para algumas estruturas padrões indicados em ARMY (2002), como pode ser visto na Tabela 2.1. 
Tabela 2.1: Requisitos de precisões para obras de engenharia civil (95\% RMS)

\begin{tabular}{lll}
\hline Tipo de Estrutura & & Precisão \\
\hline \multirow{2}{*}{ Estruturas de concreto } & Movimento de longo termo & \pm 5 a $10 \mathrm{~mm}$ \\
\cline { 2 - 3 } & Deflexões de curto prazo & $\pm 0,2 \mathrm{~mm}$ \\
\cline { 2 - 3 } & Estabilidade vertical & $\pm 2 \mathrm{~mm}$ \\
\hline \multirow{2}{*}{ Barragens } & Estabilidade da crista e do talude & \pm 20 a $30 \mathrm{~mm}$ \\
\cline { 2 - 3 } & Alinhamento da crista & \pm 20 a $30 \mathrm{~mm}$ \\
\cline { 2 - 3 } & Recalques & $\pm 10 \mathrm{~mm}$ \\
\hline Estruturas diversas & Instabilidades & \pm 5 a $10 \mathrm{~mm}$ \\
\hline
\end{tabular}

A precisão possível de ser alcançada no processo de medição dependerá do tipo de instrumentos utilizados, dos procedimentos de medição e dos métodos de determinação de coordenadas. Os instrumentos, geralmente, são escolhidos em função da resolução e da precisão nominal indicada pelo fabricante. Mesmo assim, depois de adquiridos, eles devem ser verificados e ajustados em laboratórios especializados, antes da primeira campanha de medição e, periodicamente, nas campanhas subsequentes (ALBA; PONCORONI; SCAIONI, 2008).

Em relação às medições de campo e de coleta de dados, os procedimentos a serem seguidos dependerão da periodicidade das medições e dos modelos matemáticos empregados para o processamento e a análise dos dados.

\subsubsection{Plano de instrumentação}

O plano de instrumentação consiste na determinação da rede de pontos de monitoramento, da rede de pontos de referência e da rede de pontos de controle, além da determinação dos instrumentos e acessórios a serem utilizados nas medições. Em geral, o plano deve conter especificações e procedimentos relacionados aos itens indicados a seguir:

- Tipos de instrumentos, de acessórios e de materiais diversos a serem utilizados em todas as fases do monitoramento;

- Tipos e localização dos monumentos, como pilares de medição, de monitoramento e de apoio, com a indicação da função de cada um deles;

- Procedimentos de instalação, de proteção e de manutenção dos monumentos.

Os instrumentos e os acessórios a serem utilizados no monitoramento dependerão do tipo de monitoramento. Em geral, conforme já citado, para o monitoramento geodésico, são utilizados estações totais robóticas, níveis digitais, sensores de inclinação, receptores GNSS e escâneres 
terrestres, com os seus devidos acessórios. Tais instrumentos podem ser usados individualmente ou em conjunto, dependendo do tipo de movimento da estrutura que se deseja analisar e da geometria planejada para o monitoramento.

Os monumentos utilizados para o monitoramento geodésico de estruturas consistem, basicamente, de pilares de medição cravados em rochas ou pinos de monitoramento fixados na estrutura, tanto para a operação com estações totais robóticas, quanto para o uso de receptores e antenas GNSS. Os monumentos para as estações de referência, em geral, são pilares em concreto ou em metal, localizados em áreas remotas em relação à estrutura. Eles devem ser instalados em pontos estáveis do terreno, preferencialmente cravados em rochas e devem garantir estabilidade horizontal e vertical, em relação aos pontos de controle. A instalação dos instrumentos sobre esses pilares é sempre realizada com dispositivos de centragem forçada. Porém, a instalação de pilares de concreto e de pilares metálicos nem sempre é possível de ser realizada e, consequentemente, o monumento de instalação, muitas vezes, precisa ser improvisado. Também em instalações remotas, em que fontes de energia podem ser um problema, tem-se empregado montagens com painéis solares.

No caso dos monumentos para os pontos de monitoramento, são utilizados blocos ou pilares de concreto, hastes ou pinos metálicos de medição fixados na estrutura monitorada. Eles devem, evidentemente, ser instalados nos pontos definidos como de máxima deformação da estrutura ou suspeitos de instabilidade. A distribuição dos pontos deve ser feita de acordo com o padrão de risco indicado para os diferentes setores da estrutura. Novos pontos podem ser adicionados sempre que um setor da estrutura mostrar comportamento suspeito. A este respeito, existem várias opções de instalação, muitas delas, também improvisadas, em função das especificidades da estrutura ou da obra (BESHR; KALOOP, 2013).

Nos monitoramentos com estações totais robóticas, os instrumentos devem estar sempre protegidos das intempéries, principalmente nos casos de monitoramentos contínuos. Nestas situações, geralmente, os instrumentos são instalados dentro de um abrigo envidraçado, cujo material pode ser de vidro laminado e deve-se evitar envidraçamento duplo. A estação total robótica deve ser instalada o mais próximo possível do vidro e, no caso de medições de distâncias com instrumentos com capacidade para o reconhecimento automático de prismas, a máxima eficiência nas medições ocorre quando o plano do vidro está inclinado com um ângulo de aproximadamente $20^{\circ}$ em relação à linha de visada, para medições com infravermelho e, aproximadamente $5^{\circ}$, para medições com raio laser. Além disso, instalações com ar condicionado são sempre uma garantia de maior estabilidade dos componentes internos dos instrumentos de medição. As linhas de visadas com estações totais robóticas devem estar sempre alinhadas com os prismas refletores, principalmente para as medições de distâncias longas, maiores do que $500 \mathrm{~m}$. Caso o ponto de monitoramento seja visado por mais de uma estação total robótica, pode-se optar pelo uso de prismas do tipo $360^{\circ}$. 


\subsubsection{Plano de comunicação, de gestão e de transferência de dados}

O sistema de comunicação a ser usado em um monitoramento geodésico depende do tipo de monitoramento, da periodicidade das medições, das instalações disponíveis e das distâncias de comunicação. A este respeito, existe uma série de opções disponíveis no mercado, dentre os quais se destacam:

- Cabos;

- Serial (RS232);

- Lemo;

- Universal Serial Bus (USB).

- Rádios;

- Fibra ótica;

- LAN/DSL;

- WLAN;

- GSM/GPRS;

- Comunicação via satélite artificial.

De acordo com ARMY (2002), como mostra a Tabela 2.2, existem diferentes tipos de comunicação a serem usados em função do objeto de monitoramento.

Tabela 2.2: Tipos de comunicação em função do objeto de monitoramento.

\begin{tabular}{cccc}
\hline Objeto & Característica & Distância [m] & Comunicação \\
\hline Mina a céu aberto & Áreas remotas & 1.000 a 3.000 & Rádio \\
Construções civis & Área obstruída & 300 a 2.500 & WLAN ou rádio \\
Taludes & Sem energia e intervisibilidade & 200 a 5.000 & GSM/GPRS \\
Barragens & Inferência eletromagnética & 200 a 1.500 & Cabo serial, fibra ótica \\
Sísmico & Áreas de grandes superfícies & 2.000 a 100.000 & GSM ou satélite \\
Vulcão & Áreas extensas e remotas & 1.000 a 10.000 & Satélite \\
\hline
\end{tabular}

O perfeito funcionamento do sistema de comunicação é um fator extremamente importante a ser considerado no sistema de monitoramento. Geralmente, os pontos de referência e de monitoramento se encontram distantes do centro de processamento e são de difícil acesso. Por esta razão, todo sistema de monitoramento busca meios para acessá-los remotamente. Dessa forma, toda coleta de dados e comando dos instrumentos de medição são feitos por intermédio de um sistema de comunicação remoto. O projeto de monitoramento deve, portanto, prever 
energia e facilidade de comunicação em todos os pontos de referência e nos pontos de monitoramento. A Figura 2.3 mostra um exemplo de instalação para estações totais robóticas, com seus sistemas de comunicação.

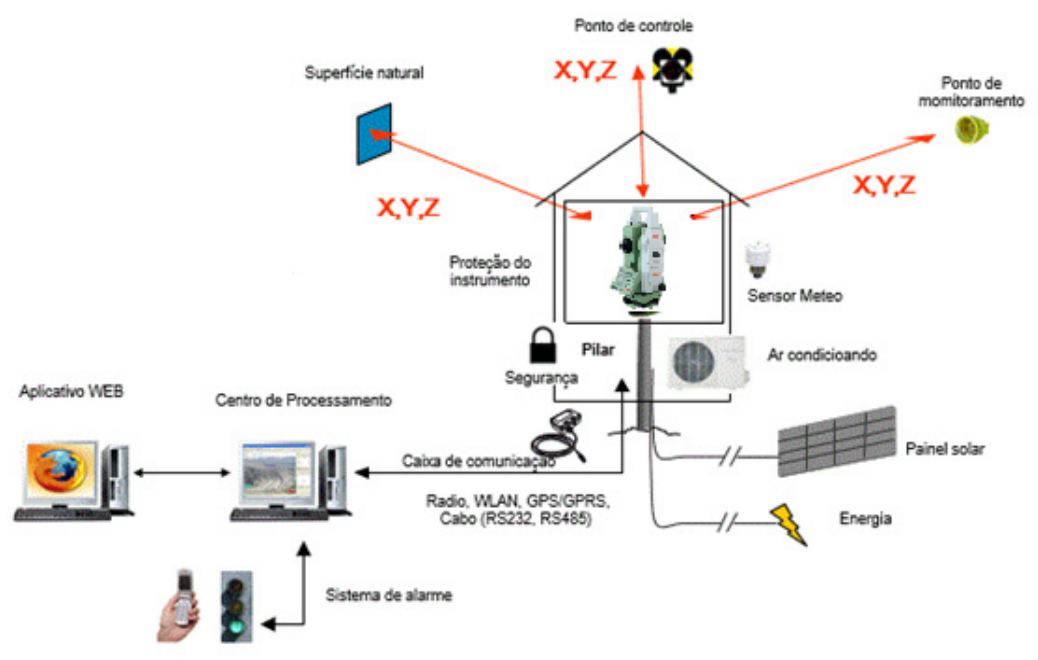

Figura 2.3: Instalação de monitoramento contínuo, com o uso de estações totais robóticas.

\subsubsection{Plano de periodicidade e monitoramento}

A periodicidade do monitoramento é um parâmetro que depende do tipo e das condições da estrutura a ser monitorada. Fatores de riscos, tais como a idade da estrutura, a classificação de risco, as regulamentações de segurança e a probabilidade de sinistro, são os indicadores para a definição da periodicidade (OGUNDARE, 1990). Existem situações em que o monitoramento em intervalos de tempo longos é aceitável, uma ou duas vezes por ano, por exemplo. Nesses casos, os instrumentos de medição são instalados apenas durante a campanha de monitoramento e as medições são realizadas, geralmente, com estações totais robóticas, níveis digitais ou escâneres terrestres. Os dados são coletados e armazenados no próprio instrumento ou em um computador de bordo, para posterior processamento no escritório. Existem situações em que o monitoramento deve ser contínuo, ou seja, todos os instrumentos e acessórios ficam instalados permanentemente e as medições são realizadas em intervalos de tempo predefinidos, que podem variar de minutos a horas, ou mesmo, dias. As medições, nesses casos, são realizadas com estações totais robóticas ou receptores GNSS e os dados coletados são transferidos do local de medição para uma estação de processamento remota em tempo real, via sistemas de comunicação (BIRD, 2009).

Nos casos de monitoramento contínuo, em geral, a área de monitoramento é setorizada em níveis de riscos. Segundo Beshr (2015), deve-se definir as zonas críticas, que devem ser monitoradas com maior regularidade e as zonas de menor risco, que podem ser monitoradas com menor regularidade. 


\subsubsection{Plano de processamento, avaliação dos dados e alarme}

Os dados coletados em campo, durante a fase de medição, são denominados dados brutos. Eles consistem de informações geradas pelos instrumentos de medição que, em geral, precisam ser tratadas adequadamente antes de serem processadas. O tipo de tratamento a ser efetuado no dado medido depende do tipo de instrumento. Em geral, o processamento dos dados consiste nas seguintes operações:

- Descarga dos dados;

- Verificação da consistência dos dados submetidos;

- Cópia de segurança (backup);

- Organização em um banco de dados consistente;

- Tabulação ou codificação para entrada em programas de computadores;

- Cálculo das médias das observações, se for o caso;

- Reduções geométricas das observações, se for o caso;

- Pré-processamento para avaliação a priori;

- Processamento em programas aplicativos de computador;

- Avaliação dos dados;

- Geração de relatórios.

Evidentemente, ao considerar o elevado nível de precisão esperado para um projeto de monitoramento, deve-se sempre utilizar instrumentos geodésicos da mais alta qualidade e empregar metodologias de medição e de processamento dos dados, que garantam a qualidade das medições e dos resultados obtidos e também a consistência matemática para a avaliação estatística dos resultados. Em um monitoramento geodésico, as grandezas a serem medidas são, primordialmente, ângulos e distâncias. A partir das observações dessas grandezas, são determinadas as coordenadas espaciais $(X, Y, Z)$ dos pontos de monitoramento, em conjunto ou em separado, em coordenadas planimétricas $(X, Y)$ e coordenada altimétrica $Z$. O deslocamento ou o movimento do ponto de monitoramento é dado pela análise da variação dessas coordenadas. Para tanto, define-se um instante $t_{0}$ de referência, com coordenadas $\left(X_{0}, Y_{0}, Z_{0}\right)$, as quais são comparadas com os valores das coordenadas determinadas nas campanhas de medições posteriores. As diferenças entre as componentes em $(X, Y, Z)$, nas diferentes campanhas, definem as magnitudes dos vetores espaciais, os quais, coletivamente, descrevem o comportamento da estrutura em um dado intervalo de tempo. Como ressaltam Lienhart, Ehrhart e Grick (2017), se 
algum valor de deslocamento e/ou sua velocidade, durante tempos distintos, forem superiores a valores predefinidos, considera-se que o ponto analisado está se movimentando.

Os dados processados indicarão o comportamento da estrutura e sempre que se detectar uma ocorrência inesperada, dispara-se um alarme, que pode ser visual, sonoro e por intermédio de mensagens. As diferenças de coordenadas e a velocidade do deslocamento são os dois parâmetros usados para determinar a ocorrência de comportamento suspeito. Atenção especial deve ser dada à detecção de erros grosseiros e à modelagem atmosférica para a adequada correção da refração atmosférica e da propagação do raio eletromagnético, no caso de medições de distâncias eletrônicas (BESHR; ELNAGA, 2012).

Os deslocamentos podem ser avaliados em relação à primeira campanha, de tempo $t_{0}$, ou em relação a qualquer outra campanha considerada relevante. Para o deslocamento horizontal, geralmente são criados perfis de deslocamentos prioritários, que representam a direção crítica do movimento. Os deslocamentos verticais estão em função da variação da coordenada $Z$.

Conforme exposto no início deste capítulo, o monitoramento geodésico de uma estrutura constitui-se em um sistema de gestão de dados. Dessa forma, um fator imprescindível é que seja criado um banco de dados com as informações das medições e dos resultados, ambos obtidos durante a história do monitoramento. Tais dados darão também subsídios para novos monitoramentos e permitirão análises temporais do comportamento da estrutura. Outro fator relevante a ser considerado é a qualidade das instalações, dos instrumentos e dos procedimentos de medição. Monitorar é um trabalho de altíssima precisão (WILKINS et al., 2003). 


\section{Capítulo}

\section{INSTRUMENTOS UTILIZADOS NO MONITORAMENTO GEODÉSICO DE ESTRUTURAS}

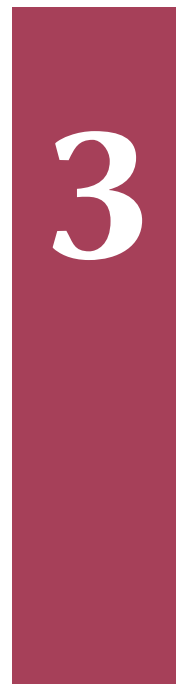

O tipo de instrumento a ser utilizado no monitoramento geodésico depende da estrutura a ser monitorada, do tipo de dados e da precisão que se deseja obter com o monitoramento, bem como do elemento do sistema de monitoramento que se deseja medir (ASCE, 2000). Em geral, são utilizados os seguintes instrumentos:

- Estações totais robóticas;

- Níveis digitais;

- Sensores de inclinação;

- Receptores GNSS;

- Escâneres a laser terrestre.

As estações totais robóticas e os níveis digitais são utilizados tanto para o levantamento das redes de pontos de apoio e de referência quanto para a medição dos pontos de monitoramento (BESHR; ELNAGA, 2012). Os receptores GNSS são utilizados primordialmente na medição dos pontos de monitoramento, embora alguns estudos indiquem também o seu uso como ponto de referência para casos específicos de monitoramento combinado de estações totais robóticas e de receptores GNSS. Já os escâneres a laser são utilizados somente na medição dos pontos de monitoramento (SUI et al., 2008). Por fim, os sensores de inclinação, juntos ao corpo estrutural, medem possíveis movimentações da mesma (YIGIT; INAL; YETKIN, 2008). Porém, nesta tese, serão descritos apenas os detalhes técnicos mais importantes das estações totais robóticas e seus acessórios, que foram os instrumentos utilizados na pesquisa. 


\subsection{Instrumentos utilizados no subsistema de medição}

Para a elaboração do sistema de monitoramento geodésico proposto, foram utilizados os seguintes instrumentos, acessórios, materiais e softwares.

\subsubsection{Estação total robótica}

Uma estação total é um instrumento topográfico que permite medir ângulos, distâncias e armazenar os valores medidos na memória interna do instrumento. Elas podem ser classificadas, atualmente, em estações totais manuais e estações totais robóticas. Uma estação total é considerada manual quando todas as operações de medição são efetuadas pelo operador. Uma estação total é considerada robótica quando possui servomecanismos, que permitem que o instrumento e a luneta girem, automaticamente, em torno dos seus eixos. Em geral, elas possuem também capacidade para reconhecimento e seguimento automático de prismas, o que as tornam adequadas para o monitoramento de estruturas (LUTES, 2002). Por serem robóticas, elas podem ser manipuladas remotamente e, por serem capazes de reconhecer um prisma, elas permitem o monitoramento automático por meio de sequências de medições definidas pelo operador.

Para o reconhecimento automático de prismas, as estações totais robóticas possuem uma câmera Charge-Coupled Device (CCD) e um sistema de autofoco, montados em conjunto com o distanciômetro e inseridos no interior da luneta do instrumento. Existem duas técnicas para o reconhecimento automático de prismas em uma estação total robótica:

- Rastreio e retorno do sinal, que se baseia na qualidade do sinal emitido e recebido de volta pela estação total robótica;

- Correlação de imagem, que se baseia na aquisição e no tratamento da imagem recebida pelo instrumento, por meio da qual o prisma é detectado.

O alcance para a medição da distância, em ambos os casos, depende do tipo de energia eletromagnética usada no processo de medição. Dessa forma, quando se utilizam instrumentos baseados em ondas eletromagnéticas do espectro infravermelho, o alcance pode chegar a $2 \mathrm{~km}$, com um prisma. Porém, quando se usam instrumentos baseados em ondas eletromagnéticas do espectro laser, o alcance pode chegar a $3,5 \mathrm{~km}$, com um prisma.

Os servomecanismos estão inseridos no interior do instrumento, juntamente com os demais componentes eletrônicos que comandam as funções da estação total robótica. Dentre os componentes de uma estação total robótica, os círculos graduados e o distanciômetro eletrônico são os elementos fundamentais do instrumento. Eles estão protegidos no interior da alidade e localizam-se, relativamente aos eixos do instrumento, de forma a permitirem observações de direções horizontais, de ângulos verticais e de medição de distâncias. 
No caso do círculo horizontal, que está localizado na parte inferior da alidade, o seu centro deve coincidir com o eixo vertical do instrumento. Já, no caso do círculo vertical, que está localizado no montante vertical da alidade, o seu centro deve coincidir com o eixo secundário do instrumento. Isso significa dizer que quando o instrumento está nivelado, o círculo horizontal está paralelo ao plano horizontal, que passa pelo centro do instrumento, e o círculo vertical está paralelo ao plano vertical, perpendicular ao horizontal.

Além dos círculos graduados e do distanciômetro eletrônico, outro componente importante a ser considerado em uma estação total robótica, para monitoramento geodésico, é o compensador eletrônico. Ele é instalado no interior do corpo do instrumento e tem a função de indicar o nível de horizontalidade do mesmo. O compensador eletrônico consiste de uma linha de padrões gravada sobre um prisma de vidro que, ao ser iluminada, é refletida duas vezes pela superfície horizontal de um líquido refletor. Cada imagem refletida da linha de padrões é lida por um arranjo linear de câmeras CCD, por meio da qual se determinam, matematicamente, as duas componentes de inclinação da estação total robótica. Os valores determinados são usados, em seguida, para o nivelamento do instrumento e para as correções de todas as medições angulares, realizadas com a estação total robótica. Geralmente, um compensador deste tipo tem um intervalo de operação da ordem de 4" de arco e precisão máxima de compensação igual a 0,5 " de arco.

As características técnicas básicas das estações totais robóticas, geralmente utilizadas em monitoramento geodésico de estruturas, estão indicadas na Tabela 3.1.

Tabela 3.1: Características técnicas básicas de estações totais robóticas para monitoramento.

\begin{tabular}{crll}
\hline Características & \multicolumn{3}{c}{ Valores } \\
\hline Precisão angular & $0,5 ”$ & e & $1,0 ”$ \\
Precisão linear & $1 \mathrm{~mm}+1 \mathrm{ppm}$ & e & $2 \mathrm{~mm}+2 \mathrm{ppm}$ \\
Alcance & $2,0 \mathrm{~km}$ & e & $3,5 \mathrm{~km}$ \\
Tempo de medição & $2,4 \mathrm{~s}$ & e & $5,0 \mathrm{~s}$ \\
\hline
\end{tabular}

Para poder alcançar os altos níveis de precisões exigidos em um monitoramento geodésico, a estação total robótica e todos os seus acessórios devem possuir alto grau de qualidade e, para tanto, devem ser manipuladas, armazenadas e transportadas com extremo cuidado. Além disso, ela deve ser calibrada, periodicamente, para garantir que os seus erros instrumentais estejam dentro dos padrões aceitáveis, para o seu uso em monitoramentos geodésicos (AFENI; CAWOOD, 2013). Deve-se ressaltar, também, que as tolerâncias de fabricação de uma estação total robótica estão no limite das possibilidades da construção mecânica. Existem, entretanto, alguns erros residuais inevitáveis de ajustagem do instrumento, que permanecem após a construção e que devem ser corrigidos, sempre que detectados.

Mas deve-se, segundo Chrzanowski e Secord (2000), considerar que todos os aparelhos geodésicos apresentam erros instrumentais, principalmente, devido às restrições impostas ao 
processo de fabricação dos mesmos. Os erros instrumentais de uma estação total robótica devem ser conhecidos para que se possa estabelecer a qualidade dos trabalhos executados e, no presente caso, estabelecer a qualidade das coordenadas obtidas por meio dos métodos de determinação de coordenadas espaciais. Em todos os trabalhos geodésicos os erros são propagados de acordo com leis específicas, matematicamente definidas, em função dos elementos envolvidos no processo de cálculo.

Dessa forma, os principais erros que ocorrem nos instrumentos devem ser analisados, bem como a influência exercida sobre as medições angulares e lineares, usadas na determinação de coordenadas espaciais.

Nos casos em que os eixos do instrumento apresentam defeitos de geometria, há alterações nos valores das observações angulares. Tais erros de eixo são apresentados abaixo:

- Erro de basculamento da luneta - ocorre quando o eixo secundário não forma perpendicular ao eixo principal;

- Erro de colimação horizontal - ocorre quando o eixo de visada não forma perpendicular ao eixo secundário;

- Erro de verticalidade do eixo principal - ocorre quando há erro de nivelamento e calagem com defeito.

É importante notar que os erros do eixo de basculamento e de colimação são eliminados realizando as medições nas duas posições da luneta e calculando-se a média das medidas. $\mathrm{O}$ recomendável é a execução de séries de medições com a luneta nas posições direta (Face I) e inversa (Face II). Por uma série compreende-se uma medida de um mesmo ângulo $n$ vezes, cada uma numa posição da luneta. Uma série inclui, portanto, $n$ visadas para cada alvo e $n$ leituras do círculo horizontal e/ou vertical em cada direção. Já o erro de verticalidade do eixo principal depende da precisão do compensador eletrônico do instrumento. Deve-se notar que esse tipo de erro não é eliminado mesmo quando se realiza medições nas duas posições direcionais da luneta. Para que uma estação total realize as medições com as precisões indicadas pelo fabricante, os três eixos do equipamento devem satisfazer as seguintes condições:

- O eixo secundário deve ser perpendicular ao eixo principal;

- O eixo de visada deve ser perpendiculara ao eixo secundário;

- O eixo principal deve estar vertical após a calagem.

Em geral, estas condições não são completamente satisfeitas. As medidas angulares são afetadas por erros de observação e instrumental, que podem ocasionar os denominados erros de 
eixos da estação total robótica, cujos efeitos nas medições angulares não podem ser desprezados. Além disso, os três eixos devem ser concorrentes em um mesmo ponto, que é o centro óptico do aparelho.

Para a medição angular, os círculos graduados devem estar perfeitamente centralizados sobre os eixos correspondentes. O centro do círculo horizontal deve coincidir com o centro do eixo principal e o centro do círculo vertical deve coincidir com o centro do eixo secundário. Se esse não for o caso, têm-se os erros de excentricidade dos círculos, apresentados abaixo:

- Erro de excentricidade do círculo - ocorre quando há erro de centragem do círculo horizontal sobre o eixo principal e erro de centragem do círculo vertical sobre o eixo secundário;

- Erro de índice do círculo vertical - ocorre quando há erro de colimação do círculo vertical em relação à linha de referência adotada;

- Erro de graduação dos círculos - ocorre quando os erros são relativos a defeitos de graduação sobre as medições angulares.

Nos equipamentos com capacidade de reconhecimento automático de prismas Automatic Target Recognition (ATR) ou com capacidade de aquisição de imagens, pode ocorrer a situação em que o alinhamento do centro da câmera CCD não coincida com a linha de visada da luneta. Este tipo de erro é particularmente importante, para os casos em que se mesclam medições manuais com medições automáticas. Para corrigi-lo, recomenda-se seguir as instruções dos fabricantes, geralmente descritas no manual do usuário do instrumento (LEICA, 2009).

Outro erro que deve ser observado durante as medições de campo é aquele que ocorre devido à diferença de temperatura entre o instrumento e o ambiente em torno dele, que pode afetar o funcionamento do compensador. Ele pode ser parcialmente corrigido, medindo-se nas duas posições da luneta. Mesmo assim, recomenda-se deixar o instrumento ajustar-se ao meio ambiente, antes de se iniciar as observações angulares. Para a maioria dos instrumentos, o tempo de ajuste à temperatura ambiente é de aproximadamente 2 minutos $/{ }^{\circ} \mathrm{C}$ de diferença entre a temperatura do instrumento e a do meio ambiente.

Os erros instrumentais são determinados e minimizados durante o processo de fabricação. Mesmo assim, eles podem sofrer variações devido a choques, alterações de temperaturas e outros. Por tais razões, recomenda-se calibrar o instrumento regularmente ou sempre que usálo pela primeira vez, após longos períodos sem uso, após mudanças bruscas de temperatura e após uso intensivo ou longas distâncias de transporte.

É importante atentar para o fato de que a maioria das estações totais robóticas, depois de calibrada, possui capacidade para considerar automaticamente os efeitos dos erros instrumentais nas observações angulares. Mesmo assim, para garantir a qualidade das observações nos tra- 
balhos de monitoramento geodésico, recomenda-se realizar sempre as observações angulares nas duas faces da luneta, direta e inversa.

Nesta pesquisa foram utilizadas três estações totais robóticas, como mostra a Figura 3.1. Os detalhes técnicos de cada instrumento estão indicados a seguir:

- TCA1201+

Estação Total Robótica de marca LEICA Geosystems, da série profissional TPS1200+, modelo Total Station Positioning System (TCA), tipo 1201+, que possui precisão angular de 1", precisão linear de $2 \mathrm{~mm}+2 \mathrm{ppm}$, dispositivos de automação e servomotores (LEICA, 2007). Vide Figura 3.1a.

- $\mathrm{TCRP} 1201+\mathrm{R} 300$

Estação Total Robótica de marca LEICA Geosystems, da série profissional TPS1200+, modelo Theomat Coaxial Automated Reflectorless Power Search Total Station (TCRP), tipo $1201+\mathrm{R} 300$, que possui precisão angular de 1”, precisão linear de $2 \mathrm{~mm}+2 \mathrm{ppm}$, dispositivos de medições sem alvos refletores, dispositivos de automação e servomotores (LEICA, 2007). Vide Figura 3.1b.

\section{- TCRA1201+R400}

Estação Total Robótica de marca LEICA Geosystems, da série profissional TPS1200+, modelo Theomat Coaxial Automated Reflectorless Total Station (TCRA), tipo 1201+R400, que possui precisão angular de 1", precisão linear de $2 \mathrm{~mm}+2 \mathrm{ppm}$, dispositivos de medições sem alvos refletores, dispositivos de automação e servomotores (LEICA, 2007). Vide Figura 3.1c.

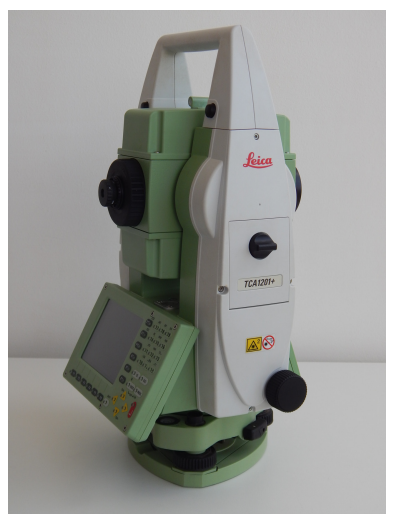

(a) TCA1201+.

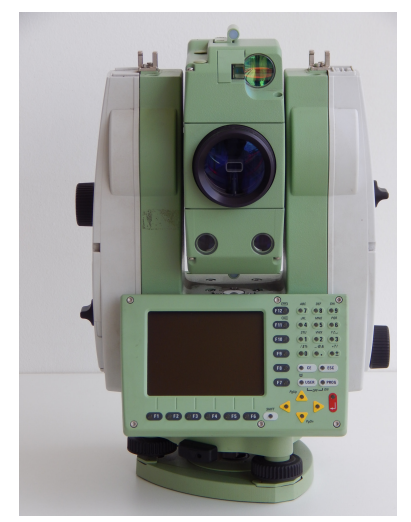

(b) TCRP1201+R300.

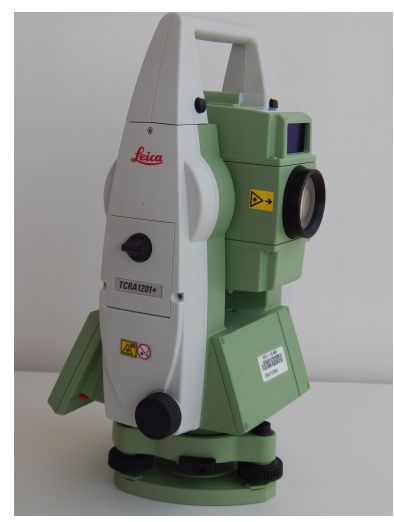

(c) TCRA1201+R400.

Figura 3.1: Estações Totais Robóticas utilizadas.

Os certificados de calibração das estações totais robóticas são apresentados no Anexo A, especificamente, no Anexo A.1 apresenta-se o certificado de calibração da TCA1201+, no Anexo 
A.2 apresenta-se o certificado de calibração da TCRP1201+R300 e, por fim, no Anexo A.3 apresenta-se o certificado de calibração da TCRA1201+R400.

\subsubsection{Base nivelante e carrier}

A base nivelante é a peça localizada na parte inferior de uma estação total robótica ou de um carrier. É por meio dela que ocorre a conexão do instrumento ou do prisma com o tripé ou com pilares de centragem forçada. O carrier é uma peça de conexão entre a base nivelante e o prisma refletor, cujos modelos de alta precisão contêm nível de bolha tubular para aumentar a acurácia da centragem e do nivelamento dos prismas nos pontos de interesse.

Para o desenvolvimento desta pesquisa foram utilizadas as bases nivelantes e os carrier indicados na sequência:

- Três bases nivelantes para os pilares de controles, de marca WILD Heerbrugg e modelo GDF22. Um exemplar pode ser visto na Figura 3.2a.

- Três bases nivelantes para os pilares de medição, de marca LEICA Geosystems e modelo GDF122. Um exemplar pode ser visto na Figura 3.2b.

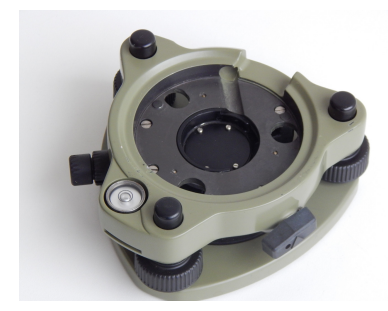

(a) Base WILD GDF22.

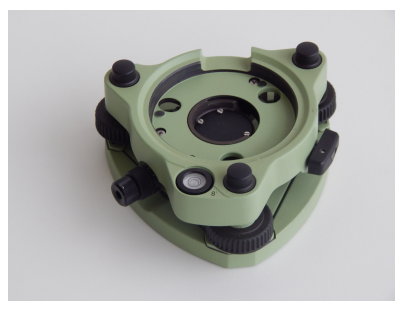

(b) Base Leica GDF122.

Figura 3.2: Bases nivelantes de precisão utilizadas.

A Figura 3.3 mostra os carrier que foram utilizados.

- Três carrier de marca WILD Heerbrugg e modelo GZR1, como mostra a Figura 3.3a.

- Três carrier de marca LEICA Geosystems e modelo GZR2, como mostra a Figura 3.3b.

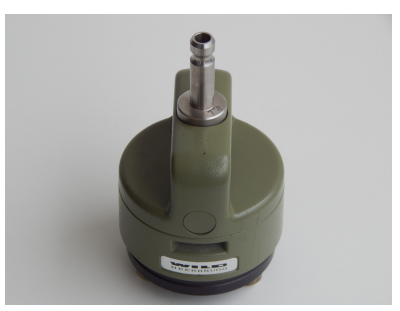

(a) Carrier GZR1.

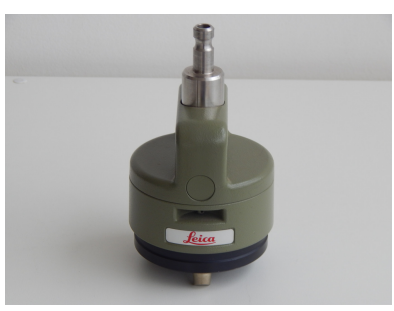

(b) Carrier GZR2.

Figura 3.3: Carrier de precisão utilizado. 


\subsubsection{Prisma refletor}

Dá-se o nome de prisma refletor ao acessório usado, em conjunto com uma estação total, para refletir o raio eletromagnético emitido pela mesma, durante a medição de uma distância. No caso do monitoramento geodésico com estações totais robóticas, eles são os alvos instalados sobre os pontos de monitoramento, cujas coordenadas serão determinadas para avaliar a deformação ou o deslocamento da mesma.

A qualidade da medição com um prisma circular depende de algumas características de fabricação, como a constante de adição do prisma, o desvio de paralelismo entre o raio incidente e o raio refletido, a qualidade da película de reflexão e do vidro. Dessa forma, apresentam-se os prismas refletores utilizados nesta pesquisa.

- Quatro prismas refletores circulares profissonais da marca LEICA Geosystems e modelo GPR111, com constante aditiva de $0,0 \mathrm{~mm}$, como mostra a Figura 3.4a.

- Dois prismas refletores circulares profissionais da marca LEICA Geosystems e modelo GPR121, com constante aditiva de 0,0 mm, como mostra a Figura 3.4b.

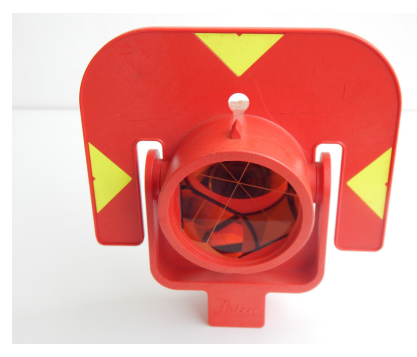

(a) Prisma GPR111.

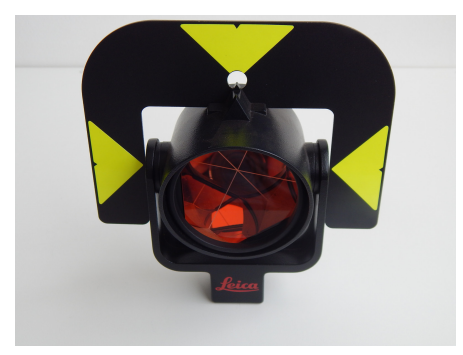

(b) Prisma GPR121.

Figura 3.4: Prismas refletores utilizados.

Com o grupo de acessórios utilizados na pesquisa foi possível montar seis combinações, formando duas composições, as quais contém base nivelante, carrier e prisma refletor, como mostra a Figura 3.5. Especificamente, a Figura 3.5a mostra a composição 1 e a Figura 3.5b mostra a composição 2. Os acessórios e materiais podem ser vistos com detalhe no manual da LEICA (2010).

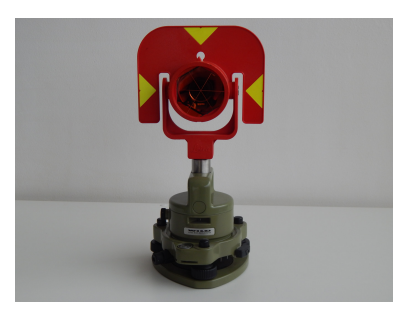

(a) Composição 1.

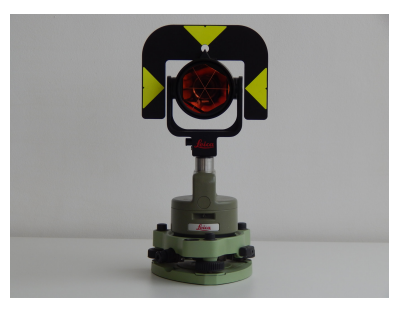

(b) Composição 2.

Figura 3.5: Composição de base nivelante, carrier e prisma refletor. 


\subsubsection{Tripé industrial}

Os tripés industriais são equipamentos projetados com maior peso e robustez que os tripés convencionais de madeira ou alumínio, cuja função é sustentar e posicionar os instrumentos de medição sobre os pontos determinados pelos operadores (ARMY, 2012).

Devido a sua robustez e facilidade de locomoção, eles são peças fundamentais para uso em medições de campo de alta precisão. No caso desta pesquisa, eles foram utilizados como pilares de centragem forçada para as medições com as estações totais robóticas e para o posicionamento dos prismas refletores dos pontos de controle.

Para o desenvolvimento desta pesquisa foram projetados e construídos dois tipos de tripés industriais:

- Dois tripés industriais metálicos e de centragem forçada. Como se pode ver na Figura 3.6a, estes tripés, que são as bases para as medições no processo de monitoramento, servirão para o suporte de duas estações totais robóticas.

- Três tripés industriais metálicos e de centragem forçada. Como se pode ver na Figura 3.6a, estes tripés, que são as bases para controle e verificação da estabilidade das bases de medições, servirão para o suporte dos prismas refletores.

Ressalta-se que uma das estações totais robóticas foi posicionada diretamente no perfil metálico externo do edifício Polo Terra, localizado no Campus 2 da USP São Carlos. Desta forma, completa-se a rede de seis pontos.

Com os instrumentos e os acessórios citados foi possível montar as três estações de medições e as três estações de controle, como mostra a Figura 3.6, para a materialização da rede topográfica espacial e a execução do processo de monitoramento.

A Figura 3.6a mostra a TCRP1021+ instalada em um tripé industrial de medição. A Figura 3.6b mostra uma das composições, contendo um prisma refletor GPR111, um carrier GZR1 e e uma base nivelante GDF22, instalados no tripé industrial de controle.

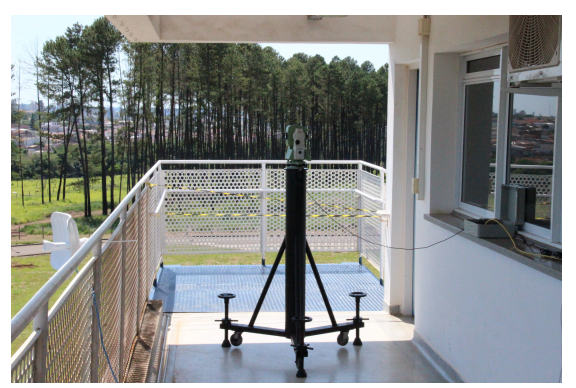

(a) Base de medição.

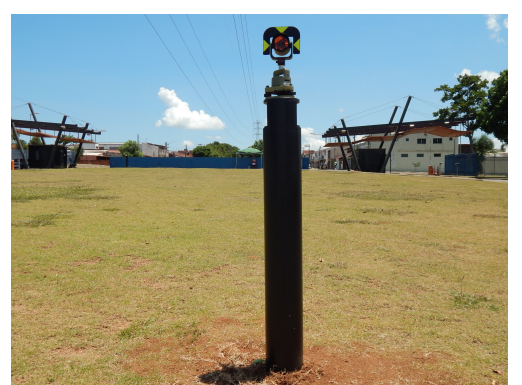

(b) Base de controle.

Figura 3.6: Tripés industriais da rede e de monitoramento. 


\subsubsection{Sensor metereológico}

A refração atmosférica é proporcional à dimensão da medição linear realizada, consequentemente, ela varia com a unidade de distância. O valor da distância medida por um distanciômetro eletrônico, Eletromagnetic Distance Measurement (EDM), é obtido, medindo o tempo de propagação das ondas eletromagnéticas através da atmosfera. A velocidade dessas ondas no vácuo $V_{0}$ é conhecida, porém, deve ser reduzida de acordo com a condição atmosférica do meio no qual as ondas se propagam no momento da medição. A velocidade da onda eletromagnética em um meio $V$ pode ser calculada, utilizando a equação (3.1), se o índice de refração $n$ for conhecido (RUEGER, 2012).

$$
V=\frac{V_{0}}{n}
$$

O valor de $n$ é sempre maior que a unidade e é afetado pela temperatura, pressão e vapor d'água contidos na atmosfera, bem como pelo comprimento de onda $\lambda$, das ondas eletromagnéticas transmitidas. Portanto, é necessário obter as medições dessas condições atmosféricas no momento das medições.

Segundo LEICA (2007), para o caso em que uma estação total robótica da série TPS 1200 é configurada no modo EDM de infravermelhos, o valor de $n$ é igual a 1,0002830 e o valor de $\lambda$, igual a $780 \mathrm{~nm}$. Este manual do fabricante afirma que o índice $n$ é calculado por meio da fórmula de Barrel \& Sears, sendo válido geralmente para pressão atmosférica igual a 1013, 25 mbar, temperatura do ar igual a $12^{\circ} \mathrm{C}$ e umidade relativa do ar, igual a $60 \%$. Nessas condições, a correção atmosférica é igual a zero.

A distância inclinada gravada no instrumento é a correta, se a correção da escala em ppm, $\mathrm{mm} / \mathrm{km}$, previamente definida, corresponder às condições atmosféricas existentes na altura da medição. A correção atmosférica inclui os ajustamentos para a pressão atmosférica, para a temperatura do ar e para a umidade relativa. Ainda, de acordo com o manual LEICA (2007), ressalta-se que a umidade do ar influencia a medição de distâncias, apenas se as condições atmosféricas forem extremamente quentes e úmidas.

Para medições em outras condições atmosféricas diferentes do padrão apresentado, é necessário corrigir o valor medido. A correção é feita de acordo com a fórmula empírica de Barrel \& Sears, indicada na equação (3.2).

$$
\Delta d=283,05-\left(\frac{0,2916 \cdot p}{(1+\alpha-t)}-\frac{4,126 \cdot 10^{-4} \cdot h}{(1+\alpha-t)} \cdot 10^{x}\right)
$$

Sendo,

$x=1 / 273,15$

$\alpha=(7,5 \cdot t /(237,3+t))+0,7857$ 
$\Delta d$ - correcção atmosférica em [ppm]

$p$ - pressão atmosférica em [mbar]

$t$ - temperatura do ar ambiente em $\left[{ }^{\circ} \mathrm{C}\right]$

$h$ - umidade relativa em [\%]

Assim, a distância inclinada pode ser corrigida, considerando o índice de refração, a onda eletromagnética, a temperatura, a pressão e a umidade, a qual pode ser calculada pela equação (3.3).

$$
d^{\prime}=d_{0}^{\prime} \cdot\left(1+p p m \cdot 10^{-6}\right)+m m
$$

Sendo,

$d^{\prime}$ - distância inclinada medida, corrigida em [m]

$d_{0}^{\prime}$ - distância inclinada medida, não corrigida em [m]

ppm - correção de escala em $[\mathrm{mm} / \mathrm{km}]$

$m m$ - constante de adição do prisma refletor em [mm]

Ressalta-se que as estações totais robóticas dispõem de rotinas operacionais internas, que realizam automaticamente as correções ppm, porém os dados atmosféricos coletados devem ser inseridos manualmente. Já no caso em que as estações totais robóticas são gerenciadas pelo software GeoMos, os dados atmosféricos devem ser coletados por sensores específicos e inseridos no sistema de forma automática e contínua. Estes dados atmosféricos são utilizados para o cálculo da correção ppm para que, posteriormente, sejam calculadas as correções das distâncias inclinadas medidas.

Para a coleta de dados metereológicos em tempo real, nesta pesquisa foi utilizado um sensor que realiza conjuntamente medições de temperatura e pressão, conforme a descrição a seguir:

- Sensor metereológico com coleta combinada de temperatura e pressão de marca STS e modelo DTM 101001, como pode ser visto na Figura 3.7.

Pressão: 500 a 1100 mbar

Temperatura: $-25{ }^{\circ} \mathrm{C}$ a $85{ }^{\circ} \mathrm{C}$

Out: RS232C

In: 5 a $30 \mathrm{~V}$ DC 


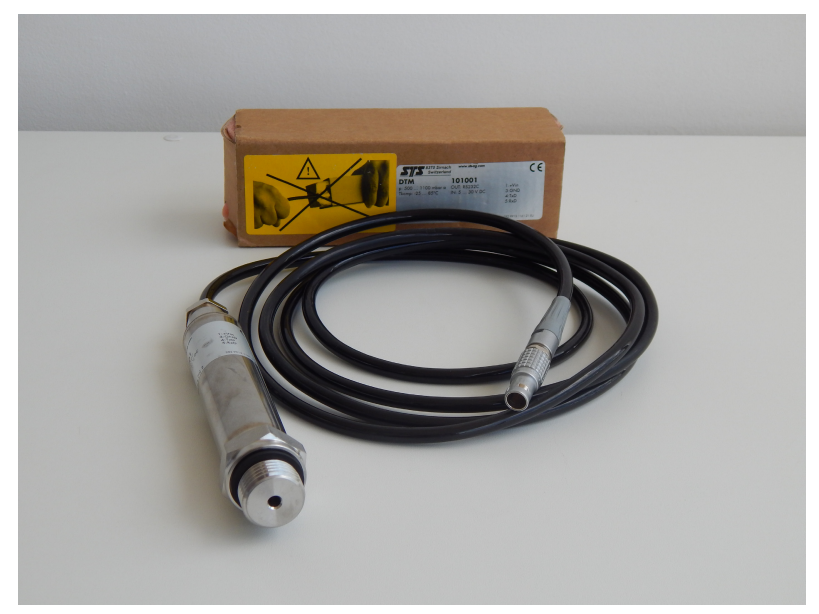

Figura 3.7: Sensor metereológico.

\subsubsection{Braço robótico}

Para validação do sistema e dos métodos propostos, é necessário controlar o alvo de monitoramento, para se obter as coordenadas espaciais do mesmo, pois, dessa forma, é possível simular a variação de suas coordenadas espaciais e, consequentemente, simular instabilidades estruturais.

Para que o processo de validação do sistema de monitoramento ocorra, ininterruptamente, 24 horas por dia, é fundamental que a simulação do deslocamento seja automatizada. Neste sentido, a solução apresentada foi a utilização de um braço robótico da marca UFACTORY e modelo uArm Swift Pro. Este instrumento executa as tarefas de variações das coordenadas espaciais $(X, Y, Z)$ de forma vetorial e com repetibilidade submilimétrica. A Figura 3.8 mostra os elementos descritivos do braço robótico utilizado. Especificamente, a Figura 3.8a mostra os componentes básicos do braço robótico uArm Swift Pro e a Figura 3.8b mostra a disposição de seus eixos coordenados.

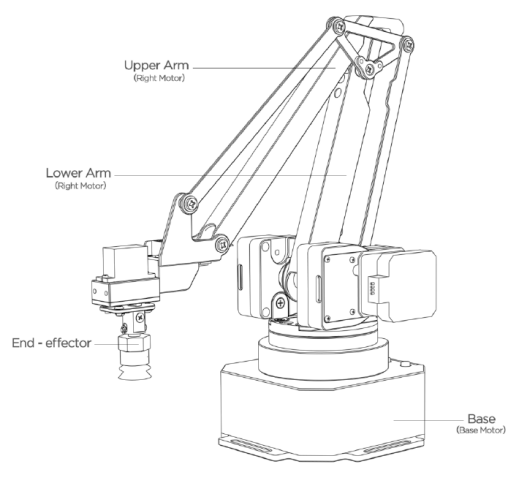

(a) Componentes do braço robótico.

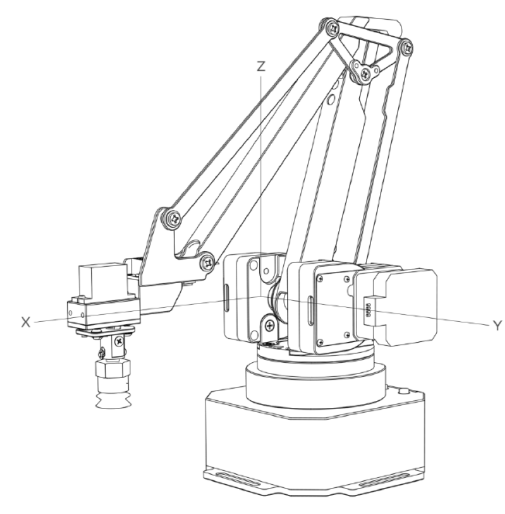

(b) Eixos do braço robótico.

Figura 3.8: Elementos descritivos do braço robótico.

A Figura 3.9 mostra o alcance e o intervalo de carga dinâmica do uArm Swift Pro. Nota-se aqui que a origem do sistema de coordenadas está localizada no centro da base do robô. 


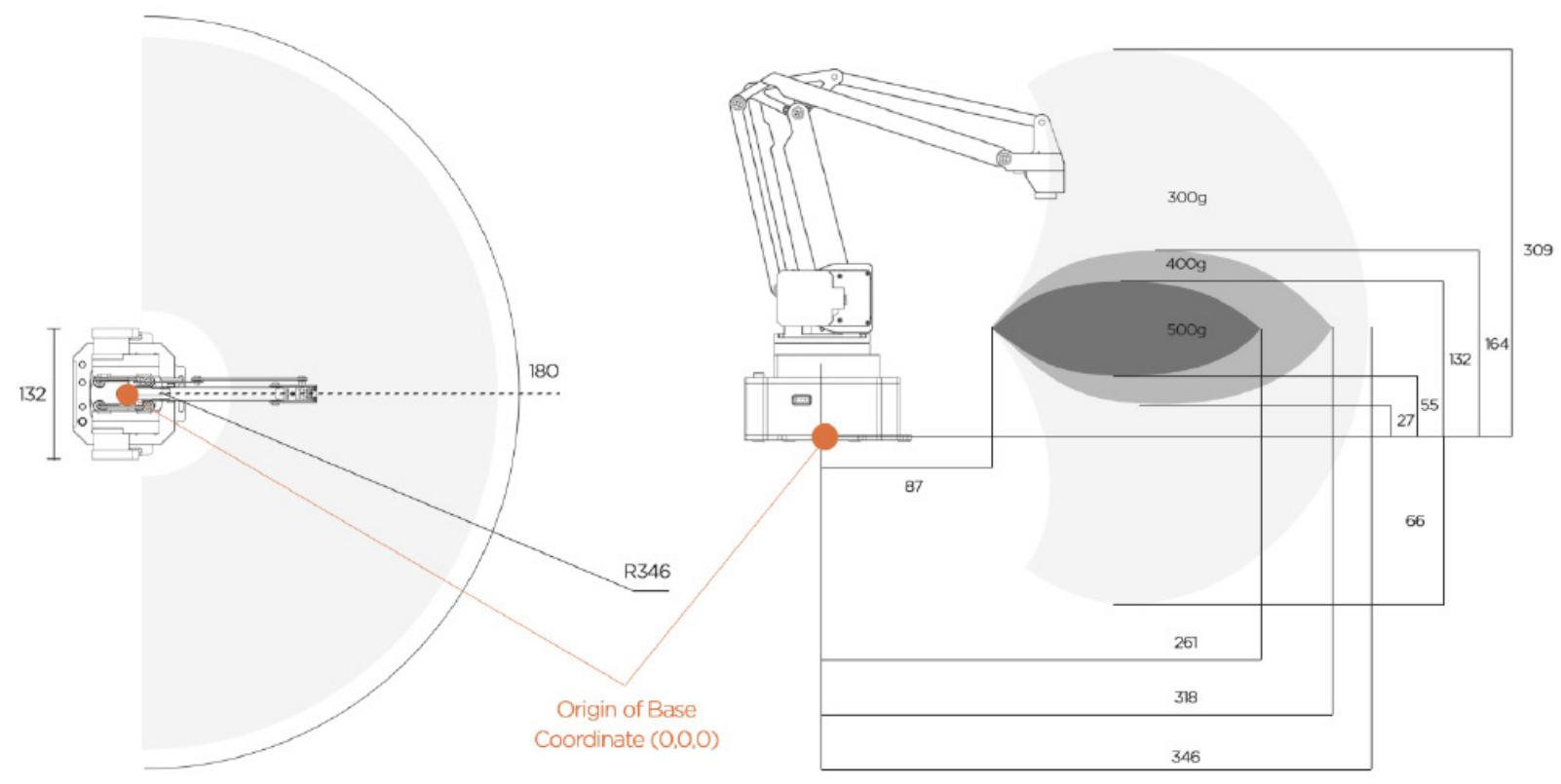

Figura 3.9: Alcance e intervalo de carga dinâmica braço robótico.

A Figura 3.10 mostra algumas das dimensões do uArm Swift Pro.

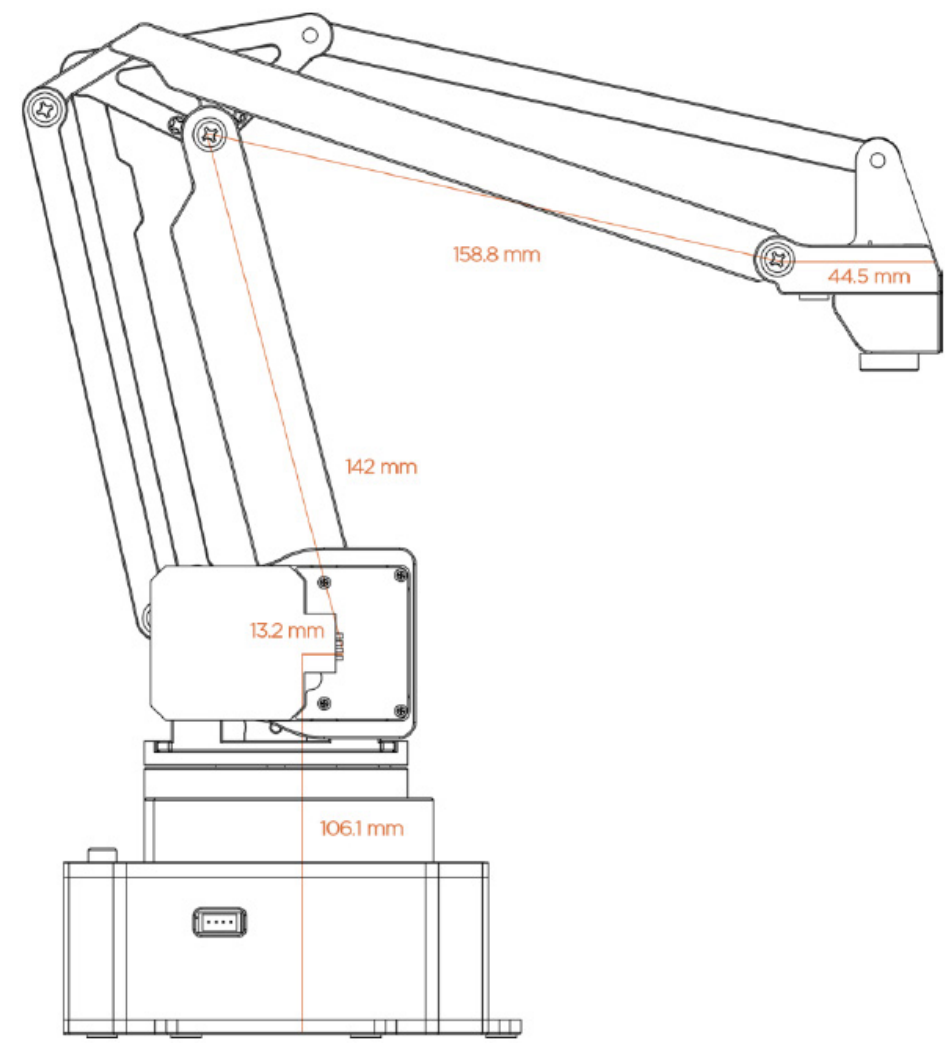

Figura 3.10: Dimensões do braço robótico em milímetros.

Para se apontar todas as especificações técnicas, referentes ao uArm Swift Pro, segue a Figura 3.11. 


\begin{tabular}{|c|c|c|c|c|}
\hline \multicolumn{5}{|c|}{ Specifications } \\
\hline Weight & \multicolumn{4}{|c|}{$2.2 \mathrm{~kg}$} \\
\hline Degrees of Freedom & \multicolumn{4}{|c|}{4} \\
\hline Repeatability & \multicolumn{4}{|c|}{$0.2 \mathrm{~mm}$} \\
\hline Max. Payload & \multicolumn{4}{|c|}{$500 \mathrm{~g}$} \\
\hline Working Range & \multicolumn{4}{|c|}{$50 \mathrm{~mm} \sim 320 \mathrm{~mm}$} \\
\hline Max. Speed & \multicolumn{4}{|c|}{$100 \mathrm{~mm} / \mathrm{s}$} \\
\hline Connector & \multicolumn{4}{|c|}{ Micro USB } \\
\hline Wireless & \multicolumn{4}{|c|}{ Bluetooth 4.0} \\
\hline Input Voltage & \multicolumn{4}{|c|}{$\mathrm{DC} 12 \mathrm{~V}$} \\
\hline Power Adapter & \multicolumn{4}{|c|}{ Input:100 240V 50/60Hz; Output: 12V5A 60W } \\
\hline $\begin{array}{l}\text { Operation Temperature \& } \\
\text { Humidity }\end{array}$ & \multicolumn{4}{|c|}{$\begin{array}{c}0^{\circ} \mathrm{C}-35^{\circ} \mathrm{C} \quad 30 \% \mathrm{RH}-80 \% \mathrm{RH} \\
\text { noncondensing }\end{array}$} \\
\hline $\begin{array}{c}\text { Storage Temperature \& } \\
\text { Humidity }\end{array}$ & \multicolumn{4}{|c|}{$\begin{array}{c}-20^{\circ} \mathrm{C}-60^{\circ} \mathrm{C} \quad 30 \% \mathrm{RH}-80 \% \mathrm{RH} \\
\text { noncondensing }\end{array}$} \\
\hline \multicolumn{5}{|c|}{ Hardware } \\
\hline Joint Type & \multicolumn{4}{|c|}{ Customized Gearbox + Stepper } \\
\hline Position Feedback & \multicolumn{4}{|c|}{12 bit Encoder } \\
\hline Reducer & \multicolumn{4}{|c|}{ Customized ultra-thin Gearbox } \\
\hline Dimension $\left(\mathrm{L}^{*} \mathrm{~W} * \mathrm{H}\right)$ & \multicolumn{4}{|c|}{$150 \mathrm{~mm}^{*} 140 \mathrm{~mm}^{*} 281 \mathrm{~mm}$} \\
\hline Mother Board & \multicolumn{4}{|c|}{ Arduino MEGA 2560} \\
\hline Material & \multicolumn{4}{|c|}{ Aluminum } \\
\hline Baud Rate & \multicolumn{4}{|c|}{$115200 \mathrm{bps}$} \\
\hline Extendable I/O Interface & \multicolumn{4}{|c|}{$\mathrm{I} / \mathrm{O}^{*} 27, \Pi \mathrm{\Pi} * 1,5 \mathrm{~V}^{*} 1,12 \mathrm{~V}^{*} 1$, Stepper*1 } \\
\hline \multicolumn{5}{|c|}{ Software } \\
\hline PC Control & \multicolumn{4}{|c|}{ uArm Studio } \\
\hline App Control & \multicolumn{4}{|c|}{ uArm Play } \\
\hline For Developer & \multicolumn{4}{|c|}{ Python/Arduino/ROS } \\
\hline Feature & \multicolumn{4}{|c|}{ Open Source } \\
\hline \multicolumn{5}{|c|}{ Joint Speed \& Torque } \\
\hline & Angle & Speed & Lifetime & Torque \\
\hline Base Motor & $0^{\circ} \sim 180^{\circ}$ & $40^{\circ} / \mathrm{s}$ & $>3000 \mathrm{~h}$ & $12 \mathrm{~kg} \cdot \mathrm{cm}$ \\
\hline Left Motor & $0^{\circ} \sim 130^{\circ}$ & $40^{\circ} / \mathrm{s}$ & $>3000 \mathrm{~h}$ & $12 \mathrm{~kg} \cdot \mathrm{cm}$ \\
\hline Right Motor & $0^{\circ} \sim 106^{\circ}$ & $40^{\circ} / \mathrm{s}$ & $>3000 \mathrm{~h}$ & $12 \mathrm{~kg} \cdot \mathrm{cm}$ \\
\hline End-effector Motor & $0^{\circ} \sim 180^{\circ}$ & $60^{\circ} / \mathrm{s}$ & $500 \mathrm{~h}$ & $2 \mathrm{~kg} \cdot \mathrm{cm}$ \\
\hline
\end{tabular}

Figura 3.11: Especificações do uArm Swift Pro.

Como a função do braço robótico é simular deslocamentos, o mesmo realiza estas tarefas, segurando um prisma refletor, e, para isso, foi utilizada a trava universal de que este robô dispõe. A Figura 3.12 mostra a trava universal presa ao end effector do braço.
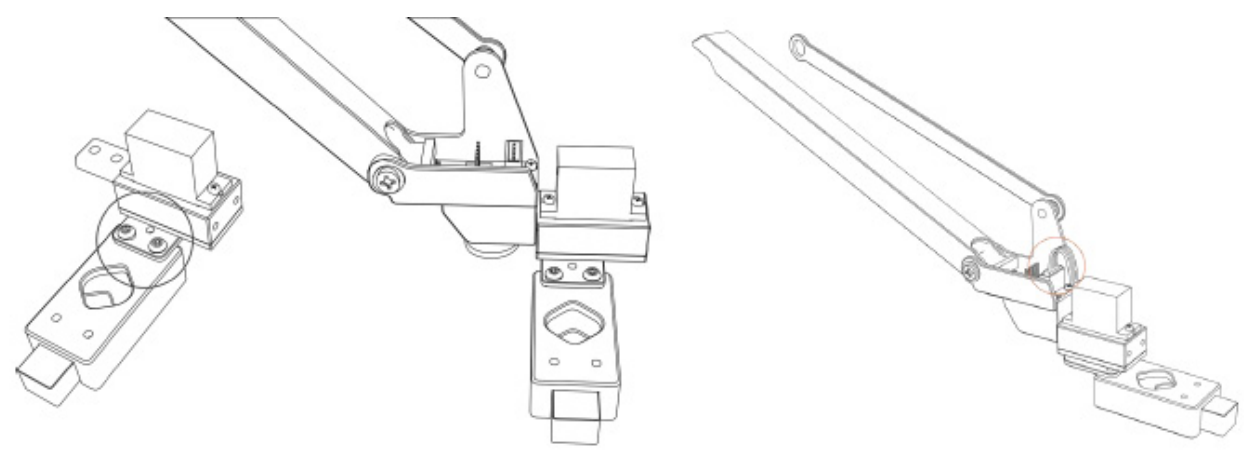

Figura 3.12: Trava universal presa ao end effector. 
A trava universal pode ser vista em detalhe na Figura 3.13.

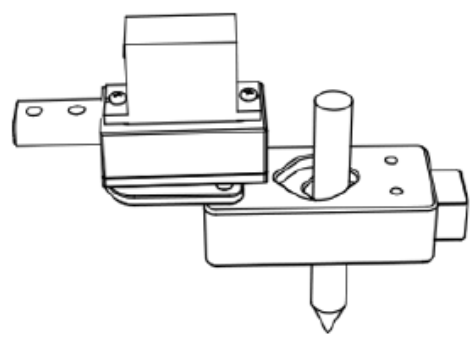

Figura 3.13: Trava universal, segurando um pino de acoplagem.

Ressalta-se que a composição do prisma refletor e o pino de acoplagem, a serem travados no braço robótico, pesam em torno de $420 \mathrm{~g}$, o que respeita o limite de carga. Os limites de atuação do braço foram respeitados, quando foram inseridas as variações de suas coordenadas espaciais dentro de seu espectro.

Dessa forma, para realizar o deslocamento controlado do alvo em três dimensões, e, assim, simular variações e instabilidades estruturais, foi utilizado um sistema para controle do alvo, como pode ser visto na Figura 3.14:

- Um braço robótico para simulação de alvo controlado uArm Swift Pro.

Um notebook contendo o software programado em Python para gerenciamento e controle do braço robótico.

- Um Nobreak de marca TS Shara e modelo Power UPS 4009 de 700VA.

O braço robótico uArm Swift Pro que foi utilizado para realizar o deslocamento do alvo e simular a instabilidade de uma estrutura pode ser visto na Figura 3.14. Na Figura 3.14a é mostrado somente o braço robótico, na Figura 3.14b pode ser visto o prisma refletor já instalado no braço robótico e na Figura 3.14c pode ser visto o sistema do robô.

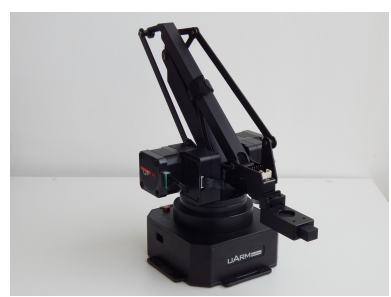

(a) Braço robótico.

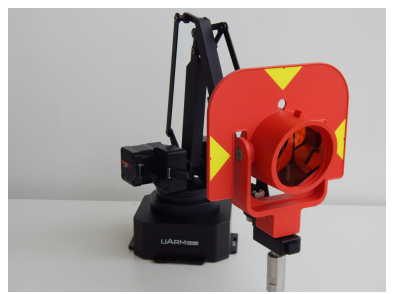

(b) Braço robótico e prisma.

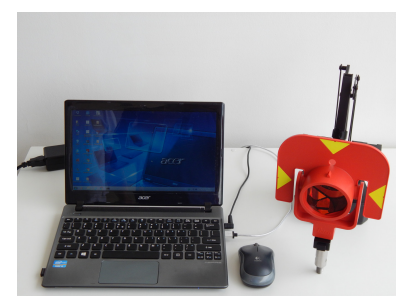

(c) Sistema do robô.

Figura 3.14: Sistema para controle do alvo. 


\subsection{Instrumentos utilizados no subsistema de comunicação}

Na elaboração do subsistema de comunicação foram desenvolvidas três caixas de comunicação, criadas para gerir a transferência e a energização de todas as estações totais robóticas utilizadas.

\subsubsection{Caixa de comunicação}

As caixas de comunicação que foram projetadas e construídas, nesta pesquisa, permitem operações em tempo real e, são basicamente, compostas por:

- Três Conversores de mídia entre os sinais dos instrumentos Porta Serial RS-232 e sinal de Ethernet (Serial Device Server) com Datalogger.

Um Conversor de marca Moxa e modelo 5110;

Um Conversor de marca USR e modelo USR-TCP232-410s;

Um Conversor de marca Netcom e modelo DS-42-W.

- Três Fontes chaveadas de 12V e 5A tipo colmeia de marca Tudoforte e modelo SL-1205A.

- Três Filtros de linha.

- Três Nobreaks de marca TS Shara e modelo Power UPS 4009 de 700VA.

- Três Cabos serial/lemo em Y para sinal e energia de marca LEICA Geosystems.

- Cabos de rede para comunicação e transmissão dos dados em tempo real.

Nas Figura 3.15, pode-se ver as três caixas de comunicação que foram confeccionadas para este subsistema. A Figura 3.15a mostra a caixa da TCA1201+. A Figura 3.15b mostra a caixa da TCRP1201+ e, por fim, a Figura 3.15c, a caixa da TCRA1201+.

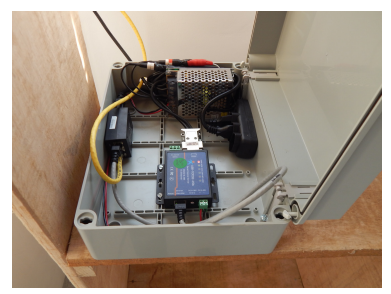

(a) Caixa para TCA1201+.

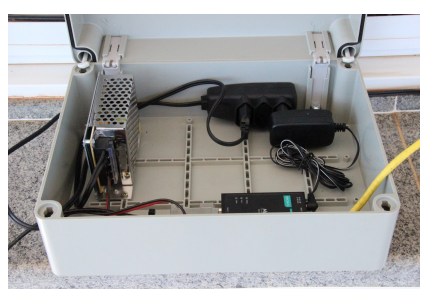

(b) Caixa para TCRP1201+.

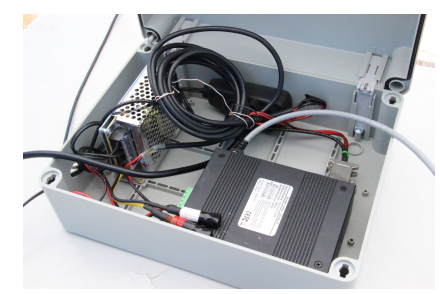

(c) Caixa para TCRA1201+.

Figura 3.15: Caixas de comunicação para as estações totais robóticas. 


\subsubsection{Transmissão de dados}

Para a transmissão e recepção de dados, gerados pelo subsistema de medição, foram utilizadas três antenas de rádio, sendo que, duas foram configuradas como clientes, para transmissão de sinal e uma, como principal, para recepção de sinal. As Figuras 3.16a e 3.16b mostram a antena em posições opostas. Já a Figura 3.16c mostra a caixa de fonte de energia e de conversão de mídia, com entrada e saída PoE/LAN.

- Três antenas de rádio de marca Ubiquiti e modelo LiteBeam M5.

Dimensões: 362 x 267 x $184 \mathrm{~mm}$

Peso: $720 \mathrm{~g}$

Consumo: $4 \mathrm{~W}$

Alimentação: Adaptador PoE 24V 0.2A

Interface de Rede: 1 porta ethernet 10/100Mbps

Ganho da antena: $23 \mathrm{dBi}$

Potência de Transmissão: $25 \mathrm{dBm}$

Frequência: 5150 a $5875 \mathrm{MHz}$

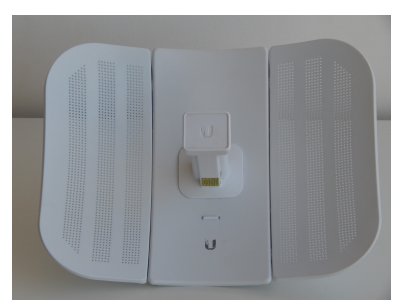

(a) Visão frontal.

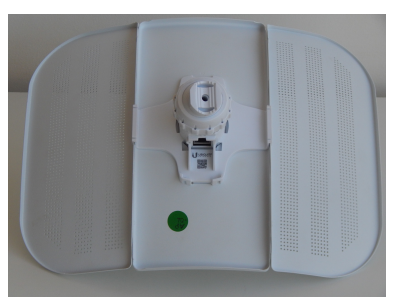

(b) Visão traseira.

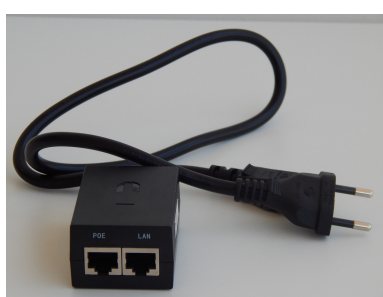

(c) Adaptador PoE.

Figura 3.16: Antenas e acessórios para transmissão de dados.

Logo que a antena principal recebe os dados, ela os envia diretamente, por meio de cabos de rede, para o switch da rede intranet, como mostra a Figura 3.17, o qual direciona todas as informações ao subsistema de processamento e alarme.

- Um switch de 8 portas de comunicação para gerenciamento de rede, aparelho de marca Encore e modelo ENH908-NWY, como pode ser visto na Figura 3.17a. A Figura 3.17b mostra um exemplar de cabo de rede utilizado. 


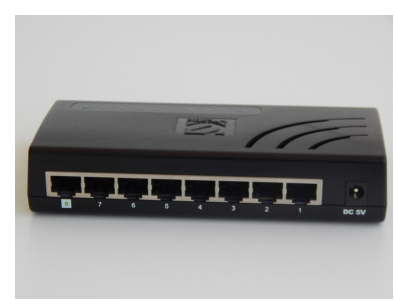

(a) Switch de rede.

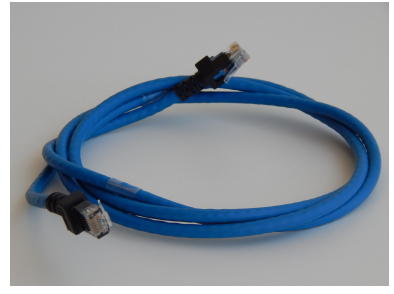

(b) Cabo de rede.

Figura 3.17: Comunicação da intranet.

\subsection{Instrumentos utilizados no subsistema de processamento e alarme}

Assim que os dados do subsistema de medição são gerados, os mesmos são transmitidos em tempo real pelo subsistema de comunicação até o subsistema de processamento e alarme. Dessa forma, os dados chegam diretamente aos softwares de processamento e gestão das medições.

\subsubsection{Software comercial}

Existem vários softwares comerciais que acompanham os instrumentos, sendo cada um dedicado a determinadas funções operacionais. No processo de monitoramento, é necessário utilizar aplicativos que proporcionem operações ininterruptas e com determinada periodicidade. Dessa forma, nesta pesquisa foram utilizados:

- Um software LEICA Geo Office (LGO) versão 5.0 para descarga dos dados brutos observados em campo pela estação total robótica TCRA120+, a qual foi aleatoriamente escolhida para realizar as medições da rede topográfica espacial.

- Um software LEICA GeoMoS Monitor (GeoMos) versão 6.3 para operação e gestão das três estações totais robóticas e o sensor metereológico, além de realizar o monitoramento e a geração dos dados brutos. Este software, portanto, realiza todo o controle operacional destes instrumentos.

\subsubsection{Software aberto}

A aquisição, o armazenamento e o processamento das informações coletadas em campo foram fatores preponderantes para a realização das atribuições do sistema de monitoramento proposto. A coleta de informações, nestes casos, foi executada por meio de medições realizadas por meio de diferentes instrumentos. Como, a priori, esses dados se encontravam em sua forma bruta, contendo apenas as observações, tornou-se necessário definir um sistema de busca, de estruturação e de processamento, para que fosse disponibilizado ao operador um conjunto de dados, de forma organizada, rápida e confiável. Com o objetivo de gerenciar esse universo de informações foi desenvolvido um sistema de gerenciamento de dados para a aplicação dos 
métodos de determinação de coordenadas espaciais e, também, para o sistema operacional do braço robótico. Para isso, esse sistema se baseia em referenciadores duplamente indexados que formam um conjunto de índices de controle. A escolha dos atributos indexadores é realizada por meio de uma chave primária, representativa e comum a todos os elementos. Portanto, trata-se de um sistema de gerenciamento de dados brutos, oriundos de medições de pontos monitorados, que permite o acesso rápido e sem dubiedades para a sua utilização.

É importante salientar que, aos dados de medições das estações totais robóticas, são atribuídos os identificadores $I D$, assim como, dos pontos levantados, dos valores de direções angulares horizontais, e dos ângulos verticais e das distâncias inclinadas. Nesse caso, além dos valores de medição indicados, pode-se também adicionar atributos e códigos para o objeto medido, a exemplo de dados da pressão atmosférica, da temperatura, dos identificadores do ponto de estação e de orientação das coordenadas calculadas em campo e outros. Tem-se, assim, um conjunto de informações que podem ser listadas e ordenadas, por meio do uso de softwares, a exemplo do GeoMos e do LGO.

Os ditos softwares de monitoramento, por sua vez, estão configurados para lerem a tabela de dados ordenados, segundo uma configuração de leitura de dados individuais. Cada programador define as suas prioridades em função do seu conceito de eficiência do software. Todavia, não existe um padrão de formatação que permita o intercâmbio eficiente de dados.

Nesse sentido, é necessário estabelecer um sistema de gerenciamento de dados espaciais, que permita o armazenamento, a manipulação e a disponibilização dos dados de forma eficiente, tanto para os softwares comerciais, quanto para os aplicativos especializados, criados por usuários individuais. Assim, o foco deve ser a facilidade de tomada de decisão em diferentes âmbitos da aplicação dos dados coletados. Para tal realização, foi utilizado:

- Um software Python versão 3.1 de código livre e aberto, para elaboração das programações operacionais do braço robótico e dos métodos de determinações de coordenadas espaciais para a rede topográfica espacial e para determinação das coordenadas espaciais do processo de monitoramento. 


\section{Capítulo}

\section{TÉCNICAS DE MEDIÇÃO PARA O MONITORAMENTO GEODÉSICO DE ESTRUTURAS}

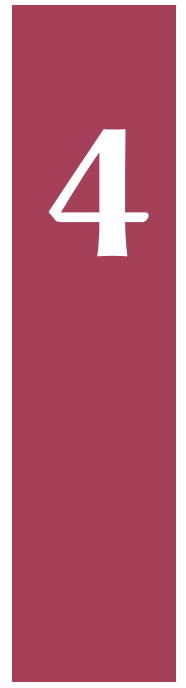

As técnicas de medição utilizadas no monitoramento geodésico de estruturas são basicamente as mesmas utilizadas nos levantamentos topográficos e geodésicos convencionais. As características que as diferenciam estão relacionadas ao nível de precisão exigido nas medições e a combinação entre diferentes tipos de instrumentos em uma mesma medição (EHIGIATOR; ASHRAF, 2012).

As técnicas de medição aplicadas no monitoramento geodésico baseiam-se em três princípios de medições geodésicas, que são:

- Medições angulares e lineares;

- Medições baseadas em processos fotogramétricos;

- Medições baseadas na tecnologia GNSS;

- Medições baseadas no uso de escâneres a laser.

Devido ao objeto de estudo desta tese, discutem-se, aqui, somente as principais características das técnicas de medições angulares e lineares e o seu emprego no monitoramento geodésico de estruturas.

\subsection{Técnicas de medições angulares e lineares}

O emprego destas técnicas de medições, em monitoramento geodésico de estruturas, remonta ao procedimento clássico para o monitoramento geodésico, por meio de técnicas que consistem em dividir as medições em duas etapas independentes, que compreendem as medições 
dos deslocamentos horizontais e dos verticais, de dois grupos distintos de pontos da estrutura (BINGLEY, 1990). Os deslocamentos horizontais são verificados, em função da determinação das coordenadas planimétricas dos pontos de monitoramento, por meio do emprego de técnicas de triangulação, de trilateração, de triangulateração ou de poligonação. Em todos os métodos de medição indicados acima, medem-se ângulos e distâncias por meio de estações totais, instaladas sobre os pontos de referência e visando o ponto de monitoramento.

Já os deslocamentos verticais são verificados, em função da determinação das cotas dos pontos de monitoramento, ou por meio de um nivelamento geométrico com o uso de um nível mecânico ou digital de alta precisão, o qual realiza medição da distância vertical, ou por meio de nivelamento trigonométrico com o uso de estações totais, a qual realiza medições angulares e lineares. A rede planimétrica compõe-se da rede de pontos de referência, da rede de pontos de controle e dos pontos de monitoramento, sendo que a rede de pontos de controle e a rede de pontos de referência são estabelecidas utilizando as mesmas técnicas de medições (OGUNDARE, 2018).

\subsection{Determinação planimétrica da rede}

A rede de pontos de controle é o coração do monitoramento. Os pontos dessa rede formam o arcabouço da estrutura de monitoramento. Eles devem ser, portanto, totalmente estáticos e localizados fora da área de influência da estrutura, ou seja, eles não devem, de nenhuma forma, sofrer variações de posição devido aos efeitos dinâmicos da estrutura monitorada.

A rede de pontos de referência é constituída pelos pontos de medição, que são as estações que monitoram os pontos da estrutura. Por estarem, geralmente, dentro da área de influência da estrutura, eles podem sofrer alterações nas suas posições. Por esta razão, eles são monitorados pelos pontos de controle.

Após a instalação de todos os pontos, incluindo os pontos de monitoramento, as redes são calculadas e ajustadas em planimetria e altimetria, simultaneamente. Tem-se, assim, o instante zero da posição de todos os pontos. Nas campanhas de medições sucessivas, antes de cada medição de um ponto de monitoramento, verifica-se a posição do ponto de referência correspondente. No caso de movimentação, os valores obtidos são considerados no posicionamento dos pontos de monitoramento, medidos a partir dessa estação (LAMBROU; PANTAZIS; NIKOLITSAS, 2011). Apresenta-se, a seguir, uma breve descrição sobre os tipos de redes de medições topográficas, utilizadas para o estabelecimento dos pontos de controle e dos pontos de referência de um monitoramento geodésico.

\subsubsection{Rede de Triangulação}

Uma rede de triangulação consiste de uma série de triângulos justapostos, conforme indicado na Figura 4.1. A consistência geométrica se dá pela medição de uma base, por exemplo, o lado 
$\overline{12}$ da figura e dos ângulos internos de cada triângulo. De acordo com os valores dos ângulos e da base medida, obtêm-se os valores das coordenadas de cada vértice dos triângulos (ISO171233, 2001). Por se tratar de uma rede de triângulos, o processamento é realizado, aplicandose técnicas de ajustamento pelo Método dos Mínimos Quadrados (MMQ). Têm-se assim as coordenadas planimétricas ajustadas de todos os vértices dos triângulos.

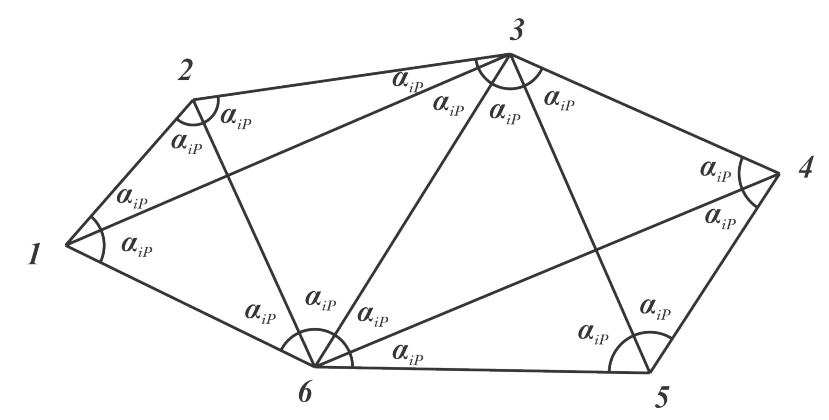

Figura 4.1: Rede de Triangulação.

Sendo,

$\alpha_{i P}$ - ângulos horizontais entre os pontos $I$ e $P$

O maior inimigo da precisão angular é a refração atmosférica. Por essa razão, devem-se realizar as devidas correções nas medições dos ângulos.

\subsubsection{Rede de Trilateração}

Uma rede de trilateração também consiste de uma série de triângulos justapostos, conforme indicado na Figura 4.2. A diferença, neste caso, é que ao invés de se medir os ângulos internos de cada triângulo, medem-se os comprimentos dos lados, que devem estar em conformidade com a ISO17123-4 (2001). O processamento da rede também é feito aplicando-se técnicas de ajustamento pelo MMQ.

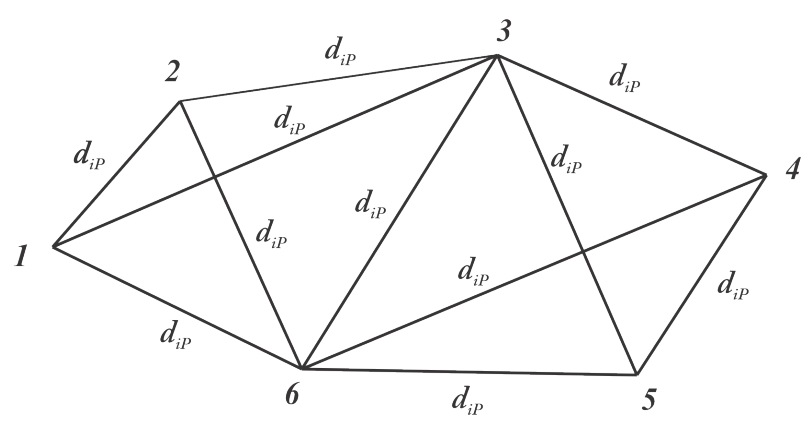

Figura 4.2: Rede de Trilateração.

Sendo,

$d_{i P}$ - distâncias entre os pontos de uma estação base $I$ e uma estação a vante $P$ 
Durante muito tempo, as medições angulares foram mais precisas do que as medições de distâncias. Por esta razão, dava-se preferência ao uso do método de triangulação. A partir da década de 60, entretanto, com o advento dos medidores eletrônicos de distância, a medição linear passou a ter precisão compatível com as medições angulares e, como consequência, as redes de trilateração passaram a ser cada vez mais importantes para o monitoramento de estruturas.

\subsubsection{Rede de Triangulateração}

Uma rede de triangulateração combina uma rede de triangulação com uma rede de trilateração para produzir um sistema de controle, no qual todos os ângulos e distâncias são observados, como ilustra a Figura 4.3. Portanto, trata-se de um sistema mais completo que os mencionados anteriormente, por apresentar todas as vantagens de ambos, dos quais é derivado. As vantagens deste tipo de rede é o aumento das observações que, consequentemente, aumenta a redundância, o que facilita estimativas e detecção de possíveis erros, tornando o método robusto (KHAMEN; FAIG, 1988).

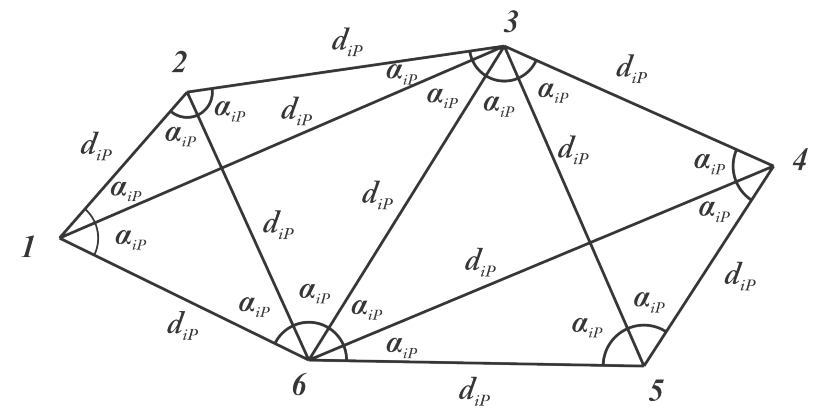

Figura 4.3: Rede de triangulateração.

Sendo,

$\alpha_{i P}$ - ângulos horizontais entre os pontos de uma estação base $I$ e de uma estação a vante $P$

$d_{i P}$ - distâncias entre os pontos de uma estação base $I$ e de uma estação a vante $P$

\subsubsection{Poligonação}

A poligonação é um método consagrado de implantação de pontos de apoio topográficos. Ele consiste, basicamente, em medir ângulos e distâncias sucessivas em um caminhamento sobre o terreno, realizando um transporte de coordenadas, de acordo com métodos de cálculos topométricos convencionais da topografia (GHILANI, 2018). Por meio do encadeamento das medições angulares e lineares, determinam-se as coordenadas dos pontos de apoio, conforme indicado na Figura 4.4. 


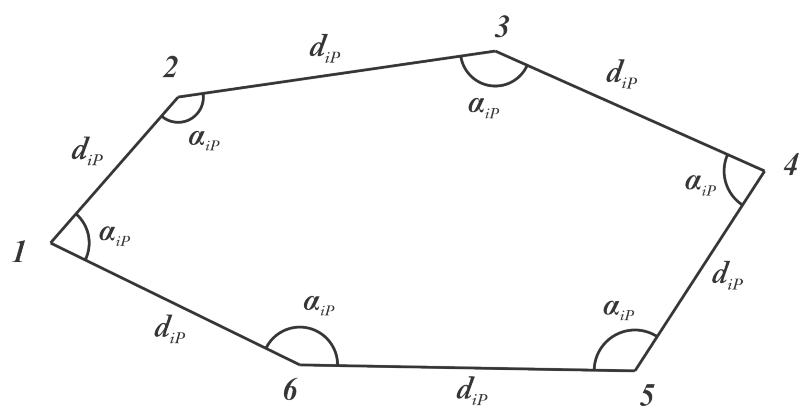

Figura 4.4: Poligonal topográfica.

Sendo,

$\alpha_{i P}$ - ângulos horizontais entre os pontos de uma estação base $I$ e de uma estação a vante $P$ $d_{i P}$ - distâncias entre os pontos de uma estação base $I$ e de uma estação a vante $P$

No caso de poligonais para monitoramento, elas são sempre topograficamente fechadas e, portanto, geometricamente definidas e restritas a regras matemáticas que permitem que se possa determinar a consistência entre os valores medidos e os valores geométricos calculados. As diferenças entre eles representam os erros de fechamento da poligonal, os quais são considerados como parâmetros de controle da qualidade da mesma. Os erros, assim determinados, são comparados com valores de tolerâncias predefinidos e, se aprovados, são compensados, em função das grandezas medidas. A poligonal, assim compensada, torna-se geometricamente consistente, para que se possa calcular as coordenadas dos seus vértices.

Geralmente, o uso do método de medição por poligonação é adotado para grandes estruturas, ou para o monitoramento de pontos instalados em galerias internas da estrutura, em que não é possível usar outros métodos de medição. As medições, a partir de poligonação, são sempre realizadas por meio de procedimentos de centragem forçada, instalando o instrumento topográfico, diretamente sobre o ponto de monitoramento.

\subsection{Determinação altimétrica da rede}

A determinação das cotas dos pontos de controle e de referência é realizada por meio de um nivelamento geométrico ou trigonométrico, ambos de precisão, conforme são descritos a seguir.

\subsubsection{Nivelamento geométrico}

O nivelamento geométrico determina as diferenças de altitudes ou de cotas entre pontos, por meio da medição da distância vertical entre cada um deles e de um plano horizontal de referência, a partir de um nível. Quando o instrumento é estacionado e nivelado sobre o terreno, de maneira que o seu eixo vertical coincida com a vertical do lugar, a luneta estabelece um plano horizontal. O nível assim instalado, em conjunto com réguas graduadas, permite realizar me- 
dições de alturas (ISO17123-2, 2001). As distâncias verticais são medidas a partir da leitura do ponto de interseção do plano horizontal com a régua graduada, instalada sobre os pontos de interesse de nivelamento, como mostra a Figura 4.5

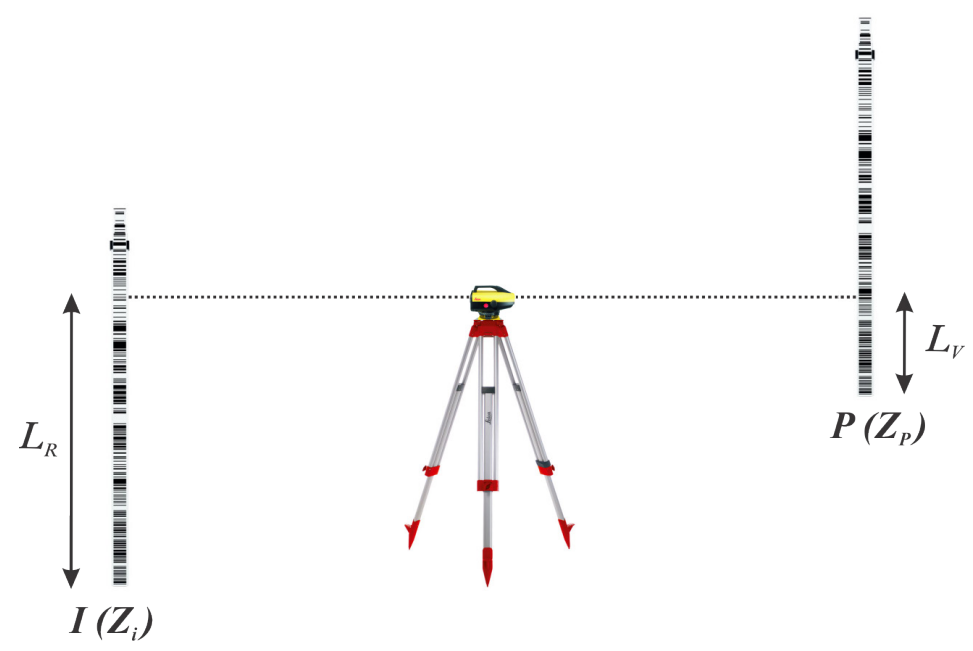

Figura 4.5: Princípio básico de um nivelamento geométrico.

Se a altitude ponto $I$ for conhecida, obtém-se a altitude do ponto $P$, pela equação (4.1)

$$
Z_{P}=Z_{i}+\left(L_{R}-L_{V}\right)
$$

Sendo,

$Z_{i}$ - altitude do ponto $I$

$Z_{P}$ - altitude do ponto $P$

$L_{R}$ - leitura de ré

$L_{V}$ - leitura de vante

Além disso, durante o processo de medição em campo, devem-se evitar algumas ocorrências, tais como,

- Vibrações nas proximidades do local, onde o instrumento será instalado;

- Instabilidade dos pontos de nivelamento;

- Exposição excessiva à luz do sol.

Devem-se, também, efetuar séries de observações para controlar a qualidade dos valores observados, que são calculados pela média das observações. Para a escolha do instrumento de medição, devem-se utilizar níveis digitais, que melhoram os resultados obtidos. 
Conforme já citado, o nivelamento geométrico pode também ser aplicado para o monitoramento do deslocamento vertical de pontos de monitoramento da estrutura. Nesses casos, o procedimento de trabalho de campo é o mesmo de um nivelamento de precisão.

\subsubsection{Nivelamento trigonométrico}

O nivelamento trigonométrico para monitoramento de estruturas deve ser de precisão, o qual envolve observações de ângulos verticais zenitais ou de altura e distâncias horizontais ou inclinadas entre dois pontos e esta operação visa à determinação da diferença de nível entre eles, como pode ser visto na Figura 4.6. Sucintamente, a diferença de altura entre os pontos é obtida, por meio da resolução de triângulos e, devido a isso, a elevação é determinada indiretamente (ISO17123-1, 2002).

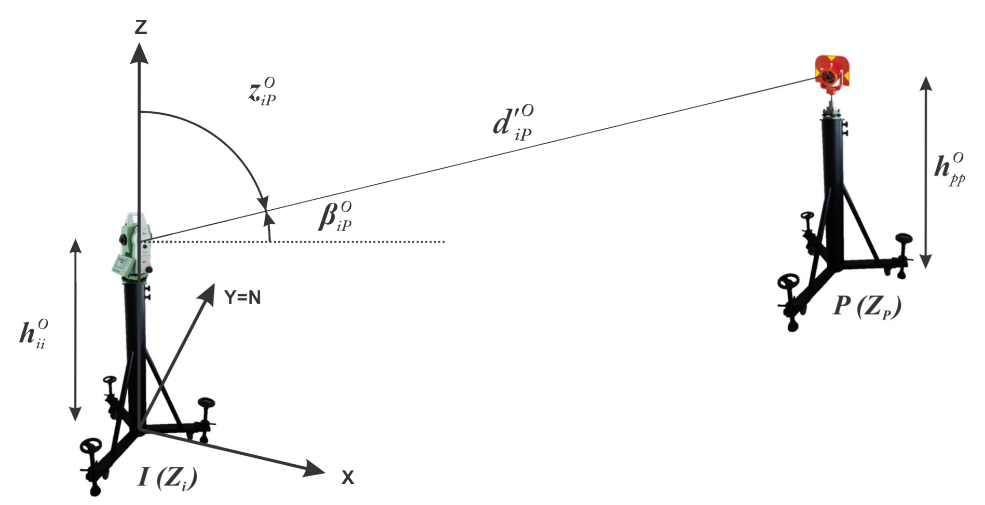

Figura 4.6: Relação geométrica de um nivelamento trigonométrico.

Se a altitude ponto $I$ for conhecida, obtém-se a altitude do ponto $P$, pela equação (4.2).

$$
Z_{P}=Z_{i}+\left(\left(d_{i P}^{O} \cdot \operatorname{sen}\left(\beta_{i P}^{O}\right)\right)+\left(h_{i i}^{O}-h_{p p}^{O}\right)\right)
$$

Sendo,

$Z_{i}$ - altitude do ponto $I$

$Z_{P}$ - altitude do ponto $P$

$z_{i P}^{O}$ - ângulos vertical zenital medido entre os pontos $i P$

$\beta_{i P}^{O}$ - ângulos vertical de altura medido entre os pontos $i P$

$d_{i P}^{O}$ - distâncias inclinada medida entre os pontos pontos $i P$

$h_{i i}^{O}$ - altura do instrumento no ponto $I$

$h_{p p}^{O}$ - altura do prisma no ponto $P$

Com o advento das estações totais robóticas, o nivelamento trigonométrico tornou-se um método ágil, com precisões comparáveis aos dados obtidos por nivelamentos geométricos, com os quais se determinam as elevações diretamente. Mas, devem-se considerar influências de 
alguns fatores na propagação dos erros, como a obtenção da altura do instrumento, os efeitos atmosféricos, o posicionamento e a altura dos alvos, a precisão dos equipamentos bem como as distâncias de visadas.

Portanto, pode ser utilizado para o estabelecimento de redes de controle vertical para trabalhos fotogramétricos, topográficos e também podem ser referência para a determinação de coordenadas altimétricas de pontos engastados em pontes, edifícios, barragens e outras grandes estruturas, com a finalidade de analisar deslocamentos estruturais (ANDERSON; MIKHAIL, 1998).

\subsection{Determinação da estabilidade da rede}

\subsubsection{Rede topográfica espacial de referência absoluta}

Para monitoramento contínuo de um corpo estrutural, por meio das técnicas geodésicas, são necessárias medições periódicas dos prismas refletores de monitoramento, com possível instabilidade, por meio de uma rede de pontos de referência, localizados externamente às estruturas, em um local com maior estabilidade, trata, assim, de uma rede de referência absoluta (ARMY, 1994). Dessa forma, deve-se ainda verificar os pontos da rede de referência para controlar e garantir suas estabilidades e, também, identificar possíveis pontos com valores que ultrapassem a tolerância predefinida para não induzir o sistema a gerar informações errôneas. $O$ fato de os instrumentos de medições estarem estrategicamente localizados em posições estáveis e inertes às movimentações de uma estrutura, pode elevar, não apenas às precisões, mas também a acurácia, o que leva a obter informações mais confiáveis (FANGI, 2008).

A Figura 4.7 mostra a rede espacial topográfica de referência absoluta, com três estações totais robóticas instaladas nas estações de referência e três prismas refletores instalados nos pontos de controle, ambos posicionados externamente ao objeto a ser monitorado (CHEN; CHRZANOWSKI; SECORD, 1990).

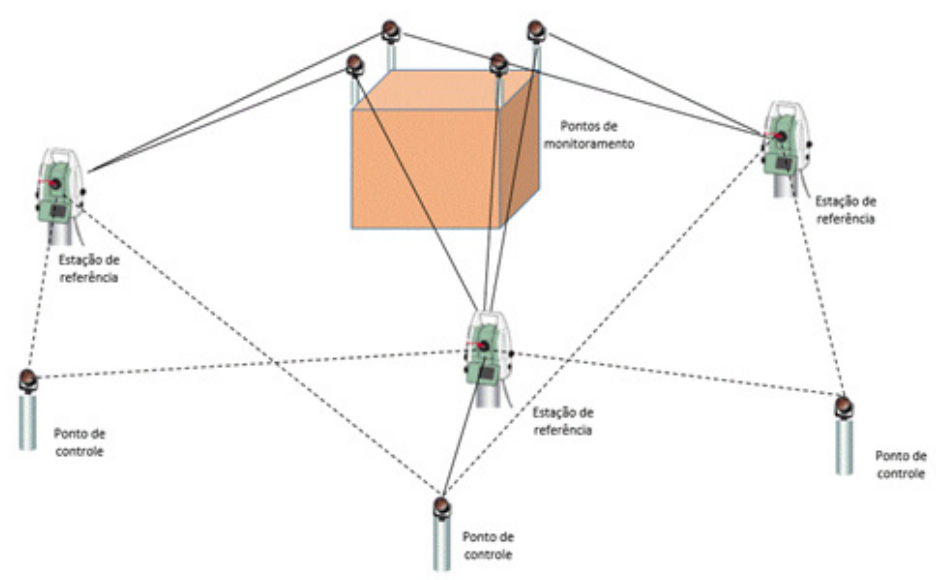

Figura 4.7: Rede topográfica espacial de referência absoluta. 
Dessa forma, para a verificação da estabilidade das estações de referência, antes de se realizar as medições dos pontos de monitoramento, as três estações totais robóticas realizam medições relativas aos três pontos de controle e, por meio do método de recessão espacial, determinam-se, no instante, as coordenadas espaciais das estações de referência. Ressalta-se que este procedimento deve ser efetuado em todo o processo de monitoramento.

\subsubsection{Recessão espacial}

No método de recessão espacial, também conhecido como estação livre, o equipamento é instalado sobre o ponto em que as coordenadas devem ser determinadas e verificadas e, assim, são realizadas observações, objetivando-se os pontos de referência. Mas, para isso, deve-se efetuar, no mínimo, a observação de dois pontos de controle para a determinação das coordenadas espaciais de interesse.

Para a realização dos cálculos deste método, os instrumentos foram configurados no software GeoMos, com um de seus aplicativos, a estação livre, também conhecida por free station. Este aplicativo utiliza um algoritmo embasado pela lei dos senos, cossenos e princípios do MMQ para calcular o ajustamento das observações entre as medições e as coordenadas dos pontos de referência. Este tipo de modelagem matemática permite calcular as coordenadas espaciais do ponto da estação seguido de suas respectivas precisões (LEICA, 2007).

Antes de armazenar o resultado da operação é possível analisar por meio do software GeoMos os resíduos das medições para os pontos, a fim de controlar a recessão espacial e, consequentemente, a estabilidade das bases.

O método de posicionamento de recessão espacial determina a coordenada espacial da estação de referência $\left(X_{i}, Y_{i}, Z_{i}\right)$, medindo direções horizontais $\alpha_{i R_{1,2,3}}^{O}$, ângulos verticais $\beta_{i R_{1,2,3}}^{O} \mathrm{e}$ distâncias inclinadas $d_{i R_{1,2,3}}^{\prime O}$ relativas a três estações de controle $R_{1}, R_{2}$ e $R_{3}$, conforme indicado na Figura 4.8.

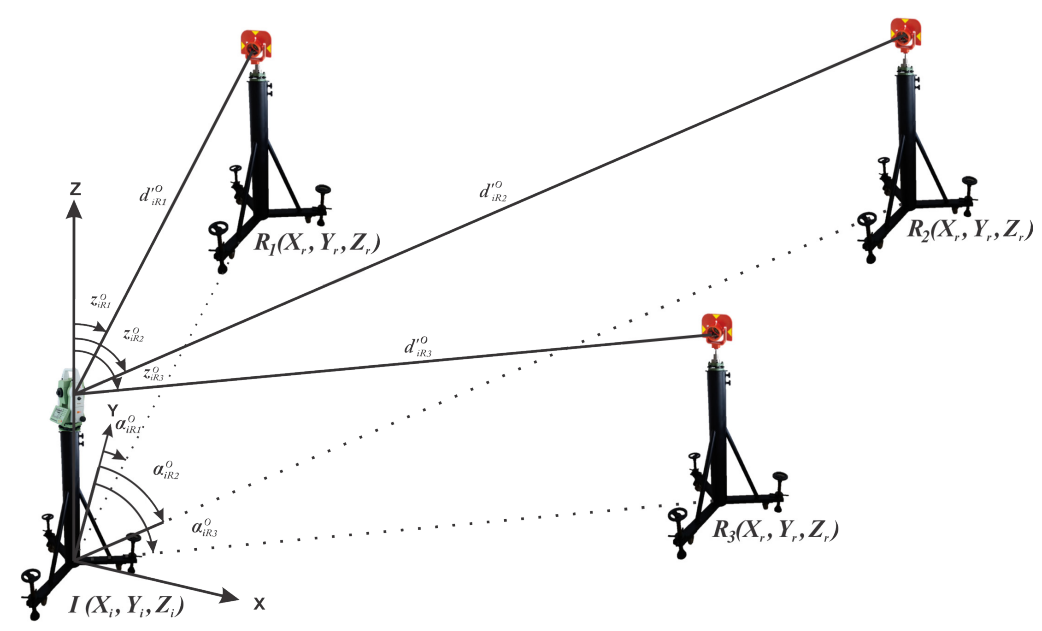

Figura 4.8: Relação geométrica de uma recessão espacial. 
Sendo,

$X_{i}, Y_{i}, Z_{i}$ - coordenadas espaciais a serem determinadas para o ponto $I$

$X_{r}, Y_{r}, Z_{r}$ - coordenadas espaciais conhecidas do ponto de orientação $R$

$\alpha_{i R}^{O}$ - ângulo horizontal observado entre os pontos $I$ e $P$

$z_{i R}^{O}$ - ângulos vertical zenital observado entre os pontos $I$ e $R$

$d_{i R}^{\prime O}$ - distância inclinada observada entre os pontos $I$ e $R$

É importante considerar que todas as estações totais robóticas possuem aplicativos embutidos para o processo de estação livre. No caso em que tais estações são operadas pelo software GeoMos, este realiza todas as operações da recessão espacial para a determinação das coordenadas espaciais desconhecidas das bases $\left(X_{i}, Y_{i}, Z_{i}\right)$.

\subsection{Ajustamento das observações}

\subsubsection{Método paramétrico}

Devido ao fato de vários métodos de determinação de coordenadas, apresentados na sequência deste capítulo, utilizar o método de ajustamento paramétrico de observações, apresenta-se, a seguir, um resumo do desenvolvimento matemático deste método, para melhor compreensão das formulações matemáticas aplicadas ao longo dos próximos capítulos.

O Método de Ajustamento Paramétrico ou de Observações Indiretas, como o próprio nome diz, baseia-se no estabelecimento de um conjunto de equações de observações, expressas em função das incógnitas, que não foram diretamente medidas, mas que se relacionam com as grandezas observadas (GHILANI, 2017). Cada medição produzirá uma equação de observação do tipo,

$$
l_{i}=f_{i}\left(X_{1}, X_{2}, X_{3}, \cdots, X_{u}\right)
$$

Sendo,

$l_{i}$ - valor medido da observação $(i)$

$X_{1}, X_{2}, X_{3}, \cdots, X_{u}$ - incógnitas da equação

$i=1,2,3, \cdots, n$ - observações

$u$ - número de incógnitas

Assim, quando a quantidade de observações $n$ é maior que a quantidade de incógnitas $u$, deve-se aplicar um processo de ajustamento pelo MMQ, para a determinação do melhor valor para as incógnitas.

Para a aplicação do MMQ, deve-se, inicialmente, estabelecer as equações de erros, que relaci- 
onam os valores medidos com os valores calculados pelas equações de observações, conforme indicado a seguir:

$$
\bar{L}_{i}=l_{i}+v_{i}=f_{i}\left(\bar{X}_{1}, \bar{X}_{2}, \bar{X}_{3}, \cdots, X_{u}\right)
$$

Sendo,

$\bar{L}_{i}$ - observações ajustadas

$v_{i}$ - erros residuais

$\bar{X}_{1}, \bar{X}_{2}, \bar{X}_{3}, \cdots, \bar{X}_{u^{-}}$Valores compensados das incógnitas.

De acordo com a equação (4.4), cada observação compensada é expressa como uma função, linear ou não, dos valores compensados das incógnitas, os quais são expressos como uma função de valores provisórios, adotados para cada incógnita. Tem-se, assim,

$$
\bar{X}_{k}=X_{k_{0}}+\delta X_{k}
$$

Sendo,

$k=1,2,3, \cdots, u$

$X_{k_{0}}$ - valor provisório da incógnita

$\delta X_{k}$ - acréscimo desconhecido

No caso da função $f$ não ser linear, ela deve ser linearizada, conforme indicado a seguir:

$$
\begin{gathered}
f_{i}\left(\bar{X}_{1}, \bar{X}_{2}, \bar{X}_{3}, \cdots, \bar{X}_{u}\right)=f_{i}\left(\bar{X}_{1}^{0}, \bar{X}_{2}^{0}, \bar{X}_{3}^{0}, \cdots, \bar{X}_{u}^{0}\right)+ \\
+\left(\frac{\partial f_{i}}{\partial X_{1}}\right)^{0} \cdot \delta X_{1}+\left(\frac{\partial f_{i}}{\partial X_{2}}\right)^{0} \cdot \delta X_{2}+\left(\frac{\partial f_{i}}{\partial X_{3}}\right)^{0} \cdot \delta X_{3}+\cdots+\left(\frac{\partial f_{i}}{\partial X_{n}}\right)^{0} \cdot \delta X_{n}
\end{gathered}
$$

Considerando,

$$
\begin{gathered}
a_{i}=\left(\frac{\partial f_{i}}{\partial X_{1}}\right)^{0} \cdot \delta X_{1}, \quad b_{i}=\left(\frac{\partial f_{i}}{\partial X_{2}}\right)^{0} \cdot \delta X_{2}, \quad c_{i}=\left(\frac{\partial f_{i}}{\partial X_{3}}\right)^{0} \cdot \delta X_{3}, \cdots, n_{i}=\left(\frac{\partial f_{i}}{\partial X_{n}}\right)^{0} \cdot \delta X_{n} \\
L_{i}=l_{i}-f_{i}\left(X_{1}^{0}, X_{2}^{0}, X_{3}^{0}, \cdots, X_{u}^{0}\right)
\end{gathered}
$$

Obtêm-se as equações de erros linearizadas descritas pela equação (4.9).

$$
v_{i}=a_{i} \cdot \delta X_{1}+b_{i} \cdot \delta X_{2}+c_{i} \cdot \delta X_{3}+\cdots-L_{i}
$$


Para simplificar o desenvolvimento matemático, consideram-se as incógnitas $(X, Y, Z)$, com a realização de $n$ observações. Tem-se, assim, o seguinte sistema de equações de erros:

$$
\begin{aligned}
& v_{1}=a_{1} \cdot \delta X+b_{1} \cdot \delta Y+c_{1} \cdot \delta Z-L_{1} \\
& v_{2}=a_{2} \cdot \delta X+b_{2} \cdot \delta Y+c_{2} \cdot \delta Z-L_{2} \\
& v_{3}=a_{3} \cdot \delta X+b_{3} \cdot \delta Y+c_{3} \cdot \delta Z-L_{3} \\
& v_{n}=a_{n} \cdot \delta X+b_{n} \cdot \delta Y+c_{n} \cdot \delta Z-L_{n}
\end{aligned}
$$

Que podem ser escritas na forma de matriz, como segue:

$$
V=\left[\begin{array}{c}
v_{1} \\
v_{2} \\
v_{3} \\
\vdots \\
v_{n}
\end{array}\right] \quad A=\left[\begin{array}{ccccc}
a_{11} & b_{12} & c_{13} & \cdots & n_{1 n} \\
a_{21} & b_{22} & c_{23} & \cdots & n_{2 n} \\
a_{31} & b_{32} & c_{32} & \cdots & n_{3 n} \\
\vdots & \vdots & \vdots & \ddots & \vdots \\
a_{n 1} & b_{n 2} & c_{n 3} & \cdots & n_{n n}
\end{array}\right] \quad X=\left[\begin{array}{c}
X \\
Y \\
Z
\end{array}\right] \quad L=\left[\begin{array}{c}
L_{1} \\
L_{2} \\
L_{3} \\
\vdots \\
L_{n}
\end{array}\right]
$$

Caso as observações não sejam todas de mesma precisão, devem-se considerar também os pesos de cada observação, a partir da matriz de pesos, conforme indicado a seguir:

$$
P=\left[\begin{array}{ccccc}
P_{1} & & & & \text { (zeros) } \\
& P_{2} & & & \\
& & P_{3} & & \\
& & & \ddots & \\
(\text { zeros }) & & & & P_{n}
\end{array}\right]
$$

Na forma de matriz, tem-se a seguinte equação matricial para as equações de erros:

$$
V=A X-L
$$

Com,

$$
\bar{X}=X^{0}+X
$$

Sendo,

$X^{0}$ - valores provisórios 
$\bar{X}$ - vetor das incógnitas compensadas

$V$ - vetor dos resíduos

$A$ - matriz das derivadas parciais

$X$ - vetor correção dos valores dos valores provisórios

$L$ - vetor dos valores observados, sendo $L=\left(l^{O}-l^{C}\right)$

$l^{O}$ - vetor das observações

$l^{C}$ - vetor dos parâmetros calculados ou em função dos parâmetros aproximados

$P$ - vetor dos pesos

$g l$ - grau de liberdade $(g l=n-u)$

$\sigma$ - desvio padrão

$\sigma^{2}$ - variância

$\hat{\sigma}^{2}$ - variância estimada

$\sigma_{0}^{2}$ - variância da observação de peso unitário a a priori

$\hat{\sigma}_{0}^{2}$ - variância da observação de peso unitário a posteriori

O princípio dos mínimos quadrados consiste em minimizar a relação $\left(V^{T} P V\right)$. Assim,

$$
V^{T} P V=\left(X^{T} A^{T}-L^{T}\right) P(A X-L)=X^{T} A^{T} P A X-X^{T} A^{T} P L-L^{T} P A X+L^{T} P L
$$

Como,

$$
X^{T} A^{T} P L=L^{T} P A X
$$

Tem-se:

$$
V^{T} P V=X^{T} A^{T} P A X-2 L^{T} P A X+L^{T} P L
$$

A função acima possui o seu extremo quando a sua derivada é nula. Dessa forma,

$$
\frac{\partial V^{T} P V}{\partial X}=2 X^{T} A^{T} P A-2 L^{T} P A X=0
$$

De onde, obtém-se:

$$
\begin{aligned}
& A^{T} P A X-A^{T} P L=0 \\
& X=\left(A^{T} P A\right)^{-1}\left(A^{T} P L\right)
\end{aligned}
$$


Em seguida, pode-se calcular o erro médio quadrático ou variância $\hat{\sigma}_{0}^{2}$ a posteriori da observação padrão de peso igual a 1, de acordo com a equação (4.21).

$$
\hat{\sigma}_{0}^{2}=\frac{V^{T} P V}{n-u}
$$

Após o cálculo da matriz das incógnitas $X$, pode-se ainda explorar os resultados de maneira a obter todas as informações estatísticas do modelo, essencialmente, as contidas nas matrizes de variância-covariância das incógnitas. Dessa forma,

Sabe-se que,

$$
K_{x x}=\hat{\sigma}_{0}^{2} \cdot\left(A^{T} P A\right)^{-1}
$$

Sendo,

$$
K_{x x}=\left[\begin{array}{ccccc}
\hat{\sigma}_{1}^{2} & \hat{\sigma}_{12} & \hat{\sigma}_{13} & \cdots & \hat{\sigma}_{1 n} \\
\hat{\sigma}_{21} & \hat{\sigma}_{2}^{2} & \hat{\sigma}_{23} & \cdots & \hat{\sigma}_{2 n} \\
\hat{\sigma}_{31} & \hat{\sigma}_{32} & \hat{\sigma}_{3}^{2} & \cdots & \hat{\sigma}_{3 n} \\
\vdots & \vdots & \vdots & \ddots & \vdots \\
\hat{\sigma}_{n 1} & \hat{\sigma}_{n 2} & \hat{\sigma}_{n 3} & \cdots & \hat{\sigma}_{n n}^{2}
\end{array}\right]
$$

Desta forma, tem-se,

$K_{x x}$ - Matriz variância-covariância das incógnitas

\subsection{Avaliação dos resultados}

Depois de determinadas, as coordenadas dos pontos de monitoramento precisam ser avaliadas para indicarem se houve ou não deformação ou deslocamento da estrutura monitorada. A este respeito, existem diferentes modelos matemáticos que podem ser aplicados, em função do tipo de monitoramento realizado (OGUNDARE, 2012).

Conforme já destacado, a técnica de medição mais simples utilizada para o monitoramento geodésico é o método polar. Neste caso, as coordenadas de cada ponto de monitoramento são determinadas a partir, de apenas um conjunto de observações, sem redundância. A avaliação da deformação ou do deslocamento, neste caso, é feita, baseando-se em parâmetros relacionados aos resultados obtidos, tais como, magnitude e velocidade do deslocamento.

Para os casos em que o método de medição permite o estabelecimento de equações com redundância de dados, torna-se possível aplicar testes estatísticos, para a verificação da confiabilidade dos resultados. Existem, para este propósito, vários testes estatísticos que podem ser aplicados. Os mais comuns de serem aplicados são o teste qui-quadrado e a elipse de erros, os quais estão brevemente apresentados a seguir. 


\subsubsection{Teste qui-quadrado}

Ao se tratar de controle de qualidade das observações e ajustamento, automaticamente deparase com modelos e testes estatísticos, mais especificamente com o Teste Global do Ajustamento ou, somente, Modelo Global. E quando este se baseia na distribuição $\chi^{2}$, o mesmo é conhecido por teste qui-quadrado. Este teste faz a comparação entre a variância $a$ priori $\sigma_{0}^{2}$ e a variância $a$ posteriori $\hat{\sigma}_{0}^{2}$ e, obviamente, ele somente tem significado, quando a variância a priori é conhecida. Porém, pode ser arbitrada pelo calculista, pois, a escolha de $\sigma_{0}^{2}$ não influencia no vetor das incógnitas (GHILANI, 2017). Desta maneira, propõe-se adotar $\sigma_{0}^{2}=0,001$. Já a $\hat{\sigma}_{0}^{2}$ é uma estimativa imparcial da $\sigma_{0}^{2}$ e é obtida após o ajustamento das observações, a partir da matriz dos resíduos $V$, dada pela equação (4.24):

$$
\hat{\sigma}_{0}^{2}=\frac{V^{T} P V}{g l}
$$

Sendo,

$V$ - matriz dos resíduos

$P$ - matriz peso das observações

$g l$ - grau de liberdade, obtido pela diferença entre o número de equações $n$ e o número de incógnitas $u$ a ser determinado, sendo, $g l=n-u$

Assim, ao considerar a distribuição $\chi^{2}$, o teste bilateral preconiza como hipótese básica nula, a seguinte relação (4.25):

$$
H_{0}: \hat{\sigma}_{0}^{2}=\sigma_{0}^{2} \quad \text { ou seja } \quad E\left\{\hat{\sigma}_{0}^{2} \mid H_{0}\right\}=\sigma_{0}^{2}
$$

Contra a hipótese alternativa, dada pela equação (4.26):

$$
H_{1}: \hat{\sigma}_{0}^{2} \neq \sigma_{0}^{2}
$$

Tem-se como estatística de comparação a equação (4.27):

$$
\chi^{* 2}(g l)=\frac{\hat{\sigma}_{0}^{2}}{\sigma_{0}^{2}} \cdot g l=\frac{V^{T} P V}{g l}
$$

Com os valores teóricos, dado pela equação (4.28):

$$
\chi_{r, \frac{\alpha}{2}}^{2} \quad \text { e } \quad \chi_{r, 1-\frac{\alpha}{2}}^{2}
$$


A hipótese básica não é rejeitada, ao nível de significância $\alpha$, se atender à condição imposta por (4.29):

$$
\chi^{* 2}<\chi_{r, 1-\frac{\alpha}{2}}^{2} \quad \text { ou } \quad \chi^{* 2}>\chi_{r, \frac{\alpha}{2}}^{2}
$$

Sendo,

$\chi^{* 2}$ - valor calculado para o teste qui-quadrado, equação (4.27)

$\chi^{2}$ - valor obtido da tabela de distribuição qui-quadrado

$\alpha$ - $\mathrm{n}$ de significância multidimensional

Caso contrário, deve-se proceder a uma análise cuidadosa do ajustamento, assim, pode haver erro na Matriz Variância Covariância (MVC) dos valores observados, ou os resíduos podem estar excessivamente grandes em decorrência de um erro grosseiro ou de erros sistemáticos. Ainda, o modelo matemático pode não ser consistente com as observações ou o sistema, como um todo, ser mal condicionado.

\subsubsection{Elipse de erro}

Após realizar o ajustamento das observações, obtém-se a matriz de variância-covariância dos parâmetros ajustados, sendo que, em sua diagonal principal, encontram-se as variâncias das coordenadas ajustadas. As raízes dessas variâncias fornecem o erro médio ou a precisão $\sigma_{x} \mathrm{e}$ $\sigma_{y}$ das coordenadas ajustadas, nos eixos $X$ e $Y$ do sistema de coordenadas utilizado. Ocorre, entretanto, que essas precisões indicam apenas as estimativas dos erros padrões nas direções dos eixos $X$ e $Y$, as quais podem não ser as direções dos erros máximos. A teoria estatística mostra que os erros em torno do ponto central estão distribuídos, segundo uma elipse de erros com inclinação $\theta$, semieixo menor $p$ e semieixo maior $q$ (GHILANI, 2017). Mostra-se que:

$$
\begin{gathered}
\operatorname{tg}(2 \theta)=\frac{2 Q_{x y}}{Q_{y y}-Q_{x x}} \\
p=\left(Q_{y y}-Q_{x y} \cdot \operatorname{cotg}(\theta)\right) \cdot \sigma_{0}^{2} \\
p=\left(Q_{x x}+Q_{x y} \cdot \operatorname{cotg}(\theta)\right) \cdot \sigma_{0}^{2}
\end{gathered}
$$

Sendo,

$\theta$ - ângulo de inclinação dos eixos da elipse em relação aos eixos $X$ e $Y$

$p$ - semieixo menor da elipse

$q$ - semieixo maior da elipse

$Q_{x y}$ - cofator $x y$ das coordenadas ajustadas 
$Q_{y y}$ - cofator $y$ das coordenadas ajustadas

$Q_{x x}$ - cofator $x$ das coordenadas ajustadas

A elipse de erros assim definida $1 \sigma$ indica que $38 \%$ dos desvios estão dentro da elipse considerada. Geralmente, para o monitoramento de estruturas, considera-se uma elipse com dimensões $1,96 \sigma$, ou seja, $95 \%$ dos desvios estão dentro da elipse considerada. 


\section{Capítulo}

\section{TÉCNICAS DE MEDIÇÕES ANGULARES E LINEARES PARA A REDE TOPOGRÁFICA ESPACIAL}

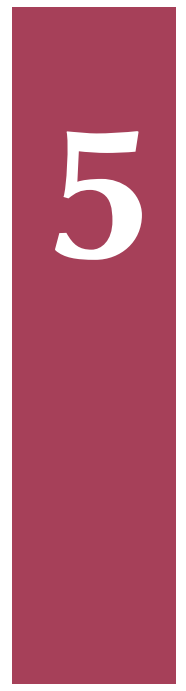

Com o advento das estações totais robóticas tornou-se possível realizar medições de direções horizontais, de ângulos verticais e de distâncias inclinadas, de forma cíclica e contínua. Ao mesmo tempo, os dados de observações passaram a ser coletados em três dimensões. Dessa forma, torna-se necessário desenvolver modelos de determinações e de ajustamento de coordenadas espaciais para se obter soluções $(3 D)$, de forma direta. Na sequência deste capítulo, serão apresentadas as modelagens matemáticas desenvolvidas e empregadas na realização da rede topográfica espacial proposta.

\subsection{Equação de observação para distância inclinada}

Para ajustar as observações de distâncias inclinadas, utilizando o método paramétrico dos mínimos quadrados, devem ser elaboradas as equações de observações que relacionam as quantidades observadas e seus erros aleatórios inerentes aos valores mais prováveis para as coordenadas $\left(X_{P}, Y_{P}, Z_{P}\right)$, que são os parâmetros das estações de referência envolvidas. Referindo-se à Figura 5.1, a equação (5.1), de distância inclinada, pode ser escrita para qualquer observação $d_{i P}^{\prime O}$, definida por:

$$
\begin{gathered}
d_{i P}^{\prime O}+v_{d_{i P}^{\prime O}}=d_{i P}^{\prime C} \\
d_{i P}^{\prime O}+v_{d_{i P}^{\prime O}}=\sqrt{\left(X_{P}-X_{i}\right)^{2}+\left(Y_{P}-Y_{i}\right)^{2}+\left(Z_{P}-Z_{i}\right)^{2}}
\end{gathered}
$$




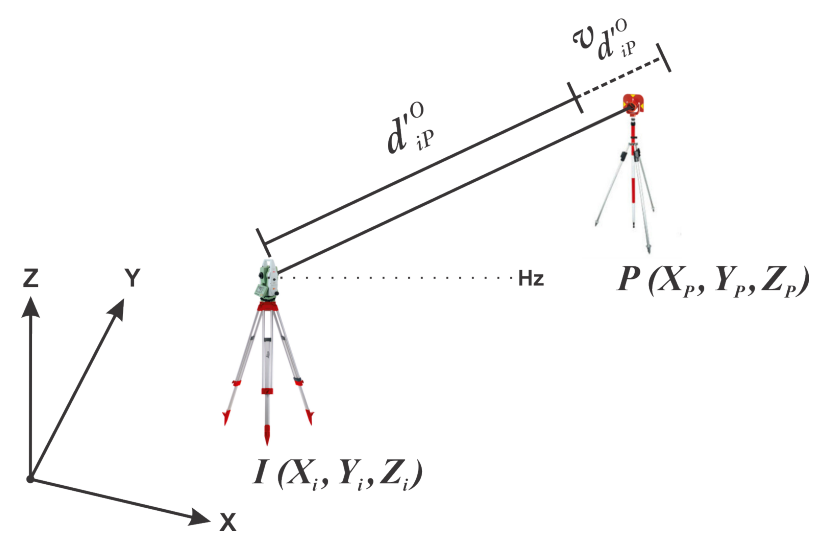

Figura 5.1: Observação de uma distância inclinada.

Na equação (5.1), $d_{i P}^{\prime O}$ é a distância inclinada observada de uma linha de visada entre as estações $I$ e $P, v_{d_{i P}^{\prime}}$ é o residual na observação $d_{i P}^{\prime O},\left(X_{i}, Y_{i}, Z_{i}\right)$ é o valor mais provável para a coordenada espacial da estação $I$ e $\left(X_{P}, Y_{P}, Z_{P}\right)$ é o valor mais provável para a coordenada espacial da estação $P$. A equação (5.1) é uma função não linear envolvendo as variáveis desconhecidas $\left(X_{i}, Y_{i}, Z_{i}\right)$ e $\left(X_{P}, Y_{P}, Z_{P}\right)$, e pode ser reescrita simplificadamente conforme indicado a seguir:

$$
F\left(X_{i}, Y_{i}, Z_{i}, X_{P}, Y_{P}, Z_{P}\right)=d_{i P}^{\prime O}+v_{d_{i P}^{\prime O}}
$$

Uma função não linear, como a equação (5.2), pode ser linearizada e resolvida, utilizando uma série de Taylor de primeira ordem de aproximação. Assim, tem-se:

$$
F\left(X_{i}^{0}, Y_{i}^{0}, Z_{i}^{0}, X_{P}^{0}, Y_{P}^{0}, Z_{P}^{0}\right)=F\left(P^{0}\right)
$$

Sendo,

$F$ - função a ser linearizada

$P^{0}$ - aproximação inicial

A forma linearizada da equação (5.2) é dada por:

$$
\begin{gathered}
F\left(X_{i}, Y_{i}, Z_{i}, X_{P}, Y_{P}, Z_{P}\right)=F\left(X_{i}^{0}, Y_{i}^{0}, Z_{i}^{0}, X_{P}^{0}, Y_{P}^{0}, Z_{P}^{0}\right)+ \\
+\frac{\partial F\left(P^{0}\right)}{\partial X_{i}} \delta X_{i}+\frac{\partial F\left(P^{0}\right)}{\partial Y_{i}} \delta Y_{i}+\frac{\partial F\left(P^{0}\right)}{\partial Z_{i}} \delta Z_{i}+ \\
+\frac{\partial F\left(P^{0}\right)}{\partial X_{P}} \delta X_{P}+\frac{\partial F\left(P^{0}\right)}{\partial Y_{P}} \delta Y_{P}+\frac{\partial F\left(P^{0}\right)}{\partial Z_{P}} \delta Z_{P}
\end{gathered}
$$


Onde $\frac{\partial F\left(P^{0}\right)}{\partial X_{i}} ; \frac{\partial F\left(P^{0}\right)}{\partial Y_{i}} ; \frac{\partial F\left(P^{0}\right)}{\partial Z_{i}} ; \frac{\partial F\left(P^{0}\right)}{\partial X_{P}} ; \frac{\partial F\left(P^{0}\right)}{\partial Y_{P}} ; \frac{\partial F\left(P^{0}\right)}{\partial Z_{P}}$ são as derivadas parciais de $F$ em relação a $\left(X_{i}, Y_{i}, Z_{i}\right)$ e $\left(X_{P}, Y_{P}, Z_{P}\right)$, respectivamente, avaliadas com os valores de coordenadas espaciais aproximados $\left(X_{i}^{0}, Y_{i}^{0}, Z_{i}^{0}\right)$ e $\left(X_{P}^{0}, Y_{P}^{0}, Z_{P}^{0}\right)$. As coordenadas espaciais $\left(X_{i}, Y_{i}, Z_{i}\right)$ e $\left(X_{P}, Y_{P}, Z_{P}\right)$ são os parâmetros desconhecidos, $\left(\delta X_{i}, \delta Y_{i}, \delta Z_{i}\right)$ e $\left(\delta X_{P}, \delta Y_{P}, \delta Z_{P}\right)$ são as correções para os valores de coordenadas de aproximação, tais que:

$$
\begin{array}{rrr}
X_{i}=X_{i}^{0}+\delta X_{i} & Y_{i}=Y_{i}^{0}+\delta Y_{i} & Z_{i}=Z_{i}^{0}+\delta Z_{i} \\
X_{P}=X_{P}^{0}+\delta X_{P} & Y_{P}=Y_{P}^{0}+\delta Y_{P} & Z_{P}=Z_{P}^{0}+\delta Z_{P}
\end{array}
$$

O cálculo de derivadas parciais é direto e será exemplificado como $\partial F / \partial X_{i}$.

$$
F\left(X_{i}, Y_{i}, Z_{i}, X_{P}, Y_{P}, Z_{P}\right)=\left[\left(X_{P}-X_{i}\right)^{2}+\left(Y_{P}-Y_{i}\right)^{2}+\left(Z_{P}-Z_{i}\right)^{2}\right]^{1 / 2}
$$

Ao se tomar a derivada da equação (5.5), em relação ao parâmetro $X_{i}$, tem-se:

$$
\frac{\partial F}{\partial X_{i}}=\frac{1}{2}\left[\left(X_{P}-X_{i}\right)^{2}+\left(Y_{P}-Y_{i}\right)^{2}+\left(Z_{P}-Z_{i}\right)^{2}\right]^{-1 / 2}\left[2\left(X_{P}-X_{i}\right)(-1)\right]
$$

Simplificando a equação (5.6), tem-se:

$$
\frac{\partial F}{\partial X_{i}}=\frac{-\left(X_{P}-X_{i}\right)}{\sqrt{\left(X_{P}-X_{i}\right)^{2}+\left(Y_{P}-Y_{i}\right)^{2}+\left(Z_{P}-Z_{i}\right)^{2}}}=\frac{X_{i}-X_{P}}{d_{i P}^{\prime C}}
$$

Com o emprego do mesmo procedimento, ou de forma análoga, obtém-se as derivadas parciais remanescentes:

$$
\begin{array}{lll}
\frac{\partial F}{\partial X_{i}}=\frac{X_{i}-X_{P}}{d_{i P}^{\prime C}} & \frac{\partial F}{\partial Y_{i}}=\frac{Y_{i}-Y_{P}}{d_{i P}^{\prime C}} & \frac{\partial F}{\partial Z_{i}}=\frac{Z_{i}-Z_{P}}{d_{i P}^{\prime C}} \\
\frac{\partial F}{\partial X_{P}}=\frac{X_{P}-X_{i}}{d_{i P}^{\prime C}} & \frac{\partial F}{\partial Y_{P}}=\frac{Y_{P}-Y_{i}}{d_{i P}^{\prime C}} & \frac{\partial F}{\partial Z_{P}}=\frac{Z_{P}-Z_{i}}{d_{i P}^{\prime C}}
\end{array}
$$

Se as equações (5.7) e (5.8) forem substituídas na equação (5.3) e os resultados substituídos pela equação (5.2), obtém-se a seguinte equação de observação linearizada, para distância inclinada:

$$
\begin{aligned}
L_{d_{i P}^{\prime}}+v_{d_{i P}^{\prime O}}= & \frac{X_{i}-X_{P}}{d_{i P^{0}}^{\prime C}} \delta X_{i}+\frac{Y_{i}-Y_{P}}{d_{i P^{0}}^{\prime C}} \delta Y_{i}+\frac{Z_{i}-Z_{P}}{d_{i P^{0}}^{\prime C}} \delta Z_{i}+ \\
& +\frac{X_{P}-X_{i}}{d_{i P^{0}}^{\prime C}} \delta X_{P}+\frac{Y_{P}-Y_{i}}{d_{i P^{0}}^{\prime C}} \delta Y_{P}+\frac{Z_{P}-Z_{i}}{d_{i P^{0}}^{\prime C}} \delta Z_{P}
\end{aligned}
$$

Onde $F\left(P^{0}\right)$ é calculado nos valores aproximados dos parâmetros. Dessa forma, é possível compor a Matriz $L$ relativa às diferenças de distâncias inclinadas observadas e calculadas, como segue, $L_{d_{i P}^{\prime}}=d_{i P}^{\prime O}-d_{i P^{0}}^{\prime C}$. 
Sendo,

$$
\begin{gathered}
d_{i P^{0}}^{\prime C}=F\left(X_{i}^{0}, Y_{i}^{0}, Z_{i}^{0}, X_{P}^{0}, Y_{P}^{0}, Z_{P}^{0}\right)=\sqrt{\left(X_{P}^{0}-X_{i}^{0}\right)^{2}+\left(Y_{P}^{0}-Y_{i}^{0}\right)^{2}+\left(Z_{P}^{0}-Z_{i}^{0}\right)^{2}} \\
d_{i P}^{C C}=F\left(X_{i}^{0}, Y_{i}^{0}, Z_{i}^{0}, X_{P}^{0}, Y_{P}^{0}, Z_{P}^{0}\right)+L_{d_{i P}^{\prime}}\left(P^{0}\right)
\end{gathered}
$$

Para compor a Matriz Jacobiana $A$ das distâncias inclinadas, dos ângulos verticais zenitais, dos ângulos verticais de altura e dos ângulos horizontais azimutais, foram considerados, nesta tese, $i$ como sendo o valor de $\partial F / \partial X_{i}$ e $P$ como sendo o valor de $\partial F / \partial X_{P}$ em seus respectivos pontos. Sendo assim, a estrutura da Matriz $A$ para distância inclinada da rede topográfica espacial é representada pela Tabela 5.1 .

\begin{tabular}{|c|c|c|c|c|c|c|c|c|c|c|c|c|c|c|c|}
\hline \multirow{2}{*}{$\begin{array}{c}\text { Distância inclinada } \\
\text { Observações (iP) }\end{array}$} & \multicolumn{15}{|c|}{ Incógnitas } \\
\hline & $\delta X_{2}$ & $\delta Y_{2}$ & $\delta Z_{2}$ & $\delta X_{3}$ & $\delta Y_{3}$ & $\delta Z_{3}$ & $\delta X_{4}$ & $\delta Y_{4}$ & $\delta Z_{4}$ & $\delta X_{5}$ & $\delta Y_{5}$ & $\delta Z_{5}$ & $\delta X_{6}$ & $\delta Y_{6}$ & $\delta Z_{6}$ \\
\hline $1-2$ & $P$ & $P$ & $P$ & & & & & & & & & & & & \\
\hline $1-3$ & & & & $P$ & $P$ & $P$ & & & & & & & & & \\
\hline $1-4$ & & & & & & & $P$ & $P$ & $P$ & & & & & & \\
\hline $1-5$ & & & & & & & & & & $P$ & $P$ & $P$ & & & \\
\hline $1-6$ & & & & & & & & & & & & & $P$ & $P$ & $P$ \\
\hline $2-3$ & $i$ & $i$ & $i$ & $P$ & $P$ & $P$ & & & & & & & & & \\
\hline $2-4$ & $i$ & $i$ & $i$ & & & & $P$ & $P$ & $P$ & & & & & & \\
\hline $2-5$ & $i$ & $i$ & $i$ & & & & & & & $P$ & $P$ & $P$ & & & \\
\hline $2-6$ & $i$ & $i$ & $i$ & & & & & & & & & & $P$ & $P$ & $P$ \\
\hline $2-1$ & $i$ & $i$ & $i$ & & & & & & & & & & & & \\
\hline $3-4$ & & & & $i$ & $i$ & $i$ & $P$ & $P$ & $P$ & & & & & & \\
\hline $3-5$ & & & & $i$ & $i$ & $i$ & & & & $P$ & $P$ & $P$ & & & \\
\hline $3-6$ & & & & $i$ & $i$ & $i$ & & & & & & & $P$ & $P$ & $P$ \\
\hline $3-1$ & & & & $i$ & $i$ & $i$ & & & & & & & & & \\
\hline $3-2$ & $P$ & $P$ & $P$ & $i$ & $i$ & $i$ & & & & & & & & & \\
\hline $4-5$ & & & & & & & $i$ & $i$ & $i$ & $P$ & $P$ & $P$ & & & \\
\hline $4-6$ & & & & & & & $i$ & $i$ & $i$ & & & & $P$ & $P$ & $P$ \\
\hline $4-1$ & & & & & & & $i$ & $i$ & $i$ & & & & & & \\
\hline $4-2$ & $P$ & $P$ & $P$ & & & & $i$ & $i$ & $i$ & & & & & & \\
\hline $4-3$ & & & & $P$ & $P$ & $P$ & $i$ & $i$ & $i$ & & & & & & \\
\hline $5-6$ & & & & & & & & & & $i$ & $i$ & $i$ & $P$ & $P$ & $P$ \\
\hline 5-1 & & & & & & & & & & $i$ & $i$ & $i$ & & & \\
\hline $5-2$ & $P$ & $P$ & $P$ & & & & & & & $i$ & $i$ & $i$ & & & \\
\hline $5-3$ & & & & $P$ & $P$ & $P$ & & & & $i$ & $i$ & $i$ & & & \\
\hline $5-4$ & & & & & & & $P$ & $P$ & $P$ & $i$ & $i$ & $i$ & & & \\
\hline $6-1$ & & & & & & & & & & & & & $i$ & $i$ & $i$ \\
\hline $6-2$ & $P$ & $P$ & $P$ & & & & & & & & & & $i$ & $i$ & $i$ \\
\hline $6-3$ & & & & $P$ & $P$ & $P$ & & & & & & & $i$ & $i$ & $i$ \\
\hline $6-4$ & & & & & & & $P$ & $P$ & $P$ & & & & $i$ & $i$ & $i$ \\
\hline $6-5$ & & & & & & & & & & $P$ & $P$ & $P$ & $i$ & $i$ & $i$ \\
\hline
\end{tabular}

Tabela 5.1: Estrutura da Matriz A para distância inclinada. 


\subsection{Equação de observação para ângulo vertical zenital}

A rede topográfica espacial executada nesta tese considerou o ângulo vertical zenital, mas como pode ser visto na seção 5.3 , também foi desenvolvida a equação de observação para ângulo vertical de altura.

Dessa forma, para ajustar as observações de ângulos verticais zenitais, utilizando o método paramétrico dos mínimos quadrados, devem ser elaboradas as equações de observação que relacionam as quantidades observadas e seus erros aleatórios, inerentes aos valores mais prováveis para as coordenadas $\left(X_{P}, Y_{P}, Z_{P}\right)$, que são os parâmetros das estações envolvidas.

Como mostra a Figura 5.2, a equação (5.12), de ângulo vertical zenital, pode ser escrita para qualquer observação $z_{i P}^{O}$, definida por:

$$
\begin{gathered}
z_{i P}^{O}+v_{z_{i P}^{O}}=z_{i P}^{C} \\
z_{i P}^{O}+v_{z_{i P}^{O}}=\cos ^{-1} \frac{\left(\Delta Z_{i P}\right)}{d_{i P}^{\prime C}}=\cos ^{-1} \frac{\left(Z_{P}-Z_{i}\right)}{\sqrt{\left(X_{P}-X_{i}\right)^{2}+\left(Y_{P}-Y_{i}\right)^{2}+\left(Z_{P}-Z_{i}\right)^{2}}}
\end{gathered}
$$

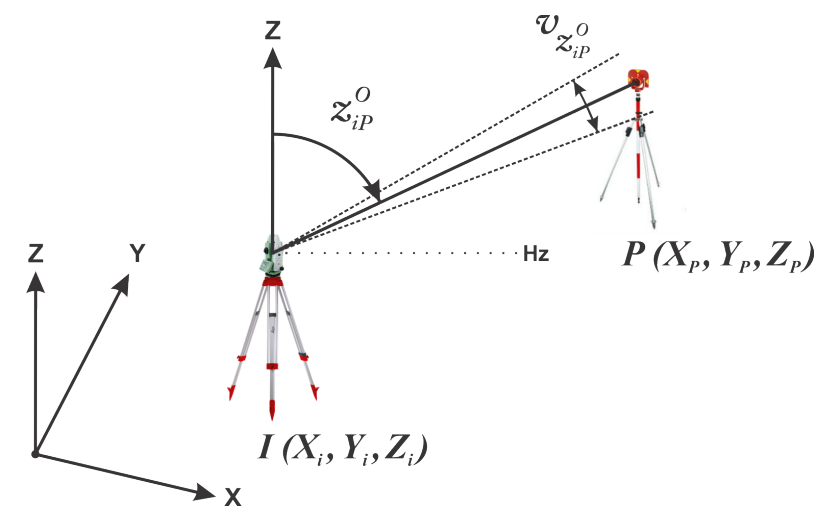

Figura 5.2: Observação de um ângulo vertical zenital.

Dessa maneira, a fórmula do ângulo vertical zenital, em função dos parâmetros $\left(X_{i}, Y_{i}, Z_{i}\right)$ e $\left(X_{P}, Y_{P}, Z_{P}\right)$, pode ser reescrita:

$$
F\left(X_{i}, Y_{i}, Z_{i}, X_{P}, Y_{P}, Z_{P}\right)=z_{i P}^{O}+v_{z_{i P}^{O}}
$$

Um sistema de equações não lineares, como a equação (5.13), pode ser linearizada e resolvida utilizando uma série de Taylor, de primeira ordem de aproximação. Sendo,

$$
F\left(X_{i}^{0}, Y_{i}^{0}, Z_{i}^{0}, X_{P}^{0}, Y_{P}^{0}, Z_{P}^{0}\right)=F\left(P^{0}\right)
$$


A forma linearizada da equação (5.13) é dada por:

$$
\begin{gathered}
F\left(X_{i}, Y_{i}, Z_{i}, X_{P}, Y_{P}, Z_{P}\right)=F\left(X_{i}^{0}, Y_{i}^{0}, Z_{i}^{0}, X_{P}^{0}, Y_{P}^{0}, Z_{P}^{0}\right)+ \\
+\frac{\partial F\left(P^{0}\right)}{\partial X_{i}} \delta X_{i}+\frac{\partial F\left(P^{0}\right)}{\partial Y_{i}} \delta Y_{i}+\frac{\partial F\left(P^{0}\right)}{\partial Z_{i}} \delta Z_{i}+ \\
+\frac{\partial F\left(P^{0}\right)}{\partial X_{P}} \delta X_{P}+\frac{\partial F\left(P^{0}\right)}{\partial Y_{P}} \delta Y_{P}+\frac{\partial F\left(P^{0}\right)}{\partial Z_{P}} \delta Z_{P}
\end{gathered}
$$

Onde $\frac{\partial F\left(P^{0}\right)}{\partial X_{i}} ; \frac{\partial F\left(P^{0}\right)}{\partial Y_{i}} ; \frac{\partial F\left(P^{0}\right)}{\partial Z_{i}} ; \frac{\partial F\left(P^{0}\right)}{\partial X_{P}} ; \frac{\partial F\left(P^{0}\right)}{\partial Y_{P}} ; \frac{\partial F\left(P^{0}\right)}{\partial Z_{P}}$ são as derivadas parciais de $F$ em relação a $\left(X_{i}, Y_{i}, Z_{i}\right)$ e $\left(X_{P}, Y_{P}, Z_{P}\right)$, respectivamente, avaliadas com os valores de coordenadas espaciais aproximados $\left(X_{i}^{0}, Y_{i}^{0}, Z_{i}^{0}\right)$ e $\left(X_{P}^{0}, Y_{P}^{0}, Z_{P}^{0}\right)$. As coordenadas espaciais $\left(X_{i}, Y_{i}, Z_{i}\right)$ e $\left(X_{P}, Y_{P}, Z_{P}\right)$ são os parâmetros desconhecidos, $\left(\delta X_{i}, \delta Y_{i}, \delta Z_{i}\right)$ e $\left(\delta X_{P}, \delta Y_{P}, \delta Z_{P}\right)$ são as correções para os valores de coordenadas de aproximação tais que:

$$
\begin{array}{rrr}
X_{i}=X_{i}^{0}+\delta X_{i} & Y_{i}=Y_{i}^{0}+\delta Y_{i} & Z_{i}=Z_{i}^{0}+\delta Z_{i} \\
X_{P}=X_{P}^{0}+\delta X_{P} & Y_{P}=Y_{P}^{0}+\delta Y_{P} & Z_{P}=Z_{P}^{0}+\delta Z_{P}
\end{array}
$$

Para determinar as derivadas parciais da equação (5.14), é necessário conhecer a derivada da função inversa de $\cos ^{-1}$ em relação à $x$, a qual se sabe que é:

$$
\frac{d}{d x} \cos ^{-1} u=\frac{-1}{\sqrt{1-u^{2}}} \frac{d u}{d x}
$$

Utilizando a equação (5.16), o procedimento para determinar a derivada parcial $\partial F / \partial X_{i}$, é demonstrado como segue:

$$
\frac{\partial F}{\partial X_{i}}=\frac{-1}{\sqrt{1-\frac{\left(Z_{P}-Z_{i}\right)^{2}}{d_{i P}^{\prime C}}}}\left[-\frac{1}{2}\left(Z_{P}-Z_{i}\right)\right]\left[\left(d_{i P}^{\prime C}\right)^{-3 / 2}\right]\left[2\left(X_{P}-X_{i}\right)(-1)\right]
$$

Simplificando a equação (5.17), tem-se:

$$
\frac{\partial F}{\partial X_{i}}=\left[\frac{-1}{\sqrt{1-\frac{\left(Z_{P}-Z_{i}\right)^{2}}{d_{i P}^{\prime C}}}}\right]\left[\left(X_{P}-X_{i}\right)\left(Z_{P}-Z_{i}\right)\right]\left[\frac{1}{\sqrt{\left(d_{i P}^{\prime C}\right)^{3}}}\right]
$$


Com o emprego do mesmo procedimento, ou de forma análoga, obtém-se as derivadas parciais remanescentes:

$$
\begin{aligned}
& \frac{\partial F}{\partial Y_{i}}=\left[\frac{-1}{\sqrt{1-\frac{\left(Z_{P}-Z_{i}\right)^{2}}{d_{i P}^{\prime C}}}}\right]\left[\left(Y_{P}-Y_{i}\right)\left(Z_{P}-Z_{i}\right)\right]\left[\frac{1}{\sqrt{\left(d_{i P}^{\prime C}\right)^{3}}}\right] \\
& \frac{\partial F}{\partial Z_{i}}=\left[\frac{-1}{\sqrt{1-\frac{\left(Z_{P}-Z_{i}\right)^{2}}{d_{i P}^{\prime C}}}}\right]\left[\left(Z_{P}-Z_{i}\right)^{2}\left(\frac{1}{\sqrt{\left(d_{i P}^{\prime C}\right)^{3}}}-\frac{1}{d_{i P}^{\prime C}}\right)\right]
\end{aligned}
$$

Na sequência, segue a derivada parcial em relação à coordenada $X_{P}$,

$$
\frac{\partial F}{\partial X_{P}}=\frac{-1}{\sqrt{1-\frac{\left(Z_{P}-Z_{i}\right)^{2}}{d_{i P}^{\prime C}}}}\left[-\frac{1}{2}\left(Z_{P}-Z_{i}\right)\right]\left[\left(d_{i P}^{\prime C}\right)^{-3 / 2}\right]\left[2\left(X_{P}-X_{i}\right)\right]
$$

Simplificando a equação (5.21), tem-se,

$$
\frac{\partial F}{\partial X_{P}}=\left[\frac{+1}{\sqrt{1-\frac{\left(Z_{P}-Z_{i}\right)^{2}}{d_{i P}^{\prime C}}}}\right]\left[\left(X_{P}-X_{i}\right)\left(Z_{P}-Z_{i}\right)\right]\left[\frac{1}{\sqrt{\left(d_{i P}^{\prime C}\right)^{3}}}\right]
$$

A derivada parcial em relação à coordenada $Y_{P}$ é dado como segue:

$$
\frac{\partial F}{\partial Y_{P}}=\left[\frac{+1}{\sqrt{1-\frac{\left(Z_{P}-Z_{i}\right)^{2}}{d_{i P}^{\prime}}}}\right]\left[\left(Y_{P}-Y_{i}\right)\left(Z_{P}-Z_{i}\right)\right]\left[\frac{1}{\sqrt{\left(d_{i P}^{\prime C}\right)^{3}}}\right]
$$

Por fim tem-se a derivada parcial em relação à coordenada $Z_{P}$,

$$
\frac{\partial F}{\partial Z_{P}}=\left[\frac{-1}{\sqrt{1-\frac{\left(Z_{P}-Z_{i}\right)^{2}}{d_{i P}^{\prime C}}}}\right]\left[\frac{1}{d_{i P}^{\prime C}}-\left(Z_{P}-Z_{i}\right)^{2}\left(\frac{1}{\sqrt{\left(d_{i P}^{\prime C}\right)^{3}}}\right)\right]
$$

Se as equações (5.18 a 5.24) forem substituídas pela equação (5.14) e os resultados substituídos pela equação (5.13), obtém-se a seguinte equação de observação linearizada para ângulo 
vertical zenital:

$$
\begin{aligned}
& L_{z_{i P}}+v_{z_{i P}^{O}}=\left[\frac{-1}{\sqrt{1-\frac{\left(Z_{P}-Z_{i}\right)^{2}}{d_{i P}^{\prime C}}}}\right]\left[\left(X_{P}-X_{i}\right)\left(Z_{P}-Z_{i}\right)\right]\left[\frac{1}{\sqrt{\left(d_{i P}^{\prime C}\right)^{3}}}\right] \delta X_{i}+ \\
& +\frac{-1}{\sqrt{1-\frac{\left(Z_{P}-Z_{i}\right)^{2}}{d_{i P}^{\prime}}}}\left[\left(Y_{P}-Y_{i}\right)\left(Z_{P}-Z_{i}\right)\right]\left[\frac{1}{\sqrt{\left(d_{i P}^{\prime C}\right)^{3}}}\right] \delta Y_{i}+ \\
& +\left[\frac{-1}{\sqrt{1-\frac{\left(Z_{P}-Z_{i}\right)^{2}}{d_{i P}^{\prime C}}}}\right]\left[\left(Z_{P}-Z_{i}\right)^{2}\left(\frac{1}{\sqrt{\left(d_{i P}^{\prime C}\right)^{3}}}-\frac{1}{d_{i P}^{\prime C}}\right)\right] \delta Z_{i}+ \\
& +\left[\frac{+1}{\sqrt{1-\frac{\left(Z_{P}-Z_{i}\right)^{2}}{d_{i P}^{\prime C}}}}\right]\left[\left(X_{P}-X_{i}\right)\left(Z_{P}-Z_{i}\right)\right]\left[\frac{1}{\sqrt{\left(d_{i P}^{\prime C}\right)^{3}}}\right] \delta X_{P}+ \\
& +\left[\frac{+1}{\sqrt{1-\frac{\left(Z_{P}-Z_{i}\right)^{2}}{d_{i P}^{\prime \prime}}}}\right]\left[\left(Y_{P}-Y_{i}\right)\left(Z_{P}-Z_{i}\right)\right]\left[\frac{1}{\sqrt{\left(d_{i P}^{\prime C}\right)^{3}}}\right] \delta Y_{P}+ \\
& +\left[\frac{-1}{\sqrt{1-\frac{\left(Z_{P}-Z_{i}\right)^{2}}{d_{i P}^{\prime C}}}}\right]\left[\frac{1}{d_{i P}^{\prime C}}-\left(Z_{P}-Z_{i}\right)^{2}\left(\frac{1}{\sqrt{\left(d_{i P}^{\prime C}\right)^{3}}}\right)\right] \delta Z_{P}
\end{aligned}
$$

Onde $F\left(P^{0}\right)$ é calculado nos valores aproximados dos parâmetros, $L_{z_{i P}}=z_{i P}^{O}-z_{i P^{0}}^{C}$, e

$$
\begin{gathered}
L_{z_{i P}}=z_{i P}^{O}-\left[\cos ^{-1} \frac{\left(\Delta Z_{i P^{0}}\right)}{\left(d_{i P^{0}}^{C}\right)}\right] \\
z_{i P}^{C}=F\left(X_{i}^{0}, Y_{i}^{0}, Z_{i}^{0}, X_{P}^{0}, Y_{P}^{0}, Z_{P}^{0}\right)+L_{z_{i P}}\left(P^{0}\right)
\end{gathered}
$$

Desta forma é possível compor a Matriz Jacobiana $A$, para as observações de ângulos verticais zenitais, cuja estrutura é representada pela Tabela 5.2. 
Tabela 5.2: Estrutura da Matriz A para ângulo vertical zenital.

\begin{tabular}{|c|c|c|c|c|c|c|c|c|c|c|c|c|c|c|c|}
\hline \multirow{2}{*}{$\begin{array}{c}\text { Ângulo vertical zenital } \\
\text { Observações (iP) }\end{array}$} & \multicolumn{15}{|c|}{ Incógnitas } \\
\hline & $\delta X_{2}$ & $\delta Y_{2}$ & $\delta Z_{2}$ & $\delta X_{3}$ & $\delta Y_{3}$ & $\delta Z_{3}$ & $\delta X_{4}$ & $\delta Y_{4}$ & $\delta Z_{4}$ & $\delta X_{5}$ & $\delta Y_{5}$ & $\delta Z_{5}$ & $\delta X_{6}$ & $\delta Y_{6}$ & $\delta Z_{c}$ \\
\hline $1-2$ & $P$ & $P$ & $P$ & & & & & & & & & & & & \\
\hline $1-3$ & & & & $P$ & $P$ & $P$ & & & & & & & & & \\
\hline $1-4$ & & & & & & & $P$ & $P$ & $P$ & & & & & & \\
\hline $1-5$ & & & & & & & & & & $P$ & $P$ & $P$ & & & \\
\hline $1-6$ & & & & & & & & & & & & & $P$ & $P$ & $P$ \\
\hline $2-3$ & $i$ & $i$ & $i$ & $P$ & $P$ & $P$ & & & & & & & & & \\
\hline $2-4$ & $i$ & $i$ & $i$ & & & & $P$ & $P$ & $P$ & & & & & & \\
\hline $2-5$ & $i$ & $i$ & $i$ & & & & & & & $P$ & $P$ & $P$ & & & \\
\hline $2-6$ & $i$ & $i$ & $i$ & & & & & & & & & & $P$ & $P$ & $P$ \\
\hline $2-1$ & $i$ & $i$ & $i$ & & & & & & & & & & & & \\
\hline $3-4$ & & & & $i$ & $i$ & $i$ & $P$ & $P$ & $P$ & & & & & & \\
\hline $3-5$ & & & & $i$ & $i$ & $i$ & & & & $P$ & $P$ & $P$ & & & \\
\hline $3-6$ & & & & $i$ & $i$ & $i$ & & & & & & & $P$ & $P$ & $P$ \\
\hline $3-1$ & & & & $i$ & $i$ & $i$ & & & & & & & & & \\
\hline $3-2$ & $P$ & $P$ & $P$ & $i$ & $i$ & $i$ & & & & & & & & & \\
\hline $4-5$ & & & & & & & $i$ & $i$ & $i$ & $P$ & $P$ & $P$ & & & \\
\hline $4-6$ & & & & & & & $i$ & $i$ & $i$ & & & & $P$ & $P$ & $P$ \\
\hline $4-1$ & & & & & & & $i$ & $i$ & $i$ & & & & & & \\
\hline $4-2$ & $P$ & $P$ & $P$ & & & & $i$ & $i$ & $i$ & & & & & & \\
\hline $4-3$ & & & & $P$ & $P$ & $P$ & $i$ & $i$ & $i$ & & & & & & \\
\hline $5-6$ & & & & & & & & & & $i$ & $i$ & $i$ & $P$ & $P$ & $P$ \\
\hline $5-1$ & & & & & & & & & & $i$ & $i$ & $i$ & & & \\
\hline $5-2$ & $P$ & $P$ & $P$ & & & & & & & $i$ & $i$ & $i$ & & & \\
\hline $5-3$ & & & & $P$ & $P$ & $P$ & & & & $i$ & $i$ & $i$ & & & \\
\hline $5-4$ & & & & & & & $P$ & $P$ & $P$ & $i$ & $i$ & $i$ & & & \\
\hline $6-1$ & & & & & & & & & & & & & $i$ & $i$ & $i$ \\
\hline $6-2$ & $P$ & $P$ & $P$ & & & & & & & & & & $i$ & $i$ & $i$ \\
\hline $6-3$ & & & & $P$ & $P$ & $P$ & & & & & & & $i$ & $i$ & $i$ \\
\hline $6-4$ & & & & & & & $P$ & $P$ & $P$ & & & & $i$ & $i$ & $i$ \\
\hline $6-5$ & & & & & & & & & & $P$ & $P$ & $P$ & $i$ & $i$ & $i$ \\
\hline
\end{tabular}

\subsection{Equação de observação para ângulo vertical de altura}

A rede topográfica espacial, pode também ser determinada com o uso de ângulos verticais de altura, a qual não foi aplicada nesta tese.

Assim, para ajustar as observações de ângulos verticais de altura, utilizando o método paramétrico dos mínimos quadrados, devem ser elaboradas as equações de observação que relacionam as quantidades observadas e seus erros aleatórios, inerentes aos valores mais prováveis para as coordenadas $\left(X_{P}, Y_{P}, Z_{P}\right)$, que são os parâmetros das estações envolvidas. Como pode ser visto na Figura 5.3, a equação (5.28), de ângulo vertical de altura, pode ser escrita para qualquer observação $\beta_{i P}^{O}$, definida por:

$$
\begin{gathered}
\beta_{i P}^{O}+v_{\beta_{i P}^{O}}=\beta_{i P}^{C} \\
\beta_{i P}^{O}+v_{\beta_{i P}^{O}}=\operatorname{sen}^{-1} \frac{\left(\Delta Z_{i P}\right)}{d_{i P}^{\prime C}}=\operatorname{sen}^{-1} \frac{\left(Z_{P}-Z_{i}\right)}{\sqrt{\left(X_{P}-X_{i}\right)^{2}+\left(Y_{P}-Y_{i}\right)^{2}+\left(Z_{P}-Z_{i}\right)^{2}}}
\end{gathered}
$$




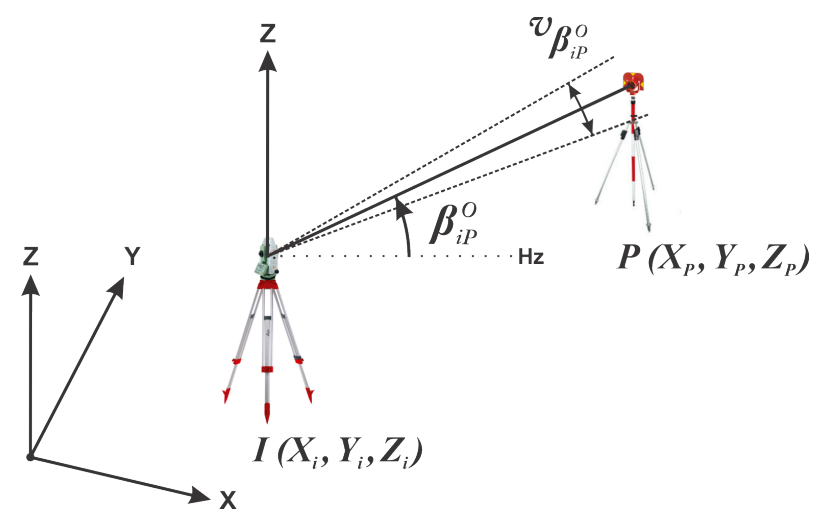

Figura 5.3: Observação de um ângulo vertical de altura.

Desta maneira, a fórmula do ângulo vertical de altura, em função dos parâmetros $\left(X_{i}, Y_{i}, Z_{i}\right)$ e $\left(X_{P}, Y_{P}, Z_{P}\right)$, pode ser reescrita:

$$
F\left(X_{i}, Y_{i}, Z_{i}, X_{P}, Y_{P}, Z_{P}\right)=\beta_{i P}^{O}+v_{\beta_{i P}^{O}}
$$

Um sistema de equações não lineares, como a equação (5.29), pode ser linearizada e resolvida, utilizando uma série de Taylor de primeira ordem de aproximação. Sendo,

$$
F\left(X_{i}^{0}, Y_{i}^{0}, Z_{i}^{0}, X_{P}^{0}, Y_{P}^{0}, Z_{P}^{0}\right)=F\left(P^{0}\right)
$$

A forma linearizada da equação (5.29) é dada por:

$$
\begin{gathered}
F\left(X_{i}, Y_{i}, Z_{i}, X_{P}, Y_{P}, Z_{P}\right)=F\left(X_{i}^{0}, Y_{i}^{0}, Z_{i}^{0}, X_{P}^{0}, Y_{P}^{0}, Z_{P}^{0}\right)+ \\
+\frac{\partial F\left(P^{0}\right)}{\partial X_{i}} \delta X_{i}+\frac{\partial F\left(P^{0}\right)}{\partial Y_{i}} \delta Y_{i}+\frac{\partial F\left(P^{0}\right)}{\partial Z_{i}} \delta Z_{i}+ \\
+\frac{\partial F\left(P^{0}\right)}{\partial X_{P}} \delta X_{P}+\frac{\partial F\left(P^{0}\right)}{\partial Y_{P}} \delta Y_{P}+\frac{\partial F\left(P^{0}\right)}{\partial Z_{P}} \delta Z_{P}
\end{gathered}
$$

Onde $\frac{\partial F\left(P^{0}\right)}{\partial X_{i}} ; \frac{\partial F\left(P^{0}\right)}{\partial Y_{i}} ; \frac{\partial F\left(P^{0}\right)}{\partial Z_{i}} ; \frac{\partial F\left(P^{0}\right)}{\partial X_{P}} ; \frac{\partial F\left(P^{0}\right)}{\partial Y_{P}} ; \frac{\partial F\left(P^{0}\right)}{\partial Z_{P}}$ são as derivadas parciais de $F$ em relação a $\left(X_{i}, Y_{i}, Z_{i}\right)$ e $\left(X_{P}, Y_{P}, Z_{P}\right)$, respectivamente, avaliadas com os valores de coordenadas espaciais aproximados $\left(X_{i}^{0}, Y_{i}^{0}, Z_{i}^{0}\right)$ e $\left(X_{P}^{0}, Y_{P}^{0}, Z_{P}^{0}\right)$. As coordenadas espaciais $\left(X_{i}, Y_{i}, Z_{i}\right)$ e $\left(X_{P}, Y_{P}, Z_{P}\right)$ são parâmetros desconhecidos, $\left(\delta X_{i}, \delta Y_{i}, \delta Z_{i}\right)$ e $\left(\delta X_{P}, \delta Y_{P}, \delta Z_{P}\right)$ são as correções para os valores de coordenadas de aproximação, tais que:

$$
\begin{array}{rrr}
X_{i}=X_{i}^{0}+\delta X_{i} & Y_{i}=Y_{i}^{0}+\delta Y_{i} & Z_{i}=Z_{i}^{0}+\delta Z_{i} \\
X_{P}=X_{P}^{0}+\delta X_{P} & Y_{P}=Y_{P}^{0}+\delta Y_{P} & Z_{P}=Z_{P}^{0}+\delta Z_{P}
\end{array}
$$


Para determinar as derivadas parciais da equação (5.30), é necessário conhecer a derivada da função inversa de $\operatorname{sen}^{-1}$ em relação à $x$, a qual se sabe que é:

$$
\frac{d}{d x} \operatorname{sen}^{-1} u=\frac{1}{\sqrt{1-u^{2}}} \frac{d u}{d x}
$$

Utilizando a equação (5.32), o procedimento para determinar a derivada parcial $\partial F / \partial X_{i}$ é demonstrado como segue:

$$
\frac{\partial F}{\partial X_{i}}=\frac{1}{\sqrt{1-\frac{\left(Z_{P}-Z_{i}\right)^{2}}{d_{i P}^{\prime C}}}}\left[-\frac{1}{2}\left(Z_{P}-Z_{i}\right)\right]\left[\left(d_{i P}^{\prime C}\right)^{-3 / 2}\right]\left[2\left(X_{P}-X_{i}\right)(-1)\right]
$$

Simplificando a equação (5.33), tem-se:

$$
\frac{\partial F}{\partial X_{i}}=\left[\frac{1}{\sqrt{1-\frac{\left(Z_{P}-Z_{i}\right)^{2}}{d_{i P}^{\prime C}}}}\right]\left[\left(X_{P}-X_{i}\right)\left(Z_{P}-Z_{i}\right)\right]\left[\frac{1}{\sqrt{\left(d_{i P}^{\prime C}\right)^{3}}}\right]
$$

Com emprego do mesmo procedimento, ou de forma análoga, obtém-se as derivadas parciais remanescentes:

$$
\begin{aligned}
& \frac{\partial F}{\partial Y_{i}}=\left[\frac{1}{\sqrt{1-\frac{\left(Z_{P}-Z_{i}\right)^{2}}{d_{i P}^{\prime C}}}}\right]\left[\left(Y_{P}-Y_{i}\right)\left(Z_{P}-Z_{i}\right)\right]\left[\frac{1}{\sqrt{\left(d_{i P}^{\prime C}\right)^{3}}}\right] \\
& \frac{\partial F}{\partial Z_{i}}=\left[\frac{1}{\sqrt{1-\frac{\left(Z_{P}-Z_{i}\right)^{2}}{d_{i P}^{\prime C}}}}\right]\left[\left(Z_{P}-Z_{i}\right)^{2}\left(\frac{1}{\sqrt{\left(d_{i P}^{\prime C}\right)^{3}}}-\frac{1}{d_{i P}^{\prime C}}\right)\right]
\end{aligned}
$$

Na sequência, segue a derivada parcial em relação à coordenada $X_{P}$,

$$
\frac{\partial F}{\partial X_{P}}=\frac{1}{\sqrt{1-\frac{\left(Z_{P}-Z_{i}\right)^{2}}{d_{i P}^{\prime C}}}}\left[-\frac{1}{2}\left(Z_{P}-Z_{i}\right)\right]\left[\left(d_{i P}^{\prime C}\right)^{-3 / 2}\right]\left[2\left(X_{P}-X_{i}\right)\right]
$$

Simplificando a equação (5.37), tem-se,

$$
\frac{\partial F}{\partial X_{P}}=\left[\frac{-1}{\sqrt{1-\frac{\left(Z_{P}-Z_{i}\right)^{2}}{d_{i P}^{\prime C}}}}\right]\left[\left(X_{P}-X_{i}\right)\left(Z_{P}-Z_{i}\right)\right]\left[\frac{1}{\sqrt{\left(d_{i P}^{\prime C}\right)^{3}}}\right]
$$


A derivada parcial em relação à coordenada $Y_{P}$,

$$
\frac{\partial F}{\partial Y_{P}}=\left[\frac{-1}{\sqrt{1-\frac{\left(Z_{P}-Z_{i}\right)^{2}}{d_{i P}^{\prime}}}}\right]\left[\left(Y_{P}-Y_{i}\right)\left(Z_{P}-Z_{i}\right)\right]\left[\frac{1}{\sqrt{\left(d_{i P}^{\prime C}\right)^{3}}}\right]
$$

Por fim, a derivada parcial em relação à coordenada $Z_{P}$,

$$
\frac{\partial F}{\partial Z_{P}}=\left[\frac{+1}{\sqrt{1-\frac{\left(Z_{P}-Z_{i}\right)^{2}}{d_{i P}^{\prime C}}}}\right]\left[\frac{1}{d_{i P}^{\prime C}}-\left(Z_{P}-Z_{i}\right)^{2}\left(\frac{1}{\sqrt{\left(d_{i P}^{\prime C}\right)^{3}}}\right)\right]
$$

Se as equações (5.34 a 5.40) forem substituídas pela equação (5.30) e os resultados substituídos pela equação (5.29), obtém-se a seguinte equação de observação linearizada, para ângulo vertical de altura:

$$
\begin{aligned}
& L_{\beta_{i P}}+v_{\beta_{i P}^{O}}=\left[\frac{1}{\sqrt{1-\frac{\left(Z_{P}-Z_{i}\right)^{2}}{d_{i P}^{\prime C}}}}\right]\left[\left(X_{P}-X_{i}\right)\left(Z_{P}-Z_{i}\right)\right]\left[\frac{1}{\sqrt{\left(d_{i P}^{\prime C}\right)^{3}}}\right] \delta X_{i}+ \\
& +\frac{1}{\sqrt{1-\frac{\left(Z_{P}-Z_{i}\right)^{2}}{d_{i P}^{\prime C}}}}\left[\left(Y_{P}-Y_{i}\right)\left(Z_{P}-Z_{i}\right)\right]\left[\frac{1}{\sqrt{\left(d_{i P}^{\prime C}\right)^{3}}}\right] \delta Y_{i}+ \\
& +\left[\frac{-1}{\sqrt{1-\frac{\left(Z_{P}-Z_{i}\right)^{2}}{d_{i P}^{\prime C}}}}\right]\left[\left(Z_{P}-Z_{i}\right)^{2}\left(\frac{1}{\sqrt{\left(d_{i P}^{\prime C}\right)^{3}}}-\frac{1}{d_{i P}^{\prime C}}\right)\right] \delta Z_{i}+ \\
& +\left[\frac{-1}{\sqrt{1-\frac{\left(Z_{P}-Z_{i}\right)^{2}}{d_{i P}^{\prime C}}}}\right]\left[\left(X_{P}-X_{i}\right)\left(Z_{P}-Z_{i}\right)\right]\left[\frac{1}{\sqrt{\left(d_{i P}^{\prime C}\right)^{3}}}\right] \delta X_{P}+ \\
& +\left[\frac{-1}{\sqrt{1-\frac{\left(Z_{P}-Z_{i}\right)^{2}}{d_{i P}^{\prime C}}}}\right]\left[\left(Y_{P}-Y_{i}\right)\left(Z_{P}-Z_{i}\right)\right]\left[\frac{1}{\sqrt{\left(d_{i P}^{\prime C}\right)^{3}}}\right] \delta Y_{P}+ \\
& +\left[\frac{1}{\sqrt{1-\frac{\left(Z_{P}-Z_{i}\right)^{2}}{d_{i P}^{\prime C}}}}\right]\left[\frac{1}{d_{i P}^{\prime C}}-\left(Z_{P}-Z_{i}\right)^{2}\left(\frac{1}{\sqrt{\left(d_{i P}^{\prime C}\right)^{3}}}\right)\right] \delta Z_{P}
\end{aligned}
$$


Onde $F\left(P^{0}\right)$ é calculado nos valores aproximados dos parâmetros, $L_{\beta_{i P}}=\beta_{i P}^{O}-\beta_{i P}^{C}$, e

$$
\begin{gathered}
L_{\beta_{i P}}=\beta_{i P}^{O}-\left[\operatorname{sen}^{-1} \frac{\left(\Delta Z_{\left.i P^{0}\right)}\right.}{\left(d_{i P^{0}}^{\prime C}\right)}\right] \\
\beta_{i P}^{C}=F\left(X_{i}^{0}, Y_{i}^{0}, Z_{i}^{0}, X_{P}^{0}, Y_{P}^{0}, Z_{P}^{0}\right)=F\left(P^{0}\right)+L_{\beta_{i P}}\left(P^{0}\right)
\end{gathered}
$$

Desta forma é possível compor a Matriz Jacobiana $A$, para as observações de ângulos verticais de altura, cuja estrutura é representada pela Tabela 5.3.

\begin{tabular}{|c|c|c|c|c|c|c|c|c|c|c|c|c|c|c|c|}
\hline \multirow{2}{*}{$\begin{array}{c}\text { Ângulo vertical de altura } \\
\text { Observações (iP) }\end{array}$} & \multicolumn{15}{|c|}{ Incógnitas } \\
\hline & $\delta X_{2}$ & $\delta Y_{2}$ & $\delta Z_{2}$ & $\delta X_{3}$ & $\delta Y_{3}$ & $\delta Z_{3}$ & $\delta X_{4}$ & $\delta Y_{4}$ & $\delta Z_{4}$ & $\delta X_{5}$ & $\delta Y_{5}$ & $\delta Z_{5}$ & $\delta X_{6}$ & $\delta Y_{6}$ & $\delta Z$ \\
\hline $1-2$ & $P$ & $P$ & $P$ & & & & & & & & & & & & \\
\hline $1-3$ & & & & $P$ & $P$ & $P$ & & & & & & & & & \\
\hline $1-4$ & & & & & & & $P$ & $P$ & $P$ & & & & & & \\
\hline $1-5$ & & & & & & & & & & $P$ & $P$ & $P$ & & & \\
\hline $1-6$ & & & & & & & & & & & & & $P$ & $P$ & $P$ \\
\hline $2-3$ & $i$ & $i$ & $i$ & $P$ & $P$ & $P$ & & & & & & & & & \\
\hline $2-4$ & $i$ & $i$ & $i$ & & & & $P$ & $P$ & $P$ & & & & & & \\
\hline $2-5$ & $i$ & $i$ & $i$ & & & & & & & $P$ & $P$ & $P$ & & & \\
\hline $2-6$ & $i$ & $i$ & $i$ & & & & & & & & & & $P$ & $P$ & $P$ \\
\hline $2-1$ & $i$ & $i$ & $i$ & & & & & & & & & & & & \\
\hline $3-4$ & & & & $i$ & $i$ & $i$ & $P$ & $P$ & $P$ & & & & & & \\
\hline $3-5$ & & & & $i$ & $i$ & $i$ & & & & $P$ & $P$ & $P$ & & & \\
\hline $3-6$ & & & & $i$ & $i$ & $i$ & & & & & & & $P$ & $P$ & $P$ \\
\hline $3-1$ & & & & $i$ & $i$ & $i$ & & & & & & & & & \\
\hline $3-2$ & $P$ & $P$ & $P$ & $i$ & $i$ & $i$ & & & & & & & & & \\
\hline $4-5$ & & & & & & & $i$ & $i$ & $i$ & $P$ & $P$ & $P$ & & & \\
\hline $4-6$ & & & & & & & $i$ & $i$ & $i$ & & & & $P$ & $P$ & $P$ \\
\hline $4-1$ & & & & & & & $i$ & $i$ & $i$ & & & & & & \\
\hline $4-2$ & $P$ & $P$ & $P$ & & & & $i$ & $i$ & $i$ & & & & & & \\
\hline $4-3$ & & & & $P$ & $P$ & $P$ & $i$ & $i$ & $i$ & & & & & & \\
\hline $5-6$ & & & & & & & & & & $i$ & $i$ & $i$ & $P$ & $P$ & $P$ \\
\hline $5-1$ & & & & & & & & & & $i$ & $i$ & $i$ & & & \\
\hline $5-2$ & $P$ & $P$ & $P$ & & & & & & & $i$ & $i$ & $i$ & & & \\
\hline 5-3 & & & & $P$ & $P$ & $P$ & & & & $i$ & $i$ & $i$ & & & \\
\hline 5-4 & & & & & & & $P$ & $P$ & $P$ & $i$ & $i$ & $i$ & & & \\
\hline $6-1$ & & & & & & & & & & & & & $i$ & $i$ & $i$ \\
\hline $6-2$ & $P$ & $P$ & $P$ & & & & & & & & & & $i$ & $i$ & $i$ \\
\hline $6-3$ & & & & $P$ & $P$ & $P$ & & & & & & & $i$ & $i$ & $i$ \\
\hline $6-4$ & & & & & & & $P$ & $P$ & $P$ & & & & $i$ & $i$ & $i$ \\
\hline $6-5$ & & & & & & & & & & $P$ & $P$ & $P$ & $i$ & $i$ & $i$ \\
\hline
\end{tabular}

Tabela 5.3: Estrutura da Matriz A para ângulo vertical de altura.

\subsection{Cálculo de azimute em função de coordenadas espaciais}

A modelagem matemática para cálculo de Azimute, em seu problema inverso, é realizada em função das coordenadas $\left(X_{i}, Y_{i}, X_{P}, X_{P}\right)$, mas para o cálculo espacial de coordenadas, foi estabelecido um modelo para determinação de Azimute na forma inversa, agora em função das coordenadas $\left(X_{i}, Y_{i}, Z_{i}, X_{P}, Y_{P}, Z_{P}\right)$, conforme ilustrado na Figura 5.4. 
Todas as equações de observação estão em função das três coordenadas $\left(X_{P}, Y_{P}, Z_{P}\right)$, o que torna a rede topográfica, de fato, espacial.

Como as equações de observação, para distância inclinada e ângulos verticais zenitais e de altura, são realizadas em função das três coordenadas, foi estabelecida uma equação de observação para azimute, que também leva em consideração as três coordenadas.

Esse azimute será utilizado para as equações de erro no problema inverso, logo se faz necessário considerar a seguinte definição, por meio da projeção $\operatorname{Proj}_{i P}$, como pode ser visto na Figura 5.4.

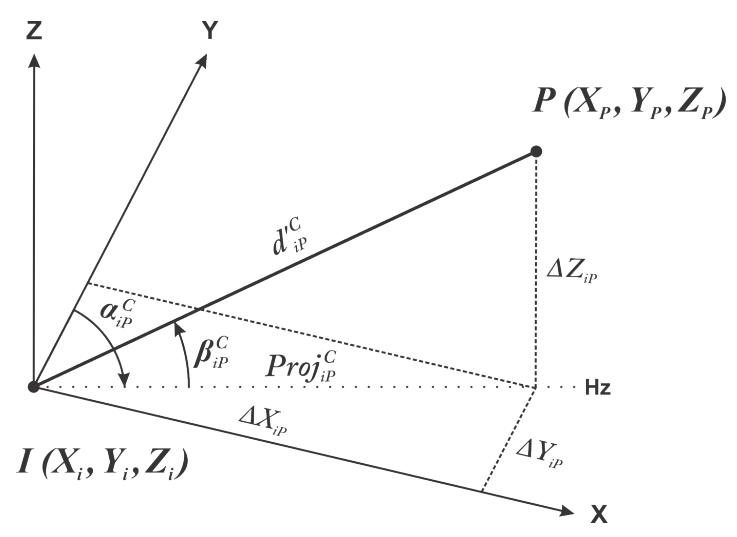

Figura 5.4: Relação trigonométrica de uma observação espacial.

Logo, segue da Figura 5.4 a seguinte definição para o Azimute,

$$
\alpha_{i P}^{C}=g\left(X_{i P}, Y_{i P}, Z_{i P}\right)=\left\{\begin{array}{r}
\operatorname{arctg}\left(\frac{\Delta X_{i P}}{\Delta Y_{i P}}\right) \\
\text { se } Z=0 \\
\operatorname{sen}^{-1}\left(\frac{\Delta X_{i P}}{\sqrt{\left(d_{i P}^{\prime C}\right)^{2}-\left(\Delta Z_{i P}\right)^{2}}}\right) \\
\text { se } Z \neq 0
\end{array}\right.
$$

Pela relação trigonométrica, tem-se:

$$
\begin{gathered}
\operatorname{sen}\left(\alpha_{i P}^{C}\right)=\frac{\Delta X_{i P}}{\operatorname{Proj}_{i P}^{C}} \\
\operatorname{Proj}_{i P}^{C}=\frac{\Delta X_{i P}}{\operatorname{sen}\left(\alpha_{i P}^{C}\right)} \\
\left(d_{i P}^{\prime C}\right)^{2}=\left(\Delta Z_{i P}\right)^{2}+\left(\operatorname{Proj}_{i P}^{C}\right)^{2} \\
\left(\operatorname{Proj}_{i P}^{C}\right)^{2}=\left(d_{i P}^{\prime C}\right)^{2}-\left(\Delta Z_{i P}\right)^{2} \\
\left(\operatorname{Proj}_{i P}^{C}\right)=\sqrt{\left(d_{i P}^{\prime C}\right)^{2}-\left(\Delta Z_{i P}\right)^{2}}
\end{gathered}
$$




$$
\begin{aligned}
& \frac{\Delta X_{i P}}{\operatorname{sen}\left(\alpha_{i P}^{C}\right)}=\sqrt{\left(d_{i P}^{\prime C}\right)^{2}-\left(\Delta Z_{i P}\right)^{2}} \\
& \frac{\Delta X_{i P}}{\sqrt{\left(d_{i P}^{\prime C}\right)^{2}-\left(\Delta Z_{i P}\right)^{2}}}=\operatorname{sen}\left(\alpha_{i P}^{C}\right)
\end{aligned}
$$

Portanto:

$$
\alpha_{i P}^{C}\left(X_{i P}, Y_{i P}, Z_{i P}\right)=\operatorname{sen}^{-1}\left(\frac{\Delta X_{i P}}{\sqrt{\left(d_{i P}^{\prime C}\right)^{2}-\left(\Delta Z_{i P}\right)^{2}}}\right)
$$

\subsection{Equação de observação para ângulo horizontal azimutal}

A rede topográfica espacial que foi aplicada nesta tese, considerou a equação de observação para ângulo horizontal azimutal para a composição da Matriz $A$, mas também foi desenvolvido a equação de observação para ângulo horizontal interno, como pode ser visto na seção 5.6.

Dessa forma, a equação do ângulo horizontal azimutal espacial, em seu problema inverso, na forma paramétrica é dada por:

$$
A z_{i P}^{C}=\alpha_{i P}^{C}+C
$$

Onde,

$$
\alpha_{i P}^{C}=\operatorname{sen}^{-1} \frac{\left(\Delta X_{i P}\right)}{\sqrt{\left(d_{i P}^{\prime C}\right)^{2}-\left(\Delta Z_{i P}\right)^{2}}}
$$

Sendo que $\left(X_{i}, Y_{i}, Z_{i}\right)$ são coordenadas da estação ocupada $I,\left(X_{P}, Y_{P}, Z_{P}\right)$ são as coordenadas da estação visada $P$ e $C$ é a constante que depende em qual quadrante o ponto espacial $P$ se encontra. Como pode ser visto, a Tabela 5.4 relaciona os sinais algébricos do ângulo calculado $\alpha_{i P}^{C}$, na equação (5.52).

Tabela 5.4: Relação entre quadrante, $C$, azimute e o ângulo $\alpha$.

\begin{tabular}{cccccc}
\hline Quadrante & $\Delta X_{i p}$ & $\Delta Y_{i p}$ & $\alpha$ & $C$ & Azimute \\
\hline $\boldsymbol{I}$ & + & + & + & $0^{\circ}$ & $\alpha$ \\
\hline $\boldsymbol{I I}$ & + & - & - & $180^{\circ}$ & $\alpha+180^{\circ}$ \\
\hline $\boldsymbol{I I I}$ & - & - & + & $180^{\circ}$ & $\alpha+180^{\circ}$ \\
\hline $\boldsymbol{I} \boldsymbol{}$ & - & + & - & $360^{\circ}$ & $\alpha+360^{\circ}$ \\
\hline
\end{tabular}


Nota-se que, independentemente do ponto $P$ estar no espaço ou no plano, a análise de quadrante deve ser realizada, utilizando a projeção da mesma, ou seja, a constante $C$ deve considerar o quadrante do plano $(X, Y)$, como ilustra a Figura 5.5.
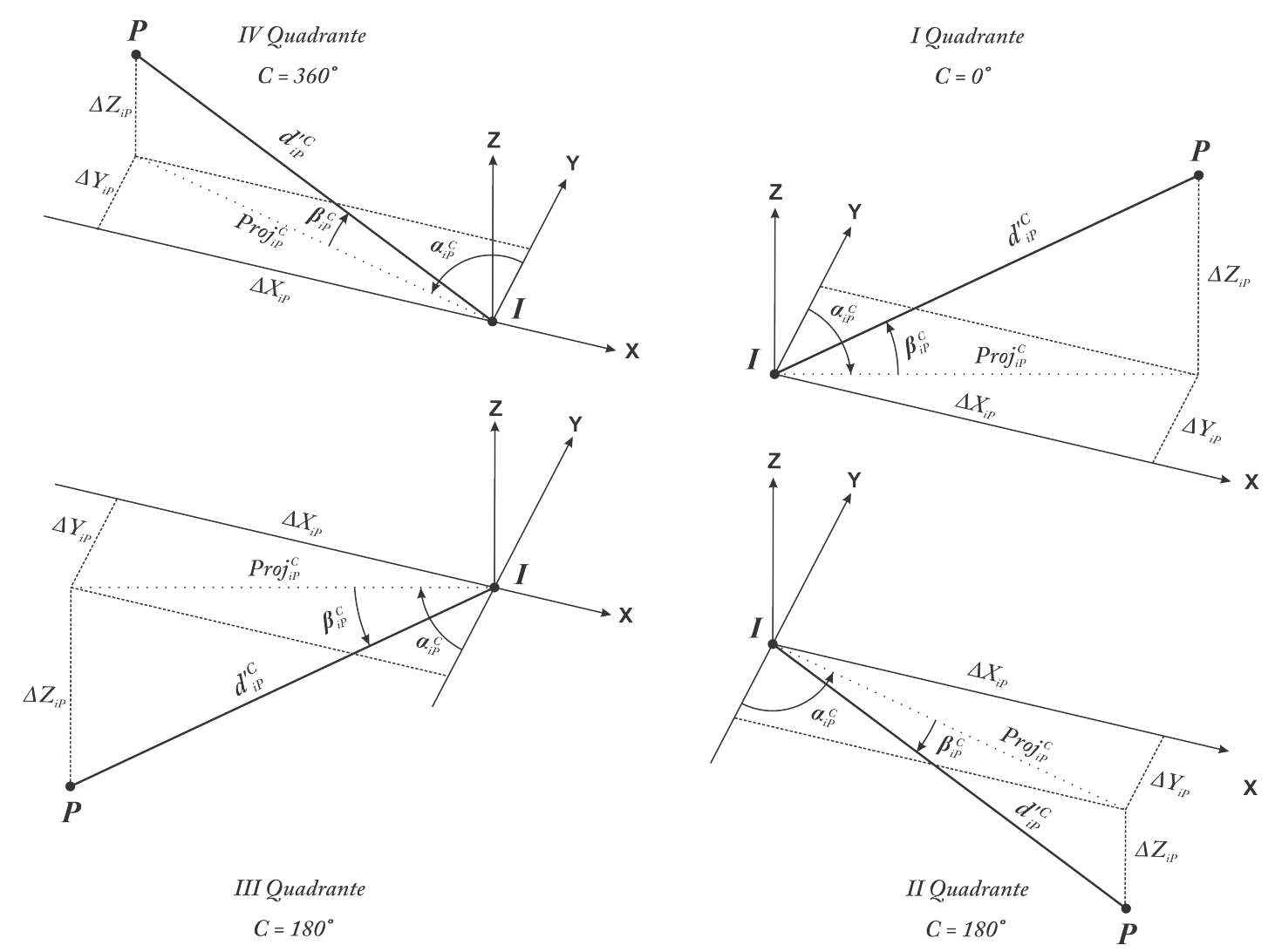

Figura 5.5: Relação entre azimute $A z_{i P}^{C}$ e ângulo $\alpha_{i P}^{C}$ a serem calculados.

Para ajustar as observações de ângulos horizontais azimutais ou triangulações espaciais, utilizando o método paramétrico dos mínimos quadrados, devem ser elaboradas as equações de observação que relacionam as quantidades observadas e seus erros aleatórios, inerentes aos valores mais prováveis para as coordenadas $\left(X_{P}, Y_{P}, Z_{P}\right)$, que são os parâmetros das estações envolvidas. Como pode ser visto na Figura 5.6, a equação (5.53) de ângulo horizontal azimutal pode ser escrita para qualquer observação $A z_{i P}^{O}$, definida por:

$$
\begin{gathered}
A z_{i P}^{O}+v_{A z_{i P}^{O}}=A z_{i P}^{C} \\
A z_{i P}^{O}+v_{A z_{i P}^{O}}=\operatorname{sen}^{-1} \frac{\left(\Delta X_{i P}\right)}{\sqrt{\left(d_{i P}^{\prime C}\right)^{2}-\left(\Delta Z_{i P}\right)^{2}}}+C
\end{gathered}
$$




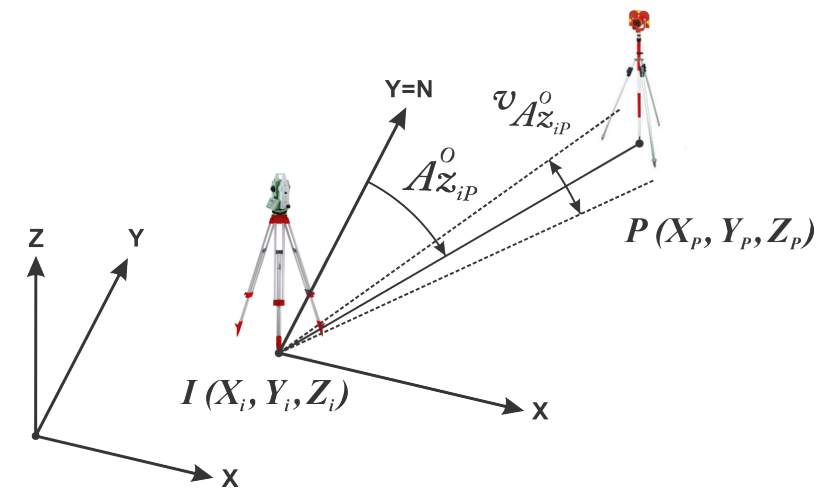

Figura 5.6: Observação de um ângulo horizontal azimutal.

Desta maneira, a fórmula (5.53) do ângulo horizontal azimutal, em função dos parâmetros $\left(X_{i}, Y_{i}, Z_{i}, X_{P}, Y_{P}, Z_{P}\right)$, pode ser reescrita:

$$
F\left(X_{i}, Y_{i}, Z_{i}, X_{P}, Y_{P}, Z_{P}\right)=A z_{i P}^{O}+v_{A z_{i P}^{O}}
$$

Um sistema de equações não lineares, como a equação (5.54), pode ser linearizada e resolvida, utilizando uma série de Taylor de primeira ordem de aproximação. Sendo,

$$
F\left(X_{i}^{0}, Y_{i}^{0}, Z_{i}^{0}, X_{P}^{0}, Y_{P}^{0}, Z_{P}^{0}\right)=F\left(P^{0}\right)
$$

A forma linearizada da equação (5.54) é dada por:

$$
\begin{gathered}
F\left(X_{i}, Y_{i}, Z_{i}, X_{P}, Y_{P}, Z_{P}\right)=F\left(X_{i}^{0}, Y_{i}^{0}, Z_{i}^{0}, X_{P}^{0}, Y_{P}^{0}, Z_{P}^{0}\right)+ \\
+\frac{\partial F\left(P^{0}\right)}{\partial X_{i}} \delta X_{i}+\frac{\partial F\left(P^{0}\right)}{\partial Y_{i}} \delta Y_{i}+0 \delta Z_{i}+ \\
+\frac{\partial F\left(P^{0}\right)}{\partial X_{P}} \delta X_{P}+\frac{\partial F\left(P^{0}\right)}{\partial Y_{P}} \delta Y_{P}+0 \delta Z_{P}
\end{gathered}
$$

Pois,

$$
\frac{\partial F\left(P^{0}\right)}{\partial Z_{i}} \delta Z_{i}=0 \quad \text { e } \quad \frac{\partial F\left(P^{0}\right)}{\partial Z_{P}} \delta Z_{P}=0
$$

Onde $\frac{\partial F\left(P^{0}\right)}{\partial X_{i}} \delta X_{i} ; \frac{\partial F\left(P^{0}\right)}{\partial Y_{i}} \delta Y_{i} ; \frac{\partial F\left(P^{0}\right)}{\partial Z_{i}} \delta Z_{i} ; \frac{\partial F\left(P^{0}\right)}{\partial X_{P}} \delta X_{P} ; \frac{\partial F\left(P^{0}\right)}{\partial Y_{P}} \delta Y_{P} ; \frac{\partial F\left(P^{0}\right)}{\partial Z_{P}} \delta Z_{P}$ são as derivadas parciais de $F$ em relação a $\left(X_{i}, Y_{i}, Z_{i}\right)$ e $\left(X_{P}, Y_{P}, Z_{P}\right)$, respectivamente, avaliadas com os valores de coordenadas espaciais aproximados $\left(X_{i}^{0}, Y_{i}^{0}, Z_{i}^{0}\right)$ e $\left(X_{P}^{0}, Y_{P}^{0}, Z_{P}^{0}\right)$. As coordenadas es- 
paciais $\left(X_{i}, Y_{i}, Z_{i}\right)$ e $\left(X_{P}, Y_{P}, Z_{P}\right)$ são parâmetros desconhecidos, $\left(\delta X_{i}, \delta Y_{i}, \delta Z_{i}\right)$ e $\left(\delta X_{P}, \delta Y_{P}, \delta Z_{P}\right)$ são as correções para os valores de coordenadas de aproximação, tais que:

$$
\begin{array}{rrr}
X_{i}=X_{i}^{0}+\delta X_{i} & Y_{i}=Y_{i}^{0}+\delta Y_{i} & Z_{i}=Z_{i}^{0}+\delta Z_{i} \\
X_{P}=X_{P}^{0}+\delta X_{P} & Y_{P}=Y_{P}^{0}+\delta Y_{P} & Z_{P}=Z_{P}^{0}+\delta Z_{P}
\end{array}
$$

Para determinar as derivadas parciais da equação (5.55), é necessário conhecer a derivada da função inversa de $\operatorname{sen}^{-1}$ em relação à $x$, a qual se sabe que é:

$$
\frac{d}{d x} \operatorname{sen}^{-1} u=\frac{1}{\sqrt{1-u^{2}}} \frac{d u}{d x}
$$

Utilizando a equação (5.57), o procedimento para determinar a derivada parcial $\partial F / \partial X_{i}$ é demonstrado como segue:

$$
\begin{gathered}
\frac{\partial F}{\partial X_{i}}=\frac{1}{\sqrt{1-\frac{\left(X_{P}-X_{i}\right)^{2}}{\left(d_{i P}^{\prime \prime}\right)^{2}-\left(Z_{P}-Z_{i}\right)^{2}}}} \cdot \\
\frac{(-1)\left[\sqrt{\left(d_{i P}^{\prime C}\right)^{2}-\left(Z_{P}-Z_{i}\right)^{2}}-\left(X_{P}-X_{i}\right)^{2}\right] \frac{1}{2}\left[\left(d_{i P}^{\prime C}\right)^{2}-\left(Z_{P}-Z_{i}\right)^{2}\right]^{-1 / 2}-\left[2\left(X_{P}-X_{i}\right)\right]}{\left[\left(d_{i P}^{\prime C}\right)^{2}-\left(Z_{P}-Z_{i}\right)^{2}\right]}
\end{gathered}
$$

Simplificando a equação (5.58), tem-se:

$$
\begin{gathered}
\frac{\partial F}{\partial X_{i}}=\frac{1}{\sqrt{1-\frac{\left(X_{P}-X_{i}\right)^{2}}{\left(d_{i P}^{\prime C}\right)^{2}-\left(Z_{P}-Z_{i}\right)^{2}}}} \cdot \\
\frac{\left[\left(X_{P}-X_{i}\right)^{2}\right]\left[\left(d_{i P}^{\prime C}\right)^{2}-\left(Z_{P}-Z_{i}\right)^{2}\right]^{-1 / 2}-\left[\left(d_{i P}^{\prime C}\right)^{2}-\left(Z_{P}-Z_{i}\right)^{2}\right]^{1 / 2}}{\left[\left(d_{i P}^{\prime C}\right)^{2}-\left(Z_{P}-Z_{i}\right)^{2}\right]}
\end{gathered}
$$

Com o emprego do mesmo procedimento, ou de forma análoga, obtém-se as derivadas parciais remanescentes:

$$
\begin{gathered}
\frac{\partial F}{\partial Y_{i}}=\frac{1}{\sqrt{1-\frac{\left(X_{P}-X_{i}\right)^{2}}{\left(d_{i P}^{\prime C}\right)^{2}-\left(Z_{P}-Z_{i}\right)^{2}}}} \cdot \frac{\left[\left(X_{P}-X_{i}\right)\left(X_{P}-X_{i}\right)\right]}{\left[\left(d_{i P}^{\prime C}\right)^{2}-\left(Z_{P}-Z_{i}\right)\right]^{3 / 2}} \\
\frac{\partial F}{\partial Z_{i}}=0 \\
\frac{\partial F}{\partial X_{P}}=\frac{1}{\sqrt{1-\frac{\left(X_{P}-X_{i}\right)^{2}}{\left(d_{i P}^{\prime}\right)^{2}-\left(Z_{P}-Z_{i}\right)^{2}}}} .
\end{gathered}
$$




$$
\begin{gathered}
\frac{\left[\left(d_{i P}^{\prime C}\right)^{2}-\left(Z_{P}-Z_{i}\right)^{2}\right]^{1 / 2}-\left[\left(X_{P}-X_{i}\right)\left(Y_{P}-Y_{i}\right)\right]\left[\left(d_{i P}^{\prime C}\right)^{2}-\left(Z_{P}-Z_{i}\right)^{2}\right]^{-1 / 2}}{\left[\left(d_{i P}^{\prime C}\right)^{2}-\left(Z_{P}-Z_{i}\right)^{2}\right]} \\
\frac{\partial F}{\partial Y_{P}}=\frac{-\left(X_{P}-X_{i}\right)\left(Y_{P}-Y_{i}\right)}{\sqrt{1-\frac{\left(X_{P}-X_{i}\right)^{2}}{\left(d_{i P}^{\prime C}\right)^{2}-\left(Z_{P}-Z_{i}\right)^{2}}}} \cdot\left[\left(d_{i P}^{\prime C}\right)^{2}-\left(Z_{P}-Z_{i}\right)\right]^{-3 / 2} \\
\frac{\partial F}{\partial Z_{P}}=0
\end{gathered}
$$

Se as equações (5.59 a 5.65) forem substituídas pela equação (5.55) e os resultados substituídos pela equação (5.54), obtém-se a seguinte equação de observação linearizada, para ângulo horizontal azimutal:

$$
\begin{aligned}
& L_{A z_{i P}}+v_{A z_{i P}^{O}}=\frac{1}{\sqrt{1-\frac{\left(X_{P}-X_{i}\right)^{2}}{\left(d_{i P}^{\prime C}\right)^{2}-\left(Z_{P}-Z_{i}\right)^{2}}}} . \\
& \cdot \frac{\left[\left(X_{P}-X_{i}\right)^{2}\right]\left[\left(d_{i P}^{\prime C}\right)^{2}-\left(Z_{P}-Z_{i}\right)^{2}\right]^{-1 / 2}-\left[\left(d_{i P}^{\prime C}\right)^{2}-\left(Z_{P}-Z_{i}\right)^{2}\right]^{1 / 2}}{\left[\left(d_{i P}^{\prime C}\right)^{2}-\left(Z_{P}-Z_{i}\right)^{2}\right]} \delta X_{i}+ \\
& +\frac{1}{\sqrt{1-\frac{\left(X_{P}-X_{i}\right)^{2}}{\left(d_{i P}^{\prime C}\right)^{2}-\left(Z_{P}-Z_{i}\right)^{2}}}} \cdot \frac{\left[\left(X_{P}-X_{i}\right)\left(X_{P}-X_{i}\right)\right]}{\left[\left(d_{i P}^{\prime C}\right)^{2}-\left(Z_{P}-Z_{i}\right)\right]^{3 / 2}} \delta Y_{i}+ \\
& +0 \delta Z_{i}+ \\
& +\frac{1}{\sqrt{1-\frac{\left(X_{P}-X_{i}\right)^{2}}{\left(d_{i P}^{\prime}\right)^{2}-\left(Z_{P}-Z_{i}\right)^{2}}}} \\
& \cdot \frac{\left[\left(d_{i P}^{\prime C}\right)^{2}-\left(Z_{P}-Z_{i}\right)^{2}\right]^{1 / 2}-\left[\left(X_{P}-X_{i}\right)\left(Y_{P}-Y_{i}\right)\right]\left[\left(d_{i P}^{\prime C}\right)^{2}-\left(Z_{P}-Z_{i}\right)^{2}\right]^{-1 / 2}}{\left[\left(d_{i P}^{\prime C}\right)^{2}-\left(Z_{P}-Z_{i}\right)^{2}\right]} \delta X_{P}+ \\
& +\frac{-\left(X_{P}-X_{i}\right)\left(Y_{P}-Y_{i}\right)}{\sqrt{1-\frac{\left(X_{P}-X_{i}\right)^{2}}{\left(d_{i P}^{\prime C}\right)^{2}-\left(Z_{P}-Z_{i}\right)^{2}}}} \cdot\left[\left(d_{i P}^{\prime C}\right)^{2}-\left(Z_{P}-Z_{i}\right)\right]^{-3 / 2} \delta Y_{P}+ \\
& +0 \delta Z_{P}
\end{aligned}
$$

Onde $F\left(P_{0}\right)$ é calculado nos valores aproximados dos parâmetros, $L_{A z_{i P}}=A z_{i P}^{O}-A z_{i P}^{C}$, e

$$
\begin{gathered}
L_{A z_{i P}}=A z_{i P}^{O}-\left[\operatorname{sen}^{-1} \frac{\left(\Delta X_{i P^{0}}\right)}{\sqrt{\left(d_{i P^{0}}^{\prime C}\right)^{2}-\left(\Delta Z_{i P^{0}}\right)^{2}}}+C\right] \\
A z_{i P}^{C}=F\left(X_{i}^{0}, Y_{i}^{0}, Z_{i}^{0}, X_{P}^{0}, Y_{P}^{0}, Z_{P}^{0}\right)=F\left(P^{0}\right)+L_{A z_{i P}}\left(P^{0}\right)
\end{gathered}
$$


Desta forma, é possível compor a Matriz Jacobiana $A$, para as observações de ângulos horizontais azimutais, cuja estrutura é representada pela Tabela 5.5.

Tabela 5.5: Estrutura da Matriz A para ângulo horizontal azimutal.

\begin{tabular}{|c|c|c|c|c|c|c|c|c|c|c|c|c|c|c|c|}
\hline \multirow{2}{*}{$\begin{array}{c}\text { Ângulo horizontal azimutal } \\
\text { Observações (iP) }\end{array}$} & \multicolumn{15}{|c|}{ Incógnitas } \\
\hline & $\delta X_{2}$ & $\delta Y_{2}$ & $\delta Z_{2}$ & $\delta X_{3}$ & $\delta Y_{3}$ & $\delta Z_{3}$ & $\delta X_{4}$ & $\delta Y_{4}$ & $\delta Z_{4}$ & $\delta X_{5}$ & $\delta Y_{5}$ & $\delta Z_{5}$ & $\delta X_{6}$ & $\delta Y_{6}$ & $\delta Z_{6}$ \\
\hline $1-2$ & $P$ & $P$ & $P$ & & & & & & & & & & & & \\
\hline $1-3$ & & & & $P$ & $P$ & $P$ & & & & & & & & & \\
\hline $1-4$ & & & & & & & $P$ & $P$ & $P$ & & & & & & \\
\hline $1-5$ & & & & & & & & & & $P$ & $P$ & $P$ & & & \\
\hline $1-6$ & & & & & & & & & & & & & $P$ & $P$ & $P$ \\
\hline $2-3$ & $i$ & $i$ & $i$ & $P$ & $P$ & $P$ & & & & & & & & & \\
\hline $2-4$ & $i$ & $i$ & $i$ & & & & $P$ & $P$ & $P$ & & & & & & \\
\hline $2-5$ & $i$ & $i$ & $i$ & & & & & & & $P$ & $P$ & $P$ & & & \\
\hline $2-6$ & $i$ & $i$ & $i$ & & & & & & & & & & $P$ & $P$ & $P$ \\
\hline $2-1$ & $i$ & $i$ & $i$ & & & & & & & & & & & & \\
\hline $3-4$ & & & & $i$ & $i$ & $i$ & $P$ & $P$ & $P$ & & & & & & \\
\hline $3-5$ & & & & $i$ & $i$ & $i$ & & & & $P$ & $P$ & $P$ & & & \\
\hline $3-6$ & & & & $i$ & $i$ & $i$ & & & & & & & $P$ & $P$ & $P$ \\
\hline $3-1$ & & & & $i$ & $i$ & $i$ & & & & & & & & & \\
\hline $3-2$ & $P$ & $P$ & $P$ & $i$ & $i$ & $i$ & & & & & & & & & \\
\hline $4-5$ & & & & & & & $i$ & $i$ & $i$ & $P$ & $P$ & $P$ & & & \\
\hline $4-6$ & & & & & & & $i$ & $i$ & $i$ & & & & $P$ & $P$ & $P$ \\
\hline $4-1$ & & & & & & & $i$ & $i$ & $i$ & & & & & & \\
\hline $4-2$ & $P$ & $P$ & $P$ & & & & $i$ & $i$ & $i$ & & & & & & \\
\hline $4-3$ & & & & $P$ & $P$ & $P$ & $i$ & $i$ & $i$ & & & & & & \\
\hline $5-6$ & & & & & & & & & & $i$ & $i$ & $i$ & $P$ & $P$ & $P$ \\
\hline $5-1$ & & & & & & & & & & $i$ & $i$ & $i$ & & & \\
\hline $5-2$ & $P$ & $P$ & $P$ & & & & & & & $i$ & $i$ & $i$ & & & \\
\hline $5-3$ & & & & $P$ & $P$ & $P$ & & & & $i$ & $i$ & $i$ & & & \\
\hline $5-4$ & & & & & & & $P$ & $P$ & $P$ & $i$ & $i$ & $i$ & & & \\
\hline $6-1$ & & & & & & & & & & & & & $i$ & $i$ & $i$ \\
\hline $6-2$ & $P$ & $P$ & $P$ & & & & & & & & & & $i$ & $i$ & $i$ \\
\hline $6-3$ & & & & $P$ & $P$ & $P$ & & & & & & & $i$ & $i$ & $i$ \\
\hline $6-4$ & & & & & & & $P$ & $P$ & $P$ & & & & $i$ & $i$ & $i$ \\
\hline $6-5$ & & & & & & & & & & $P$ & $P$ & $P$ & $i$ & $i$ & $i$ \\
\hline
\end{tabular}

\subsection{Equação de observação para ângulo horizontal interno}

Foi desenvolvido também, outra forma para compor a Matriz $A$ da rede topográfica espacial, a qual pode ser realizada por meio da equação de observação para ângulo horizontal interno, porém, não foi aplicada nesta tese.

A Figura 5.7 ilustra a geometria para uma observação angular horizontal interna, em que $R$ é a estação visada à ré, $V$ a estação visada à vante e $I$ a estação ocupada pelo instrumento e uma equação de observação de ângulo horizontal interno pode ser escrita como a diferença entre duas observações de azimute e, assim, obtêm-se ângulos internos no sentido horário. Já o problema inverso, na forma paramétrica, para a equação do ângulo horizontal interno, que considera os ângulos horizontais azimutais, calculados em função de três coordenadas, tais ângulos horizontais internos podem ser representados pela equação (5.53) de observação azimutal, de acordo com a Figura 5.6. 


$$
\angle R I V=\left(A z_{i v}^{O}+v_{A z_{i v}^{O}}\right)-\left(A z_{i r}^{O}+v_{A z_{i r}^{O}}\right)=A z_{i v}^{C}-A z_{i r}^{C}+D=\theta_{\text {riv }}^{O}+v_{\theta_{\text {riv }}^{O}}
$$

Dessa forma, é possível obter a equação (5.69),

$$
\angle R I V=\operatorname{sen}^{-1} \frac{\left(\Delta X_{i v}\right)}{\sqrt{\left(d_{i v}^{\prime C}\right)^{2}-\left(\Delta Z_{i v}\right)^{2}}}-\operatorname{sen}^{-1} \frac{\left(\Delta X_{i r}\right)}{\sqrt{\left(d_{i r}^{\prime C}\right)^{2}-\left(\Delta Z_{i r}\right)^{2}}}+D=\theta_{\text {riv }}^{O}+v_{\theta_{\text {riv }}^{O}}
$$

Onde $\theta_{\text {riv }}^{O}$ é a observação do ângulo horizontal interno no sentido horário, $v_{\theta_{\text {riv }}}$ é o resíduo nesta observação angular, $\left(X_{r}, Y_{r}, Z_{r}\right)$ é o valor mais provável para as coordenadas espaciais visadas à ré na estação $R,\left(X_{i}, Y_{i}, Z_{i}\right)$ é o valor mais provável para as coordenadas espaciais na estação ocupada pelo instrumento $I,\left(X_{v}, Y_{v}, Z_{v}\right)$ é o valor mais provável para as coordenadas espaciais visadas à vante na estação $V$, e $D$ é a constante que depende do quadrante no qual a visada à ré e sua respectiva visada à vante ocorrem.

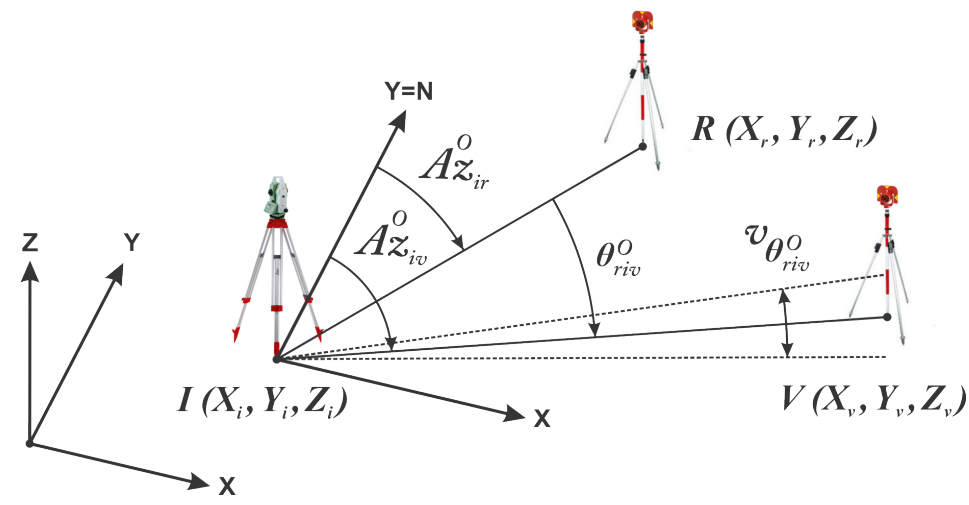

Figura 5.7: Relação entre um ângulo horizontal interno e dois azimutes.

Este termo pode ser computado como a diferença entre o termo $C$ da equação (5.69), aplicada na ré e na vante do azimute, isto é,

$$
D=C_{i v}-C_{i r}
$$

A equação (5.69) é uma função não linear para $\left(X_{r}, Y_{r}, Z_{r}, X_{i}, Y_{i}, Z_{i}, X_{v}, Y_{v}, Z_{v}\right)$ e pode ser reescrita como,

$$
F\left(X_{r}, Y_{r}, Z_{r}, X_{i}, Y_{i}, Z_{i}, X_{v}, Y_{v}, Z_{v}\right)=\theta_{r i v}^{O}+v_{\theta_{r i v}^{O}}
$$

A equação (5.70), expressa como uma expansão linear de série Taylor de primeira ordem, é dada por, 


$$
\begin{aligned}
F\left(X_{r}, Y_{r}, Z_{r}, X_{i}, Y_{i}, Z_{i}, X_{v}, Y_{v}, Z_{v}\right)=F\left(X_{r}^{0}, Y_{r}^{0}, Z_{r}^{0}, X_{i}^{0}, Y_{i}^{0}, Z_{i}^{0}, X_{v}^{0}, Y_{v}^{0}, Z_{v}^{0}\right)+ \\
+\frac{\partial F\left(P^{0}\right)}{\partial X_{r}} \delta X_{r}+\frac{\partial F\left(P_{0}\right)}{\partial Y_{r}} \delta Y_{r}+0 \delta Z_{r}+ \\
+\frac{\partial F\left(P^{0}\right)}{\partial X_{i}} \delta X_{i}+\frac{\partial F\left(P^{0}\right)}{\partial Y_{i}} \delta Y_{i}+0 \delta Z_{i}+ \\
+\frac{\partial F\left(P^{0}\right)}{\partial X_{v}} \delta X_{v}+\frac{\partial F\left(P^{0}\right)}{\partial Y_{v}} \delta Y_{v}+0 \delta Z_{v}
\end{aligned}
$$

Pois,

$$
\frac{\partial F\left(P^{0}\right)}{\partial Z_{r}} \delta Z_{r}=0 \quad \text { e } \quad \frac{\partial F\left(P^{0}\right)}{\partial Z_{i}} \delta Z_{i}=0 \quad \text { e } \quad \frac{\partial F\left(P^{0}\right)}{\partial Z_{v}} \delta Z_{v}=0
$$

Onde $\frac{\partial F\left(P^{0}\right)}{\partial X_{r}} ; \frac{\partial F\left(P^{0}\right)}{\partial Y_{r}} ; \frac{\partial F\left(P^{0}\right)}{\partial Z_{r}} ; \frac{\partial F\left(P^{0}\right)}{\partial X_{i}} ; \frac{\partial F\left(P^{0}\right)}{\partial Y_{i}} ; \frac{\partial F\left(P^{0}\right)}{\partial Z_{i}} ; \frac{\partial F\left(P^{0}\right)}{\partial X_{v}} ; \frac{\partial F\left(P^{0}\right)}{\partial Y_{v}} ; \frac{\partial F\left(P^{0}\right)}{\partial Z_{v}}$ são as derivadas parciais de $F$ em relação a $\left(X_{r}, Y_{r}, Z_{r}\right),\left(X_{i}, Y_{i}, Z_{i}\right)$ e $\left(X_{v}, Y_{v}, Z_{v}\right)$, respectivamente, avaliadas com os seus valores de coordenadas espaciais aproximados $\left(X_{r}^{0}, Y_{r}^{0}, Z_{r}^{0}\right),\left(X_{i}^{0}, Y_{i}^{0}, Z_{i}^{0}\right)$ e $\left(X_{v}^{0}, Y_{v}^{0}, Z_{v}^{0}\right)$. As coordenadas espaciais $\left(X_{r}, Y_{r}, Z_{r}\right),\left(X_{i}, Y_{i}, Z_{i}\right)$ e $\left(X_{v}, Y_{v}, Z_{v}\right)$ são parâmetros desconhecidos, $\left(\delta X_{r}, \delta Y_{r}, \delta Z_{r}\right),\left(\delta X_{i}, \delta Y_{i}, \delta Z_{i}\right)$ e $\left(\delta X_{v}, \delta Y_{v}, \delta Z_{v}\right)$ são as correções para os valores de coordenadas de aproximação, tais que:

$$
\begin{array}{lrl}
X_{r}=X_{r}^{0}+\delta X_{r} & Y_{r}=Y_{r}^{0}+\delta Y_{r} & Z_{r}=Z_{r}^{0}+\delta Z_{r} \\
X_{i}=X_{i}^{0}+\delta X_{i} & Y_{i}=Y_{i}^{0}+\delta Y_{i} & Z_{i}=Z_{i}^{0}+\delta Z_{i} \\
X_{v}=X_{v}^{0}+\delta X_{v} & Y_{v}=Y_{v}^{0}+\delta Y_{v} & Z_{v}=Z_{v}^{0}+\delta Z_{v}
\end{array}
$$

Se as derivadas parciais da função $F$ forem substituídas pela equação (5.71) e os resultados substituídos pela equação (5.70), obtém-se a seguinte equação de observação linearizada, para 
ângulo horizontal interno:

$$
\begin{aligned}
& L_{\theta_{\text {riv }}}+v_{\theta_{\text {riv }}^{O}}=\frac{1}{\sqrt{1-\frac{\left(X_{r}-X_{i}\right)^{2}}{\left(d_{i r}^{\prime C}\right)^{2}-\left(Z_{r}-Z_{i}\right)^{2}}}} . \\
& \frac{\left[\left(X_{r}-X_{i}\right)^{2}\right]\left[\left(d_{i r}^{\prime C}\right)^{2}-\left(Z_{r}-Z_{i}\right)^{2}\right]^{-1 / 2}-\left[\left(d_{i r}^{\prime C}\right)^{2}-\left(Z_{r}-Z_{i}\right)^{2}\right]^{1 / 2}}{\left[\left(d_{i r}^{\prime C}\right)^{2}-\left(Z_{r}-Z_{i}\right)^{2}\right]} \delta X_{i r}+ \\
& +\frac{1}{\sqrt{1-\frac{\left(X_{r}-X_{i}\right)^{2}}{\left(d_{i r}^{\prime C}\right)^{2}-\left(Z_{r}-Z_{i}\right)^{2}}}} \cdot \frac{\left[\left(X_{r}-X_{i}\right)\left(X_{r}-X_{i}\right)\right]}{\left[\left(d_{i r}^{\prime C}\right)^{2}-\left(Z_{r}-Z_{i}\right)\right]^{3 / 2}} \delta Y_{i r}+0 \delta Z_{i r}+ \\
& \frac{1}{\sqrt{1-\frac{\left(X_{r}-X_{i}\right)^{2}}{\left(d_{i r}^{\prime C}\right)^{2}-\left(Z_{r}-Z_{i}\right)^{2}}}} \\
& \cdot \frac{\left[\left(d_{i r}^{\prime C}\right)^{2}-\left(Z_{r}-Z_{i}\right)^{2}\right]^{1 / 2}-\left[\left(X_{r}-X_{i}\right)\left(Y_{r}-Y_{i}\right)\right]\left[\left(d_{i r}^{\prime C}\right)^{2}-\left(Z_{r}-Z_{i}\right)^{2}\right]^{-1 / 2}}{\left[\left(d_{i r}^{\prime C}\right)^{2}-\left(Z_{r}-Z_{i}\right)^{2}\right]} \delta X_{i r}- \\
& -\frac{1}{\sqrt{1-\frac{\left(X_{v}-X_{i}\right)^{2}}{\left(d_{i v}^{\prime C}\right)^{2}-\left(Z_{v}-Z_{i}\right)^{2}}}} \\
& \cdot \frac{\left[\left(d_{i v}^{\prime C}\right)^{2}-\left(Z_{v}-Z_{i}\right)^{2}\right]^{1 / 2}-\left[\left(X_{v}-X_{i}\right)\left(Y_{v}-Y_{i}\right)\right]\left[\left(d_{i v}^{\prime C}\right)^{2}-\left(Z_{v}-Z_{i}\right)^{2}\right]^{-1 / 2}}{\left[\left(d_{i v}^{\prime C}\right)^{2}-\left(Z_{v}-Z_{i}\right)^{2}\right]} \delta X_{i v}+ \\
& +\frac{-\left(X_{r}-X_{i}\right)\left(Y_{r}-Y_{i}\right)}{\sqrt{1-\frac{\left(X_{r}-X_{i}\right)^{2}}{\left(d_{i r}^{C}\right)^{2}-\left(Z_{r}-Z_{i}\right)^{2}}}} \cdot\left[\left(d_{i r}^{\prime C}\right)^{2}-\left(Z_{r}-Z_{i}\right)\right]^{-3 / 2} \delta Y_{i r}- \\
& -\frac{-\left(X_{v}-X_{i}\right)\left(Y_{v}-Y_{i}\right)}{\sqrt{1-\frac{\left(X_{v}-X_{i}\right)^{2}}{\left(d_{i v}^{\prime C}\right)^{2}-\left(Z_{v}-Z_{i}\right)^{2}}}} \cdot\left[\left(d_{i v}^{\prime C}\right)^{2}-\left(Z_{v}-Z_{i}\right)\right]^{-3 / 2} \delta Y_{i v}+0 \delta Z_{i r}-0 \delta Z_{i v}+ \\
& +\frac{1}{\sqrt{1-\frac{\left(X_{v}-X_{i}\right)^{2}}{\left(d_{i v}^{\prime C}\right)^{2}-\left(Z_{v}-Z_{i}\right)^{2}}}} \\
& \frac{\left[\left(d_{i v}^{\prime C}\right)^{2}-\left(Z_{v}-Z_{i}\right)^{2}\right]^{1 / 2}-\left[\left(X_{v}-X_{i}\right)\left(Y_{v}-Y_{i}\right)\right]\left[\left(d_{i v}^{\prime C}\right)^{2}-\left(Z_{v}-Z_{i}\right)^{2}\right]^{-1 / 2}}{\left[\left(d_{i v}^{\prime C}\right)^{2}-\left(Z_{v}-Z_{i}\right)^{2}\right]} \delta X_{i v}+ \\
& +\frac{-\left(X_{v}-X_{i}\right)\left(Y_{v}-Y_{i}\right)}{\sqrt{1-\frac{\left(X_{v}-X_{i}\right)^{2}}{\left(d_{i v}^{\prime \prime}\right)^{2}-\left(Z_{v}-Z_{i}\right)^{2}}}} \cdot\left[\left(d_{i v}^{\prime C}\right)^{2}-\left(Z_{v}-Z_{i}\right)\right]^{-3 / 2} \delta Y_{i v}+0 \delta Z_{i v}
\end{aligned}
$$

Onde,

$$
L_{\theta_{\text {riv }}}=\theta_{\text {riv }}^{O}-\theta_{\text {riv }}^{C}
$$




$$
\theta_{\text {riv0 }}^{C}=\operatorname{sen}^{-1} \frac{\left(\Delta X_{i v^{0}}\right)}{\sqrt{\left(d_{i v^{\prime}}^{C}\right)^{2}-\left(\Delta Z_{i v^{0}}\right)^{2}}}-\operatorname{sen}^{-1} \frac{\left(\Delta X_{i r^{0}}\right)}{\sqrt{\left(d_{i r^{0}}^{\prime C}\right)^{2}-\left(\Delta Z_{i r^{0}}\right)^{2}}}+D
$$

Ao formular a equação de observação do ângulo, é importante lembrar que $I$ é atribuído à estação do instrumento, $R$ à estação de visada a ré e $V$ à estação de visada a vante. Esta designação de estação deve ser seguida estritamente ao empregar a equação (5.71).

Para compor a Matriz Jacobiana $A$ das observações dos ângulos horizontais internos, foi considerado, nesta tese, $r$ como sendo o valor de $\partial F / \partial X_{r}, i$ como sendo o valor de $\partial F / \partial X_{i}$, e $v$ como sendo o valor de $\partial F / \partial X_{v}$ em seus respectivos pontos. Sendo assim, a estrutura da Matriz A é representada pela Tabela 5.6.

Tabela 5.6: Estrutura da Matriz A para ângulo horizontal interno.

\begin{tabular}{|c|c|c|c|c|c|c|c|c|c|c|c|c|c|c|c|}
\hline \multirow{2}{*}{$\begin{array}{c}\text { Ângulo horizontal interno } \\
\text { Observações (riv) }\end{array}$} & \multicolumn{15}{|c|}{ Incógnitas } \\
\hline & $\delta X_{2}$ & $\delta Y_{2}$ & $\delta Z_{2}$ & $\delta X_{3}$ & $\delta Y_{3}$ & $\delta Z_{3}$ & $\delta X_{4}$ & $\delta Y_{4}$ & $\delta Z_{4}$ & $\delta X_{5}$ & $\delta Y_{5}$ & $\delta Z_{5}$ & $\delta X_{6}$ & $\delta Y_{6}$ & $\delta Z_{6}$ \\
\hline $6-1-2$ & $v$ & $v$ & $v$ & & & & & & & & & & $r$ & $r$ & $r$ \\
\hline $6-1-3$ & & & & $v$ & $v$ & $v$ & & & & & & & $r$ & $r$ & $r$ \\
\hline $6-1-4$ & & & & & & & $v$ & $v$ & $v$ & & & & $r$ & $r$ & $r$ \\
\hline $6-1-5$ & & & & & & & & & & $v$ & $v$ & $v$ & $r$ & $r$ & $r$ \\
\hline $6-1-6$ & & & & & & & & & & & & & $r v$ & $r v$ & $r v$ \\
\hline $1-2-3$ & $i$ & $i$ & $i$ & $v$ & $v$ & $v$ & & & & & & & & & \\
\hline $1-2-4$ & $i$ & $i$ & $i$ & & & & $v$ & $v$ & $v$ & & & & & & \\
\hline $1-2-5$ & $i$ & $i$ & $i$ & & & & & & & $v$ & $v$ & $v$ & & & \\
\hline $1-2-6$ & $i$ & $i$ & $i$ & & & & & & & & & & $v$ & $v$ & $v$ \\
\hline $1-2-1$ & $i$ & $i$ & $i$ & & & & & & & & & & & & \\
\hline $2-3-4$ & $r$ & $r$ & $r$ & $i$ & $i$ & $i$ & $v$ & $v$ & $v$ & & & & & & \\
\hline $2-3-5$ & $r$ & $r$ & $r$ & $i$ & $i$ & $i$ & & & & $v$ & $v$ & $v$ & & & \\
\hline $2-3-6$ & $r$ & $r$ & $r$ & $i$ & $i$ & $i$ & & & & & & & $v$ & $v$ & $v$ \\
\hline $2-3-1$ & $r$ & $r$ & $r$ & $i$ & $i$ & $i$ & & & & & & & & & \\
\hline $2-3-2$ & $r v$ & $r v$ & $r v$ & $i$ & $i$ & $i$ & & & & & & & & & \\
\hline $3-4-5$ & & & & $r$ & $r$ & $r$ & $i$ & $i$ & $i$ & $v$ & $v$ & $v$ & & & \\
\hline $3-4-6$ & & & & $r$ & $r$ & $r$ & $i$ & $i$ & $i$ & & & & $v$ & $v$ & $v$ \\
\hline $3-4-1$ & & & & $r$ & $r$ & $r$ & $i$ & $i$ & $i$ & & & & & & \\
\hline $3-4-2$ & $v$ & $v$ & $v$ & $r$ & $r$ & $r$ & $i$ & $i$ & $i$ & & & & & & \\
\hline $3-4-3$ & & & & $r v$ & $r v$ & $r v$ & $i$ & $i$ & $i$ & & & & & & \\
\hline $4-5-6$ & & & & & & & $r$ & $r$ & $r$ & $i$ & $i$ & $i$ & $v$ & $v$ & $v$ \\
\hline $4-5-1$ & & & & & & & $r$ & $r$ & $r$ & $i$ & $i$ & $i$ & & & \\
\hline $4-5-2$ & $v$ & $v$ & $v$ & & & & $r$ & $r$ & $r$ & $i$ & $i$ & $i$ & & & \\
\hline $4-5-6$ & & & & $v$ & $v$ & $v$ & $r$ & $r$ & $r$ & $i$ & $i$ & $i$ & & & \\
\hline $4-5-4$ & & & & & & & $r v$ & $r v$ & $r v$ & $i$ & $i$ & $i$ & & & \\
\hline $5-6-1$ & & & & & & & & & & $r$ & $r$ & $r$ & $i$ & $i$ & $i$ \\
\hline $5-6-2$ & $v$ & $v$ & $v$ & & & & & & & $r$ & $r$ & $r$ & $i$ & $i$ & $i$ \\
\hline $5-6-3$ & & & & $v$ & $v$ & $v$ & & & & $r$ & $r$ & $r$ & $i$ & $i$ & $i$ \\
\hline $5-6-4$ & & & & & & & $v$ & $v$ & $v$ & $r$ & $r$ & $r$ & $i$ & $i$ & $i$ \\
\hline $5-6-5$ & & & & & & & & & & $r v$ & $r v$ & $r v$ & $i$ & $i$ & $i$ \\
\hline
\end{tabular}

Portanto, após o desenvolvimento das equações de observações para cada parâmetro observado, foi possível compor a Matriz $A$ da rede topográfica espacial proposta nesta tese. Para se obter a solução $(3 D)$, esta matriz foi organizada pelas equações de observações para distâncias inclinadas, ângulos verticais zenitais e ângulos horizontais azimutais, sucessivamente, o que 
gerou uma Matriz A com dimensão 90x15. A aplicação prática da rede topográfica espacial pode ser vista detalhadamente na seção 8.2.1. 


\section{Capítulo}

\section{MÉTODOS DE DETERMINAÇÃO DE COORDENADAS ESPACIAIS}

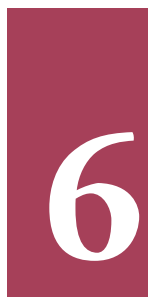

Os métodos de determinações de coordenadas espaciais utilizados no monitoramento geodésico de estruturas, são basicamente os mesmos utilizados nos cálculos topográficos e geodésicos convencionais. As características que os diferenciam estão relacionados às técnicas de medições e modelagens matemáticas empregadas. Dessa forma, apresentam-se os métodos que são objetos de estudo desta tese, com objetivo de verificar quais dentre eles, oferecem o nível de precisão e de acurácia, exigidos em auscultações estruturais (OGUNDARE, 2015).

Dessa forma, os métodos de determinação de coordenadas espaciais podem ser categorizados em métodos com medição de distâncias e métodos sem medição de distâncias. Essa categorização ocorre devido ao fato de haver casos em que não é possível instalar um prisma refletor sobre o ponto em que se deseja determinar as coordenadas. Nesses casos, as determinações são feitas somente em função de medições angulares. Entretanto, na maioria dos casos, principalmente para o monitoramento de estruturas, é possível instalar um prisma refletor no ponto de medição. As determinações das coordenadas espaciais, nestes casos, são feitas em função de medições de ângulos e distâncias.

Outra situação recorrente é a determinação das coordenadas espaciais com superabundância de medições, ou seja, por meio de mais visadas entre as estações de coordenadas conhecidas e o alvo, do que as estritamente necessárias. A solução, nestes casos, é obtida por meio da aplicação de métodos de ajustamento de observações para a determinação das melhores coordenadas possíveis para o ponto observado. A superabundância de dados pode ocorrer tanto para os métodos de determinação de coordenadas com medição de distâncias, quanto para os métodos sem medição de distâncias. Neste capítulo estão apresentadas as formulações matemáticas para ambos os métodos. 
Existem diferentes situações geométricas de determinações de coordenadas espaciais, que devem ser consideradas para o estudo da matéria. A Figura 6.1 ilustra uma medição polar simples.

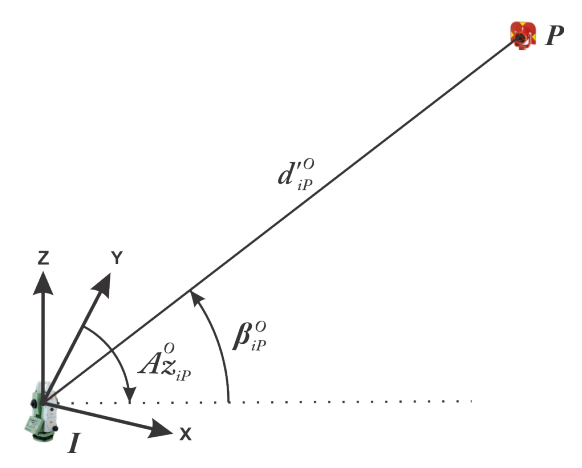

Figura 6.1: Medição polar simples.

Enquanto a Figura 6.2 mostra a melhor situação geométrica possível para uma interseção, porém, pouco provável na prática.

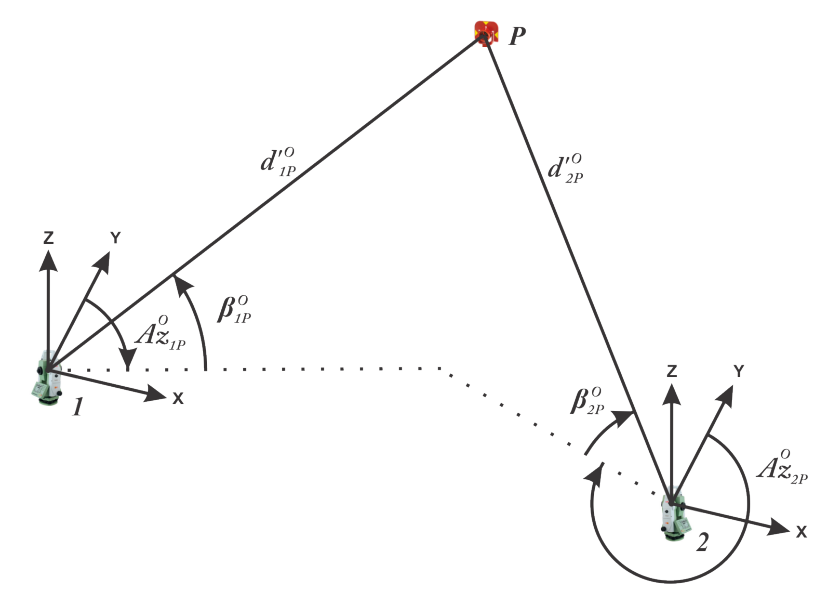

Figura 6.2: Interseção perfeita.

Por fim, a Figura 6.3 mostra como é a situação geométrica na prática.

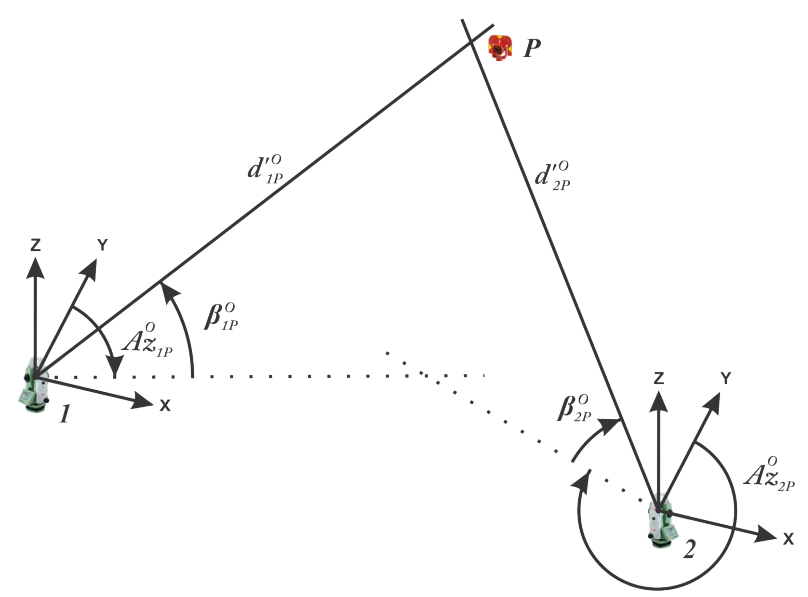

Figura 6.3: Interseção na prática. 
Ressalta-se que, para os métodos que envolvem vários instrumentos de medição, ou seja, $n$ estações totais robóticas, é possível determinar as coordenadas espaciais por meio da média aritmética simples, realizando-se as medições e determinações de coordenadas espaciais, tomadas duas a duas ou tomadas três a três. Para isso deve-se realizar o cálculo de combinações entre as estações envolvidas. Dessa forma, para melhorar o resultado dos valores das coordenadas calculadas para o ponto $P$, pode-se aumentar a quantidade de visadas. Neste caso, as coordenadas do ponto podem ser calculadas a partir de $n$ visadas combinadas gerando $N$ interseções.

O primeiro método apresentado é o polar. Nesta tese, discutem-se, detalhadamente, as variantes do método em função do modelo físico adotado no processo de medição. Em seguida, discutem-se os métodos de determinação de coordenadas espaciais sem medição de distâncias e, finalmente, os métodos com medição de distâncias. Alguns métodos foram aprimorados no Laboratório de Geomática da EESC e outros foram adaptados de propostas apresentadas em artigos científicos.

A partir dos anos 80, com o advento das estações totais, que permitem medir ângulos e distâncias concomitantemente, passou-se a utilizar o método da irradiação topográfica espacial, ou método polar, como uma opção mais simples. A medição dos pontos, neste caso, já não precisa ser dividida em duas etapas, uma vez que o método permite medir, em apenas uma, as coordenadas espaciais $(X, Y, Z)$. Instalações baseadas neste método são encontradas em várias aplicações de monitoramento, principalmente naquelas relacionadas à mineração e à construção civil, como pode ser visto em (WUNDERLICH, 2004). Vários programas aplicativos baseados neste método estão disponíveis no mercado e têm sido utilizados, regularmente, com sucesso.

O método polar, entretanto, por não possuir redundância, permite um controle restrito da qualidade das medições. Por esta razão, tem sido objeto de estudos atuais o emprego de métodos de ajustamento de redes espaciais, nos quais as medições de vários instrumentos de medição, em vários momentos, são combinados para gerarem dados redundantes, permitindo, assim, detectar erros de medições e avaliar a precisão e a confiabilidade dos resultados medidos. A Figura 6.4 mostra um exemplo de instalações para o monitoramento com medição polar simples e com medição polar múltipla. O método de monitoramento, por meio de medições polares múltiplas, não se aplica, naturalmente, a apenas um ponto de medição. Apresenta-se, na sequência, o desenvolvimento matemático para a aplicação do método polar e o desenvolvimento matemático para a aplicação dos métodos multipolares. 


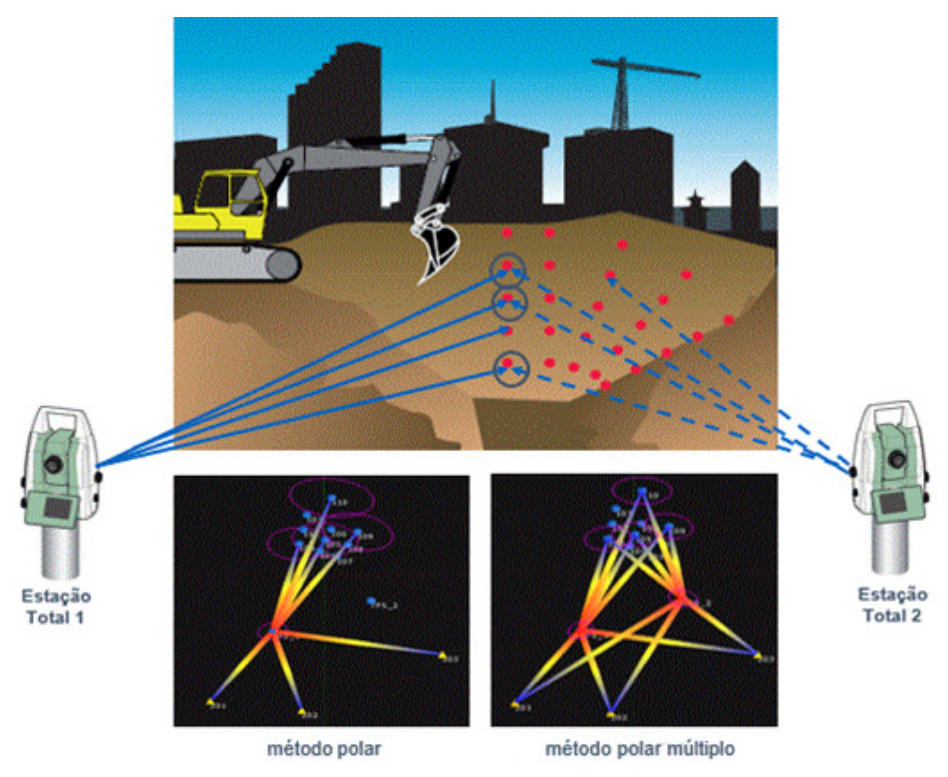

Figura 6.4: Medição polar e multipolar.

\subsection{Método polar}

Método Polar é o método de determinação de coordenadas espaciais, baseado na medição de direções horizontais, ângulos verticais e distâncias inclinadas entre a estação de referência e o ponto a ser medido. Desta forma, de acordo com a Figura 6.5, as coordenadas $\left(X_{P}, Y_{P}, Z_{P}\right)$ do ponto $P$, observadas a partir de um ponto $I$ de coordenadas conhecidas $\left(X_{i}, Y_{i}, Z_{i}\right)$, devidamente orientado a um ponto $R$ de coordenadas $\left(X_{R}, Y_{R}, Z_{R}\right)$, podem ser calculadas aplicando-se as equações (6.1), (6.2) e (6.3), indicadas a seguir:

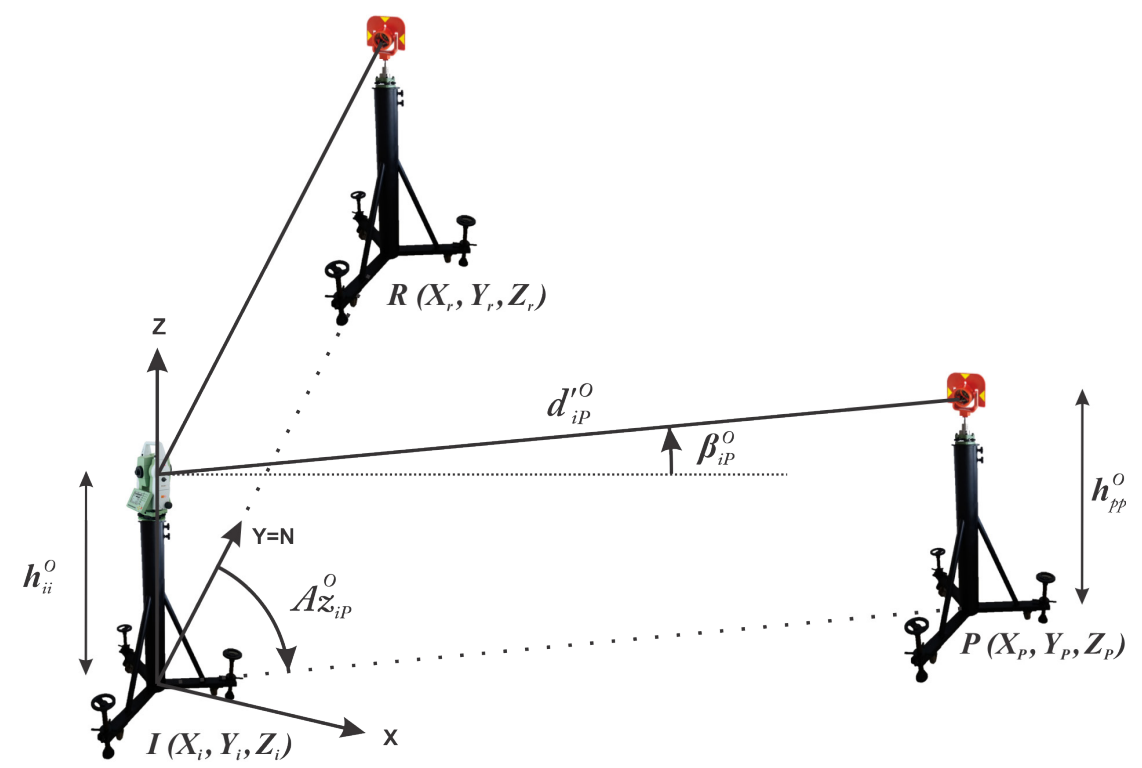

Figura 6.5: Relações geométricas do método polar. 


$$
\begin{aligned}
& X_{P}=X_{i}+d_{i P}^{\prime O} \cdot \cos \left(\beta_{i P}^{O}\right) \cdot \operatorname{sen}\left(A z_{i P}^{O}\right) \\
& Y_{P}=Y_{i}+d_{i P}^{\prime O} \cdot \cos \left(\beta_{i P}^{O}\right) \cdot \cos \left(A z_{i P}^{O}\right) \\
& Z_{P}=Z_{i}+d_{i P}^{\prime O} \cdot \operatorname{sen}\left(\beta_{i P}^{O}\right)+h_{i i}^{O}-h_{p p}^{O}
\end{aligned}
$$

Sendo,

$X_{i}, Y_{i}, Z_{i}$ - coordenadas espaciais conhecidas para o ponto $I$

$X_{r}, Y_{r}, Z_{r}$ - coordenadas espaciais do ponto de orientação $R$

$X_{P}, Y_{P}, Z_{P}$ - coordenadas espaciais calculadas para o ponto $P$

$d_{i P}^{\prime O}$ - distância inclinada observada entre os pontos $I$ e $P$

$\beta_{i P}^{O}$ - ângulo vertical de altura observado entre os pontos $I$ e $P$

$A z_{i P}^{O}$ - ângulo horizontal azimutal observado entre os pontos $I$ e $P$

$h_{i i}^{O}$ - altura do instrumento no ponto $I$

$h_{p p}^{O}$ - altura do prisma no ponto $P$

Para simplificação do modelo matemático, considera-se,

$$
a_{i P}=\cos \left(\beta_{i P}^{O}\right) \cdot \operatorname{sen}\left(A z_{i P}^{O}\right) \quad b_{i P}=\cos \left(\beta_{i P}^{O}\right) \cdot \cos \left(A z_{i P}^{O}\right) \quad c_{i P}=\operatorname{sen}\left(\beta_{i P}^{O}\right)
$$

Nestas condições, as equações (6.1), (6.2) e (6.3) podem ser escritas na forma matricial indicada na sequência.

$$
F=F_{0}+d_{i P}^{\prime O} \cdot A \Leftrightarrow\left[\begin{array}{c}
X_{P} \\
Y_{P} \\
Z_{P}
\end{array}\right]=\left[\begin{array}{c}
X_{i} \\
Y_{i} \\
Z_{i}
\end{array}\right]+d_{i P}^{\prime O} \cdot\left[\begin{array}{c}
a_{i P} \\
b_{i P} \\
c_{i P}
\end{array}\right]
$$

Pela Teoria dos Erros, o erro médio quadrático pode ser calculado. Considerando $F^{\prime T}$, como sendo o vetor das derivadas da função $F$, em relação às observações $d_{i P}^{\prime O}, \beta_{i P}^{O}$ e $A z_{i P}^{O}$, obtém-se:

$$
K_{f f}=F^{\prime T} \cdot K_{l l} \cdot F^{\prime}
$$

Sendo,

$$
K_{f f}=\left[\begin{array}{ccc}
\sigma_{X}^{2} & \sigma_{X Y} & \sigma_{X Z} \\
\sigma_{Y X} & \sigma_{Y}^{2} & \sigma_{Y Z} \\
\sigma_{Z X} & \sigma_{Z Y} & \sigma_{Z}^{2}
\end{array}\right] \text { - matriz de variância-covariância das funções }
$$




$$
\begin{aligned}
K_{l l} & =\left[\begin{array}{ccr}
\sigma_{d_{i p}^{\prime}}^{2} & 0 & 0 \\
0 & \sigma_{\beta_{i p}^{O}}^{2} & \sigma_{\beta_{i p}^{O}, A z_{i p}^{O}} \\
0 & \sigma_{A z_{i p}^{O}, \beta_{i p}^{O}} & \sigma_{A z_{i p}^{O}}^{2}
\end{array}\right] \text { - matriz de variância-covariância das observações } \\
F^{T} & =\left[\begin{array}{lll}
\frac{\partial F}{\partial d_{i P}^{\prime O}} & \frac{\partial F}{\partial \beta_{i P}^{O}} & \frac{\partial F}{\partial A z_{i P}^{O}}
\end{array}\right] \text { - derivadas parciais }
\end{aligned}
$$

As derivadas da função $F$, em relação às observações $d_{i P}^{\prime O}, \beta_{i P}^{O}$ e $A z_{i P}^{O}$ :

$$
\begin{array}{lll}
\frac{\partial X_{P}}{\partial d_{i P}^{\prime O}}=\cos \left(\beta_{i P}^{O}\right) \cdot \operatorname{sen}\left(A z_{i P}^{O}\right) & \frac{\partial Y_{P}}{\partial d_{i P}^{\prime O}}=\cos \left(\beta_{i P}^{O}\right) \cdot \cos \left(A z_{i P}^{O}\right) & \frac{\partial Z_{P}}{\partial d_{i P}^{\prime O}}=\operatorname{sen}\left(\beta_{i P}^{O}\right) \\
\frac{\partial X_{P}}{\partial \beta_{i P}^{O}}=-d_{i P}^{\prime O} \cdot \operatorname{sen}\left(\beta_{i P}^{O}\right) \cdot \operatorname{sen}\left(A z_{i P}^{O}\right) & \frac{\partial Y_{P}}{\partial \beta_{i P}^{O}}=-d_{i P}^{\prime O} \cdot \operatorname{sen}\left(\beta_{i P}^{O}\right) \cdot \cos \left(A z_{i P}^{O}\right) & \frac{\partial Z_{P}}{\partial \beta_{i P}^{O}}=d_{i P}^{\prime O} \cdot \cos \left(\beta_{i P}^{O}\right) \\
\frac{\partial X_{P}}{\partial A z_{i P}^{O}}=d_{i P}^{\prime O} \cdot \cos \left(\beta_{i P}^{O}\right) \cdot \cos \left(A z_{i P}^{O}\right) & \frac{\partial Y_{P}}{\partial A z_{i P}^{O}}=-d_{i P}^{\prime O} \cdot \cos \left(\beta_{i P}^{O}\right) \cdot \operatorname{sen}\left(A z_{i P}^{O}\right) & \frac{\partial Z_{P}}{\partial A z_{i P}^{O}}=0
\end{array}
$$

E seus parâmetros,

$\sigma_{X_{i P}}, \sigma_{Y_{i P}}, \sigma_{Z_{i P}}$ - precisões das coordenadas $\left(X_{i P}, Y_{i P}, Z_{i P}\right)$ do ponto medido

$\sigma_{X Y_{i P}}, \sigma_{X Z_{i P}}, \sigma_{Y Z_{i P}}$ - covariâncias das coordenadas $\left(X_{i P}, Y_{i P}, Z_{i P}\right)$ do ponto medido

$\sigma_{d_{i P}^{\prime O}}, \sigma_{\beta_{i P}^{O}}, \sigma_{A z_{i P}^{O}}$ - precisões das observações em relação ao ponto medido

$\sigma_{\beta_{i P}^{O} A z_{i P}^{O}}$ - covariância entre as observações $\beta_{i P}^{O}$ e $A z_{i P}^{O}$

Ao aplicar as equações acima, obtém-se o terno de coordenadas $\left(X_{P}, Y_{P}, Z_{P}\right)$ do ponto $P$ e suas respectivas precisões. Se forem realizadas $n$ medições polares, as coordenadas ajustadas podem ser calculadas pela média ponderada das coordenadas individuais de cada medição polar. Neste caso, o peso de cada coordenada é dado pela equação (6.7).

$$
P_{X_{P(n)}}=\frac{\sigma_{X_{0}}^{2}}{\sigma_{X_{P(n)}}^{2}} \quad P_{Y_{P(n)}}=\frac{\sigma_{Y_{0}}^{2}}{\sigma_{Y_{P(n)}}^{2}} \quad P_{Z_{P(n)}}=\frac{\sigma_{Z_{0}}^{2}}{\sigma_{Z_{P(n)}}^{2}}
$$

Assim,

$$
\bar{X}_{P}=\frac{P_{X_{P(n)}}^{T} \cdot X_{P}}{e^{T} \cdot P_{X_{P(n)}}} \quad \bar{Y}_{P}=\frac{P_{Y_{P(n)}}^{T} \cdot Y_{P}}{e^{T} \cdot P_{Y_{P(n)}}} \quad \bar{Z}_{P}=\frac{P_{Z_{P(n)}}^{T} \cdot Z_{P}}{e^{T} \cdot P_{Z_{P(n)}}}
$$


Sendo,

$$
\begin{gathered}
\bar{X}_{P}=\left[\begin{array}{c}
X_{P(1)} \\
X_{P(2)} \\
\vdots \\
X_{P(n)}
\end{array}\right] \quad \bar{Y}_{P}=\left[\begin{array}{c}
Y_{P(1)} \\
Y_{P(2)} \\
\vdots \\
Y_{P(n)}
\end{array}\right] \quad \bar{Z}_{P}=\left[\begin{array}{c}
Z_{P(1)} \\
Z_{P(2)} \\
\vdots \\
Z_{P(n)}
\end{array}\right] \\
P_{X_{P}}=\left[\begin{array}{c}
P_{X_{P(1)}} \\
P_{X_{P(2)}} \\
\vdots \\
P_{X_{P(n)}}
\end{array}\right] \quad P_{Y_{P}}=\left[\begin{array}{c}
P_{Y_{P(1)}} \\
P_{Y_{P(2)}} \\
\vdots \\
P_{Y_{P(n)}}
\end{array}\right] \quad P_{Z_{P}}=\left[\begin{array}{c}
P_{Z_{P(1)}} \\
P_{Z_{P(2)}} \\
\vdots \\
P_{Z_{P(n)}}
\end{array}\right] \quad e=\left[\begin{array}{c}
1 \\
1 \\
\vdots \\
1
\end{array}\right]
\end{gathered}
$$

$\bar{X}_{P}, \bar{Y}_{P}, \bar{Z}_{P}$ - coordenadas ajustadas do ponto $P$

$P_{X_{P}}, P_{Y_{P}}, P_{Z_{P}}$ - vetores dos pesos

As precisões $\sigma_{\bar{X}_{P}}^{2}, \sigma_{\bar{Y}_{P}}^{2}$ e $\sigma_{\bar{Z}_{P}}^{2}$ das médias são calculadas de acordo com suas respectivas equações, como podem ser vistas em (6.9).

$$
\sigma_{\bar{X}_{P}}^{2}=\frac{\sigma_{X_{0}}^{2}}{e^{T} \cdot P_{X_{P}}} \quad \sigma_{\bar{Y}_{P}}^{2}=\frac{\sigma_{Y_{0}}^{2}}{e^{T} \cdot P_{Y_{P}}} \quad \sigma_{\bar{Z}_{P}}^{2}=\frac{\sigma_{Z_{0}}^{2}}{e^{T} \cdot P_{Z_{P}}}
$$

\subsection{Métodos multipolares sem medições de distâncias}

\subsubsection{Método de interseção espacial trigonométrico}

O Método de Interseção Espacial Trigonométrico foi um dos primeiros métodos desenvolvido para a determinação de coordenadas espaciais por meio de medições angulares, sem a intervenção da medição de distâncias. Tal método é dividido em duas etapas, na primeira, determinam-se as coordenadas planas do alvo, a partir do cálculo de uma interseção plana; e, na segunda, determina-se a altura do alvo, por meio das fórmulas do nivelamento trigonométrico. A coordenada plana do alvo é calculada por meio da medição dos ângulos horizontais entre duas estações de referência e o alvo, ressaltando que não é utilizada nenhuma medição de distância. Após da determinação das coordenadas planas do alvo e da medição do ângulo vertical zenital ou de altura de uma das linhas de visada, calcula-se a coordenada $Z$ do alvo, como pode ser visto na Figura 6.6. 


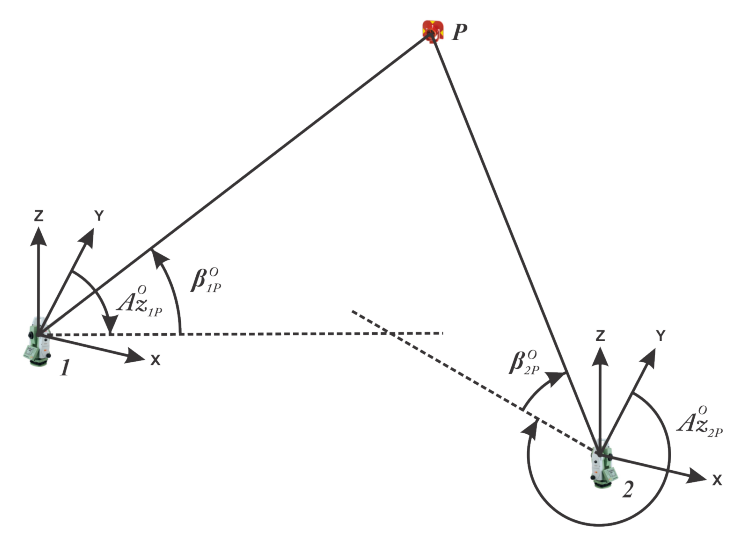

Figura 6.6: Representação geométrica do método de interseção plana.

A inconveniência do método reside no fato de que um erro, cometido na leitura do ângulo vertical, não influencia no resultado das coordenadas planas e, um erro, cometido na leitura de um ângulo horizontal, não altera o valor da coordenada $Z$, conforme exibido na Figura 6.7.

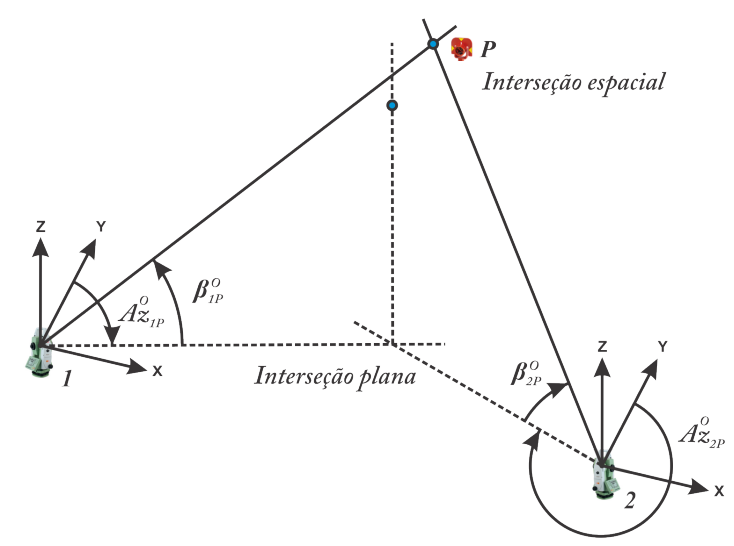

Figura 6.7: Representação da discrepância do método de interseção espacial trigonométrico.

Para determinar as coordenadas de um ponto $P$, por interseção espacial trigonométrica, é necessário conhecer as coordenadas de duas estações de referência $\left(X_{i}, Y_{i}, Z_{i}\right)$, sendo $i=1 \mathrm{e}$ 2 , além das observações dos azimutes $A z_{i P}^{O}$ e dos ângulos verticais de altura $\beta_{i P}^{O}$ em relação ao alvo. As coordenadas planimétricas podem ser calculadas, aplicando-se o Método de Interseção a Vante, conforme descrito na sequência. Para efeito de apresentação do modelo, denota-se por $\left(X_{1}, Y_{1}, Z_{1}\right)$ as coordenadas da estação de referência 1 e por $\left(X_{2}, Y_{2}, Z_{2}\right)$ as coordenadas da estação de referência 2. Com isso, calculam-se as coordenadas $\left(X_{P}, Y_{P}, Z_{P}\right)$ do ponto alvo $P$.

De posse das observações dos azimutes $A z_{1 P}^{O}$ e $A z_{2 P}^{O}$; e dos ângulos verticais de altura $\beta_{1 P}^{O}$ e $\beta_{2 P}^{O}$ das linhas de visadas $1 P$ e $2 P$, as coordenadas planimétricas do ponto $P$ podem ser calculadas pelas seguintes equações.

Se,

$$
\left|\operatorname{tg}\left(A z_{2 P}^{O}\right)\right|<\left|\operatorname{tg}\left(A z_{1 P}^{O}\right)\right|
$$


Então,

$$
\begin{gathered}
Y_{P}=Y_{1}+\frac{\left(X_{2}-X_{1}\right)-\left(Y_{2}-Y_{1}\right) \cdot \operatorname{tg}\left(A z_{2 P}^{O}\right)}{\operatorname{tg}\left(A z_{1 P}^{O}\right)-\operatorname{tg}\left(A z_{2 P}^{O}\right)} \\
X_{P}=X_{2}+\left(Y_{P}-Y_{2}\right) \cdot \operatorname{tg}\left(A z_{2 P}^{O}\right)
\end{gathered}
$$

Se,

$$
\left|\operatorname{tg}\left(A z_{1 P}^{O}\right)\right|<\left|\operatorname{tg}\left(A z_{2 P}^{O}\right)\right|
$$

Então,

$$
\begin{gathered}
Y_{P}=Y_{2}+\frac{\left(X_{1}-X_{2}\right)-\left(Y_{1}-Y_{2}\right) \cdot \operatorname{tg}\left(A z_{1 P}^{O}\right)}{\operatorname{tg}\left(A z_{2 P}^{O}\right)-\operatorname{tg}\left(A z_{1 P}^{O}\right)} \\
X_{P}=X_{1}+\left(Y_{P}-Y_{1}\right) \cdot \operatorname{tg}\left(A z_{1 P}^{O}\right)
\end{gathered}
$$

Têm-se assim as coordenadas $X_{P}$ e $Y_{P}$ e, por conseguinte, as distâncias horizontais $d_{1 P}$ e $d_{2 P}$, por meio das quais pode ser realizado o cálculo da coordenada $Z_{i P}$ do ponto $P$.

$$
\begin{aligned}
& Z_{1 P}=Z_{1}+\operatorname{tg}\left(\beta_{1 P}^{O}\right) \cdot \sqrt{\left(X_{P}-X_{1}\right)^{2}+\left(Y_{P}-Y_{1}\right)^{2}} \\
& Z_{2 P}=Z_{2}+\operatorname{tg}\left(\beta_{2 P}^{O}\right) \cdot \sqrt{\left(X_{P}-X_{2}\right)^{2}+\left(Y_{P}-Y_{2}\right)^{2}}
\end{aligned}
$$

A coordenada $Z_{P}$ final do ponto pode ser calculada pela média aritmética das coordenadas $Z_{1 P}$ e $Z_{2 P}$, oriunda de cada linha de visada. Assim,

$$
\bar{Z}_{P}=\frac{Z_{1 P}+Z_{2 P}}{2}
$$

Têm-se, assim, as coordenadas $\left(X_{P}, Y_{P}, Z_{P}\right)$ do ponto $P$.

Sendo,

$X_{i}, Y_{i}, Z_{i}$ - coordenadas espaciais conhecidas para as estações de referências $I$

$X_{P}, Y_{P}, Z_{P}$ - coordenadas espaciais calculadas para o ponto alvo $P$

$d_{i P}^{C}$ - distância horizontal calculada entre os pontos $I$ e $P$

$\beta_{i P}^{O}$ - ângulo vertical de altura observado entre os pontos $I$ e $P$

$A z_{i P}^{O}$ - ângulo horizontal azimutal observado entre os pontos $I$ e $P$ 
Para os casos em que se utilizam três ou mais instrumentos de medição, ou seja $n>2$, podem-se realizar os cálculos, considerando combinações aleatórias, tomadas duas a duas. E, por meio dessas combinações, extrair a média aritmética $\left(\bar{X}_{1,2,3, \cdots, n, P}, \bar{Y}_{1,2,3, \cdots, n, P}, \bar{Z}_{1,2,3, \cdots, n, P}\right)$, para o terno de coordenadas do ponto $P$.

\subsubsection{Método do ponto médio da interseção espacial}

Para superar o problema da inconsistência do método da interseção espacial trigonométrica, foram desenvolvidas por Allan (1988), Allan (1990) e Allan (1996) outras técnicas de interseção e, a solução adotada consiste basicamente em considerar que o ponto $P$, a ser determinado, encontra-se na mediana da perpendicular comum entre as duas semirretas, que partem das estações 1 e 2 , em direção ao ponto $P$. As semirretas $1 P$ e $2 P$ são geradas pelas linhas de visadas de duas estações totais, estacionadas sobre o ponto 1 e 2 , visando o ponto $P$. Para a orientação das linhas de visadas, pode-se adotar o princípio das visadas mútuas entre os dois instrumentos ou utilizar um ou mais pontos de controle com coordenadas conhecidas, determinadas, previamente, por meio de um levantamento topográfico adequado. Os desenvolvimentos matemáticos apresentados a seguir consideram que os instrumentos estão orientados em relação a um sistema de coordenadas espaciais $(X, Y, Z)$ predefinido. Nestas condições, considera-se que os dois instrumentos topográficos, instalados e nivelados sobre os pontos 1 e 2, permitem medir os ângulos horizontais e verticais de cada semirreta, em relação ao sistema de coordenadas $(X, Y, Z)$.

Uma rotina de cálculo disponível na literatura foi apresentada por Allan (1990), a qual consiste em utilizar as equações do método polar e as relações geométricas da interseção espacial para calcular as distâncias inclinadas $d_{1 P}^{\prime C}$ e $d_{2 P}^{\prime C}$ para, em seguida, calcular as coordenadas dos pontos $P_{1 P}\left(X_{1 P}, Y_{1 P}, Z_{1 P}\right)$ e $P_{2 P}\left(X_{2 P}, Y_{2 P}, Z_{2 P}\right)$ e, por fim, as coordenadas $\left(X_{1,2, P}, Y_{1,2, P}, Z_{1,2, P}\right)$ compensadas do ponto $P$, como mostra a Figura 6.8.

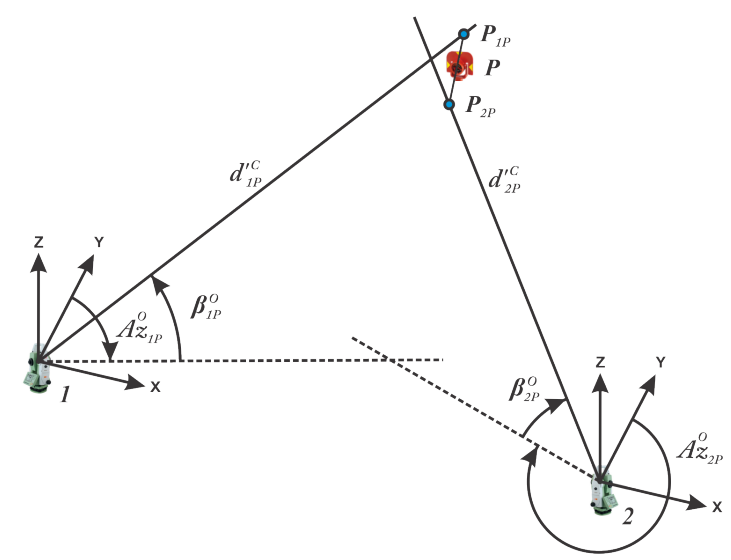

Figura 6.8: Relações geométricas do ponto médio da interseção espacial. 
Dessa forma, de acordo com as equações (6.17) e (6.18), têm-se:

$$
\begin{array}{ll}
a_{1 P}=\cos \left(\beta_{1 P}^{O}\right) \cdot \operatorname{sen}\left(A z_{1 P}^{O}\right) & b_{1 P}=\cos \left(\beta_{1 P}^{O}\right) \cdot \cos \left(A z_{1 P}^{O}\right) \quad c_{1 P}=\operatorname{sen}\left(\beta_{1 P}^{O}\right) \\
a_{2 P}=\cos \left(\beta_{2 P}^{O}\right) \cdot \operatorname{sen}\left(A z_{2 P}^{O}\right) & b_{2 P}=\cos \left(\beta_{2 P}^{O}\right) \cdot \cos \left(A z_{2 P}^{O}\right) \quad c_{2 P}=\operatorname{sen}\left(\beta_{2 P}^{O}\right)
\end{array}
$$

Considerando que as coordenadas dos pontos 1 e 2 são conhecidas e iguais a $\left(X_{1}, Y_{1}\right)$ e $\left(X_{2}, Y_{2}\right)$, e que as distâncias inclinadas calculadas entre os pontos $1 P$ e $2 P$ são iguais a $d_{1 P}^{\prime C}$ e $d_{2 P}^{\prime C}$, respectivamente, têm-se:

$$
\begin{aligned}
& X_{1 P}=X_{1}+a_{1 P} \cdot d_{1 P}^{\prime C} \quad Y_{1 P}=Y_{1}+b_{1 P} \cdot d_{1 P}^{\prime C} \quad Z_{1 P}=Z_{1}+c_{1 P} \cdot d_{1 P}^{\prime C} \\
& X_{2 P}=X_{2}+a_{2 P} \cdot d_{2 P}^{\prime C} \quad Y_{2 P}=Y_{2}+b_{2 P} \cdot d_{2 P}^{\prime C} \quad Z_{2 P}=Z_{2}+c_{2 P} \cdot d_{2 P}^{\prime C}
\end{aligned}
$$

Dessa forma, calculam-se as coordenadas dos pontos $P_{1}$ e $P_{2}$.

Com isso, mostra-se que,

$$
\cos \gamma=a_{1 P} \cdot a_{2 P}+b_{1 P} \cdot b_{2 P}+c_{1 P} \cdot c_{2 P}
$$

Sendo,

$\gamma$ - ângulo entre as projeções e as linhas de visada

$$
\operatorname{sen}^{2} \gamma=1-\cos ^{2} \gamma
$$

Ainda pela Figura 6.9, a projeção de 12 sobre $1 P$ é dada por:
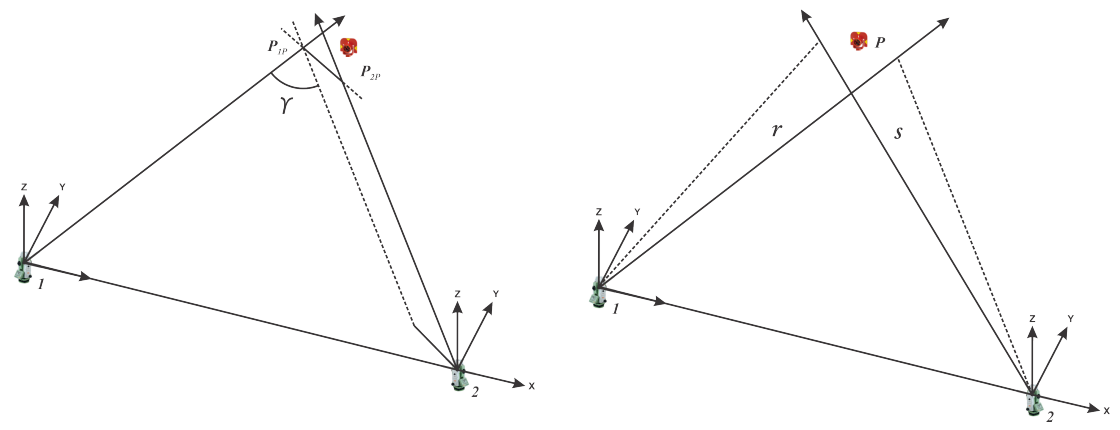

Figura 6.9: Pontos $P_{1}$ e $P_{2}$ e projeções $r$ e $s$ da interseção espacial pelo ponto médio.

$$
r=a_{1 P} \cdot \Delta X_{12}+b_{1 P} \cdot \Delta Y_{12}+c_{1 P} \cdot \Delta Z_{1 P}
$$


A projeção de 12 sobre $2 P$ é dada por:

$$
s=a_{2 P} \cdot \Delta X_{12}+b_{2 P} \cdot \Delta Y_{12}+c_{2 P} \cdot \Delta Z_{12}
$$

De onde se obtêm:

$$
\begin{aligned}
& d_{1 P}^{\prime C}=\frac{(r-s \cdot \cos \gamma)}{\operatorname{sen}^{2} \gamma} \\
& d_{2 P}^{\prime C}=d_{1 P}^{\prime C} \cdot \cos \gamma-s
\end{aligned}
$$

Para evitar valores negativos e o uso de módulos no desenvolvimento matemático e computacional, a variação $\Delta X_{12}$ entre as coordenadas deve ser a mesma para $r$ e $s$; e, para o cálculo de $d_{2 P}^{\prime C}$, deve-se realizar $\left(d_{1 P}^{\prime C} \cdot \cos \gamma-s\right)$ e não $\left(s-d_{1 P}^{\prime C} \cdot \cos \gamma\right)$.

Substituindo os valores de $d_{1 P}^{\prime C}$ e $d_{2 P}^{\prime C}$, nas equações (6.19) e (6.20), obtêm-se os valores das coordenadas dos pontos $P_{1}$ e $P_{2}$. As coordenadas ajustadas do ponto $P$ são calculadas pela média aritmética das coordenadas individuais, calculadas em cada linha de visada, conforme as equações (6.27). Assim,

$$
\bar{X}_{1,2, P}=\frac{X_{1 P}+X_{2 P}}{2} \quad \bar{Y}_{1,2, P}=\frac{Y_{1 P}+Y_{2 P}}{2} \quad \bar{Z}_{1,2, P}=\frac{Z_{1 P}+Z_{2 P}}{2}
$$

Para os casos em que se utilizam três ou mais instrumentos de medição, ou seja $n>2$, podem-se realizar os cálculos, considerando combinações aleatórias, tomadas duas a duas. E, por meio dessas combinações, extrair a média aritmética $\left(\bar{X}_{1,2,3, \cdots, n, P}, \bar{Y}_{1,2,3, \cdots, n, P}, \bar{Z}_{1,2,3, \cdots, n, P}\right)$, para o terno de coordenadas do ponto $P$.

\subsubsection{Método das distâncias mínimas}

No que concerne à situação geométrica do problema da interseção espacial sem medição de distâncias, foi desenvolvido no Laboratório de Geomática uma metodologia de medição e de cálculos matemáticos, baseados na minimização do somatório das distâncias entre os supostos pontos de interseção de cada linha de visada e o ponto ajustado. Igualmente aos casos anteriores, o método consiste na determinação das coordenadas espaciais do alvo, em função das coordenadas das estações de referência conhecidas, dos azimutes dos alinhamentos e dos ângulos verticais de altura ou zenital de cada linha de visada. Assim, a relação geométrica deste método é indicada na Figura 6.10 . 


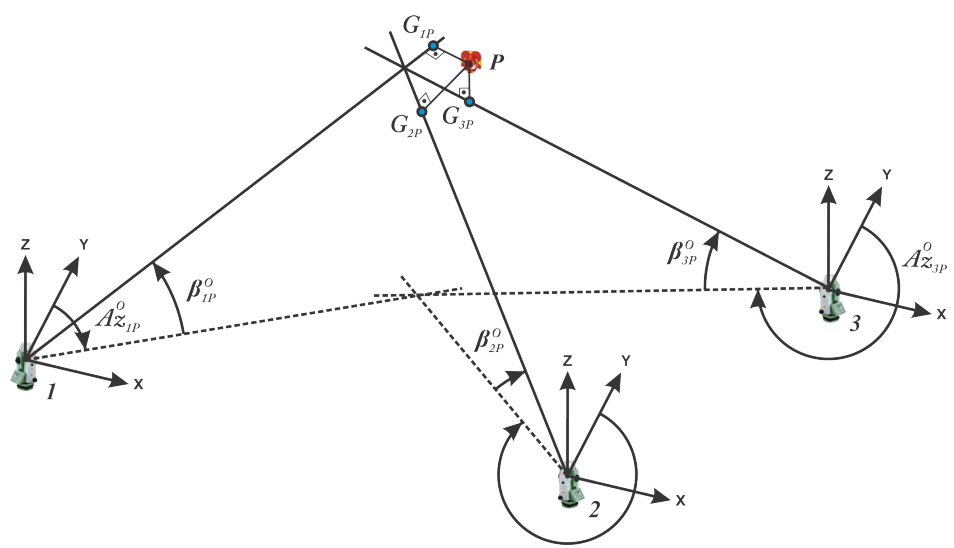

Figura 6.10: Geometria da interseção espacial pelas distâncias mínimas.

O método considera que, quando o somatório das distâncias do ponto $P$ às retas definidas pelas visadas for mínimo, obtém-se a melhor coordenada para o ponto. Dessa forma, para que a minimização seja possível, é necessário, primeiramente, estabelecer as equações das retas definidas pelas linhas de visadas de cada instrumento dirigido ao alvo $P$. As retas possuem como origem o centro óptico de cada instrumento topográfico estacionado nos pontos de coordenadas conhecidas $\left(X_{C O_{i}}, Y_{C O_{i}}, Z_{C O_{i}}\right)$. A direção de cada reta é dada pelo versor $\vec{v}_{i P}$, definido em função dos seus cossenos diretores, baseados nos azimutes e nos ângulos verticais observados. Assim,

$$
\vec{v}_{i P}=\left(a_{i P}, b_{i P}, c_{i P}\right)
$$

Os parâmetros do versor (6.28) são definidos pelas equações (6.4), repetidas em (6.29).

$$
a_{i P}=\cos \left(\beta_{i P}^{O}\right) \cdot \operatorname{sen}\left(A z_{i P}^{O}\right) \quad b_{i P}=\cos \left(\beta_{i P}^{O}\right) \cdot \cos \left(A z_{i P}^{O}\right) \quad c_{i P}=\operatorname{sen}\left(\beta_{i P}^{O}\right)
$$

A reta genérica $r_{i P}$ tem como origem o centro óptico de cada instrumento e a direção do versor $\vec{v}_{i p}$, logo:

$$
r_{i P}=C O_{i}+t_{i P} \cdot \vec{v}_{i P}
$$

Sendo,

$\mathrm{CO}_{i}$ - centro óptico do instrumento, de coordenadas $\left(X_{C O_{i}}, Y_{C O_{i}}, Z_{C O_{i}}\right)$

$t_{i P}$ - parâmetro da reta genérica

$\vec{v}_{i P}$ - versor da reta $r_{i P}$

Os pontos sobre as retas genéricas $r_{i}$, em que uma perpendicular com origem no alvo $P$ intercepta a referida reta, são denominados, genericamente, de $G_{i}\left(t_{i}\right)$. Nota-se que este ponto é único, uma vez que ele representa o ponto, cuja distância em relção ao alvo $P$ é mínima. Ele 
é definido a partir das coordenadas do centro óptico $C O_{i}$ de cada instrumento e do versor da reta determinada pelos cossenos diretores dos azimutes e dos ângulos verticais, lidos em cada instrumento.

Assim, as coordenadas do ponto genérico $G_{i}\left(t_{i P}\right)$ são dadas por,

$$
X G_{i}=X_{C O_{i}}+t_{i P} \cdot a_{i P} \quad Y G_{i}=Y_{C O_{i}}+t_{i P} \cdot b_{i P} \quad Z G_{i}=Z_{C O_{i}}+t_{i P} \cdot c_{i P}
$$

Sendo,

$G_{i}\left(t_{i P}\right)$ - ponto genérico em função do parâmetro $t_{i P}$

$X G_{i}, Y G_{i}, Z G_{i}$ - coordenadas do ponto genérico $G_{i}$, sobre a reta $r_{i P}$

A distância entre o ponto $P$ e a reta, definida pela linha de visada, pode ser calculada pela equação da distância entre dois pontos. Assim,

$$
d_{i P}^{\prime C}=\sqrt{\left[X_{p}-\left(X_{C O_{i}}+t_{i P} \cdot a_{i P}\right)\right]^{2}+\left[Y_{p}-\left(Y_{C O_{i}}+t_{i P} \cdot b_{i P}\right)\right]^{2}+\left[Z_{p}-\left(Z_{C O_{i}}+t_{i P} \cdot c_{i P}\right)\right]^{2}}
$$

Sendo,

$i$ - número de estações envolvidas no processo de medição, sendo $i=1,2,3, \cdots, n$

A condição de ajustamento é que o somatório de todas as distâncias seja mínimo, ou seja, a função abaixo deve ser minimizada para se obter as coordenadas ajustadas do ponto $P$.

$$
\Sigma d_{i P}^{\prime}=\Sigma \sqrt{\left[X_{P}-\left(X_{C O_{i}}+t_{i P} \cdot a_{i P}\right)\right]^{2}+\left[Y_{P}-\left(Y_{C O_{i}}+t_{i P} \cdot b_{i P}\right)\right]^{2}+\left[Z_{P}-\left(Z_{C O_{i}}+t_{i P} \cdot c_{i P}\right)\right]^{2}}
$$

Ao elevar ao quadrado ambos os membros na equação (6.34) elimina-se a raiz do segundo membro e, assim,

$$
\Sigma{d_{i P}^{\prime}}^{2}=\Sigma\left[X_{P}-\left(X_{C O_{i}}+t_{i P} \cdot a_{i P}\right)\right]^{2}+\left[Y_{P}-\left(Y_{C O_{i}}+t_{i P} \cdot b_{i P}\right)\right]^{2}+\left[Z_{P}-\left(Z_{C O_{i}}+t_{i P} \cdot c_{i P}\right)\right]^{2}
$$

Efetuando-se as operações indicadas em cada parcela do segundo membro da equação (6.34), e denominando o primeiro termo de $M X$, o segundo de $M Y$ e o terceiro de $M Z$, têm-se:

$$
M X=\Sigma\left[\left(X_{P}\right)^{2}-2 \cdot\left(X_{P}\right) \cdot\left(X_{C O_{i}}\right)-2 \cdot\left(X_{P}\right) \cdot a_{i P} \cdot t_{i P}+\left(X_{C O_{i}}\right)^{2}+2 \cdot\left(X_{C O_{i}}\right) \cdot a_{i P} \cdot t_{i P}+\left(a_{i P}\right)^{2} \cdot\left(t_{i P}\right)^{2}\right]
$$

$$
M Y=\Sigma\left[\left(Y_{P}\right)^{2}-2 \cdot\left(Y_{P}\right) \cdot\left(Y_{C O_{i}}\right)-2 \cdot\left(Y_{P}\right) \cdot b_{i P} \cdot t_{i P}+\left(Y_{C O_{i}}\right)^{2}+2 \cdot\left(Y_{C O_{i}}\right) \cdot b_{i P} \cdot t_{i P}+\left(b_{i P}\right)^{2} \cdot\left(t_{i P}\right)^{2}\right]
$$


$M Z=\Sigma\left[\left(Z_{P}\right)^{2}-2 \cdot\left(Z_{P}\right) \cdot\left(Z_{C O_{i}}\right)-2 \cdot\left(Z_{P}\right) \cdot c_{i P} \cdot t_{i P}+\left(Z_{C O_{i}}\right)^{2}+2 \cdot\left(Z_{C O_{i}}\right) \cdot c_{i P} \cdot t_{i P}+\left(c_{i P}\right)^{2} \cdot\left(t_{i P}\right)^{2}\right]$

Assim,

$$
\sum_{1}^{n}\left(d_{i}^{2}\right)=M X+M Y+M Z
$$

Onde, os elementos conhecidos das equações são:

$X_{C O_{i}}, Y_{C O_{i}}, Z_{C O_{i}}$ - coordenadas do centro óptico dos instrumentos

$a_{i P}, b_{i P}, c_{i P}$ - parâmetros dos versores diretores das linhas de visada

E as incógnitas são:

$X_{P}, Y_{P}, Z_{P}$ - coordenadas do alvo $P$

$t_{i P}$ - parâmetros das retas definidas pelas linhas de visada, com $i=1,2,3, \cdots, n$

Para a minimização do somatório das distâncias é necessário calcular as derivadas parciais da função (6.39), onde $F$ é o somatório das distâncias.

$$
F=F\left(X_{P}, Y_{P}, Z_{P}, t_{1 P}, t_{2 P}, t_{3 P}, \cdots, t_{n P}\right)
$$

Derivando a equação (6.39) em função de $X_{P}$, tem-se:

$$
\frac{\partial F}{\partial X_{P}}=2 \cdot n \cdot\left(X_{P}\right)-2 \cdot \Sigma_{1}^{n} X_{C O_{i}}-2 \cdot \Sigma_{1}^{n}\left(a_{i P} \cdot t_{i P}\right)
$$

Desenvolvendo os somatórios, tem-se:

$\frac{\partial F}{\partial X_{P}}=2 \cdot n \cdot\left(X_{P}\right)-2 \cdot\left[\left(X_{C O_{1}}, X_{C O_{2}}+\cdots+X_{C O_{n}}\right)\right]-2 \cdot\left[\left(a_{1 P} \cdot t_{1 P}\right)+\left(a_{2 P} \cdot t_{2 P}\right)+\cdots+\left(a_{n P} \cdot t_{n P}\right)\right]$

Igualando a zero, tem-se:

$2 \cdot n \cdot\left(X_{P}\right)-2 \cdot\left[\left(X_{C O_{1}}, X_{C O_{2}}+\cdots+X_{C O_{n}}\right)\right]-2 \cdot\left[\left(a_{1 P} \cdot t_{1 P}\right)+\left(a_{2 P} \cdot t_{2 P}\right)+\cdots+\left(a_{n P} \cdot t_{n P}\right)\right]=0$

Derivando a equação (6.39) em função de $Y_{P}$, tem-se:

$$
\frac{\partial F}{\partial Y_{P}}=2 \cdot n \cdot\left(Y_{P}\right)-2 \cdot \Sigma_{1}^{n} Y_{C O_{i}}-2 \cdot \Sigma_{1}^{n}\left(b_{i P} \cdot t_{i P}\right)
$$

Desenvolvendo os somatórios, tem-se:

$$
\frac{\partial F}{\partial Y_{P}}=2 \cdot n \cdot\left(Y_{P}\right)-2 \cdot\left[\left(Y_{C O_{1}}, Y_{C O_{2}}+\cdots+Y_{C O_{n}}\right)\right]-2 \cdot\left[\left(b_{1 P} \cdot t_{1 P}\right)+\left(b_{2 P} \cdot t_{2 P}\right)+\cdots+\left(b_{n P} \cdot t_{n P}\right)\right]
$$


Igualando a zero, tem-se:

$2 \cdot n \cdot\left(Y_{P}\right)-2 \cdot\left[\left(Y_{C_{1}}, Y_{C O_{2}}+\cdots+Y_{C O_{n}}\right)\right]-2 \cdot\left[\left(b_{1 P} \cdot t_{1 P}\right)+\left(b_{2 P} \cdot t_{2 P}\right)+\cdots+\left(b_{n P} \cdot t_{n P}\right)\right]=0$

Derivando a equação (6.39) em função de $Z_{P}$, tem-se:

$$
\frac{\partial F}{\partial Z_{P}}=2 \cdot n \cdot\left(Z_{P}\right)-2 \cdot \Sigma_{1}^{n} Z_{C O_{i}}-2 \cdot \Sigma_{1}^{n}\left(c_{i P} \cdot t_{i P}\right)
$$

Desenvolvendo os somatórios, tem-se:

$\frac{\partial F}{\partial Z_{P}}=2 \cdot n \cdot\left(Z_{P}\right)-2 \cdot\left[\left(Z_{C O_{1}}, Z_{C O_{2}}+\cdots+Z_{C O_{n}}\right)\right]-2 \cdot\left[\left(c_{1 P} \cdot t_{1 P}\right)+\left(c_{2 P} \cdot t_{2 P}\right)+\cdots+\left(c_{n P} \cdot t_{n P}\right)\right]$

Igualando a zero, tem-se:

$2 \cdot n \cdot\left(Z_{P}\right)-2 \cdot\left[\left(Z_{C O_{1}}, Z_{C O_{2}}+\cdots+Z_{C O_{n}}\right)\right]-2 \cdot\left[\left(c_{1 P} \cdot t_{1 P}\right)+\left(c_{2 P} \cdot t_{2 P}\right)+\cdots+\left(c_{n P} \cdot t_{n P}\right)\right]=0$

Derivando a equação (6.39) em função de $t_{1 P}$, tem-se:

$$
\begin{aligned}
\frac{\partial F}{\partial t_{1 P}}=-2 & \cdot a_{1 P} \cdot\left(X_{P}\right)-2 \cdot b_{1 P} \cdot\left(Y_{P}\right)-2 \cdot c_{1 P} \cdot\left(Z_{P}\right)+2 \cdot a_{1 P} \cdot\left(X_{C O_{1}}\right)+2 \cdot b_{1 P} \cdot\left(Y_{C O_{1}}\right)+ \\
& +2 \cdot c_{1 P} \cdot\left(Z_{C O_{1}}\right)+2 \cdot\left(a_{1 P}\right)^{2} \cdot t_{1 P}+2 \cdot\left(b_{1 P}\right)^{2} \cdot t_{1 P}+2 \cdot\left(c_{1 P}\right)^{2} \cdot t_{1 P}
\end{aligned}
$$

Igualando a zero, tem-se:

$$
\begin{gathered}
-2 \cdot a_{1 P} \cdot\left(X_{P}\right)-2 \cdot b_{1 P} \cdot\left(Y_{P}\right)-2 \cdot c_{1 P} \cdot\left(Z_{P}\right)+2 \cdot a_{1 P} \cdot\left(X_{C O_{1}}\right)+2 \cdot b_{1 P} \cdot\left(Y_{C O_{1}}\right)+ \\
+2 \cdot c_{1 P} \cdot\left(Z_{C O_{1}}\right)+2 \cdot\left(a_{1 P}\right)^{2} \cdot t_{1 P}+2 \cdot\left(b_{1 P}\right)^{2} \cdot t_{1 P}+2 \cdot\left(c_{1 P}\right)^{2} \cdot t_{1 P}=0
\end{gathered}
$$

Derivando a equação (6.39) em função de $t_{2 P}$, tem-se:

$$
\begin{aligned}
\frac{\partial F}{\partial t_{2 P}}=-2 & \cdot a_{2 P} \cdot\left(X_{P}\right)-2 \cdot b_{2 P} \cdot\left(Y_{P}\right)-2 \cdot c_{2 P} \cdot\left(Z_{P}\right)+2 \cdot a_{2 P} \cdot\left(X_{C_{2}}\right)+2 \cdot b_{2 P} \cdot\left(Y_{C_{2}}\right)+ \\
& +2 \cdot c_{2} \cdot\left(Z_{\mathrm{CO}_{2}}\right)+2 \cdot\left(a_{2 P}\right)^{2} \cdot t_{2 P}+2 \cdot\left(b_{2 P}\right)^{2} \cdot t_{2 P}+2 \cdot\left(c_{2 P}\right)^{2} \cdot t_{2 P P}
\end{aligned}
$$


Igualando a zero, tem-se:

$$
\begin{gathered}
-2 \cdot a_{2 P} \cdot\left(X_{P}\right)-2 \cdot b_{2 P} \cdot\left(Y_{P}\right)-2 \cdot c_{2 P} \cdot\left(Z_{P}\right)+2 \cdot a_{2 P} \cdot\left(X_{C O_{2}}\right)+2 \cdot b_{2 P} \cdot\left(Y_{C O_{2}}\right)+ \\
+2 \cdot c_{2 P} \cdot\left(Z_{C_{2}}\right)+2 \cdot\left(a_{2 P}\right)^{2} \cdot t_{2 P}+2 \cdot\left(b_{2 P}\right)^{2} \cdot t_{2 P}+2 \cdot\left(c_{2 P}\right)^{2} \cdot t_{2 P}=0
\end{gathered}
$$

Derivando a equação (6.39) em função de $t_{3 P}$, tem-se:

$$
\begin{aligned}
\frac{\partial F}{\partial t_{3 P}}=-2 & \cdot a_{3 P} \cdot\left(X_{P}\right)-2 \cdot b_{3 P} \cdot\left(Y_{P}\right)-2 \cdot c_{3 P} \cdot\left(Z_{P}\right)+2 \cdot a_{3 P} \cdot\left(X_{C_{3}}\right)+2 \cdot b_{3 P} \cdot\left(Y_{C_{3}}\right)+ \\
& +2 \cdot c_{3 P} \cdot\left(Z_{\mathrm{CO}_{3}}\right)+2 \cdot\left(a_{3 P}\right)^{2} \cdot t_{3 P}+2 \cdot\left(b_{3 P}\right)^{2} \cdot t_{3 P}+2 \cdot\left(c_{3 P}\right)^{2} \cdot t_{3 P}
\end{aligned}
$$

Igualando a zero, tem-se:

$$
\begin{gathered}
-2 \cdot a_{3 P} \cdot\left(X_{P}\right)-2 \cdot b_{3 P} \cdot\left(Y_{P}\right)-2 \cdot c_{3 P} \cdot\left(Z_{P}\right)+2 \cdot a_{3 P} \cdot\left(X_{C_{3}}\right)+2 \cdot b_{3 P} \cdot\left(Y_{C_{3}}\right)+ \\
\quad+2 \cdot c_{3 P} \cdot\left(Z_{C O_{3}}\right)+2 \cdot\left(a_{3 P}\right)^{2} \cdot t_{3 P}+2 \cdot\left(b_{3 P}\right)^{2} \cdot t_{3 P}+2 \cdot\left(c_{3 P}\right)^{2} \cdot t_{3 P}=0
\end{gathered}
$$

Derivando a equação (6.39) em função de $t_{n P}$, tem-se:

$$
\begin{aligned}
\frac{\partial F}{\partial t_{n P}}=-2 & \cdot a_{n P} \cdot\left(X_{P}\right)-2 \cdot b_{n P} \cdot\left(Y_{P}\right)-2 \cdot c_{n P} \cdot\left(Z_{P}\right)+2 \cdot a_{n P} \cdot\left(X_{C O_{n}}\right)+2 \cdot b_{n P} \cdot\left(Y_{C O_{n}}\right)+ \\
& +2 \cdot c_{n P} \cdot\left(Z_{C O_{n}}\right)+2 \cdot\left(a_{n P}\right)^{2} \cdot t_{n P}+2 \cdot\left(b_{n P}\right)^{2} \cdot t_{n P}+2 \cdot\left(c_{n P}\right)^{2} \cdot t_{n P}
\end{aligned}
$$

Igualando a zero, tem-se:

$$
\begin{gathered}
-2 \cdot a_{n P} \cdot\left(X_{P}\right)-2 \cdot b_{n P} \cdot\left(Y_{P}\right)-2 \cdot c_{n P} \cdot\left(Z_{P}\right)+2 \cdot a_{n P} \cdot\left(X_{C O_{n}}\right)+2 \cdot b_{n P} \cdot\left(Y_{C O_{n}}\right)+ \\
\quad+2 \cdot c_{n P} \cdot\left(Z_{C O_{n}}\right)+2 \cdot\left(a_{n P}\right)^{2} \cdot t_{n P}+2 \cdot\left(b_{n P}\right)^{2} \cdot t_{n P}+2 \cdot\left(c_{n P}\right)^{2} \cdot t_{n P}=0
\end{gathered}
$$

$\mathrm{Na}$ forma matricial, a matriz dos coeficientes das incógnitas será sempre uma matriz quadrada, devido ao fato de as incógnitas serem as coordenadas do alvo $\left(X_{P}, Y_{P}, Z_{P}\right)$ e os parâmetros das retas definidas pelas linhas de visada. Desta maneira, o número de equações é sempre 
igual ao número de estações envolvidas no processo, acrescido de três unidades, ou seja, as coordenadas espaciais do alvo. Assim,

$$
M_{C O E F} \cdot M_{I N C}=M_{I N D}
$$

Sendo,

$M_{C O E F}$ - matriz dos coeficientes das incógnitas

$M_{I N C}$ - matriz das incógnitas

$M_{I N D}$ - matriz dos termos independentes

A solução do problema é dada pela equação (6.58), apresentada a seguir.

$$
M_{I N C}=\left(M_{C O E F}\right)^{-1} \cdot M_{I N D}
$$

Considerando que foram usados três instrumentos, ou seja, três linhas de visadas, a matriz dos coeficientes é estabelecida conforme apresentado a seguir.

$$
M_{C O E F}=\left[\begin{array}{llrrrr}
n & 0 & 0 & -a_{1 P} & -a_{2 P} & -a_{3 P} \\
0 & n & 0 & -b_{1 P} & -b_{2 P} & -b_{3 P} \\
0 & 0 & n & -c_{1 P} & -c_{2 P} & -c_{3 P} \\
-a_{1 P} & -b_{1 P} & -c_{1 P} & 1 & 0 & 0 \\
-a_{2 P} & -b_{2 P} & -c_{2 P} & 0 & 1 & 0 \\
-a_{3 P} & -b_{3 P} & -c_{3 P} & 0 & 0 & 1
\end{array}\right]
$$

Sendo,

$n$ - número de estações. Neste caso, $n=3$

A matriz das incógnitas, neste caso, é uma matriz da ordem $(6 \times 1)$, dada por:

$$
M_{I N C}=\left[\begin{array}{c}
X_{P} \\
Y_{P} \\
Z_{P} \\
t_{1 P} \\
t_{2 P} \\
t_{3 P}
\end{array}\right]
$$

Consequentemente, a matriz dos termos independentes também é da ordem (6x1), dada por: 


$$
M_{I N D}=\left[\begin{array}{c}
\Sigma_{1}^{3}\left(X_{C O_{i=1,2,3}}\right) \\
\Sigma_{1}^{3}\left(Y_{C O_{i=1,2,3}}\right) \\
\Sigma_{1}^{3}\left(Z_{C O_{i=1,2,3}}\right) \\
-\left(a_{1 P} \cdot X_{C O_{1}}+b_{1 P} \cdot Y_{C O_{1}}+c_{1 P} \cdot Z_{C O_{1}}\right) \\
-\left(a_{2 P} \cdot X_{C O_{2}}+b_{2 P} \cdot Y_{C O_{2}}+c_{2 P} \cdot Z_{C O_{2}}\right) \\
-\left(a_{3 P} \cdot X_{C_{3}}+b_{3 P} \cdot Y_{C O_{3}}+c_{3 P} \cdot Z_{C_{3}}\right)
\end{array}\right]
$$

Por meio da solução do sistema de equações (6.58), é possível obter, para o caso particular que envolve três instrumentos de medição, $\left(X_{P}, Y_{P}, Z_{P}, t_{1 P}, t_{2 P}, t_{3 P}\right)$, que são os valores das incógnitas de interesse.

Com os valores das distâncias $t_{i P}$ conhecidas, é possível determinar as coordenadas dos pontos $G_{i}$, a partir das equações (6.31), repetidas abaixo em (6.59).

$$
X G_{i}=X_{C O_{i}}+t_{i P} \cdot a_{i P} \quad Y G_{i}=Y_{C O_{i}}+t_{i P} \cdot b_{i P} \quad Z G_{i}=Z_{C O_{i}}+t_{i P} \cdot c_{i P}
$$

Estas são as coordenadas dos pontos de distância mínima, localizados sobre as retas, definidas pelas linhas de visadas entre os instrumentos e os alvos, em função dos cossenos diretores das mesmas. A precisão das coordenadas dos pontos genéricos $G_{i}$ é definida como o desvio padrão da média destas determinações. Como a média das coordenadas dos pontos genéricos $G_{i}$ é igual ao valor das coordenadas do alvo $P$, os valores obtidos para a precisão das determinações são absolutamente iguais. Assim, a precisão das coordenadas dos pontos $G_{i}$ é dada por,

$$
\sigma_{X G}=\sqrt{\frac{\sum\left(X_{P}-X_{G_{i}}\right)^{2}}{n-1}} \quad \sigma_{Y G}=\sqrt{\frac{\sum\left(Y_{P}-Y_{G_{i}}\right)^{2}}{n-1}} \quad \sigma_{Z G}=\sqrt{\frac{\sum\left(Z_{P}-Z_{G_{i}}\right)^{2}}{n-1}}
$$

Sendo,

$X_{P}, Y_{P}, Z_{P}$ - coordenadas do alvo

$X_{G_{i}}, Y_{G_{i}}, Z_{G_{i}}$ - coordenadas dos pontos sobre as linhas de visada

$n$ - número de estações

A precisão das coordenadas finais de cada alvo $P$ é definida em função da precisão das determinações dos valores das coordenadas dos pontos genéricos $G_{i}$, considerando as $n$ estações envolvidas no processo. Assim, a precisão é calculada em função do desvio padrão da média das coordenadas $\left(X_{G_{i}}, Y_{G_{i}}, Z_{G_{i}}\right)$. Logo,

$$
\sigma_{\overline{X P}}=\frac{\sigma_{X G}}{\sqrt{n}} \quad \sigma_{\overline{Y P}}=\frac{\sigma_{Y G}}{\sqrt{n}} \quad \sigma_{\overline{Z P}}=\frac{\sigma_{Z G}}{\sqrt{n}}
$$

Sendo, 


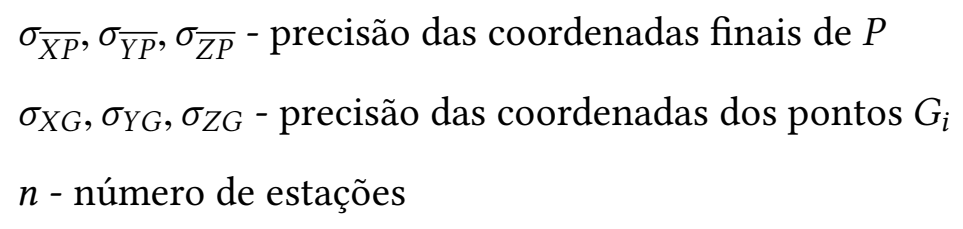

Determinadas as coordenadas do alvo $P$ e suas respectivas precisões, pode-se estimar um valor médio em torno do qual, provavelmente, fique localizado espacialmente o ponto que define a posição do alvo $P$. Neste caso, define-se a precisão do posicionamento espacial do alvo $\sigma_{P}$, como sendo o escalar obtido pela raiz quadrada da soma dos quadrados das precisões das coordenadas espaciais do alvo $P$.

Assim, a precisão espacial do posicionamento do alvo $P$ é calculada por,

$$
\sigma_{P}=\sqrt{\left(\sigma_{\overline{X G}}\right)^{2}+\left(\sigma_{\overline{Y G}}\right)^{2}+\left(\sigma_{\overline{Z G}}\right)^{2}}
$$

Sendo,

$\sigma_{P}$ - precisão do posicionamento espacial do alvo $P$

$\sigma_{\overline{X G}}, \sigma_{\overline{Y G}}, \sigma_{\overline{Z G}}-$ precisão das coordenadas finais de $P$

\subsubsection{Método das distâncias mínimas com ajuste das observações}

Baseando-se no método das distâncias mínimas apresentado acima, foi desenvolvida uma variante, denominado Método das Distâncias Mínimas e Ajuste, que utiliza os mesmos princípios do método anterior, porém, aplicando-se o Método Paramétrico de ajuste de observações para o cálculo das coordenadas do ponto $P$, em que cada estação fixa ou instrumento envolvido no processo fornece três equações de erros. As incógnitas são as coordenadas espaciais do alvo $\left(X_{P}, Y_{P}, Z_{P}\right)$ e as distâncias $d_{i P}^{\prime C}$ das retas $r_{i P}$, definidas pela linha de visada de cada instrumento. São consideradas, então, as equações de erros indicadas nas equações (6.63), (6.64) e (6.65), onde $i=1,2,3, \cdots, n$,

$$
\begin{aligned}
& v_{X_{i}}=X_{P}-\left(X_{i}+a_{i P} \cdot d_{i P}^{\prime C}\right) \\
& v_{Y_{i}}=Y_{P}-\left(Y_{i}+b_{i P} \cdot d_{i P}^{\prime C}\right) \\
& v_{Z_{i}}=Z_{P}-\left(Z_{i}+c_{i P} \cdot d_{i P}^{\prime C}\right)
\end{aligned}
$$

Sendo,

$v_{X_{i}}, v_{Y_{i}}, v_{Z_{i}}$ - distância entre o ponto ajustado e o ponto genérico na interseção da perpendicular à linha de visada

$X_{P}, Y_{P}, Z_{P}$ - coordenadas calculadas para o ponto $P$ 
$X_{i}, Y_{i}, Z_{i}$ - coordenadas conhecidas da estação $I$

$a_{i P}, b_{i P}, c_{i P}$ - cossenos diretores das linhas de visadas entre as estação $I$ e o ponto $P$

$d_{i P}^{\prime C}$ - distância inclinada calculada entre a estação $I$ e o ponto $P$

$\lambda$ - parâmetro da reta de linha de visada $i P$

Para que os resíduos, valores das funções $\left(v_{X_{i}}, v_{Y_{i}}, v_{Z_{i}}\right)$, tornem-se mínimos, para $n$ estações bases, as incógnitas são relacionadas por uma função $F$, definidas por,

$$
F_{m}=F_{m}\left(X_{P}, Y_{P}, Z_{P}, \lambda_{1 P}, \lambda_{2 P}, \lambda_{3 P}\right)
$$

Sendo,

$m=1,2$ e 3

Dessa forma, é possível realizar o ajustamento das observações.

$$
V=A X-L
$$

Sendo,

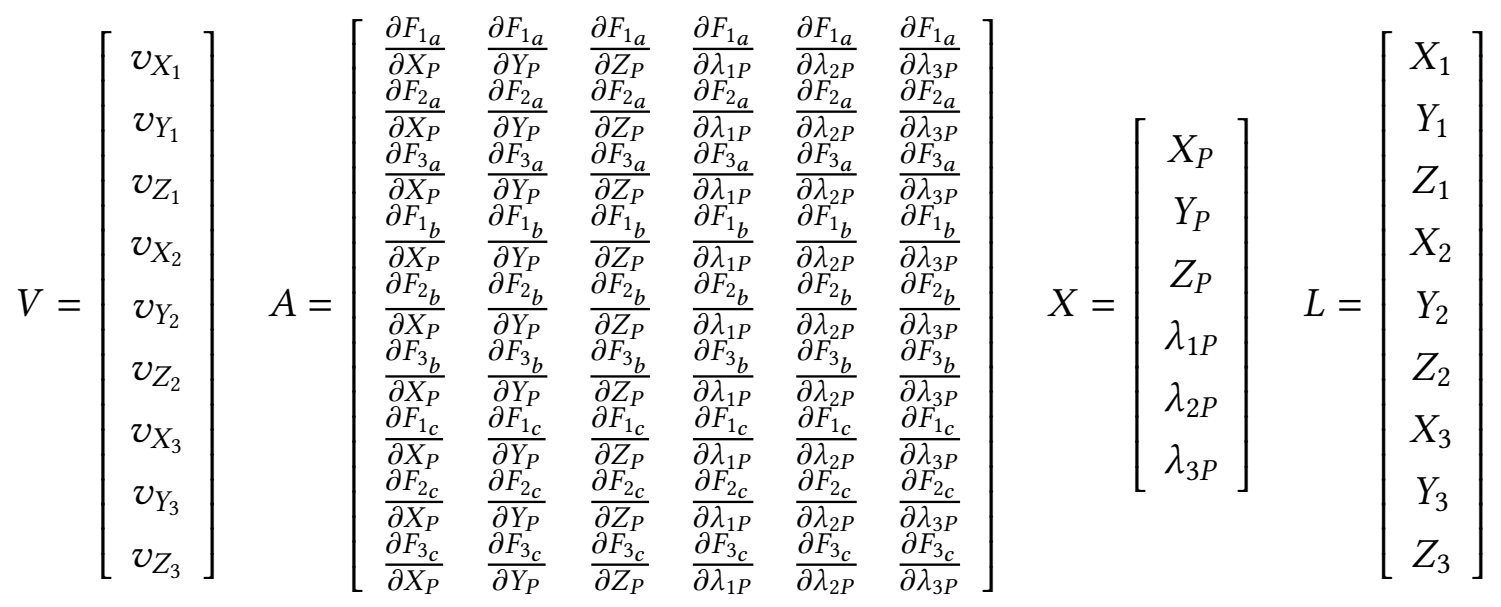

As derivadas parciais que compõem a matriz $A$, são definidas por,

$$
A=\left[\begin{array}{cccccc}
1 & 0 & 0 & -a_{1 P} & 0 & 0 \\
0 & 1 & 0 & -b_{1 P} & 0 & 0 \\
0 & 0 & 1 & -c_{1 P} & 0 & 0 \\
1 & 0 & 0 & 0 & -a_{2 P} & 0 \\
0 & 1 & 0 & 0 & -b_{2 P} & 0 \\
0 & 0 & 1 & 0 & -c_{2 P} & 0 \\
1 & 0 & 0 & 0 & 0 & -a_{3 P} \\
0 & 1 & 0 & 0 & 0 & -b_{3 P} \\
0 & 0 & 1 & 0 & 0 & -c_{3 P}
\end{array}\right]
$$


De acordo com a seção 4.5, tem-se,

$$
X=\left(A^{T} P A\right)^{-1}\left(A^{T} P L\right)
$$

As precisões das incógnitas $X$ podem ser calculadas considerando as equações (4.22) e (4.23).

Dessa forma, é possível determinar as precisões de cada incógnita $X_{P}, Y_{P}$ e $Z_{P}$ e de todos os parâmetros $\lambda_{1 P}, \lambda_{2 P}$ e $\lambda_{3 P}$.

$$
\sigma_{X_{P}}=\sqrt{\hat{\sigma}_{X_{P}}^{2}} \quad \sigma_{Y_{P}}=\sqrt{\hat{\sigma}_{Y_{P}}^{2}} \quad \sigma_{Z_{P}}=\sqrt{\hat{\sigma}_{Z_{P}}^{2}}
$$

Por fim, obtém-se as precisões das coordenadas espaciais $\left(X_{P}, Y_{P}, Z_{P}\right)$.

$$
\sigma_{X_{P} Y_{P} Z_{P}}=\sqrt{\left(\sigma_{X_{P}}\right)^{2}+\left(\sigma_{Y_{P}}\right)^{2}+\left(\sigma_{Z_{P}}\right)^{2}}
$$

\subsubsection{Método de interseção espacial analítica}

Outro método de determinação de coordenadas espaciais sem medição de distância, encontrado na literatura, é o denominado Método de Interseção Espacial Analítica. Ele se baseia, também, na solução de um sistema de equações estabelecidas em função dos cossenos diretores, determinados pelas linhas de visadas de dois ou mais instrumentos topográficos em relação ao alvo. Assim, considerando as relações geométricas da interseção espacial entre duas linhas de visadas, têm-se as equações (6.70) e (6.71), repetidas abaixo para conveniência de leitura. A Figura 6.11 mostra a relação geométrica deste método.

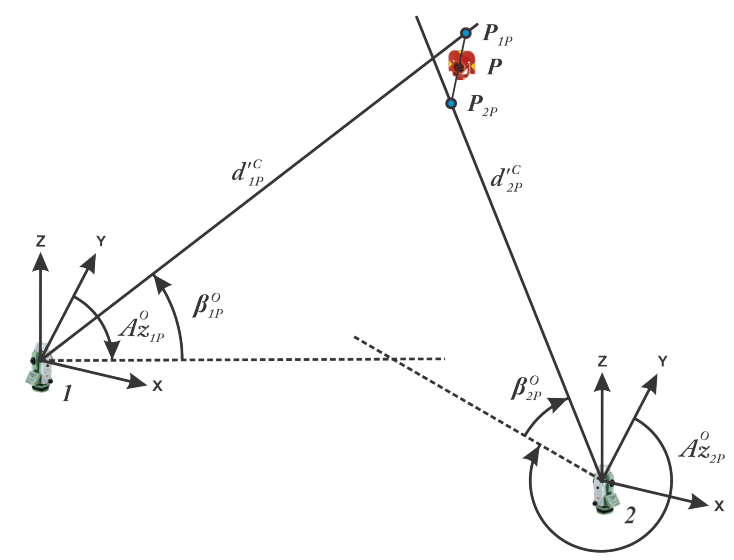

Figura 6.11: Relações geométricas da interseção espacial analítica.

$$
\begin{array}{ll}
a_{1 P}=\cos \left(\beta_{1 P}^{O}\right) \cdot \operatorname{sen}\left(A z_{1 P}^{O}\right) & b_{1 P}=\cos \left(\beta_{1 P}^{O}\right) \cdot \cos \left(A z_{1 P}^{O}\right) \quad c_{1 P}=\operatorname{sen}\left(\beta_{1 P}^{O}\right) \\
a_{2 P}=\cos \left(\beta_{2 P}^{O}\right) \cdot \operatorname{sen}\left(A z_{2 P}^{O}\right) & b_{2 P}=\cos \left(\beta_{2 P}^{O}\right) \cdot \cos \left(A z_{2 P}^{O}\right) \quad c_{2 P}=\operatorname{sen}\left(\beta_{2 P}^{O}\right)
\end{array}
$$


Sendo,

$\beta_{1 P}^{O}$ - ângulo vertical de altura observado no ponto 1 até o alvo $P$

$\beta_{2 P}^{O}$ - ângulo vertical de altura observado no ponto 2 até o alvo $P$

$A z_{1 P}^{O}$ - azimute observado na direção $1 P$

$A z_{2 P}^{O}$ - azimute observado na direção $2 P$

$X_{1}, Y_{1}, Z_{1}$ - coordenadas $3 \mathrm{D}$ do ponto 1

$X_{2}, Y_{2}, Z_{2}$ - coordenadas 3D do ponto 2

$d_{1 P}^{\prime C}$ - distância inclinada calculada entre o ponto 1 e o alvo $P$

$d_{2 P}^{\prime C}$ - distância inclinada calculada entre o ponto 2 e o alvo $P$

Conforme se observa nas equações acima, igualando os termos em $\left(X_{1}, Y_{1}, Z_{1}\right)$ e $\left(X_{2}, Y_{2}, Z_{2}\right)$, têm-se 3 equações e duas incógnitas, que são as distâncias inclinadas calculadas $d_{1 P}^{\prime C}$ e $d_{2 P}^{\prime C}$. A solução para o problema pode ser obtida aplicando-se o método de ajustamento paramétrico. Assim, considerando que a distância entre os dois pontos $P 1$ e $P 2$, determinados pelas visadas que partem das estações 1 e 2, deve ser mínima, pode-se escrever o sistema de equações de erros, conforme indicado nas equações (6.72), (6.73) e (6.74),

$$
\begin{aligned}
& v_{1}=\left(d_{1 P}^{\prime C} \cdot a_{1 P}\right)-\left(d_{2 P}^{\prime C} \cdot a_{2 P}\right)-\left(X_{2}-X_{1}\right) \\
& v_{2}=\left(d_{1 P}^{\prime C} \cdot b_{1 P}\right)-\left(d_{2 P}^{\prime C} \cdot b_{2 P}\right)-\left(Y_{2}-Y_{1}\right) \\
& v_{3}=\left(d_{1 P}^{\prime C} \cdot c_{1 P}\right)-\left(d_{2 P}^{\prime C} \cdot c_{2 P}\right)-\left(Z_{2}-Z_{1}\right)
\end{aligned}
$$

Dessa forma, é possível realizar o ajustamento das observações.

$$
V=A X-L
$$

Sendo,

$$
V=\left[\begin{array}{c}
v_{1} \\
v_{2} \\
v_{3}
\end{array}\right] \quad A=\left[\begin{array}{cc}
a_{1 P} & -a_{2 P} \\
b_{1 P} & -b_{2 P} \\
c_{1 P} & -c_{2 P}
\end{array}\right] \quad X=\left[\begin{array}{c}
d_{1 P}^{\prime C} \\
d_{2 P}^{\prime C}
\end{array}\right] \quad L=\left[\begin{array}{c}
X_{2}-X_{1} \\
Y_{2}-Y_{1} \\
Z_{2}-Z_{1}
\end{array}\right]
$$

De acordo com a seção 4.5 , tem-se,

$$
X=\left(A^{T} P A\right)^{-1}\left(A^{T} P L\right)
$$


Dessa forma, as precisões das incógnitas $X$ podem ser calculadas considerando as equações (4.22) e (4.23). Assim, é possível determinar as precisões de cada incógnita $d_{1 P}^{\prime C}$ e $d_{2 P}^{\prime C}$.

$$
K_{x x}=\hat{\sigma}_{0}^{2} \cdot\left(A^{T} P A\right)^{-1}
$$

Sendo,

$$
\begin{gathered}
K_{x x}=\left[\begin{array}{cc}
\hat{\sigma}_{d_{1 P}^{\prime C}}^{2} & \hat{\sigma}_{12} \\
\hat{\sigma}_{21} & \hat{\sigma}_{d_{2 P}^{\prime C}}^{2}
\end{array}\right] \\
\sigma_{d_{1 P}^{\prime C}}=\sqrt{\hat{\sigma}_{d_{1 P}^{\prime C}}^{2}} \quad \sigma_{d_{2 P}^{\prime C}}=\sqrt{\hat{\sigma}_{d_{2 P}^{\prime}}^{2}}
\end{gathered}
$$

Substituindo os valores de $d_{1 P}^{\prime C}$ e $d_{2 P}^{\prime C}$ nas equações do método polar, obtêm-se os valores das coordenadas dos pontos $P_{1}$ e $P_{2}$. As coordenadas ajustadas do ponto $P$ são calculadas pela média aritmética das coordenadas individuais calculadas em cada linha de visada, conforme as equações (6.79).

$$
\bar{X}_{P}=\frac{X_{1 P}+X_{2 P}}{2} \quad \bar{Y}_{P}=\frac{Y_{1 P}+Y_{2 P}}{2} \quad \bar{Z}_{P}=\frac{Z_{1 P}+Z_{2 P}}{2}
$$

Para os casos em que se utilizam três ou mais instrumentos de medição, ou seja $n>2$, podem-se realizar os cálculos, considerando combinações aleatórias, tomadas duas a duas. E, por meio dessas combinações, extrair a média aritmética $\left(\bar{X}_{1,2,3, \cdots, n, P}, \bar{Y}_{1,2,3, \cdots, n, P}, \bar{Z}_{1,2,3, \cdots, n, P}\right)$, para o terno de coordenadas do ponto $P$.

\subsection{Métodos multipolares com medições de distâncias}

Quando o ponto $P$ a ser determinado é um ponto acessível, ou seja, localizado de forma que se possa colocar um prisma refletor sobre ele, para a medição de distâncias, o método de determinação de coordenadas comumente usado é o polar. Este método, contudo, não produz a melhor solução para os casos em que o ponto $P$ é visado a partir de várias estações ao mesmo tempo, conforme apresentado nas seções anteriores. Para estes casos, as soluções indicadas baseiamse no estabelecimento de equações de erros em função das observações e das incógnitas e, consequentemente, realiza-se o ajustamento pelo MMQ.

\subsubsection{Métodos de minimização dos ângulos e das distâncias medidas}

Um dos métodos de determinação de coordenadas espaciais, com excesso de visadas testado, é o Método da Minimização dos ângulos e das distâncias medidas. Considera-se, neste caso, que as melhores coordenadas para o ponto $P$ são obtidas a partir da minimização dos erros residuais dos azimutes, dos ângulos verticais e das distâncias medidas, conforme apresentado na sequência. 
6.3.2 Método da minimização dos deslocamentos em função dos azimutes, dos ângulos verticais e das distâncias inclinadas (relação geométrica)

O desenvolvimento matemático do modelo baseia-se na determinação dos erros de posicionamento cometidos nas medições dos azimutes $A z_{i P}^{O}$, nas medições dos ângulos verticais de altura $\beta_{i P}^{O}$ e nas medições das distâncias inclinadas $d_{i P}^{\prime O}$, efetuadas em cada linha de visada, em relação ao ponto $P$. Assim, para os azimutes medidos, tem-se a condição geométrica indicada na Figura 6.12.

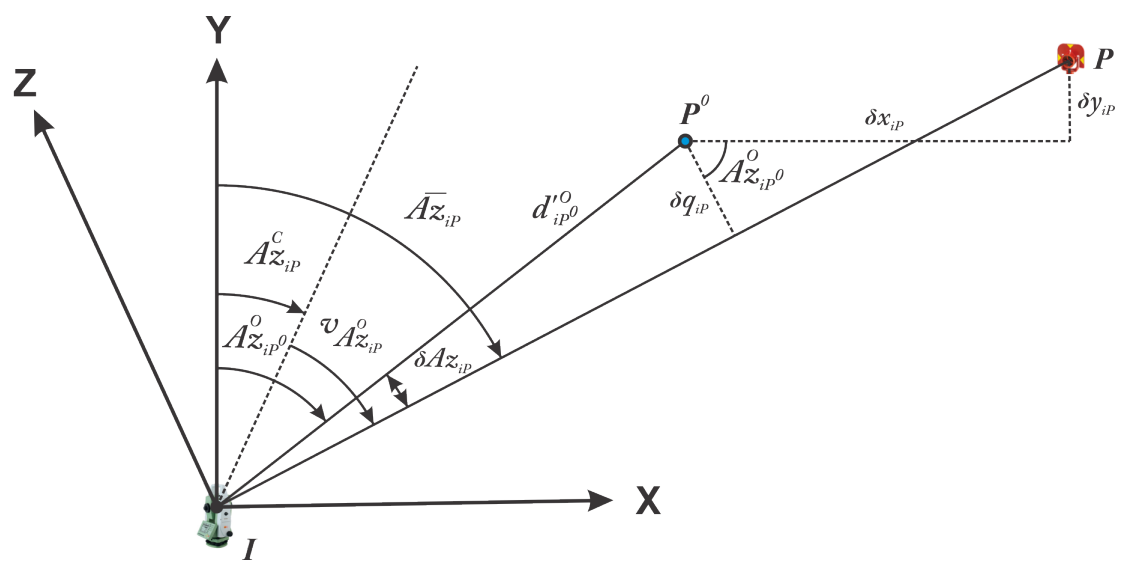

Figura 6.12: Relação geométrica para azimute.

Sendo,

$P^{0}$ - ponto provisório

$A z_{i P^{0}}^{O}$ - azimute observado da direção $i P^{0}$

$A z_{i P}^{C}$ - azimute calculado da direção $i P$

$d_{i P^{0}}^{\prime O}$ - distância inclinada observada entre $i P^{0}$

$d_{i P}^{\prime C}$ - distância inclinada calculada entre $i P$

$\delta A z_{i P}$ - valor do acréscimo para obtenção do ângulo ajustado

$\delta q, \delta x, \delta y$ - valor dos acréscimos para obtenção das coordenadas ajustadas

$\bar{A} z_{i P}$ - azimute ajustado da direção $i P$

Tem-se que,

$$
\begin{gathered}
\delta q=d_{i P^{0}}^{\prime O} \cdot \delta A z \\
\delta q=\delta x \cdot \cos A z_{i P^{0}}^{O}-\delta y \cdot \operatorname{sen} A z_{i p^{0}}^{O}-\delta z \cdot 0 \\
d_{i P^{0}}^{O O} \cdot \delta A z=\delta x \cdot \cos A z_{i P^{0}}^{O}-\delta y \cdot \operatorname{sen} A z_{i P^{0}}^{O}-\delta z \cdot 0
\end{gathered}
$$




$$
\delta A z=\frac{\cos A z_{i P^{0}}^{O}}{d_{i P^{0}}^{\prime O}} \cdot \delta x-\frac{\operatorname{sen} A z_{i P^{0}}^{O}}{d_{i P^{0}}^{\prime O}} \cdot \delta y-0 \cdot \delta z
$$

Considerando,

$$
k_{i P}=\frac{\cos A z_{i P^{0}}^{O}}{d_{i P^{0}}^{\prime O}} \quad m_{i P}=-\frac{\operatorname{sen} A z_{i P^{0}}^{O}}{d_{i P^{0}}^{\prime O}} \quad n_{i P}=0
$$

Obtém-se,

$$
\delta A z=k_{i P} \cdot \delta x+m_{i P} \cdot \delta y+n_{i P} \cdot \delta z
$$

Da mesma forma,

$$
\begin{aligned}
& \overline{A z}=A z_{i P^{0}}^{O}+\delta A z \\
& \overline{A z}=A z_{i P}^{C}+v_{A z_{i P}^{O}}
\end{aligned}
$$

Igualando os termos acima, tem-se:

$$
\begin{aligned}
& A z_{i P^{0}}^{O}+\delta A z=A z_{i P}^{C}+v_{A z_{i P}^{O}} \\
& v_{A z_{i P}^{O}}=\delta A z+A z_{i P^{0}}^{O}-A z_{i P}^{C}
\end{aligned}
$$

Obtém-se a equação de erros para o azimute, conforme indicado a seguir,

$$
v_{A z_{i P}^{O}}=k_{i P} \cdot \delta x+m_{i P} \cdot \delta y+n_{i P} \cdot \delta z-\left(A z_{i P^{0}}^{O}-A z_{i P}^{C}\right)
$$

Para os ângulos verticais de altura medidos, tem-se a condição geométrica indicada na Figura 6.13. 


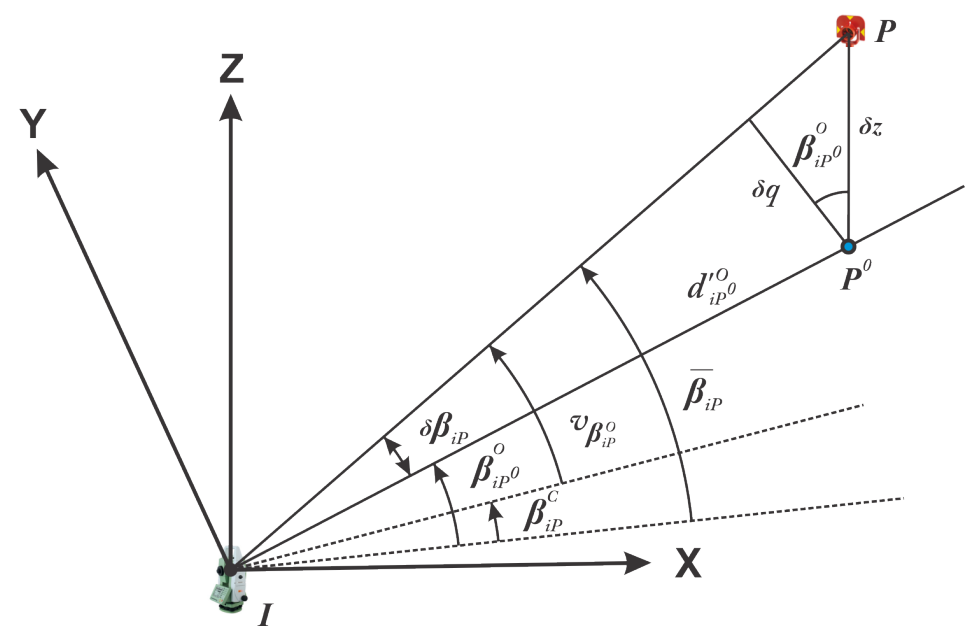

Figura 6.13: Relação geométrica ângulo vertical de altura.

Sendo,

$P^{0}$ - ponto provisório

$A z_{i P}^{O}$ - azimute medido da direção $i P$

$A z_{i P}^{C}$ - azimute calculado da direção $i P$

$\beta_{i P}^{O}$ - ângulo vertical de altura medido entre $i P$

$\beta_{i P}^{C}$ - ângulo vertical de altura calculado entre $i P$

$d_{i P}^{\prime O}$ - distância inclinada medida entre $i P$

$d_{i P}^{\prime C}$ - distância inclinada calculada entre $i P$

$\delta X_{P}, \delta Y_{P}, \delta Z_{P}$ - valor dos acréscimos para obtenção das coordenadas ajustadas

$$
\begin{gathered}
\delta q=\cos \beta_{i P^{0}}^{O} \cdot \delta z \\
\delta q=d_{i P^{0}}^{O} \cdot \delta \beta \\
\delta \beta=\frac{\cos \beta_{i P^{0}}^{O}}{d_{i P^{0}}^{\prime O}} \cdot \delta z
\end{gathered}
$$

Considerando,

$$
h_{i P}=0 \quad g_{i P}=0 \quad l_{i P}=\frac{\cos \beta_{i P^{0}}^{O}}{d_{i P^{0}}^{\prime O}}
$$


Obtém-se,

$$
\delta \beta=h_{i P} \cdot \delta x+g_{i P} \cdot \delta y+l_{i P} \cdot \delta z
$$

Da mesma forma,

$$
\begin{aligned}
& \bar{\beta}=\beta_{i P^{0}}^{O}+\delta \beta \\
& \bar{\beta}=\beta_{i P}^{C}+v_{\beta_{i P}^{O}}
\end{aligned}
$$

Igualando os termos acima, tem-se:

$$
\begin{array}{r}
\bar{\beta}=\beta_{i P^{0}}^{O}+\delta \beta=\beta^{C}+v_{\beta_{i P}^{O}} \\
v_{\beta_{i P}^{O}}=\delta \beta+\beta_{i P^{0}}^{O}-\beta_{i P}^{C}
\end{array}
$$

Obtém-se a equação de erros para o ângulo vertical, conforme indicado a seguir;

$$
v_{\beta_{i P}^{O}}=h_{i P} \cdot \delta x+g_{i P} \cdot \delta y+l_{i P} \cdot \delta z-\left(\beta_{i P^{0}}^{O}-\beta_{i P}^{C}\right)
$$

Para a distância, deve-se considerar a equação da distância inclinada indicada a seguir,

$$
d_{i P}^{\prime C}=\sqrt{\left[\left(X_{P}-X_{i}\right) \operatorname{sen}\left(A z_{i P}^{C}\right)+\left(Y_{P}-Y_{i}\right) \cos \left(A z_{i P}^{C}\right)\right]^{2}+\left(Z_{P}-Z_{i}\right)^{2}}
$$

A equação de erros gerada pela equação acima é dada por,

$$
v_{d_{i P}^{\prime O}}=\frac{\partial d_{i P}^{\prime C}}{\partial X_{P}} \delta X_{P}+\frac{\partial d_{i P}^{\prime C}}{\partial Y_{P}} \delta Y_{P}+\frac{\partial d_{i P}^{\prime C}}{\partial Z_{P}} \delta Z_{P}-\left(d_{i P}^{\prime O}-d_{i P}^{\prime C}\right)
$$

Sendo,

$$
\begin{gathered}
\frac{\partial d_{i P}^{\prime C}}{\partial X_{P}}=\frac{\left[\left(X_{P}-X_{i}\right) \cdot \operatorname{sen}\left(A z_{i P}^{C}\right)+\left(Y_{P}-Y_{i}\right) \cdot \cos \left(A z_{i P}^{C}\right)\right] \cdot \operatorname{sen}\left(A z_{i P}^{C}\right)}{d_{i P}^{\prime C}} \\
\frac{\partial d_{i P}^{\prime C}}{\partial Y_{P}}=\frac{\left[\left(X_{P}-X_{i}\right) \cdot \operatorname{sen}\left(A z_{i P}^{C}\right)+\left(Y_{P}-Y_{i}\right) \cdot \cos \left(A z_{i P}^{C}\right)\right] \cdot \cos \left(A z_{i P}^{C}\right)}{d_{i P}^{\prime C}} \\
\frac{\partial d_{i P}^{\prime C}}{\partial Z_{P}}=\frac{\left(Z_{P}-Z_{i}\right)}{d_{i P}^{\prime C}}
\end{gathered}
$$

Sendo, 
$A z_{i P}^{O}$ - azimute medido da direção $i P$

$A z_{i P}^{C}$ - azimute calculado da direção $i P$

$\beta_{i P}^{O}$ - ângulo vertical de altura medido entre $i P$

$\beta_{i P}^{C}$ - ângulo vertical de altura calculado entre $i P$

$d_{i P}^{\prime O}$ - distância inclinada medida entre $i P$

$d_{i P}^{\prime C}$ - distância inclinada calculada entre $i P$

$\delta X_{P}, \delta Y_{P}, \delta Z_{P}$ - valor dos acréscimos para obtenção das coordenadas ajustadas

Agrupando as equações de erros, tem-se,

$$
\begin{gathered}
v_{A z_{i P}^{O}}=k_{i P} \cdot \delta x+m_{i P} \cdot \delta y+n_{i P} \cdot \delta z-\left(A z_{i P}^{O}-A z_{i P}^{C}\right) \\
v_{\beta_{i P}^{O}}=h_{i P} \cdot \delta x+g_{i P} \cdot \delta y+l_{i P} \cdot \delta z-\left(\beta_{i P}^{O}-\beta_{i P}^{C}\right) \\
v_{d_{i P}^{\prime}}=\frac{\partial d_{i P}^{\prime C}}{\partial X_{P}} \delta X_{P}+\frac{\partial d_{i P}^{\prime C}}{\partial Y_{P}} \delta Y_{P}+\frac{\partial d_{i P}^{\prime C}}{\partial Z_{P}} \delta Z_{P}-\left(d_{i P}^{\prime O}-d_{i P}^{\prime C}\right)
\end{gathered}
$$

Nestas condições, considerando o caso de três visadas partindo das estações 1, 2 e 3, tem-se,

$$
V=A X-L
$$

Sendo,

$$
V=\left[\begin{array}{c}
v_{A z_{1 P}^{O}} \\
v_{\beta_{1 P}^{O}} \\
v_{d_{1 P}^{\prime O}} \\
v_{A z_{2 P}^{O}} \\
v_{\beta_{2 P}^{O}} \\
v_{d_{2 P}^{\prime O}} \\
v_{A z_{3 P}^{O}} \\
v_{\beta_{3 P}^{O}} \\
v_{d_{3 P}^{\prime O}}
\end{array}\right] \quad A=\left[\begin{array}{ccc}
k_{1 P} & m_{1 P} & n_{1 P} \\
h_{1 P} & g_{1 P} & l_{1 P} \\
\frac{\partial d_{1 P}^{\prime C}}{\partial X_{P}} & \frac{\partial d_{1 P}^{\prime C}}{\partial Y_{P}} & \frac{\partial d_{1 P}^{\prime C}}{\partial Z_{P}} \\
k_{2 P} & m_{2 P} & n_{2 P} \\
h_{2 P} & g_{2 P} & l_{2 P} \\
\frac{\partial d_{2 P}^{\prime C}}{\partial X_{P}} & \frac{\partial d_{2 P}^{\prime C}}{\partial Y_{P}} & \frac{\partial d_{2 P}^{\prime C}}{\partial Z_{P}} \\
k_{3 P} & m_{3 P} & n_{3 P} \\
h_{3 P} & g_{3 P} & l_{3 P} \\
\frac{\partial d_{3 P}^{\prime}}{\partial X_{P}} & \frac{\partial d_{3 P}^{\prime C}}{\partial Y_{P}} & \frac{\partial d_{3 P}^{\prime C}}{\partial Z_{P}}
\end{array}\right] \quad X=\left[\begin{array}{c}
\delta X_{P} \\
\delta Y_{P} \\
\delta Z_{P}
\end{array}\right] \quad L=\left[\begin{array}{c}
A z_{1 P}^{O}-A z_{1 P}^{C} \\
\beta_{1 P}^{O}-\beta_{1 P}^{C} \\
d_{1 P}^{\prime O}-d_{1 P}^{\prime C} \\
A z_{2 P}^{O}-A z_{2 P}^{C} \\
\beta_{2 P}^{O}-\beta_{2 P}^{C} \\
d_{2 P}^{\prime O}-d_{2 P}^{\prime C} \\
A z_{3 P}^{O}-A z_{3 P}^{C} \\
\beta_{3 P}^{O}-\beta_{3 P}^{C} \\
d_{3 P}^{O}-d_{3 P}^{C}
\end{array}\right]
$$

De acordo com a seção 4.5 , tem-se, 


$$
X=\left(A^{T} P A\right)^{-1}\left(A^{T} P L\right)
$$

As precisões das incógnitas $X$ podem ser calculadas considerando as equações (4.22) e (4.23). Dessa forma, é possível determinar as precisões de cada incógnita $X_{P}, Y_{P}$ e $Z_{P}$.

$$
\sigma_{X_{P}}=\sqrt{\hat{\sigma}_{X_{P}}^{2}} \quad \sigma_{Y_{P}}=\sqrt{\hat{\sigma}_{Y_{P}}^{2}} \quad \sigma_{Z_{P}}=\sqrt{\hat{\sigma}_{Z_{P}}^{2}}
$$

E por fim, as precisões das coordenadas espaciais $\left(X_{P}, Y_{P}, Z_{P}\right)$.

$$
\sigma_{X_{P} Y_{P} Z_{P}}=\sqrt{\left(\sigma_{X_{P}}\right)^{2}+\left(\sigma_{Y_{P}}\right)^{2}+\left(\sigma_{Z_{P}}\right)^{2}}
$$

\subsubsection{Método da minimização dos resíduos das funções trigonométricas em função dos} azimutes, dos ângulos verticais e das distâncias inclinadas (relação analítica)

O desenvolvimento matemático do modelo baseia-se na determinação dos erros de posicionamento cometidos nas medições dos azimutes $A z_{i P}^{O}$, nas medições dos ângulos verticais de altura $\beta_{i P}^{O}$ e nas medições das distâncias inclinadas $d_{i P}^{\prime O}$, efetuadas em cada visada relativa ao ponto $P$. Assim, para os azimutes medidos, tem-se a condição analítica.

$$
\begin{gathered}
A z_{i P}^{C}=\operatorname{arctg}\left(\frac{X_{P}-X_{i}}{Y_{P}-Y_{i}}\right) \\
\beta_{i P}^{C}=\operatorname{arctg}\left(\frac{Z_{P}-Z_{i}}{\sqrt{\left(X_{P}-X_{i}\right)^{2}+\left(Y_{P}-Y_{i}\right)^{2}}}\right) \\
d_{i P}^{\prime C}=\sqrt{\left[\left(X_{P}-X_{i}\right) \operatorname{sen}\left(A z_{i P}^{C}\right)+\left(Y_{P}-Y_{i}\right) \cos \left(A z_{i P}^{C}\right)\right]^{2}+\left(Z_{P}-Z_{i}\right)^{2}}
\end{gathered}
$$

As equações de erros, geradas pelos modelos acima, são iguais a,

$$
\begin{gathered}
v_{A z_{i P}^{O}}=\frac{\partial A z_{i P}^{C}}{\partial X_{P}} \delta X_{P}+\frac{\partial A z_{i P}^{C}}{\partial Y_{P}} \delta Y_{P}+\frac{\partial A z_{i P}^{C}}{\partial Z_{P}} \delta Z_{P}-\left(A z_{i P}^{O}-A z_{i P}^{C}\right) \\
v_{\beta_{i P}^{O}}=\frac{\partial \beta_{i P}^{C}}{\partial X_{P}} \delta X_{P}+\frac{\partial \beta_{i P}^{C}}{\partial Y_{P}} \delta Y_{P}+\frac{\partial \beta_{i P}^{C}}{\partial Z_{P}} \delta Z_{P}-\left(\beta_{i P}^{O}-\beta_{i P}^{C}\right) \\
v_{d_{i P}^{\prime O}}=\frac{\partial d_{i P}^{\prime C}}{\partial X_{P}} \delta X_{P}+\frac{\partial d_{i P}^{\prime C}}{\partial Y_{P}} \delta Y_{P}+\frac{\partial d_{i P}^{\prime C}}{\partial Z_{P}} \delta Z_{P}-\left(d_{i P}^{\prime O}-d_{i P}^{\prime C}\right)
\end{gathered}
$$

Sendo,

$$
\frac{\partial A z_{i P}^{C}}{\partial X_{P}}=\frac{\left(Y_{P}-Y_{i}\right)}{d_{i P}^{\prime C}}
$$




$$
\begin{gathered}
\frac{\partial A z_{i P}^{C}}{\partial Y_{P}}=-\frac{\left(X_{P}-X_{i}\right)}{d_{i P}^{\prime C}} \\
\frac{\partial A z_{i P}^{C}}{\partial Z_{P}}=0 \\
\frac{\partial \beta_{i P}^{C}}{\partial X_{P}}=-\frac{\left(Z_{P}-Z_{i}\right)\left(X_{P}-X_{i}\right)}{\sqrt{\left(X_{P}-X_{i}\right)^{2}+\left(Y_{P}-Y_{i}\right)^{2}} \cdot d_{i P}^{\prime C}} \\
\frac{\partial \beta_{i P}^{C}}{\partial Y_{P}}=-\frac{\left(Z_{P}-Z_{i}\right)\left(Y_{P}-Y_{i}\right)}{\sqrt{\left(X_{P}-X_{i}\right)^{2}+\left(Y_{P}-Y_{i}\right)^{2}} \cdot d_{i P}^{\prime C}} \\
\frac{\partial \beta_{i P}^{C}}{\partial Z_{P}}=\frac{\sqrt{\left(X_{P}-X_{i}\right)^{2}+\left(Y_{P}-Y_{i}\right)^{2}}}{d_{i P}^{\prime C}} \\
\frac{\partial d_{i P}^{\prime C}}{\partial Y_{P}}=\frac{\left[\left(X_{P}-X_{i}\right) \cdot \operatorname{sen}\left(A z_{i P}^{C}\right)+\left(Y_{P}-Y_{i}\right) \cdot \cos \left(A z_{i P}^{C}\right)\right] \cdot \operatorname{sen}\left(A z_{i P}^{C}\right)}{d_{i P}^{\prime C}} \\
\frac{\partial d_{i P}^{\prime C}}{\partial Z_{P}}=\frac{\left(Z_{P}-Z_{i}\right)}{d_{i P}^{\prime C}} \\
d_{i P}^{\prime C}
\end{gathered}
$$

Sendo,

$A z_{i P}^{O}$ - azimute medido da direção $i P$

$A z_{i P}^{C}$ - azimute calculado da direção $i P$

$\beta_{i P}^{O}$ - ângulo vertical de altura medido entre $i P$

$\beta_{i P}^{C}$ - ângulo vertical de altura calculado entre $i P$

$d_{i P}^{\prime O}$ - distância inclinada medida entre $i P$

$d_{i P}^{\prime C}$ - distância inclinada calculada entre $i P$

$\delta X_{P}, \delta Y_{P}, \delta Z_{P}$ - valor dos acréscimos para obtenção das coordenadas ajustadas

Nessas condições, considerando o caso de três visadas partindo das estações 1, 2 e 3, tem-se,

$$
V=A X-L
$$

Sendo, 


$$
V=\left[\begin{array}{c}
v_{A z_{1 P}^{O}} \\
v_{\beta_{1 P}^{O}} \\
v_{d_{1 P}^{\prime O}} \\
v_{A z_{2 P}^{O}} \\
v_{\beta_{2 P}^{O}} \\
v_{d_{2 P}^{\prime O}} \\
v_{A z_{3 P}^{O}} \\
v_{\beta_{3 P}} \\
v_{d_{3 P}^{\prime O}}
\end{array}\right] \quad A=\left[\begin{array}{lll}
\frac{\partial A z_{1 P}^{C}}{\partial X_{P}} & \frac{\partial A z_{1 P}^{C}}{\partial Y_{P}} & \frac{\partial A z_{1 P}^{C}}{\partial Z_{P}} \\
\frac{\partial \beta_{1 P}^{C}}{\partial X_{P}} & \frac{\partial \beta_{1 P}^{C}}{\partial Y_{P}} & \frac{\partial \beta_{1 P}^{C}}{\partial Z_{P}} \\
\frac{\partial d_{1 P}^{\prime C}}{\partial X_{P}} & \frac{\partial d_{1 P}^{\prime C}}{\partial Y_{P}} & \frac{\partial d_{1 P}^{\prime}}{\partial Z_{P}} \\
\frac{\partial A z_{2 P}^{C}}{\partial X_{P}} & \frac{\partial A z_{2 P}^{C}}{\partial Y_{P}} & \frac{\partial A z_{2 P}^{C}}{\partial Z_{P}} \\
\frac{\partial \beta_{2 P}^{C}}{\partial X_{P}} & \frac{\partial \beta_{2 P}^{C}}{\partial Y_{P}} & \frac{\partial \beta_{2 P}^{C}}{\partial Z_{P}} \\
\frac{\partial d_{2 P}^{\prime}}{\partial X_{P}} & \frac{\partial d_{2 P}^{\prime C}}{\partial Y_{P}} & \frac{\partial d_{2 P}^{\prime C}}{\partial Z_{P}} \\
\frac{\partial A z_{3 P}^{C}}{\partial X_{P}} & \frac{\partial A z_{3 P}^{C}}{\partial Y_{P}} & \frac{\partial A z_{3 P}^{C}}{\partial Z_{P}} \\
\frac{\partial \beta_{3 P}^{C}}{\partial X_{P}} & \frac{\partial \beta_{3 P}^{C}}{\partial Y_{P}} & \frac{\partial \beta_{3 P}^{C}}{\partial Z_{P}} \\
\frac{\partial d_{3 P}^{\prime}}{\partial X_{P}} & \frac{\partial d_{3 P}^{\prime C}}{\partial Y_{P}} & \frac{\partial d_{3 P}^{\prime}}{\partial Z_{P}}
\end{array}\right] \quad X=\left[\begin{array}{c}
\delta X_{P} \\
\delta Y_{P} \\
\delta Z_{P}
\end{array}\right] \quad L=\left[\begin{array}{c}
A z_{1 P}^{O}-A z_{1 P}^{C} \\
\beta_{1 P}^{O}-\beta_{1 P}^{C} \\
d_{1 P}^{\prime O}-d_{1 P}^{\prime C} \\
A z_{2 P}^{O}-A z_{2 P}^{C} \\
\beta_{2 P}^{O}-\beta_{2 P}^{C} \\
d_{2 P}^{\prime O}-d_{2 P}^{\prime C} \\
A z_{3 P}^{O}-A z_{3 P}^{C} \\
\beta_{3 P}^{O}-\beta_{3 P}^{C} \\
d_{3 P}^{\prime O}-d_{3 P}^{\prime C}
\end{array}\right]
$$

De acordo com a seção 4.5, tem-se,

$$
X=\left(A^{T} P A\right)^{-1}\left(A^{T} P L\right)
$$

As precisões das incógnitas $X$ podem ser calculadas considerando as equações (4.22) e (4.23).

Dessa forma, é possível determinar as precisões de cada incógnita $X_{P}, Y_{P}$ e $Z_{P}$.

$$
\sigma_{X_{P}}=\sqrt{\hat{\sigma}_{X_{P}}^{2}} \quad \sigma_{Y_{P}}=\sqrt{\hat{\sigma}_{Y_{P}}^{2}} \quad \sigma_{Z_{P}}=\sqrt{\hat{\sigma}_{Z_{P}}^{2}}
$$

E por fim, as precisões das coordenadas espaciais $\left(X_{P}, Y_{P}, Z_{P}\right)$.

$$
\sigma_{X_{P} Y_{P} Z_{P}}=\sqrt{\left(\sigma_{X_{P}}\right)^{2}+\left(\sigma_{Y_{P}}\right)^{2}+\left(\sigma_{Z_{P}}\right)^{2}}
$$




\section{Capítulo}

\section{MÉTODO ITERATIVO PARA DETERMINAÇÃO DE COORDENADAS ESPACIAIS}

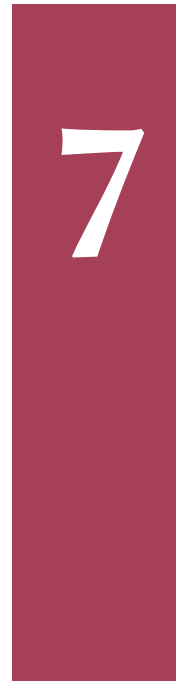

Para o processo de determinação de coordenadas espaciais do alvo com deslocamento controlado pelo braço robótico, foi desenvolvido e aplicado nesta tese, dois modelos matemáticos, que se utilizam de técnicas de convergência.

Dessa forma, o objetivo principal deste capítulo é obter soluções matemáticas para determinar coordenadas espaciais, utilizando-se três estações totais robóticas de duas formas distintas. Primeiramente, consideraremos o caso de três estações totais robóticas, que podem ser combinadas de duas a duas e, por fim, trata-se o caso de três estações totais robóticas, interagindo de forma direta. Para tanto, utilizou-se o Método de Newton Generalizado, para gerar uma sequência de aproximações sucessivas que converge para as coordenadas espaciais, o mais próximo possível do valor real do alvo. Para o desenvolvimento do modelo a ser apresentado, tem-se a necessidade de resolver sistemas de equações não lineares e, maiores detalhes sobre o Método de Newton Generalizado, podem ser vistos em Conte e De-Boor (1980), Polyak (2007) e Burden, Faires e Burden (2016). Estes métodos admitem que as linhas de visada são concorrentes no alvo e, ainda, que os erros nas medições em cada geometria estão implícitos nos vértices, isto é, na interseção das linhas de visada. Os erros acima referidos englobam condições atmosféricas, defeitos sistemáticos dos instrumentos de medição e todos os outros fatores que podem afetar as medições. 


\subsection{Método iterativo de aproximações sucessivas, para determinação de coordenadas espaciais, considerando duas estações totais ro- bóticas}

Nesta seção será apresentada a modelagem matemática proposta, desenvolvida no Laboratório de Geomática do STT, com a finalidade de obter coordenadas espaciais para monitoramento estrutural. A ideia inicial é estabelecer uma relação entre os parâmetros de direções e de distância inclinada. Por meio da Figura 7.1, desenvolveram-se as equações (7.1), (7.2) e (7.3).

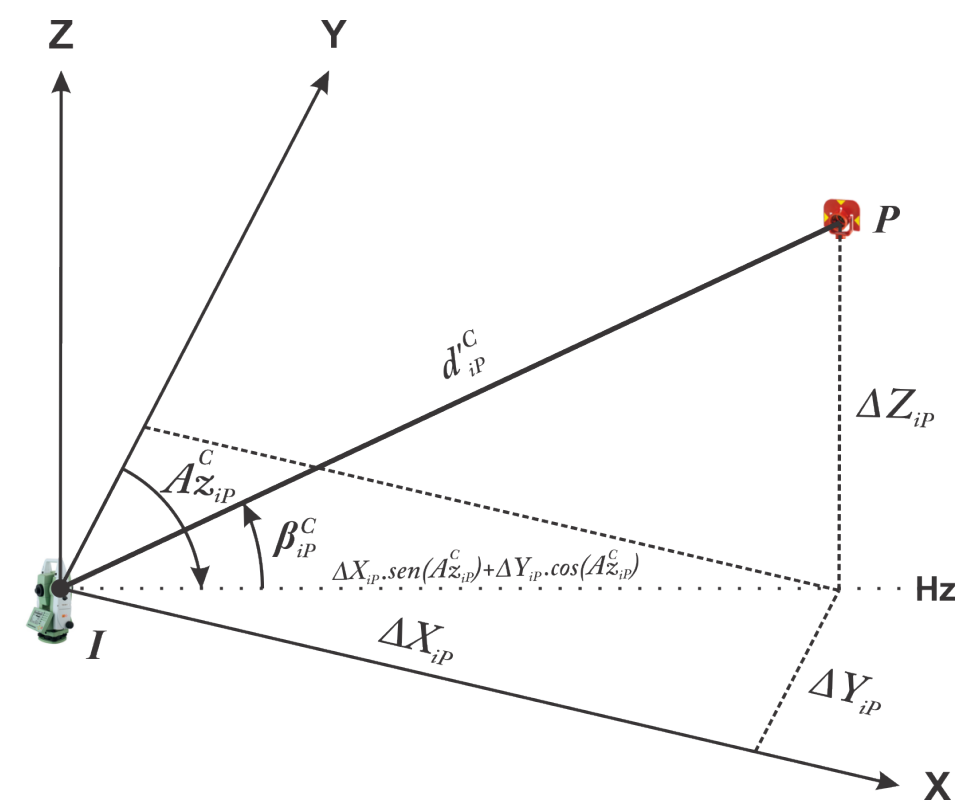

Figura 7.1: Relações geométricas para obtenção das equações das distâncias.

$$
\begin{gathered}
A z_{i P}^{C}=\operatorname{arctg}\left(\frac{X_{P}-X_{i}}{Y_{P}-Y_{i}}\right) \\
\beta_{i P}^{C}=\operatorname{arctg}\left(\frac{Z_{P}-Z_{i}}{\sqrt{\left(X_{P}-X_{i}\right)^{2}+\left(Y_{P}-Y_{i}\right)^{2}}}\right) \\
d_{i p}^{\prime C}=\sqrt{\left[\left(X_{P}-X_{i}\right) \operatorname{sen}\left(A z_{i P}^{C}\right)+\left(Y_{P}-Y_{i}\right) \cos \left(A z_{i P}^{C}\right)\right]^{2}+\left(Z_{P}-Z_{i}\right)^{2}}
\end{gathered}
$$

Da equação (7.2), obtém-se a seguinte relação,

$$
\Delta Z_{i P}=\sqrt{\left(\Delta X_{i P}\right)^{2}+\left(\Delta Y_{i P}\right)^{2}} \cdot \operatorname{tg}\left(\beta_{i P}^{C}\right)
$$

Sendo,

$\Delta X_{i P}=X_{P}-X_{i}$

$\Delta Y_{i P}=Y_{P}-Y_{i}$ 
7.1. Método iterativo de aproximações sucessivas, para determinação de coordenadas espaciais, considerando duas estações totais robóticas

$i=1 \mathrm{e} 2$

$A z_{i P}^{O}$ - azimute medido da direção $i P$

$A z_{i P}^{C}$ - azimute da direção $i P$, calculado pela equação (7.1)

$\beta_{i P}^{O}$ - ângulo vertical de altura da direção $i P$, medido pelo sensor

$\beta_{i P}^{C}$ - ângulo vertical de altura da direção $i P$, calculado pela equação (7.2)

$d_{i p}^{\prime O}$ - distância inclinada medida entre $i P$ pelo sensor

$d_{i p}^{\prime C}$ - distância inclinada calculada entre $i P$, pela equação (7.3)

$X_{i}, Y_{i}, Z_{i}$ - coordenadas conhecidas do ponto $I$ (estação base)

$X_{P}, Y_{P}, Z_{P}$ - coordenadas espaciais a serem calculadas para o ponto $P$ (alvo)

$X_{P}^{0}, Y_{P}^{0}, Z_{P}^{0}$ - coordenadas aproximadas para o ponto $P$ (chute inicial para o alvo)

$\delta X_{P}, \delta Y_{P}, \delta Z_{P}$ - valor dos acréscimos para obtenção das coordenadas espaciais ajustadas

Devido ao fato de se usar duas estações totais robóticas por vez, dentre as três, é preciso substituir a equação (7.4) em (7.3) e considerar os parâmetros medidos $A z_{i P}^{O}$, $\beta_{i P}^{O}$ e $d_{i P}^{\prime O}$ na equação (7.3), de tal forma que gere um sistema não linear. De fato, segue da equação (7.5) o desenvolvimento matemático do modelo.

$$
\begin{gathered}
d_{i P}^{\prime O}=\sqrt{\left[\left(X_{P}-X_{i}\right) \operatorname{sen}\left(A z_{i P}^{O}\right)+\left(Y_{P}-Y_{i}\right) \cos \left(A z_{i P}^{O}\right)\right]^{2}+\left[\left(\Delta X_{i P}\right)^{2}+\left(\Delta Y_{i P}\right)^{2}\right] \cdot \operatorname{tg}^{2}\left(\beta_{i P}^{O}\right)} \\
\left(d_{i P}^{\prime O}\right)^{2}=\left(\Delta X_{i P}\right)^{2} \cdot \operatorname{sen}^{2}\left(A z_{i P}^{O}\right)+2 \cdot \Delta X_{i P} \cdot \Delta Y_{i P} \cdot \operatorname{sen}\left(A z_{i P}^{O}\right) \cdot \cos \left(A z_{i P}^{O}\right)+ \\
+\left(\Delta Y_{i P}\right)^{2} \cdot \cos ^{2}\left(A z_{i P}^{O}\right)+\left(\Delta X_{i P}\right)^{2} \cdot \operatorname{tg}\left(\beta_{i P}^{O}\right)+\left(\Delta Y_{i P}\right)^{2} \cdot \operatorname{tg}^{2}\left(\beta_{i P}^{O}\right) \\
\left(d_{i P}^{\prime O}\right)^{2}=\left(X_{P}-X_{i}\right)^{2} \cdot \operatorname{sen}^{2}\left(A z_{i P}^{O}\right)+2 \cdot\left(X_{P}-X_{i}\right) \cdot\left(Y_{P}-Y_{i}\right) \cdot \operatorname{sen}\left(A z_{i P}^{O}\right) \cdot \cos \left(A z_{i P}^{O}\right)+ \\
+\left(Y_{P}-Y_{i}\right)^{2} \cdot \cos ^{2}\left(A z_{i P}^{O}\right)+\left(X_{P}-X_{i}\right)^{2} \cdot \operatorname{tg}\left(\beta_{i P}^{O}\right)+\left(Y_{P}-Y_{i}\right)^{2} \cdot \operatorname{tg}^{2}\left(\beta_{i P}^{O}\right) \\
\left(d_{i P}^{\prime O}\right)^{2}=\left(X_{P}^{2}-2 \cdot X_{P} \cdot X_{i} \cdot X_{i}^{2}\right) \cdot \operatorname{sen}^{2}\left(A z_{i P}^{O}\right)+2 \cdot X_{P} \cdot Y_{P} \cdot \operatorname{sen}\left(A z_{i P}^{O}\right) \cdot \cos \left(A z_{i P}^{O}\right)- \\
+2 \cdot X_{P} \cdot Y_{i} \cdot \operatorname{sen}\left(A z_{i P}^{O}\right) \cdot \cos \left(A z_{i P}^{O}\right)-2 \cdot X_{i} \cdot Y_{P} \cdot \operatorname{sen}\left(A z_{i P}^{O}\right) \cdot \cos \left(A z_{i P}^{O}\right)+ \\
Y_{i} \cdot \operatorname{sen}\left(A z_{i P}^{O}\right) \cdot \cos \left(A z_{i P}^{O}\right)+\left(Y_{P}^{2}-2 \cdot Y_{P} \cdot Y_{i} \cdot Y_{i}^{2}\right) \cdot \cos ^{2}\left(A z_{i P}^{O}\right)+
\end{gathered}
$$




$$
\begin{gathered}
+\left(X_{P}^{2}-2 \cdot X_{P} \cdot X_{i} \cdot X_{i}^{2}\right) \cdot \operatorname{tg}^{2}\left(\beta_{i P}^{O}\right)+\left(Y_{P}^{2}-2 \cdot Y_{P} \cdot Y_{i} \cdot Y_{i}^{2}\right) \cdot \operatorname{tg}^{2}\left(\beta_{i P}^{O}\right) \\
\left(d_{i P}^{\prime O}\right)^{2}=\left(X_{P}-X_{i}\right)^{2} \cdot \operatorname{sen}^{2}\left(A z_{i P}^{O}\right)+2 \cdot\left(X_{P}-X_{i}\right) \cdot\left(Y_{P}-Y_{i}\right) \cdot \operatorname{sen}\left(A z_{i P}^{O}\right) \cdot \cos \left(A z_{i P}^{O}\right)+ \\
+\left(Y_{P}-Y_{i}\right)^{2} \cdot \cos ^{2}\left(A z_{i P}^{O}\right)+\left(X_{P}-X_{i}\right)^{2} \cdot \operatorname{tg}\left(\beta_{i P}^{O}\right)+\left(Y_{P}-Y_{i}\right)^{2} \cdot \operatorname{tg}^{2}\left(\beta_{i P}^{O}\right) \\
\left(d_{i P}^{\prime O}\right)^{2}=\left(\operatorname{sen}^{2}\left(A z_{i P}^{O}\right)+t^{2}\left(\beta_{i P}^{O}\right)\right) \cdot X_{P}^{2}+\left(-2 \cdot X_{i} \cdot \operatorname{sen}^{2}\left(A z_{i P}^{O}\right)-2 \cdot Y_{i} \cdot \operatorname{sen}\left(A z_{i P}^{O}\right) \cdot \cos \left(A z_{i P}^{O}\right)-\right. \\
\left.-2 \cdot X_{i} \cdot \operatorname{tg}^{2}\left(\beta_{i P}^{O}\right)\right) \cdot X_{P}+\left(-2 \cdot X_{i} \cdot \operatorname{sen}\left(A z_{i P}^{O}\right) \cdot \cos ^{2}\left(A z_{i P}^{O}\right)-2 \cdot Y_{i} \cdot \cos ^{2}\left(A z_{i P}^{O}\right)-2 \cdot Y_{i} \cdot \operatorname{tg}^{2}\left(\beta_{i P}^{O}\right)\right) \cdot Y_{P}+ \\
+\left(2 \cdot \operatorname{sen}\left(A z_{i P}^{O}\right) \cdot \cos \left(A z_{i P}^{O}\right)\right) \cdot X_{i} \cdot Y_{P}+\left(X_{i}^{2} \cdot \operatorname{sen}^{2}\left(A z_{i P}^{O}\right)+2 \cdot X_{i} \cdot Y_{i} \cdot \operatorname{sen}\left(A z_{i P}^{O}\right) \cdot \cos \left(A z_{i P}^{O}\right)+\right. \\
\left.+Y_{i}^{2} \cdot \cos ^{2}\left(A z_{i P}^{O}\right)+X_{i}^{2} \cdot \operatorname{tg}^{2}\left(\beta_{i P}^{O}\right)+Y_{i}^{2} \cdot \operatorname{tg}^{2}\left(\beta_{i P}^{O}\right)\right)+\left(\cos ^{2}\left(A z_{i P}^{O}\right)+\operatorname{tg}^{2}\left(\beta_{i P}^{O}\right)\right) \cdot Y_{P}^{2}
\end{gathered}
$$

Para facilitar o desenvolvimento do modelo matemático, serão definidos os seguintes parâmetros calculados, apresentados pelas equações (7.11 a 7.16),

$$
\begin{gathered}
A_{i p}=\operatorname{sen}^{2}\left(A z_{i P}^{O}\right)+\operatorname{tg}^{2}\left(\beta_{i P}^{O}\right) \\
B_{i P}=-2 \cdot X_{i} \cdot \operatorname{sen}^{2}\left(A z_{i P}^{O}\right)-2 \cdot Y_{i} \cdot \operatorname{sen}\left(A z_{i P}^{O}\right) \cdot \cos \left(A z_{i P}^{O}\right)-2 \cdot X_{i} \cdot \operatorname{tg}^{2}\left(\beta_{i P}^{O}\right) \\
C_{i P}=-2 \cdot X_{i} \cdot \operatorname{sen}\left(A z_{i P}^{O}\right) \cdot \cos \left(A z_{i P}^{O}\right)-2 \cdot Y_{i} \cdot \cos ^{2}\left(A z_{i P}^{O}\right)-2 \cdot Y_{i} \cdot \operatorname{tg}^{2}\left(\beta_{i P}^{O}\right) \\
D_{i P}=2 \cdot \operatorname{sen}\left(A z_{i P}^{O}\right) \cdot \cos \left(A z_{i P}^{O}\right) \\
E_{i P}=\cos ^{2}\left(A z_{i P}^{O}\right)+\operatorname{tg}^{2}\left(\beta_{i P}^{O}\right) \\
F_{i P}=X_{i}^{2} \cdot \operatorname{sen}^{2}\left(A z_{i P}^{O}\right)+2 \cdot X_{i} \cdot Y_{i} \cdot \operatorname{sen}\left(A z_{i P}^{O}\right) \cdot \cos \left(A z_{i P}^{O}\right)+
\end{gathered}
$$




$$
+Y_{i}^{2} \cdot \cos ^{2}\left(A z_{i P}^{O}\right)+X_{i}^{2} \cdot \operatorname{tg}^{2}\left(\beta_{i P}^{O}\right)+Y_{i}^{2} \cdot \operatorname{tg}^{2}\left(\beta_{i P}^{O}\right)
$$

Substituindo os parâmetros calculados acima, na equação (7.10), obtém-se,

$$
\left(d_{i P}^{\prime O}\right)^{2}=A_{i P} \cdot X_{P}^{2}+B_{i P} \cdot X_{P}+C_{i P} \cdot Y_{P}+D_{i P} \cdot X_{P} \cdot Y_{P}+E_{i P} \cdot Y_{P}^{2}+F_{i P}
$$

E reagrupando os termos, tem-se,

$$
\left(d_{i P}^{\prime O}\right)^{2}=A_{i P} \cdot X_{P}^{2}+E_{i P} \cdot Y_{P}^{2}+B_{i P} \cdot X_{P}+C_{i P} \cdot Y_{P}+D_{i P} \cdot X_{P} \cdot Y_{P}+F_{i P}
$$

Assim, para determinarmos as coordenadas $X_{P}$ e $Y_{P}$ do alvo, por meio das estações $i=1 \mathrm{e}$ $i=2$, deve-se resolver o seguinte sistema não linear,

$$
\left\{\begin{array}{l}
\left(d_{1 P}^{\prime O}\right)^{2}=A_{1 P} \cdot X_{P}^{2}+E_{1 P} \cdot Y_{P}^{2}+B_{1 P} \cdot X_{P}+C_{1 P} \cdot Y_{P}+D_{1 P} \cdot X_{P} \cdot Y_{P}+F_{1 P} \\
\left(d_{2 P}^{\prime O}\right)^{2}=A_{2 P} \cdot X_{P}^{2}+E_{2 P} \cdot Y_{P}^{2}+B_{2 P} \cdot X_{P}+C_{2 P} \cdot Y_{P}+D_{2 P} \cdot X_{P} \cdot Y_{P}+F_{2 P}
\end{array}\right.
$$

A resolução deste sistema não linear é realizada via Método de Newton Generalizado, que consiste em gerar, a partir de uma aproximação inicial para $X_{P}$ e $Y_{P}$, a qual deve ser escolhida, de modo a respeitar o critério de convergência do referido método (BURDEN; FAIRES; BURDEN, 2016). Esta aproximação inicial, é denotada por,

$$
P^{0}=\left[\begin{array}{c}
X_{P}^{0} \\
Y_{P}^{0}
\end{array}\right]
$$

De forma iterativa, gera-se uma sequência de soluções aproximadas,

$$
P^{k}=\left[\begin{array}{c}
X_{P}^{k} \\
Y_{P}^{k}
\end{array}\right]
$$

Onde, $k$ é o número natural representativo da ordem de iteração. Após iterações sucessivas, utilizando o critério de parada dados pelo valor absoluto das funções $f_{i}$, obtém-se o a melhor aproximação das coordenadas reais do alvo (DEMIDOVICH, 1981).

Notar que este procedimento pode ser generalizado para $n$ estações totais robóticas, tomadas duas a duas. De forma análoga, nesta tese, o procedimento foi realizado para três estações, considerando uma combinação aleatória simples $C_{3,2}$, isto é, $i=1$ e $i=2, i=1$ e $i=3$, $i=2$ e $i=3$. Ressalta-se que, as coordenadas espaciais do alvo, após os ajustamentos das observações, serão obtidas por média aritmética simples entre todas as combinações realizadas. Para efeito de apresentação, será considerado o caso particular $i=1,2$, sendo que os demais 
casos da combinação seguem de forma análoga. De acordo com o desenvolvimento previsto pelo Método de Newton Generalizado, definem-se as seguintes funções a serem linearizadas,

$$
f_{i}\left(X_{P}, Y_{P}\right)=A_{i P} \cdot X_{P}^{2}+E_{i P} \cdot Y_{P}^{2}+B_{i P} \cdot X_{P}+C_{i P} \cdot Y_{P}+D_{i P} \cdot X_{P} \cdot Y_{P}+F_{i P}-\left(d_{i P}^{\prime O}\right)^{2}
$$

Resolver o sistema não linear é equivalente a determinar os zeros das funções $f_{i}, i=1,2$, e uma melhor aproximação para $X_{P}$ e $Y_{P}$ ocorre, quando $f_{i}, i=1,2$ estão o mais próximo possível de zero.

Definindo,

$$
F\left(X_{P}, Y_{P}\right)=\left[\begin{array}{l}
f_{1}\left(X_{P}, Y_{P}\right) \\
f_{2}\left(X_{P}, Y_{P}\right)
\end{array}\right]
$$

Considerando abaixo a Matriz Jacobiana de $F$,

$$
J_{F}\left(X_{P}, Y_{P}\right)=\left[\begin{array}{ll}
\frac{\partial f_{1}}{\partial X_{P}} & \frac{\partial f_{1}}{\partial Y_{P}} \\
\frac{\partial f_{2}}{\partial X_{P}} & \frac{\partial f_{2}}{\partial Y_{P}}
\end{array}\right]
$$

Sabendo-se que,

$$
f_{1}\left(X_{P}, Y_{P}\right)=A_{1 P} \cdot X_{P}^{2}+E_{1 P} \cdot Y_{P}^{2}+B_{1 P} \cdot X_{P}+C_{1 P} \cdot Y_{P}+D_{1 P} \cdot X_{P} \cdot Y_{P}+F_{1 P}-\left(d_{1 P}^{\prime O}\right)^{2}
$$

$$
f_{2}\left(X_{P}, Y_{P}\right)=A_{2 P} \cdot X_{P}^{2}+E_{2 P} \cdot Y_{P}^{2}+B_{2 P} \cdot X_{P}+C_{2 P} \cdot Y_{P}+D_{2 P} \cdot X_{P} \cdot Y_{P}+F_{2 P}-\left(d_{2 P}^{\prime O}\right)^{2}
$$

Obtém-se as seguintes derivadas parciais em relação às variáveis $\left(X_{P}, Y_{P}\right)$,

$$
\begin{array}{ll}
\frac{\partial f_{1}}{\partial X_{P}}=2 A_{1 P} \cdot X_{P}+B_{1 P}+D_{1 P} \cdot Y_{P} & \frac{\partial f_{1}}{\partial Y_{P}}=2 E_{1 P} \cdot Y_{P}+C_{1 P}+D_{1 P} \cdot X_{P} \\
\frac{\partial f_{2}}{\partial X_{P}}=2 A_{2 P} \cdot X_{P}+B_{2 P}+D_{2 P} \cdot Y_{P} & \frac{\partial f_{2}}{\partial Y_{P}}=2 E_{2 P} \cdot Y_{P}+C_{2 P}+D_{2 P} \cdot X_{P}
\end{array}
$$

Logo, o sistema linearizado fica na forma,

$$
\left[\begin{array}{ll}
\frac{\partial f_{1}}{\partial X_{P}} & \frac{\partial f_{1}}{\partial Y_{P}} \\
\frac{\partial f_{2}}{\partial X_{P}} & \frac{\partial f_{2}}{\partial Y_{P}}
\end{array}\right] \cdot\left[\begin{array}{c}
X_{P}-X_{P}^{0} \\
Y_{P}-Y_{P}^{0}
\end{array}\right]=\left[\begin{array}{l}
-f_{1}\left(X_{P}, Y_{P}\right) \\
-f_{2}\left(X_{P}, Y_{P}\right)
\end{array}\right]
$$

Portanto, 


$$
\left[\begin{array}{c}
X_{P}-X_{P}^{0} \\
Y_{P}-Y_{P}^{0}
\end{array}\right]=J_{F}^{-1} \cdot\left[\begin{array}{l}
-f_{1}\left(X_{P}, Y_{P}\right) \\
-f_{2}\left(X_{P}, Y_{P}\right)
\end{array}\right]
$$

Dessa forma, se for escolhida uma aproximação inicial $P^{0}=\left[\begin{array}{c}X_{P}^{0} \\ Y_{P}^{0}\end{array}\right]$ para as coordenadas do alvo, pode-se gerar uma sequência $P^{0}, P^{1}, P^{2}, P^{K} \ldots, P^{K+1}$ que converge para as coordenadas reais do alvo. Tal sequência pode ser gerada da seguinte forma,

$$
P^{k+1}=P^{k}-\left(J_{F}\left(P^{k}\right)\right)^{-1} * F\left(P^{k}\right)
$$

Devido ao fato de o método ser iterativo, é necessário adotar um critério de parada, o qual foi considerado, nesta tese, por ||$F\left(P^{k}\right) \|=\max \left|f_{i}\left(P^{k}\right)\right|<\epsilon$, sendo $\epsilon$ - tolerância (DEMIDOVICH, 1981).

Após o processo iterativo, obtiveram-se as coordenadas $\left(X_{1,2, P}, Y_{1,2, P}\right)$ do ponto $P$ para a combinação específica $i=1$ e 2 . Já para o cálculo da coordenada $Z_{1,2, P}$, as equações (7.22) a (7.25) originaram-se da Figura 7.2.

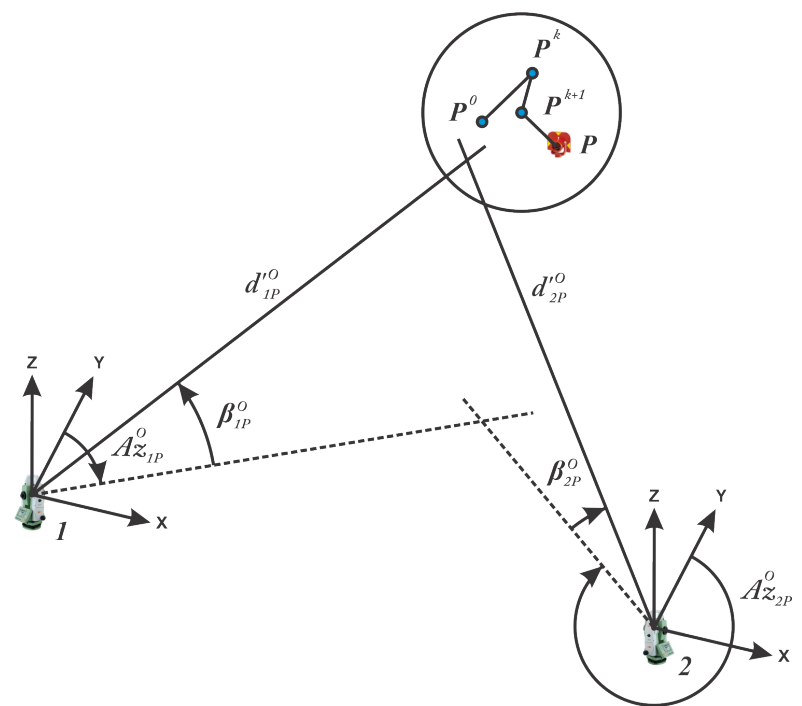

Figura 7.2: Representação do processo iterativo, com aproximações sucessivas para duas estações totais robóticas.

$$
\begin{gathered}
\Delta Z_{1 P}=\sqrt{\left(X_{P}-X_{P}^{0}\right)^{2}+\left(Y_{P}-Y_{P}^{0}\right)^{2}} \cdot \operatorname{tg}\left(\beta_{1 P}^{O}\right) \\
\Delta Z_{2 P}=\sqrt{\left(X_{P}-X_{P}^{0}\right)^{2}+\left(Y_{P}-Y_{P}^{0}\right)^{2}} \cdot \operatorname{tg}\left(\beta_{2 P}^{O}\right) \\
\Delta Z_{1,2, P}=\frac{\Delta Z_{1 P}+\Delta Z_{2 P}}{2}
\end{gathered}
$$




$$
Z_{1,2, P}=\Delta Z_{1,2, P}+Z_{P}^{0}
$$

Ressalta-se, ainda, que quando se está em uma vizinhança de $P^{0}$, podem-se considerar os mesmos ângulos verticais de altura para ambas estações.

Têm-se assim as coordenadas $\left(X_{1,2, P}, Y_{1,2, P}, Z_{1,2, P}\right)$ do alvo $P$ para esta combinação específica. Ao se repetir o procedimento para os casos $i=1$ e 3 e $i=2$ e 3, geram-se respectivamente outras coordenadas, $\left(X_{1,3, P}, Y_{1,3, P}, Z_{1,3, P}\right)$ e $\left(X_{2,3, P}, Y_{2,3, P}, Z_{2,3, P}\right)$ do alvo. Portanto, considera-se a coordenada final do alvo $P$, como sendo a média aritmética simples entre os três ternos de coordenadas obtidas.

$$
\begin{gathered}
\bar{X}_{P}=\frac{X_{1,2, P}+X_{1,3, P}+X_{2,3, P}}{3} \\
\bar{Y}_{P}=\frac{Y_{1,2, P}+Y_{1,3, P}+Y_{2,3, P}}{3} \\
\bar{Z}_{P}=\frac{Z_{1,2, P}+Z_{1,3, P}+Z_{2,3, P}}{3}
\end{gathered}
$$

Por fim, aplica-se o Método dos Mínimos Quadrados para o ajustamento das observações. As equações de erros para o azimute, o ângulo vertical de altura e a distância inclinada são indicadas a seguir, e as equações, respectivamente, (7.1), (7.2) e (7.4) expostas acima, repetemse para facilitar a apresentação da modelagem,

$$
\begin{gathered}
A z_{i P}^{C}=\operatorname{arctg}\left(\frac{\Delta X}{\Delta X}\right)=\operatorname{arctg}\left(\frac{X_{P}-X_{i}}{Y_{P}-Y_{i}}\right) \\
\beta_{i P}^{C}=\operatorname{arctg}\left(\frac{\Delta X}{\sqrt{(\Delta X)^{2}+(\Delta Y)^{2}}}\right)=\operatorname{arctg}\left(\frac{Z_{P}-Z_{i}}{\sqrt{\left(X_{P}-X_{i}\right)^{2}+\left(Y_{P}-Y_{i}\right)^{2}}}\right) \\
d_{i P}^{\prime C}=\sqrt{\left[\left(X_{P}-X_{i}\right) \operatorname{sen}\left(A z_{i P}\right)+\left(Y_{P}-Y_{i}\right) \cos \left(A z_{i P}\right)\right]^{2}+\left[(\Delta X)^{2}+(\Delta Y)^{2}\right] \cdot \operatorname{tg}^{2}\left(\beta_{i P}^{C}\right)}
\end{gathered}
$$

Dessa forma, por meio de relações geométricas obtêm-se os parâmetros calculados para a observação $A z_{i P}^{O}$,

$$
k_{i P}=\frac{\cos A z_{i P}^{C}}{d_{i P}^{\prime C}} \quad m_{i P}=-\frac{\operatorname{sen} A z_{i P}^{C}}{d_{i P}^{\prime C}} \quad n_{i P}=0
$$

E, consequentemente, a equação de erro correspondente ao azimute observado,

$$
v_{A z_{i P}^{O}}=k_{i P} \cdot \delta x+m_{i P} \cdot \delta y+n_{i P} \cdot \delta z-\left(A z_{i P}^{O}-A z_{i P}^{C}\right)
$$


7.1. Método iterativo de aproximações sucessivas, para determinação de coordenadas espaciais, considerando duas estações totais robóticas

Já, a partir de relações analíticas com base nas equações (7.30) e (7.31) geram-se as equações de erros para as observações $\beta_{i P}^{O}$ e $d_{i P}^{\prime O}$,

$$
\begin{aligned}
& v_{\beta_{i P}^{O}}=\frac{\partial \beta_{i P}^{C}}{\partial X_{P}} \delta X_{P}+\frac{\partial \beta_{i P}^{C}}{\partial Y_{P}} \delta Y_{P}+\frac{\partial \beta_{i P}^{C}}{\partial Z_{P}} \delta Z_{P}-\left(\beta_{i P}^{O}-\beta_{i P}^{C}\right) \\
& v_{d_{i P}^{\prime O}}=\frac{\partial d_{i P}^{\prime C}}{\partial X_{P}} \delta X_{P}+\frac{\partial d_{i P}^{\prime C}}{\partial Y_{P}} \delta Y_{P}+\frac{\partial d_{i P}^{\prime C}}{\partial Z_{P}} \delta Z_{P}-\left(d_{i P}^{\prime O}-d_{i P}^{\prime C}\right)
\end{aligned}
$$

Em seguida, apresentam-se as derivadas parciais das respectivas funções em relação aos valores aproximados das incógnitas,

$$
\begin{gathered}
\frac{\partial \beta_{i P}^{C}}{\partial X_{P}}=-\frac{\left(Z_{P}-Z_{i}\right)\left(X_{P}-X_{i}\right)}{\sqrt{\left(X_{P}-X_{i}\right)^{2}+\left(Y_{P}-Y_{i}\right)^{2}} \cdot d_{i P}^{\prime C}} \\
\frac{\partial \beta_{i P}^{C}}{\partial Y_{P}}=-\frac{\left(Z_{P}-Z_{i}\right)\left(Y_{P}-Y_{i}\right)}{\sqrt{\left(X_{P}-X_{i}\right)^{2}+\left(Y_{P}-Y_{i}\right)^{2}} \cdot d_{i P}^{\prime C}} \\
\frac{\partial \beta_{i P}^{C}}{\partial Z_{P}}=\frac{\sqrt{\left(X_{P}-X_{i}\right)^{2}+\left(Y_{P}-Y_{i}\right)^{2}}}{d_{i P}^{\prime C}} \\
\frac{\partial d_{i P}^{\prime C}}{\partial X_{P}}=\frac{\left[\left(X_{P}-X_{i}\right) \cdot \operatorname{sen}\left(A z_{i P}^{C}\right)+\left(Y_{P}-Y_{i}\right) \cdot \cos \left(A z_{i P}^{C}\right)\right] \cdot \operatorname{sen}\left(A z_{i P}^{C}\right)}{d_{i P}^{\prime C}}=\frac{\left[\left(X_{P}-X_{i}\right) \cdot \operatorname{sen}\left(A z_{i P}^{C}\right)+\left(Y_{P}-Y_{i}\right) \cdot \cos \left(A z_{i P}^{C}\right)\right] \cdot \cos \left(A z_{i P}^{C}\right)}{d_{i P}^{\prime C}} \\
\frac{\partial d_{i P}^{\prime C}}{\partial Z_{P}}=\frac{\left(Z_{P}-Z_{i}\right)}{d_{i P}^{\prime C}}
\end{gathered}
$$

Sendo,

$A z_{i P}^{O}$ - azimute medido da direção $i P$

$A z_{i P}^{C}$ - azimute calculado da direção $i P$

$\beta_{i P}^{O}$ - ângulo vertical de altura medido entre $i P$

$\beta_{i P}^{C}$ - ângulo vertical de altura calculado entre $i P$

$d_{i P}^{\prime O}$ - distância inclinada medida entre $i P$

$d_{i P}^{\prime C}$ - distância inclinada calculada entre $i P$

$\delta X_{P}, \delta Y_{P}, \delta Z_{P}$ - valor dos acréscimos para obtenção das coordenadas ajustadas

É importante ressaltar que o ajustamento das observações para esta modelagem pode ser realizado, com variadas combinações, devido ao fato da possibilidade de alguma das estações totais robóticas apresentar falha e se tornar inoperante por um determinado período de tempo. 
Para a situação em que uma das estações totais robóticas não seja considerada, deve-se utilizar a modelagem prevista para o caso particular entre as estações totais robóticas operantes.

Para exemplificação do ajustamento das observações, considera-se o caso de duas visadas partindo das estações totais robóticas $1 P$ e $2 P$, tem-se,

$$
V=A X-L
$$

Sendo,

$$
V=\left[\begin{array}{c}
v_{A z_{1 P}^{O}} \\
v_{\beta_{1 P}^{O}} \\
v_{d_{1 P}^{\prime O}} \\
v_{A z_{2 P}^{O}} \\
v_{\beta_{2 P}^{O}} \\
v_{d_{2 P}^{\prime}}
\end{array}\right] \quad A=\left[\begin{array}{lll}
k_{1 P} & m_{1 P} & n_{1 P} \\
\frac{\partial \beta_{1 P}^{C}}{\partial X_{P}} & \frac{\partial \beta_{1 P}^{C}}{\partial Y_{P}} & \frac{\partial \beta_{1 P}^{C}}{\partial Z_{P}} \\
\frac{\partial d_{1 P}^{\prime C}}{\partial X_{P}} & \frac{\partial d_{1 P}^{\prime C}}{\partial Y_{P}} & \frac{\partial d_{1 P}^{\prime}}{\partial Z_{P}} \\
k_{2 P} & m_{2 P} & n_{2 P} \\
\frac{\partial \beta_{2 P}^{C}}{\partial X_{P}} & \frac{\partial \beta_{2 P}^{C}}{\partial Y_{P}} & \frac{\partial \beta_{2 P}^{C}}{\partial Z_{P}} \\
\frac{\partial d_{2 P}^{\prime C}}{\partial X_{P}} & \frac{\partial d_{2 P}^{\prime C}}{\partial Y_{P}} & \frac{\partial d_{2 P}^{\prime C}}{\partial Z_{P}}
\end{array}\right] \quad X=\left[\begin{array}{c}
\delta X_{P} \\
\delta Y_{P} \\
\delta Z_{P}
\end{array}\right] \quad L=\left[\begin{array}{c}
A z_{1 P}^{O}-A z_{1 P}^{C} \\
\beta_{1 P}^{O}-\beta_{1 P}^{C} \\
d_{1 P}^{\prime O}-d_{1 P}^{\prime C} \\
A z_{2 P}^{O}-A z_{2 P}^{C} \\
\beta_{2 P}^{O}-\beta_{2 P}^{C} \\
d_{2 P}^{\prime O}-d_{2 P}^{\prime C}
\end{array}\right]
$$

De acordo com a seção 4.5, tem-se,

$$
X=\left(A^{T} P A\right)^{-1}\left(A^{T} P L\right)
$$

As precisões das incógnitas $X$ podem ser calculadas considerando as equações (4.22) e (4.23). Dessa forma, é possível determinar as precisões de cada incógnita $X_{P}, Y_{P}$ e $Z_{P}$.

$$
\sigma_{X_{P}}=\sqrt{\hat{\sigma}_{X_{P}}^{2}} \quad \sigma_{Y_{P}}=\sqrt{\hat{\sigma}_{Y_{P}}^{2}} \quad \sigma_{Z_{P}}=\sqrt{\hat{\sigma}_{Z_{P}}^{2}}
$$

E por fim, as precisões das coordenadas espaciais $\left(X_{P}, Y_{P}, Z_{P}\right)$.

$$
\sigma_{X_{P} Y_{P} Z_{P}}=\sqrt{\left(\sigma_{X_{P}}\right)^{2}+\left(\sigma_{Y_{P}}\right)^{2}+\left(\sigma_{Z_{P}}\right)^{2}}
$$

\subsection{Método iterativo de aproximações sucessivas, para determinação de coordenadas espaciais, considerando três estações totais robó- ticas}

Para esta modelagem matemática, como se consideram três estações totais robóticas, devese obter uma função que dependa dos parâmetros $A z_{i P}^{O}, \beta_{i P}^{O}$ e $d_{i P}^{\prime O}$ medidos e das variáveis $\left(X_{P}, Y_{P}, Z_{P}\right)$. Considerando a situação geométrica da Figura 6.12, para o caso de três estações, tem-se a equação (7.45) para a distância. 


$$
d_{i P}^{\prime O}=\sqrt{\left[\left(X_{P}-X_{i}\right) \operatorname{sen}\left(A z_{i P}^{O}\right)+\left(Y_{P}-Y_{i}\right) \cos \left(A z_{i P}^{O}\right)\right]^{2}+\left(Z_{P}-Z_{i}\right)^{2}}
$$

Sendo,

$i=1,2$ e 3

A partir do desenvolvimento do quadrado da soma e da diferença da equação (7.45) e, com o reagrupamento dos termos semelhantes, obtém-se a equação (7.46),

$$
\begin{array}{r}
\left(d_{i P}^{\prime}\right)^{2}=X_{P}^{2} \cdot \operatorname{sen}^{2}\left(A z_{i P}^{O}\right)+Y_{P}^{2} \cdot \cos ^{2}\left(A z_{i P}^{O}\right)+Z_{P}^{2}-2 \cdot X_{P} \cdot X_{i} \cdot \operatorname{sen}^{2}\left(A z_{i P}^{O}\right)+ \\
+2 \cdot X_{P} \cdot Y_{P} \cdot \operatorname{sen}\left(A z_{i P}^{O}\right) \cdot \cos \left(A z_{i P}^{O}\right)-2 \cdot X_{P} \cdot Y_{i} \cdot \operatorname{sen}\left(A z_{i P}^{O}\right) \cdot \cos \left(A z_{i P}^{O}\right)- \\
-2 \cdot X_{i} \cdot Y_{P} \cdot \operatorname{sen}\left(A z_{i P}^{O}\right) \cdot \cos \left(A z_{i P}^{O}\right)+2 \cdot X_{i} \cdot Y_{i} \cdot \operatorname{sen}\left(A z_{i P}^{O}\right) \cdot \cos \left(A z_{i P}^{O}\right)- \\
-2 \cdot Y_{P} \cdot Y_{i} \cdot \cos ^{2}\left(A z_{i P}^{O}\right)-2 \cdot Z_{P} \cdot Z_{i}+X_{i}^{2} \cdot \operatorname{sen}^{2}\left(A z_{i P}^{O}\right)+Y_{i}^{2} \cdot \cos ^{2}\left(A z_{i P}^{O}\right)+Z_{i}^{2}
\end{array}
$$

Tal equação é denotada por,

$$
\begin{gathered}
\mathcal{A}_{i P}=\operatorname{sen}^{2}\left(A z_{i P}^{O}\right) \\
\mathcal{B}_{i P}=\cos ^{2}\left(A z_{i P}^{O}\right) \\
\mathcal{C}_{i P}=\operatorname{sen}\left(A z_{i P}^{O}\right) \cdot \cos \left(A z_{i P}^{O}\right) \\
\mathcal{D}_{i P}=2 \cdot X_{i} \cdot Y_{i} \cdot \operatorname{sen}\left(A z_{i P}^{O}\right) \cdot \cos \left(A z_{i P}^{O}\right)+\mathcal{A}_{i P} \cdot X_{i}^{2}+\mathcal{B}_{i P} \cdot Y_{i}^{2}+Z_{i}^{2}
\end{gathered}
$$

Reescrevendo $\left(d_{i P}^{\prime}\right)^{2}$, temos a equação (7.51),

$$
\begin{gathered}
\left(d_{i P}^{\prime O}\right)^{2}=\mathcal{A}_{i P} \cdot X_{P}^{2}+\mathcal{B}_{i P} \cdot Y_{P}^{2}+Z_{P}^{2}-2 \cdot \mathcal{A}_{i P} \cdot X_{P} \cdot X_{i}+ \\
+2 \cdot C_{i P} \cdot X_{P} \cdot Y_{P}-2 \cdot C_{i P} \cdot X_{P} \cdot Y_{i}-2 \cdot \mathcal{C}_{i P} \cdot X_{i} \cdot Y_{P}- \\
-2 \cdot \mathcal{B}_{i P} \cdot Y_{P} \cdot Y_{i}-2 \cdot Z_{P} \cdot Z_{i}+\mathcal{D}_{i P}
\end{gathered}
$$


Logo, para se determinar as coordenadas $\left(X_{P}, Y_{P}, Z_{P}\right)$, deve-se resolver o seguinte sistema não linear,

$$
\left\{\begin{array}{l}
\left(d_{1 P}^{\prime O}\right)^{2}-\mathcal{D}_{1 P}=\mathcal{A}_{1 P} X_{P}^{2}+\mathcal{B}_{1 P} Y_{P}^{2}+Z_{P}^{2}-2 \mathcal{A}_{1 P} X_{P} X_{1}+2 C_{1 P}\left(X_{P} Y_{P}-X_{P} Y_{1}-X_{1} Y_{P}\right)-2 \mathcal{B}_{1 P} Y_{P} Y_{1}-2 Z_{P} Z_{1} \\
\left(d_{2 P}^{\prime O}\right)^{2}-\mathcal{D}_{2 P}=\mathcal{A}_{2 P} X_{P}^{2}+\mathcal{B}_{2 P} Y_{P}^{2}+Z_{P}^{2}-2 \mathcal{A}_{2 P} X_{P} X_{2}+2 C_{2 P}\left(X_{P} Y_{P}-X_{P} Y_{2}-X_{2} Y_{P}\right)-2 \mathcal{B}_{2 P} Y_{P} Y_{2}-2 Z_{P} Z_{2} \\
\left(d_{3 P}^{\prime O}\right)^{2}-\mathcal{D}_{3 P}=\mathcal{A}_{3 P} X_{P}^{2}+\mathcal{B}_{3 P} Y_{P}^{2}+Z_{P}^{2}-2 \mathcal{A}_{3 P} X_{P} X_{3}+2 C_{3 P}\left(X_{P} Y_{P}-X_{P} Y_{3}-X_{3} Y_{P}\right)-2 \mathcal{B}_{3 P} Y_{P} Y_{3}-2 Z_{P} Z_{3}
\end{array}\right.
$$

Ressalta-se que a resolução do sistema não linear, acima, é equivalente a determinar os zeros da função $F: \mathbb{R}^{3} \rightarrow \mathbb{R}^{3}$, definida da seguinte forma,

$$
F\left(X_{P}, Y_{P}, Z_{P}\right)=\left[\begin{array}{c}
f_{1}\left(X_{P}, Y_{P}, Z_{P}\right) \\
f_{2}\left(X_{P}, Y_{P}, Z_{P}\right) \\
f_{3}\left(X_{P}, Y_{P}, Z_{P}\right)
\end{array}\right]
$$

Sendo,

$$
\begin{gathered}
f_{i}\left(X_{P}, Y_{P}, Z_{P}\right)=-\left(d_{i P}^{\prime O}\right)^{2}+\mathcal{D}_{i P}+\mathcal{A}_{i P} X_{P}^{2}+\mathcal{B}_{i P} Y_{P}^{2}+Z_{P}^{2}-2 \mathcal{A}_{i P} X_{P} X_{i}+ \\
+2 C_{i P}\left(X_{P} Y_{P}-X_{P} Y_{i}-X_{i} Y_{P}\right)-2 \mathcal{B}_{i P} Y_{P} Y_{i}-2 Z_{P} Z_{i}
\end{gathered}
$$

Para isso, foi utilizado o Método de Newton Generalizado, que consiste em resolver o seguinte sistema linearizado,

$$
J_{F}\left(P^{k}\right) * P^{k}=-F\left(P^{k}\right)
$$

Com,

$$
P^{k}=\left[\begin{array}{c}
X_{P}^{k} \\
Y_{P}^{k} \\
Z_{P}^{k}
\end{array}\right]
$$

Com $k=0,1,2, \ldots$, sendo o número de iterações até a condição de parada estar satisfeita, em conformidade com Demidovich (1981), e a matriz Jacobiana dada por,

$$
J_{F}=\left[\begin{array}{lll}
\frac{\partial f_{1}}{\partial X_{P}} & \frac{\partial f_{1}}{\partial Y_{P}} & \frac{\partial f_{1}}{\partial Z_{P}} \\
\frac{\partial f_{2}}{\partial X_{P}} & \frac{\partial f_{2}}{\partial Y_{P}} & \frac{\partial f_{2}}{\partial Z_{P}} \\
\frac{\partial f_{3}}{\partial X_{P}} & \frac{\partial f_{3}}{\partial Y_{P}} & \frac{\partial f_{3}}{\partial Z_{P}}
\end{array}\right]
$$

Logo, se for escolhido uma aproximação inicial $P^{0}=\left(X_{P}^{0}, Y_{P}^{0}, Z_{P}^{0}\right)$ para as coordenadas do alvo de modo que a matriz Jacobiana seja invertível, pode-se gerar uma sequência $P^{0}, P^{1}, P^{2}, \ldots$, que converge para as coordenadas reais do alvo, e que pode ser descrita da seguinte forma,

$$
P^{k+1}=P^{k}-\left(J_{F}\left(P^{k}\right)\right)^{-1} * F\left(P^{k}\right)
$$


7.2. Método iterativo de aproximações sucessivas, para determinação de coordenadas espaciais, considerando três estações totais robóticas

Dessa maneira, segue abaixo o cálculo das derivadas parciais que compõem a matriz Jacobiana,

$$
\begin{aligned}
& \frac{\partial f_{1}}{\partial X_{P}}=2 \mathcal{A}_{1 P} X_{P}-2 \mathcal{A}_{1 P} X_{1}+2 C_{1 P}\left(Y_{P}-Y_{1}\right) \quad \frac{\partial f_{1}}{\partial Y_{P}}=2 \mathcal{B}_{1 P} Y_{P}-2 \mathcal{B}_{1 P} Y_{1}+2 C_{1 P}\left(X_{P}-X_{1}\right) \\
& \frac{\partial f_{1}}{\partial Z_{P}}=2\left(Z_{P}-Z_{1}\right) \sec ^{2}\left(\beta_{1 P}\right) \sqrt{\left(X_{P}-X_{1}\right)^{2}+\left(Y_{P}-Y_{1}\right)^{2}} \\
& \frac{\partial f_{2}}{\partial X_{P}}=2 \mathcal{A}_{2 P} X_{P}-2 \mathcal{A}_{2 P} X_{2}+2 C_{2 P}\left(Y_{P}-Y_{2}\right) \quad \frac{\partial f_{2}}{\partial Y_{P}}=2 \mathcal{B}_{2 P} Y_{P}-2 \mathcal{B}_{2 P} Y_{2}+2 C_{2 P}\left(X_{P}-X_{2}\right) \\
& \frac{\partial f_{2}}{\partial Z_{P}}=2\left(Z_{P}-Z_{2}\right) \sec ^{2}\left(\beta_{2 P}\right) \sqrt{\left(X_{P}-X_{2}\right)^{2}+\left(Y_{P}-Y_{2}\right)^{2}} \\
& \frac{\partial f_{3}}{\partial X_{P}}=2 \mathcal{A}_{3 P} X_{P}-2 \mathcal{A}_{3 P} X_{3}+2 C_{3 P}\left(Y_{P}-Y_{3}\right) \quad \frac{\partial f_{3}}{\partial Y_{P}}=2 \mathcal{B}_{3 P} Y_{P}-2 \mathcal{B}_{3 P} Y_{3}+2 C_{3 P}\left(X_{P}-X_{3}\right) \\
& \frac{\partial f_{3}}{\partial Z_{P}}=2\left(Z_{P}-Z_{3}\right) \sec ^{2}\left(\beta_{3 P}\right) \sqrt{\left(X_{P}-X_{3}\right)^{2}+\left(Y_{P}-Y_{3}\right)^{2}}
\end{aligned}
$$

Assim, a Matriz Jacobiana fica da seguinte forma,

$$
\mathrm{J}_{F}=\left[\begin{array}{lll}
2 \mathcal{A}_{1 P} X_{P}^{k}-2 \mathcal{A}_{1 P} X_{1}+2 C_{1 P}\left(Y_{P}^{k}-Y_{1}\right) & 2 \mathcal{B}_{1 P} Y_{P}^{k}-2 \mathcal{B}_{1 P} Y_{1}+2 C_{1 P}\left(X_{P}^{k}-X_{1}\right) & 2\left(Z_{P}^{k}-Z_{1}\right) \sec ^{2}\left(\beta_{1 P}\right) \sqrt{\left(X_{P}^{k}-X_{1}\right)^{2}+\left(Y_{P}^{k}-Y_{1}\right)^{2}} \\
2 \mathcal{A}_{2 P} X_{P}^{k}-2 \mathcal{A}_{2 P} X_{2}+2 C_{2 P}\left(Y_{P}^{k}-Y_{2}\right) & 2 \mathcal{B}_{2 P} Y_{P}^{k}-2 \mathcal{B}_{2 P} Y_{2}+2 C_{2 P}\left(X_{P}^{k}-X_{2}\right) & 2\left(Z_{P}^{k}-Z_{2}\right) \sec ^{2}\left(\beta_{2 P}\right) \sqrt{\left(X_{P}^{k}-X_{2}\right)^{2}+\left(Y_{P}^{k}-Y_{2}\right)^{2}} \\
2 \mathcal{A}_{3 P} X_{P}^{k}-2 \mathcal{A}_{3 P} X_{3}+2 C_{3 P}\left(Y_{P}^{k}-Y_{3}\right) & 2 \mathcal{B}_{3 P} Y_{P}^{k}-2 \mathcal{B}_{3 P} Y_{3}+2 C_{3 P}\left(X_{P}^{k}-X_{3}\right) & 2\left(Z_{P}^{k}-Z_{3}\right) \sec ^{2}\left(\beta_{3 P}\right) \sqrt{\left(X_{P}^{k}-X_{3}\right)^{2}+\left(Y_{P}^{k}-Y_{3}\right)^{2}}
\end{array}\right]
$$

Ao entrar com os valores das coordenadas espaciais das estações de referência $\left(X_{1}, Y_{1}, Z_{1}\right)$, $\left(X_{2}, Y_{2}, Z_{2}\right),\left(X_{3}, Y_{3}, Z_{3}\right)$, é possível calcular o valor dos parâmetros $\mathcal{A}_{1 P}, \mathcal{B}_{1 P}, \mathcal{C}_{1 P}, \mathcal{A}_{2 P}, \mathcal{B}_{2 P}$, $C_{2 P}, \mathcal{A}_{3 P}, \mathcal{B}_{3 P}, C_{3 P}$. Em seguida, tem-se que adotar a aproximação inicial para o alvo, aqui considerado como o resultado método polar com média, que é denotado por $P^{0}=\left(X_{P}^{0}, Y_{P}^{0}, Z_{P}^{0}\right)$, nota que aqui $k=0$. Em seguida substituem-se todos esses valores na matriz Jacobiana acima e, assim, calcula-se a inversa desta matriz $\left(J_{F}\left(P^{k}\right)\right)^{-1}$. Além disso, deve-se calcular o valor de $F$ no ponto $P^{0}$. Em seguida, gera-se uma nova aproximação para as coordenadas do alvo $P^{1}=\left(X_{P}^{1}, Y_{P}^{1}, Z_{P}^{1}\right)$ pela fórmula,

$$
P^{1}=P^{0}-\left(J_{F}\left(P^{0}\right)\right)^{-1} * F\left(P^{0}\right)
$$

Pode-se observar que a fórmula acima é uma igualdade de matrizes, cujo processo deve ser repetido para $k=2,3$, e, assim, sucessivamente. A Figura 7.3 representa o processo iterativo com aproximações sucessivas para três estações totais robóticas. 


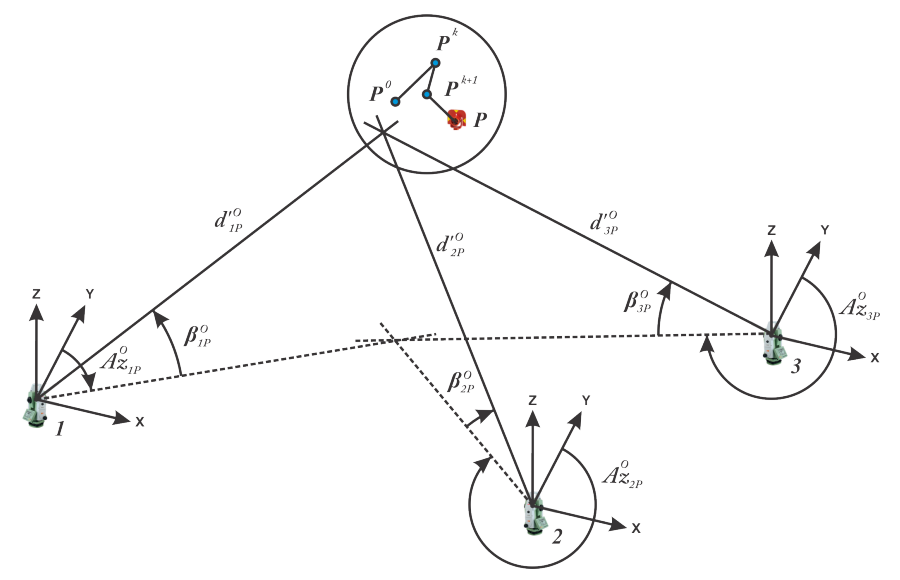

Figura 7.3: Representação do processo iterativo, com aproximações sucessivas para três estações totais robóticas.

Por fim, aplica-se o Método dos Mínimos Quadrados para o ajustamento das observações, sendo que as equações de erros para o azimute, o ângulo vertical de altura e a distância inclinada foram estabelecidas na forma analítica. Tais equações de erros podem ser encontradas, aplicando-se diretamente as funções trigonométricas entre os valores medidos e as incógnitas do problema. Têm-se, assim,

$$
\begin{gathered}
A z_{i P}^{C}=\operatorname{arctg}\left(\frac{X_{P}-X_{i}}{Y_{P}-Y_{i}}\right) \\
\beta_{i P}^{C}=\operatorname{arctg}\left(\frac{Z_{P}-Z_{i}}{\sqrt{\left(X_{P}-X_{i}\right)^{2}+\left(Y_{P}-Y_{i}\right)^{2}}}\right) \\
d_{i P}^{\prime C}=\sqrt{\left[\left(X_{P}-X_{i}\right) \operatorname{sen}\left(A z_{i P}^{C}\right)+\left(Y_{P}-Y_{i}\right) \cos \left(A z_{i P}^{C}\right)\right]^{2}+\left(Z_{P}-Z_{i}\right)^{2}}
\end{gathered}
$$

As equações de erros geradas pelas equações acima são iguais a:

$$
\begin{gathered}
v_{A z_{i P}^{O}}=\frac{\partial A z_{i P}^{C}}{\partial X_{P}} \delta X_{P}+\frac{\partial A z_{i P}^{C}}{\partial Y_{P}} \delta Y_{P}+\frac{\partial A z_{i P}^{C}}{\partial Z_{P}} \delta Z_{P}-\left(A z_{i P}^{O}-A z_{i P}^{C}\right) \\
v_{\beta_{i P}^{O}}=\frac{\partial \beta_{i P}^{C}}{\partial X_{P}} \delta X_{P}+\frac{\partial \beta_{i P}^{C}}{\partial Y_{P}} \delta Y_{P}+\frac{\partial \beta_{i P}^{C}}{\partial Z_{P}} \delta Z_{P}-\left(\beta_{i P}^{O}-\beta_{i P}^{C}\right) \\
v_{d_{i P}^{\prime C}}=\frac{\partial d_{i P}^{\prime C}}{\partial X_{P}} \delta X_{P}+\frac{\partial d_{i P}^{\prime C}}{\partial Y_{P}} \delta Y_{P}+\frac{\partial d_{i P}^{\prime C}}{\partial Z_{P}} \delta Z_{P}-\left(d_{i P}^{\prime O}-d_{i P}^{\prime C}\right)
\end{gathered}
$$

Sendo,

$$
\frac{\partial A z_{i P}^{C}}{\partial X_{P}}=\frac{\left(Y_{P}-Y_{i}\right)}{d_{i P}^{\prime C}}
$$




$$
\begin{gathered}
\frac{\partial A z_{i P}^{C}}{\partial Y_{P}}=-\frac{\left(X_{P}-X_{i}\right)}{d_{i P}^{\prime C}} \\
\frac{\partial A z_{i P}^{C}}{\partial Z_{P}}=0 \\
\frac{\partial \beta_{i P}^{C}}{\partial X_{P}}=-\frac{\left(Z_{P}-Z_{i}\right)\left(X_{P}-X_{i}\right)}{\sqrt{\left(X_{P}-X_{i}\right)^{2}+\left(Y_{P}-Y_{i}\right)^{2}} \cdot d_{i P}^{\prime C}} \\
\frac{\partial \beta_{i P}^{C}}{\partial Y_{P}}=-\frac{\left(Z_{P}-Z_{i}\right)\left(Y_{P}-Y_{i}\right)}{\sqrt{\left(X_{P}-X_{i}\right)^{2}+\left(Y_{P}-Y_{i}\right)^{2}} \cdot d_{i P}^{\prime C}} \\
\frac{\partial \beta_{i P}^{C}}{\partial Z_{P}}=\frac{\sqrt{\left(X_{P}-X_{i}\right)^{2}+\left(Y_{P}-Y_{i}\right)^{2}}}{d_{i P}^{\prime C}} \\
\frac{\partial d_{i P}^{\prime C}}{\partial Y_{P}}=\frac{\left[\left(X_{P}-X_{i}\right) \cdot \operatorname{sen}\left(A z_{i P}^{C}\right)+\left(Y_{P}-Y_{i}\right) \cdot \cos \left(A z_{i P}^{C}\right)\right] \cdot \operatorname{sen}\left(A z_{i P}^{C}\right)}{d_{i P}^{\prime C}} \\
\frac{\frac{\partial d_{i P}^{\prime C}}{\partial Z_{P}}=\frac{\left(Z_{P}-Z_{i}\right)}{d_{i P}^{\prime C}}}{d_{i P}^{\prime C}}
\end{gathered}
$$

Sendo,

$A z_{i P}^{O}$ - azimute medido da direção $i P$

$A z_{i P}^{C}$ - azimute calculado da direção $i P$

$\beta_{i P}^{O}$ - ângulo vertical de altura medido entre $i P$

$\beta_{i P}^{C}$ - ângulo vertical de altura calculado entre $i P$

$d_{i P}^{\prime O}$ - distância inclinada medida entre $i P$

$d_{i P}^{\prime C}$ - distância inclinada calculada entre $i P$

$\delta X_{P}, \delta Y_{P}, \delta Z_{P}$ - valor dos acréscimos para obtenção das coordenadas ajustadas

Nestas condições, considerando o caso de três visadas partindo das estações 1, 2 e 3, tem-se,

$$
V=A X-L
$$

Sendo, 


$$
V=\left[\begin{array}{c}
v_{A z_{1 P}^{O}} \\
v_{\beta_{1 P}^{O}} \\
v_{d_{1 P}^{\prime O}} \\
v_{A z_{2 P}^{O}} \\
v_{\beta_{2 P}^{O}} \\
v_{d_{2 P}^{\prime O}} \\
v_{A z_{3 P}^{O}} \\
v_{\beta_{3 P}}^{O} \\
v_{d_{3 P}^{\prime O}}
\end{array}\right] \quad A=\left[\begin{array}{lll}
\frac{\partial A z_{1 P}^{C}}{\partial X_{P}} & \frac{\partial A z_{1 P}^{C}}{\partial Y_{P}} & \frac{\partial A z_{1 P}^{C}}{\partial Z_{P}} \\
\frac{\partial \beta_{1 P}^{C}}{\partial X_{P}} & \frac{\partial \beta_{1 P}^{C}}{\partial Y_{P}} & \frac{\partial \beta_{1 P}^{C}}{\partial Z_{P}} \\
\frac{\partial d_{1 P}^{\prime}}{\partial X_{P}} & \frac{\partial d_{1 P}^{\prime C}}{\partial Y_{P}} & \frac{\partial d_{1 P}^{\prime}}{\partial Z_{P}} \\
\frac{\partial A z_{2 P}^{C}}{\partial X_{P}} & \frac{\partial A z_{2 P}^{C}}{\partial Y_{P}} & \frac{\partial A z_{2 P}^{C}}{\partial Z_{P}} \\
\frac{\partial \beta_{2 P}^{C}}{\partial X_{P}} & \frac{\partial \beta_{2 P}^{C}}{\partial Y_{P}} & \frac{\partial \beta_{2 P}^{C}}{\partial Z_{P}} \\
\frac{\partial d_{2 P}^{C}}{\partial X_{P}} & \frac{\partial d_{2 P}^{\prime C}}{\partial Y_{P}} & \frac{\partial d_{2 P}^{C}}{\partial Z_{P}} \\
\frac{\partial A z_{3 P}^{C}}{\partial X_{P}} & \frac{\partial A z_{3 P}^{C}}{\partial Y_{P}} & \frac{\partial A z_{3 P}^{C}}{\partial Z_{P}} \\
\frac{\partial \beta_{3 P}^{C}}{\partial X_{P}} & \frac{\partial \beta_{3 P}^{C}}{\partial Y_{P}} & \frac{\partial \beta_{3 P}^{C}}{\partial Z_{P}} \\
\frac{\partial d_{3 P}^{\prime C}}{\partial X_{P}} & \frac{\partial d_{3 P}^{\prime C}}{\partial Y_{P}} & \frac{\partial d_{3 P}^{\prime}}{\partial Z_{P}}
\end{array}\right] \quad X=\left[\begin{array}{c}
\delta X_{P} \\
\delta Y_{P} \\
\delta Z_{P}
\end{array}\right] \quad L=\left[\begin{array}{c}
A z_{1 P}^{O}-A z_{1 P}^{C} \\
\beta_{1 P}^{O}-\beta_{1 P}^{C} \\
d_{1 P}^{\prime O}-d_{1 P}^{\prime C} \\
A z_{2 P}^{O}-A z_{2 P}^{C} \\
\beta_{2 P}^{O}-\beta_{2 P}^{C} \\
d_{2 P}^{\prime O}-d_{2 P}^{\prime C} \\
A z_{3 P}^{O}-A z_{3 P}^{C} \\
\beta_{3 P}^{O}-\beta_{3 P}^{C} \\
d_{3 P}^{\prime O}-d_{3 P}^{\prime C}
\end{array}\right]
$$

De acordo com a seção 4.5 , tem-se,

$$
X=\left(A^{T} P A\right)^{-1}\left(A^{T} P L\right)
$$

As precisões das incógnitas $X$ podem ser calculadas considerando as equações (4.22) e (4.23).

Dessa forma, é possível determinar as precisões de cada incógnita $X_{P}, Y_{P}$ e $Z_{P}$.

$$
\sigma_{X_{P}}=\sqrt{\hat{\sigma}_{X_{P}}^{2}} \quad \sigma_{Y_{P}}=\sqrt{\hat{\sigma}_{Y_{P}}^{2}} \quad \sigma_{Z_{P}}=\sqrt{\hat{\sigma}_{Z_{P}}^{2}}
$$

E por fim, as precisões das coordenadas espaciais $\left(X_{P}, Y_{P}, Z_{P}\right)$.

$$
\sigma_{X_{P} Y_{P} Z_{P}}=\sqrt{\left(\sigma_{X_{P}}\right)^{2}+\left(\sigma_{Y_{P}}\right)^{2}+\left(\sigma_{Z_{P}}\right)^{2}}
$$




\section{Capítulo}

\section{SISTEMA DE MONITORAMENTO GEODÉSICO PROPOSTO}

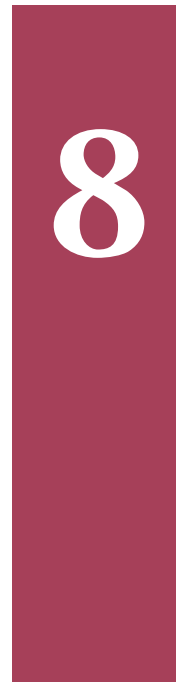

Neste capítulo, será apresentado inicialmente, o local utilizado para o desenvolvimento desta pesquisa; em seguida, a implantação da rede topográfica espacial, que se tornou referência absoluta para todo o sistema de coordenada local, definido para a realização de todas as medições e as determinações de coordenadas espaciais do processo de monitoramento.

Como citado anteriormente, o objetivo final de realizar medições utilizando técnicas geodésicas no monitoramento de estruturas, é determinar as coordenadas espaciais dos pontos na superfície da estrutura com deslocamento, em relação a um referencial fixo e estável. Assim, é possível, depois de observações periódicas, determinar as diferenças de coordenadas em épocas ou posições distintas e, consequentemente, os deslocamentos absolutos dos pontos.

Necessariamente, os valores previstos das grandezas a serem monitoradas, ou seja, os controles milimétricos na variação dos pontos têm que ser detectados pelas metodologias e instrumentos empregados. Dessa forma, não somente a precisão dos instrumentos, como também dos resultados obtidos, deverão estar adequadas aos deslocamentos milimétricos previstos para o estudo em questão.

Ainda, em relação ao padrão das precisões das observações, um aspecto importante está relacionado à escolha das técnicas e dos instrumentos utilizados. Nesse sentido, considerou-se como sendo zero, a altura de todos os instrumentos posicionados em cada base, para todos os procedimentos. Tal fato eliminou um erro inerente à determinação dos centros ópticos dos instrumentos, pois, sua altura sendo zero, automaticamente, todo o sistema está referenciado nos próprios centros ópticos dos instrumentos; mantendo, portanto, o mesmo referencial.

Os pontos da rede de referência e os alvos fixados no corpo estrutural podem ter suas posições controladas, por meio de séries de observações predeterminadas, utilizando o ajustamento 
de observações, no qual se emprega o MMQ em sua forma paramétrica. O ajustamento das observações e análises estatísticas são indispensáveis para trabalhos envolvendo mensuração, por fornecerem resultados confiáveis sobre as medidas obtidas em campo e sua modelagem em geral, o que expressa uma realidade física, com a melhor aproximação possível.

\subsection{Descrição do local de estudo}

O Campus da Universidade de São Paulo, em São Carlos, localizado na área 2, dispõe de amplo espaço físico, com extensas Áreas de Preservação Permanente (APP) e, por isso, com pouca densidade de edificações. Este local, portanto, foi escolhido, por possibilitar a espacialização de forma intervisível de todos os elementos envolvidos no processo de monitoramento e, assim, realizar as simulações necessárias para a investigação de uma auscultação estrutural com técnicas geodésicas, para validação do sistema proposto.

Como mostra a Figura 8.1, foram espacializadas seis bases para composição da rede topográfica espacial e um alvo com deslocamento controlado, para composição do objeto a ser monitorado.

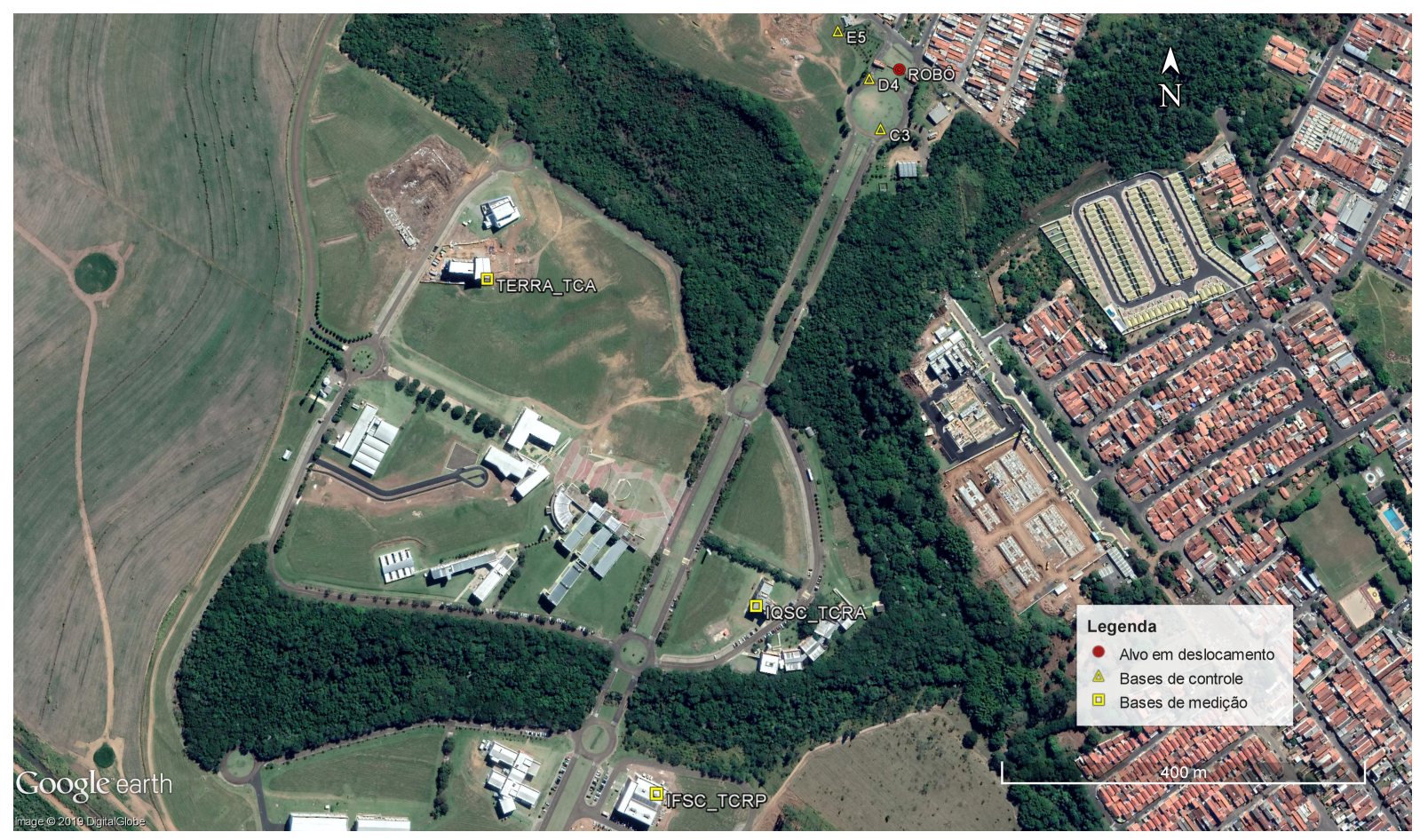

Figura 8.1: Localização e espacialização dos elementos no Campus 2 da USP São Carlos.

Para o processo de monitoramento, as bases desta rede foram divididas em três bases de medições e três de controle, ambas externas ao alvo.

No caso das bases de medições, uma foi instalada diretamente no perfil metálico do terceiro andar do edifício Polo Terra, outra foi instalada no terceiro andar do Instituto de Física de São Carlos (IFSC) e, a última, na marquise do Instituto de Química de São Carlos (IQSC). 
Já, em relação às bases de controle, uma foi instalada na rotatória da entrada Norte do Campus 2, uma no gramado da portaria desta entrada e, a última, na frente da central de segurança do mesmo Campus.

Por fim, o braço robótico uArm Swift Pro foi instalado em uma estrutura de concreto armado, alicerçada entre as portarias de entrada e de saída da área Norte do Campus 2.

Todos os elementos, envolvidos no processo de medição, foram posicionados de tal forma que estivessem o mais distante possível, porém intervisíveis.

\subsection{Subsistema de medição}

Em qualquer processo de medição, a adoção de um sistema referencial único simplifica a sistemática utilizada para a execução dos cálculos, principalmente, quando são empregados vários instrumentos de medição para a obtenção de superdeterminação de medidas. O ideal, em um processo de determinação de coordenadas espaciais de pontos de monitoramento, é obter um único sistema de referência, bem definido e estável para todos os elementos envolvidos no processo, evitando assim transformações de sistemas referenciais e cálculos desnecessários. Tal sistema de referência predefinido facilita a adaptação do método de verificação de instabilidade das bases, por meio da recessão espacial e, também, a calibração das coordenadas inicias do alvo de monitoramento.

Dessa forma, para a execução do subsistema de medição, primeiramente foi implantada a rede topográfica espacial, a qual impõe um sistema de referência absoluta local, para que, posteriormente, esta rede fosse utilizada como base de medição e de controle, para garantir maior estabilidade e oferecer maior confiabilidade interna no processo de monitoramento. Ainda, para minimizar os possíveis erros, foram considerados, como origem do sistema, os centros ópticos dos instrumentos de medição, evitando, assim, possíveis erros nas medições da altura dos instrumentos.

\subsubsection{Determinação da rede topográfica espacial}

Em geral, a solução utilizada para o cálculo e ajustamento de uma rede topográfica espacial é realizado separadamente em planimetria $(X, Y)$ e altimetria $(Z)$, ou seja, solução $(2 D+1 D)$. Para estes casos, são utilizadas as técnicas apresentadas no Capítulo 4. Entretanto, o ideal seria estabelecer uma relação que considera todos os elementos envolvidos no processo, ou seja, todas as observações $A z_{i P}^{O}, z_{i P}^{O}$ e $d_{i P}^{\prime O}$ e todas as incógnitas $\left(X_{P}, Y_{P}, Z_{P}\right)$, o que possibilitaria uma solução $(3 D)$, de forma direta. Assim, para preencher tal lacuna, foi desenvolvida pelo autor uma forma de realizar uma rede topográfica espacial com solução (3D). Tal rede tem sua parte teórica e modelagem matemática apresentada em detalhes no Capítulo 5 e, na sequência desta seção, sua parte prática.

A rede topográfica espacial de referência absoluta, para monitoramento, foi composta por 
seis bases, localizadas externamente ao alvo controlado pelo braço robótico, sendo estas denominadas por base 1, 2, 3, 4, 5 e 6, as quais estão dispostas na forma de um hexágono. A base 1, como mostra a Figura 8.2, teve como localização o terceiro andar do edifício do Instituto de Física de São Carlos (IFSC) e foi materializada por um tripé industrial de centragem forçada, uma caixa de comunicação diretamente ligada por cabos de rede ao switch e uma antena receptora dos sinais das estações totais robóticas, também diretamente ligada por cabos de rede ao switch. A Figura 8.2a mostra a localização da base 1, enquanto a Figura 8.2b mostra a sua materialização.

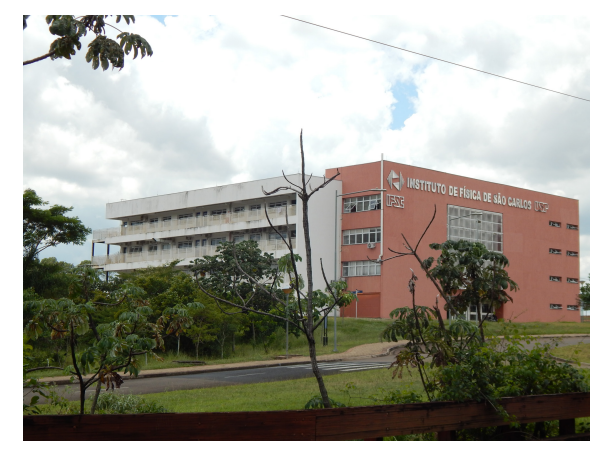

(a) Localização da base 1.

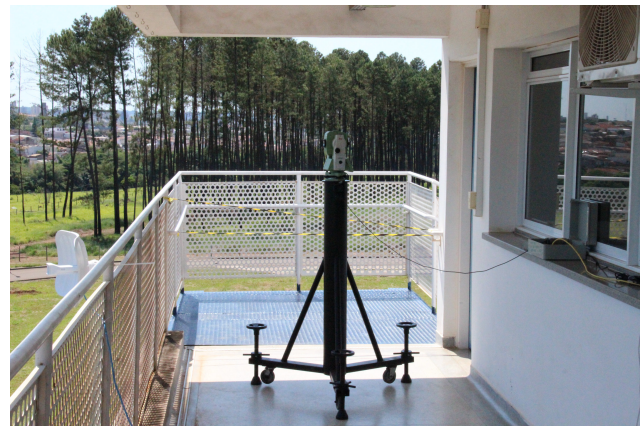

(b) Materialização da base 1.

Figura 8.2: Base 1 (IFSC).

A base 2, como mostra a Figura 8.3, localizada na marquise do edifício do Instituto de Química de São Carlos (IQSC), foi materializada também por um tripé industrial de centragem forçada, uma caixa de comunicação e uma antena emissora dos dados medidos. A Figura 8.3a mostra a localização da base 2, enquanto a Figura 8.3b mostra a sua materialização.

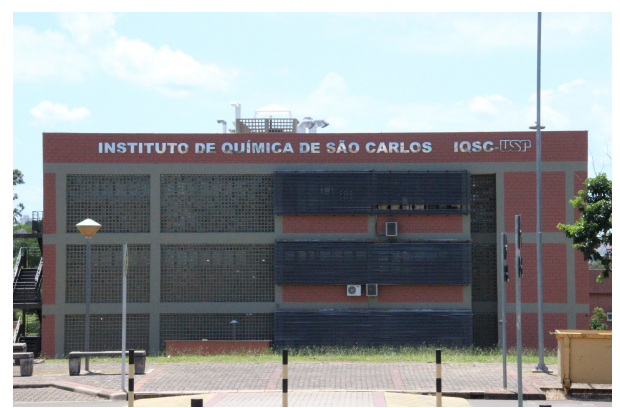

(a) Localização da base 2 .

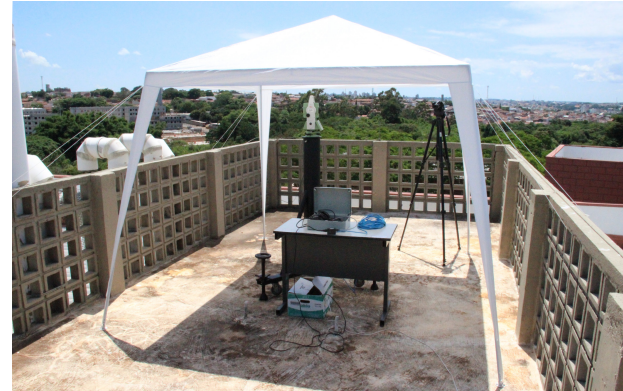

(b) Materialização da base 2.

Figura 8.3: Base 2 (IQSC).

Já, a base 6, vide Figura 8.4, foi engastada no perfil externo do terceiro andar do edifício Polo Terra e é composta por uma caixa de comunicação e uma antena emissora dos dados medidos. A Figura 8.4a mostra a localização da base 6, enquanto a Figura 8.4b mostra a sua materialização. Ressalta-se que, a instalação desta base foi realizada por meio de um guindaste e foi acompanhada pelos engenheiros e técnicos de segurança do trabalho do Campus 2 da USP São Carlos. 


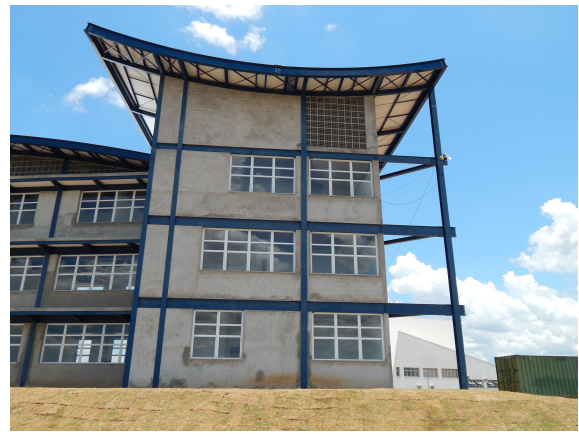

(a) Localização da base 6 .

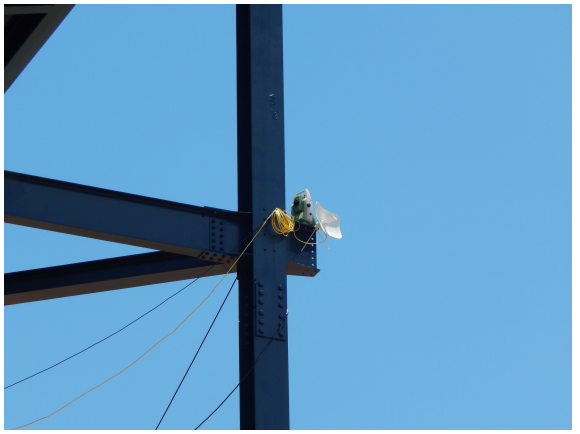

(b) Materialização da base 6.

Figura 8.4: Base 6 (Polo Terra).

Estas três bases foram utilizadas para efetuar as medições junto ao alvo em deslocamento, controlado pelo braço robótico.

A base 3, como pode ser visto na Figura 8.5a, teve seu alicerce triangular enterrado na rotatória da entrada Norte do Campus 2. A base 4, vide Figura 8.5b, teve seu alicerce triangular enterrado no gramado da portaria Norte do Campus 2. Já, a base 5, como mostra a Figura 8.5c, teve seu alicerce triangular enterrado no gramado na frente da central de segurança do Campus 2, ambas, compostas por base nivelante, carrier e prisma refletor.

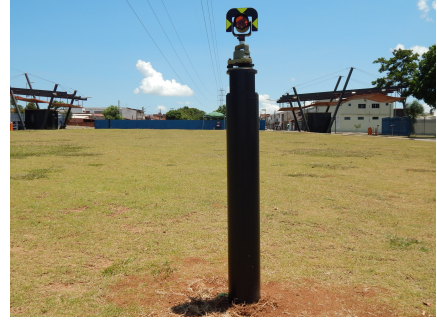

(a) Base 3

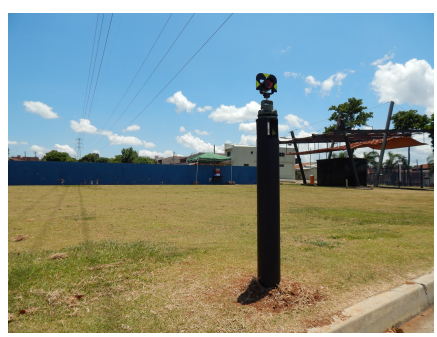

(b) Base 4 .

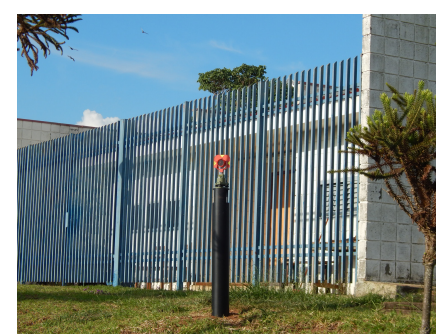

(c) Base 5.

Figura 8.5: Localização e materialização das bases 3, 4 e 5

Como pode ser visto, estas bases foram materializadas por um pilar de ferro estrutural e foram utilizadas para controle e verificação da estabilidade das bases 1,2 e 3, por meio do método de recessão espacial ou estação livre.

Após materializar essa rede no Campus 2 da USP São Carlos, adotou-se um procedimento, descrito a seguir, para realizar as medições, o ajustamento e o teste estatístico, com a finalidade de determinar as coordenadas espaciais de todas as bases envolvidas na rede absoluta. Assim, foi estabelecido um sistema espacial de referência local, com origem na base 1, conforme Tabela 8.1.

Tabela 8.1: Coordenadas espaciais arbitradas para a base 1.

\begin{tabular}{cccc}
\hline Ponto & $X[\boldsymbol{m}]$ & $Y[\boldsymbol{m}]$ & $Z[\boldsymbol{m}]$ \\
\hline $\mathbf{1}$ & $10.000,0000$ & $5.000,0000$ & 300,0000 \\
\hline
\end{tabular}


As etapas do procedimento adotado, para a realização da rede topográfica espacial, estão enumeradas na sequência:

1. Definiu-se a sequência de ocupação das bases, respectivamente como (1, 2, 3, 4, 5 e 6), em sentido anti-horário;

2. Adotou-se a estação total robótica TCRA1201+, escolhida aleatoriamente, dentre as estações totais robóticas utilizadas;

3. Fez-se a padronização do nivelamento de todas as bases da rede e, para isso, adotaram-se bases nivelantes e carrier, ambos de alta precisão;

4. Posicionou-se a estação total robótica na base (1), aguardaram-se quinze minutos para adaptação do instrumento ao ambiente e, em seguida, coletaram-se os dados metereológicos de temperatura e de pressão, para inseri-los no instrumento, o qual efetua a devida correção atmosférica. Ressalta-se que, em cada ocupação de base à vante, os dados metereológicos foram coletados novamente, para sua atualização e devido ajuste dos valores de correção;

5. Assim, iniciaram-se as observações da base (1) para a base (6), zerando o azimute da estação total robótica nesta última, considerada à ré;

6. Em seguida, realizaram-se vinte séries completas de leituras conjugadas (Face I) e (Face II) para cada medição angular e de distância inclinada para todas as bases da rede, utilizando o modo ATR. E, conforme o manual de referência LEICA (2007), em cada leitura conjugada, foi armazenado o valor médio (Face I\&II), cálculo efetuado internamente pela estação total robótica;

7. Ao encerrar as medições a partir da base (1), iniciou-se o mesmo procedimento para todas as bases da rede, em conformidade com o primeiro item;

8. Dessa forma, a estação total robótica ocupou a base (2) e visou, primeiramente, a base (1), para orientação à ré, porém, a partir de agora, com o uso da função Known BackSight Point, a qual calcula e orienta o azimute da estação total robótica automaticamente, para esta direção de orientação;

9. E, assim, de forma sucessiva foi efetuado o caminhamento até ocupar a base (6), na qual se encerrou o procedimento, com a orientação à ré na base (5) e as medições à vante em direção a cada base da rede topográfica espacial.

Após realizar esses procedimentos para todas as bases que compõem a rede, iniciou-se uma nova etapa, que corresponde à descarga e ao processamento dos dados coletados. Ao descarregar as observações no software LGO, pôde-se visualizar e conhecer os dados observados. E, para elevar o nível da precisão e da acurácia, como já foi mencionado, foram realizadas 20 séries de medições conjugadas para cada visada, e as medições que obtiveram um desvio em relação à média aritmética maior que 1" foram retiradas. A Tabela 8.2 mostra as medições já compensadas para cada linha de visada da rede topográfica espacial. 
Tabela 8.2: Dados observados para o cálculo da rede topográfica espacial.

\begin{tabular}{|c|c|c|c|c|c|c|c|c|}
\hline \multicolumn{2}{|c|}{ Pontos } & \multicolumn{3}{|c|}{$A z_{i P}^{O}$} & \multicolumn{3}{|c|}{$z_{i P}^{O}$} & \multirow{2}{*}{$\begin{array}{l}d_{i P}^{\prime O} \\
{[\boldsymbol{m}]}\end{array}$} \\
\hline Alfa [i] & Vante $[P]$ & $G\left[{ }^{\circ}\right]$ & $M[’]$ & $S["]$ & $G\left[{ }^{\circ}\right]$ & $M\left[{ }^{\prime}\right]$ & $S["]$ & \\
\hline 1 & 2 & 47 & 33 & 41,44 & 90 & 01 & 56,91 & 239,130 \\
\hline 1 & 3 & 38 & 09 & 43,75 & 90 & 24 & 09,87 & 831,416 \\
\hline 1 & 4 & 37 & 40 & 12,12 & 90 & 15 & 05,89 & 869,486 \\
\hline 1 & 5 & 33 & 18 & 41,88 & 90 & 03 & 49,21 & 924,319 \\
\hline 1 & 6 & 359 & 59 & 57,12 & 88 & 53 & 54,67 & 621,851 \\
\hline 2 & 3 & 34 & 24 & 30,71 & 90 & 32 & 45,82 & 596,782 \\
\hline 2 & 4 & 33 & 57 & 39,26 & 90 & 19 & 49,79 & 635,242 \\
\hline 2 & 5 & 28 & 27 & 05,84 & 90 & 04 & 18,37 & 695,044 \\
\hline 2 & 6 & 339 & 01 & 27,43 & 88 & 35 & 36,63 & 493,187 \\
\hline 2 & 1 & 227 & 33 & 44,28 & 89 & 58 & 16,00 & 239,130 \\
\hline 3 & 4 & 27 & 03 & 17,24 & 87 & 00 & 20,03 & 38,829 \\
\hline 3 & 5 & 357 & 04 & 0,13 & 87 & 40 & 31,84 & 119,007 \\
\hline 3 & 6 & 266 & 26 & 13,96 & 88 & 01 & 27,09 & 515,011 \\
\hline 3 & 1 & 218 & 09 & 35,32 & 89 & 36 & 21,13 & 831,415 \\
\hline 3 & 2 & 214 & 24 & 31,25 & 89 & 27 & 39,17 & 596,782 \\
\hline 4 & 5 & 344 & 16 & 06,28 & 88 & 10 & 00,98 & 87,543 \\
\hline 4 & 6 & 262 & 51 & 55,58 & 88 & 19 & 05,12 & 535,726 \\
\hline 4 & 1 & 217 & 40 & 05,96 & 89 & 45 & 26,64 & 869,485 \\
\hline 4 & 2 & 213 & 57 & 37,82 & 89 & 40 & 36,42 & 635,242 \\
\hline 4 & 3 & 207 & 03 & 19,62 & 92 & 59 & 45,17 & 38,829 \\
\hline 5 & 6 & 253 & 27 & 45,48 & 88 & 36 & 06,47 & 529,688 \\
\hline 5 & 1 & 213 & 18 & 37,64 & 89 & 56 & 45,10 & 924,319 \\
\hline 5 & 2 & 208 & 27 & 07,29 & 89 & 56 & 10,53 & 695,044 \\
\hline 5 & 3 & 177 & 03 & 54,54 & 92 & 19 & 40,74 & 119,007 \\
\hline 5 & 4 & 164 & 16 & 07,61 & 91 & 50 & 08,41 & 87,543 \\
\hline 6 & 1 & 179 & 59 & 53,51 & 91 & 06 & 35,34 & 621,852 \\
\hline 6 & 2 & 159 & 01 & 34,87 & 91 & 24 & 43,86 & 493,188 \\
\hline 6 & 3 & 86 & 26 & 11,29 & 91 & 58 & 55,09 & 515,012 \\
\hline 6 & 4 & 82 & 51 & 47,09 & 91 & 41 & 17,78 & 535,726 \\
\hline 6 & 5 & 73 & 27 & 43,85 & 91 & 24 & 13,86 & 529,688 \\
\hline
\end{tabular}

As coordenadas aproximadas das bases (2, 3, 4, 5 e 6), ilustradas pela Tabela 8.3, foram calculadas por meio da rotina computacional elaborada no software Python, considerando as modelagens matemáticas apresentadas na seção teórica, especificamente no Capítulo 5, que dá embasamento à elaboração, à execução e ao processamento da rede topográfica espacial. 
Tabela 8.3: Coordenadas aproximadas das bases.

\begin{tabular}{cccc}
\hline Pontos & $X[\boldsymbol{m}]$ & $Y[\boldsymbol{m}]$ & $Z[\boldsymbol{m}]$ \\
\hline $\mathbf{1}$ & $10.000,0000$ & $5.000,0000$ & 300,0000 \\
2 & $10.176,4756$ & $5.161,3633$ & 299,8000 \\
$\mathbf{3}$ & $10.513,7003$ & $5.653,7072$ & 294,1598 \\
$\mathbf{4}$ & $10.531,3373$ & $5.688,2410$ & 296,1870 \\
$\mathbf{5}$ & $10.507,6146$ & $5.772,4576$ & 298,9859 \\
$\mathbf{6}$ & $9.999,9874$ & $5.621,7296$ & 311,9433 \\
\hline
\end{tabular}

Consequentemente, a mesma rotina computacional gerou as matrizes abaixo para o ajustamento das coordenadas espaciais, suas respectivas precisões e, posteriormente, a verificação do grau de confiabilidade da realização da rede topográfica espacial.

$\left.\mathrm{V}=\left[\begin{array}{c}8,31 \cdot 10^{-6} \\ -3,69 \cdot 10^{-4} \\ -2,21 \cdot 10^{-4} \\ -3,00 \cdot 10^{-4} \\ -9,62 \cdot 10^{-4} \\ -5,42 \cdot 10^{-4} \\ 6,44 \cdot 10^{-5} \\ -1,98 \cdot 10^{-4} \\ 5,74 \cdot 10^{-4} \\ -3,17 \cdot 10^{-4} \\ \vdots \\ 1,55 \cdot 10^{-5} \\ 1,44 \cdot 10^{-5} \\ 1,45 \cdot 10^{-5} \\ 5,35 \cdot 10^{-5} \\ 2,50 \cdot 10^{-6} \\ 2,28 \cdot 10^{-5} \\ -4,39 \cdot 10^{-6} \\ 1,14 \cdot 10^{-5} \\ 2,55 \cdot 10^{-5} \\ -7,71 \cdot 10^{-7}\end{array}\right] \quad A=\left[\begin{array}{ccccc}7,38 \cdot 10^{-1} \\ 0 & 6.75 \cdot 10^{-1} & -8,36 \cdot 10^{-4} & \ldots & 0 \\ 0 & 0 & 0 & \cdots & 0 \\ 0 & 0 & 0 & \cdots & 0 \\ 0 & 0 & 0 & \cdots & 0 \\ \vdots & \vdots & \vdots & \ddots & \vdots \\ 0 & 0 & 0 & \cdots & 0 \\ 1,89 \cdot 10^{-3} & 7,26 \cdot 10^{-4} & 0 & \cdots & 0 \\ 0 & 0 & 0 & \cdots & 0 \\ 0 & 0 & 0 & \cdots & 0 \\ 0 & 0 & 0 & \cdots & 0\end{array}\right] \quad X=\left[\begin{array}{c}2,61 \cdot 10^{-3} \\ -2,03 \cdot 10^{-3} \\ -2,24 \cdot 10^{-3} \\ 8,53 \cdot 10^{-4} \\ 6,86 \cdot 10^{-3} \\ -4,88 \cdot 10^{-3} \\ -5,68 \cdot 10^{-3} \\ -2,55 \cdot 10^{-3} \\ 6,57 \cdot 10^{-4} \\ 2,94 \cdot 10^{-3} \\ \vdots \\ \\ 3,29 \cdot 10^{-3} \\ 2,77 \cdot 10^{-1} \\ 1,76 \cdot 10^{-3} \\ 3,61 \cdot 10^{-3} \\ 9,00 \cdot 10^{-1} \\ 7,52 \cdot 10^{-4} \\ 1,46 \cdot 10^{-3} \\ 9,29 \cdot 10^{-1} \\ -1,09 \cdot 10^{-3} \\ 2,69 \cdot 10^{-3} \\ 9,96 \cdot 10^{-1} \\ -8,07 \cdot 10^{-3} \\ -4,66 \cdot 10^{-3} \\ 5,49 \cdot 10^{-1}\end{array}\right] \quad \begin{array}{c} \\ -2,72 \cdot 10^{-6} \\ -5,18 \cdot 10^{-6} \\ -2,01 \cdot 10^{-5} \\ 1,03 \cdot 10^{-5} \\ -1,11 \cdot 10^{-5} \\ 2,47 \cdot 10^{-5} \\ -2,76 \cdot 10^{-5} \\ -3,62 \cdot 10^{-5} \\ -4,67 \cdot 10^{-6}\end{array}\right]$

A Tabela 8.4 mostra as coordenadas espaciais processadas e ajustadas, seguidas de suas respectivas precisões.

Tabela 8.4: Coordenadas e precisões ajustadas das bases.

\begin{tabular}{cccccccc}
\hline Pontos & $X[\mathbf{m}]$ & $\sigma_{X}[\mathbf{m m}]$ & $Y[\boldsymbol{m}]$ & $\sigma_{Y}[\mathbf{m m}]$ & $Z[\mathbf{m}]$ & $\sigma_{Z}[\mathbf{m m}]$ & $\sigma_{X Y Z}[\mathbf{m m}]$ \\
\hline $\mathbf{2}$ & $10.176,4755$ & 0,5433 & $5.161,3676$ & 0,4911 & 300,0767 & 0,9450 & 1,1956 \\
$\mathbf{3}$ & $10.513,7021$ & 1,5747 & $5.653,7108$ & 1,2542 & 295,0598 & 2,0365 & 2,8636 \\
$\mathbf{4}$ & $10.531,3381$ & 1,6496 & $5.688,2424$ & 1,2911 & 297,1165 & 2,0933 & 2,9614 \\
$\mathbf{5}$ & $10.507,6135$ & 1,8805 & $5.772,4603$ & 1,2436 & 299,9823 & 2,1514 & 3,1163 \\
$\mathbf{6}$ & $9.999,9793$ & 1,8710 & $5.621,7250$ & 0,7666 & 312,4928 & 2,0555 & 2,8834 \\
\hline
\end{tabular}

Com o intuito de detectar inconsistências do ajustamento, de verificar prováveis causas da falha da suposição básica e de analisar as diferenças encontradas nas coordenadas ajustadas da rede, realizou-se uma análise, aplicando o Teste Bilateral do Qui-Quadrado $\chi^{2}$, com nível 
de significância $\alpha=0,001 \%$, com variância "a priori" $\sigma_{0}^{2}=0,001$ e com variância "a posteriori" $\hat{\sigma}_{0}^{2}$ calculada, conforme ilustra a Tabela 8.5.

Tabela 8.5: Teste do $\chi^{2}$ para verificação e análise da confiabilidade da rede espacial.

\begin{tabular}{cccccc}
\hline Rede & $\hat{\sigma}_{0}^{2}$ & $\chi_{g l, \frac{\alpha}{2}}^{2}$ & $\chi_{g l, 1-\frac{\alpha}{2}}^{2}$ & $\chi^{* 2}$ & Hipótese \\
\hline Espacial & 0,0005 & 32,6495 & 141,8659 & 35,8150 & Aceito \\
\hline
\end{tabular}

Sendo,

$\chi^{* 2}$ - Valores calculados para o teste do qui-quadrado $\chi^{2}$;

$\chi_{g l, \frac{\alpha}{2}}^{2}$ e $\chi_{g l, 1-\frac{\alpha}{2}}^{2}$ - Valores obtidos da tabela de distribuição do qui-quadrado $\chi^{2}$;

$\alpha$ - Nível de significância multidimensional;

$g l$ - Grau de liberdade, obtido pela diferença entre o número de equações $n$ e o número de incógnitas $u$ a ser determinado, sendo: $g l=90-15=75$, para a rede espacial.

Os valores calculados para $\chi^{* 2}$, para a rede topográfica espacial, encontraram-se entre aqueles tabelados $\chi_{g l, \frac{\alpha}{2}}^{2}$ e $\chi_{g l, 1-\frac{\alpha}{2}}^{2}$, ou seja, $\chi_{g l, \frac{\alpha}{2}}^{2}<\chi^{* 2}>\chi_{g l, 1-\frac{\alpha}{2}}^{2}$. Dessa maneira, pode-se assumir que a hipótese básica foi aceita e o ajustamento mostrou-se consistente e bem condicionado ao nível de confiança de $99,999 \%$.

Por fim, a Figura 8.6 mostra a visualização gráfica da rede topográfica espacial e suas respectivas elipses de erros.

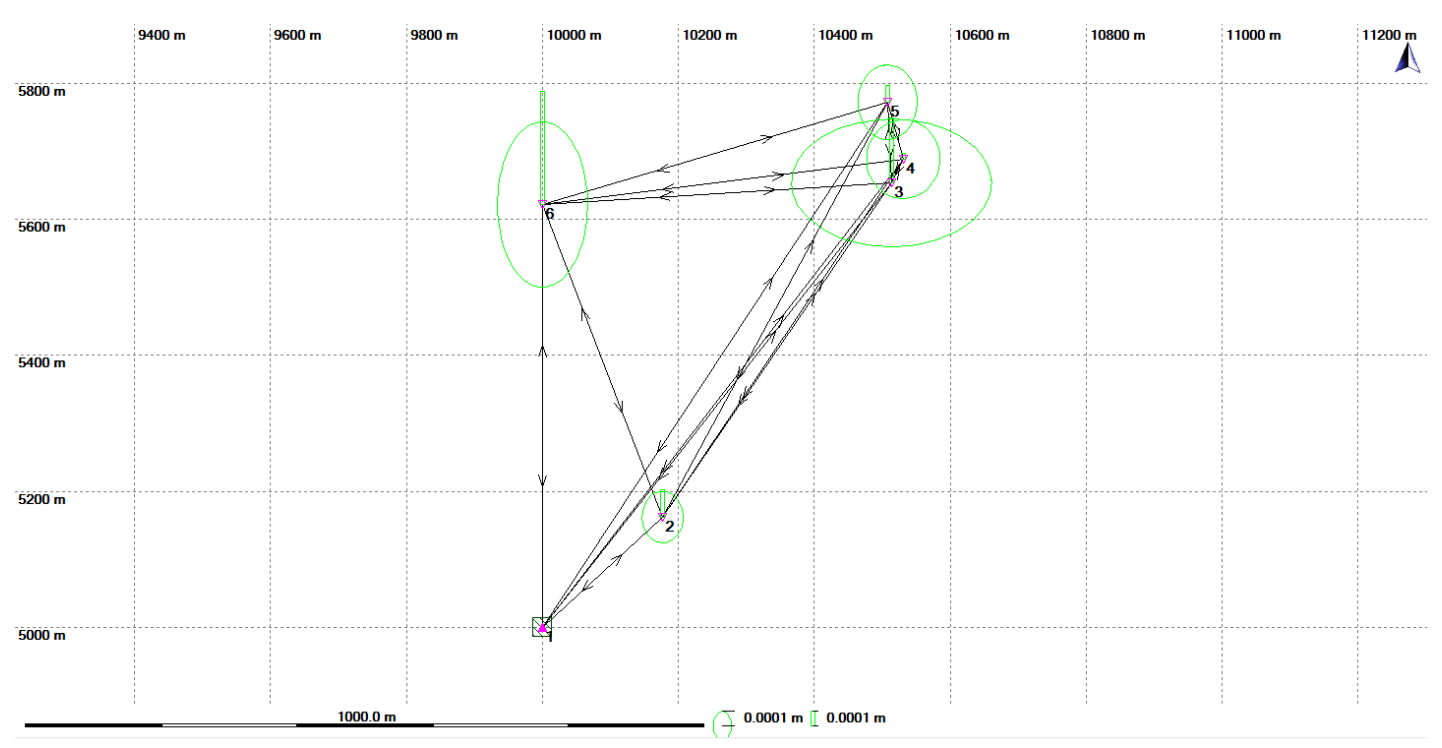

Figura 8.6: Rede topográfica espacial e elipse dos erros.

Dessa forma, a rede topográfica espacial foi implantada para servir como base e referência absoluta para todo o processo de monitoramento. 


\subsubsection{Determinação das coordenadas espaciais do ponto de monitoramento}

Com a realização da rede topográfica espacial, foi obtido um sistema de referencial absoluto para o processo de monitoramento. O layout apresentado pela Figura 8.7 ilustra a rede composta pelos elementos de medição, que são as bases com estações totais robóticas e pelos elementos de controle, que são as bases com prismas refletores.

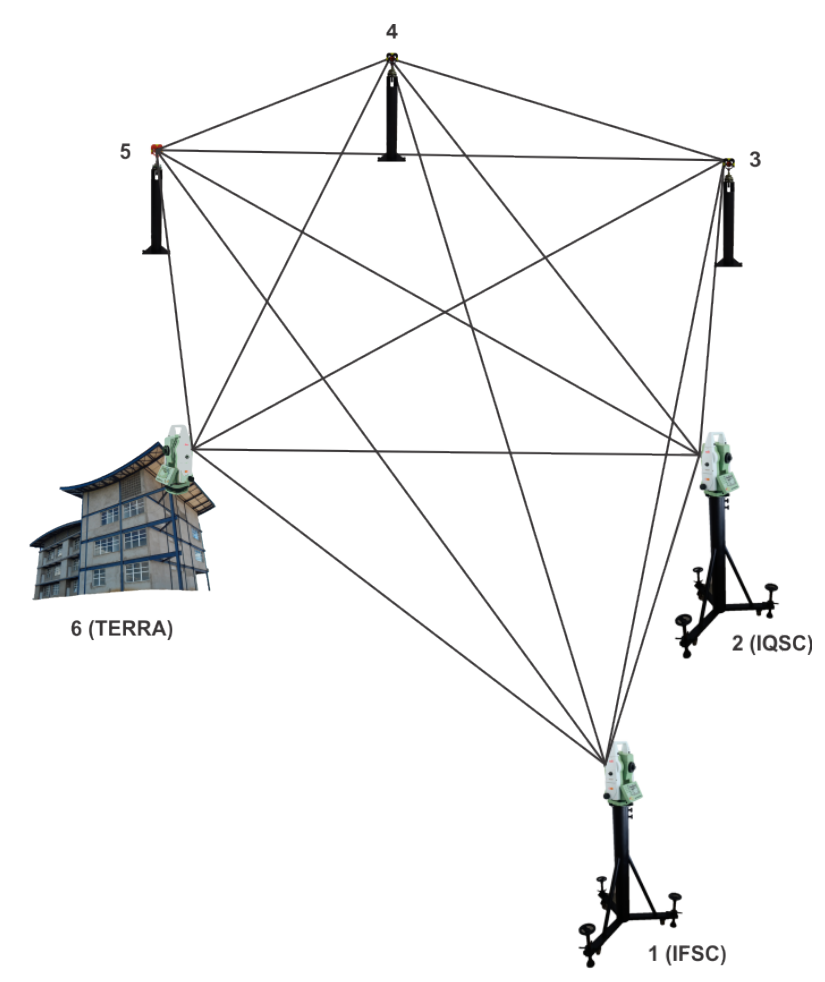

Figura 8.7: Rede topográfica espacial de referência absoluta e controle.

Ainda, no processo de monitoramento, foi definida a localização do alvo em deslocamento, controlado pelo braço robótico. Este robô foi engastado em uma estrutura de concreto armado, localizada na entrada Norte do Campus 2 e, com o objetivo de preservar todos os equipamentos, foi montada uma tenda provisória, como mostra a Figura 8.8. Na Figura 8.8a, mostra-se a operação do robô durante o dia e, na Figura 8.8 b mostra-se a operação do robô durante a noite, inclusive no instante em que a TCRP1201+ realiza uma de suas medições.

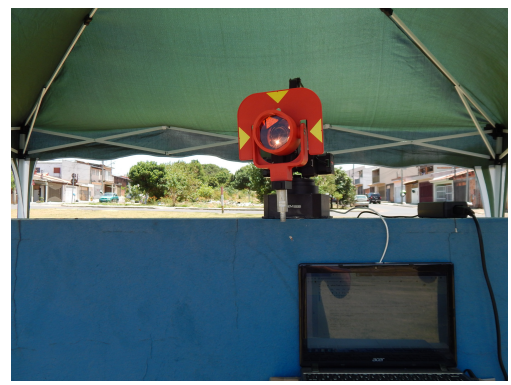

(a) Operação do robô de dia.

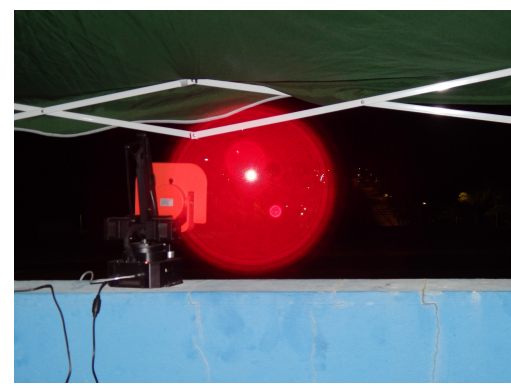

(b) Operação do robô a noite.

Figura 8.8: Localização e operação do robô. 
Devido ao fato de o braço robótico ter o seu próprio sistema de coordenada espacial, foi necessário estabelecer uma posição inicial para calibração e integração do mesmo ao sistema de coordenadas espaciais, determinadas pela rede topográfica espacial. Dessa forma, foi realizado um processo de aprendizagem de máquina em que todas as coordenadas do sistema do braço robótico, fossem convertidas para o sistema de coordenadas local predefinido pela rede topográfica espacial, a qual é a referência absoluta do sistema de monitoramento.

A movimentação deste robô ocorreu por meio de uma rotina computacional, realizada na plataforma Python, para executar as tarefas predefinidas, bem como a movimentação espacial do prisma refletor, de forma contínua, e em ciclos de cinco minutos. Ressalta-se que as coordenadas espaciais de movimentação do braço robótico foram inseridas de forma aleatória. Para isso, foi definido um limite de atuação para o braço robótico, respeitando todas as características operacionais, apresentadas na Figura 3.11. Dessa forma, deu-se início ao processo de monitoramento, com medições realizadas continuamente, em um período de cinco em cinco minutos para todos os elementos ativos do sistema, que são as estações totais robóticas e o braço robótico $u$ Arm Swift Pro.

A Figura 8.9 ilustra a sequência lógica do monitoramento aplicado neste trabalho. Primeiramente, as estações totais robóticas, ao iniciarem seus ciclos, realizaram medições relativas aos três pontos de controle ( 3,4 e 5), para a recessão espacial e para a verificação da estabilidade das bases de medição. Logo em seguida, realizaram a medição relativa ao ponto de monitoramento $P$, para a determinação das coordenadas espaciais. Esse ciclo se repetiu com a periodicidade predefinida, para que, assim, fossem obtidas as variações das coordenadas espaciais do alvo $P$ em deslocamento controlado. Com isso, calcularam-se as variações das coordenadas espaciais obtidas por cada método considerado nesta tese. E, dessa forma, foi possível confrontar os resultados e realizar análises comparativas. 


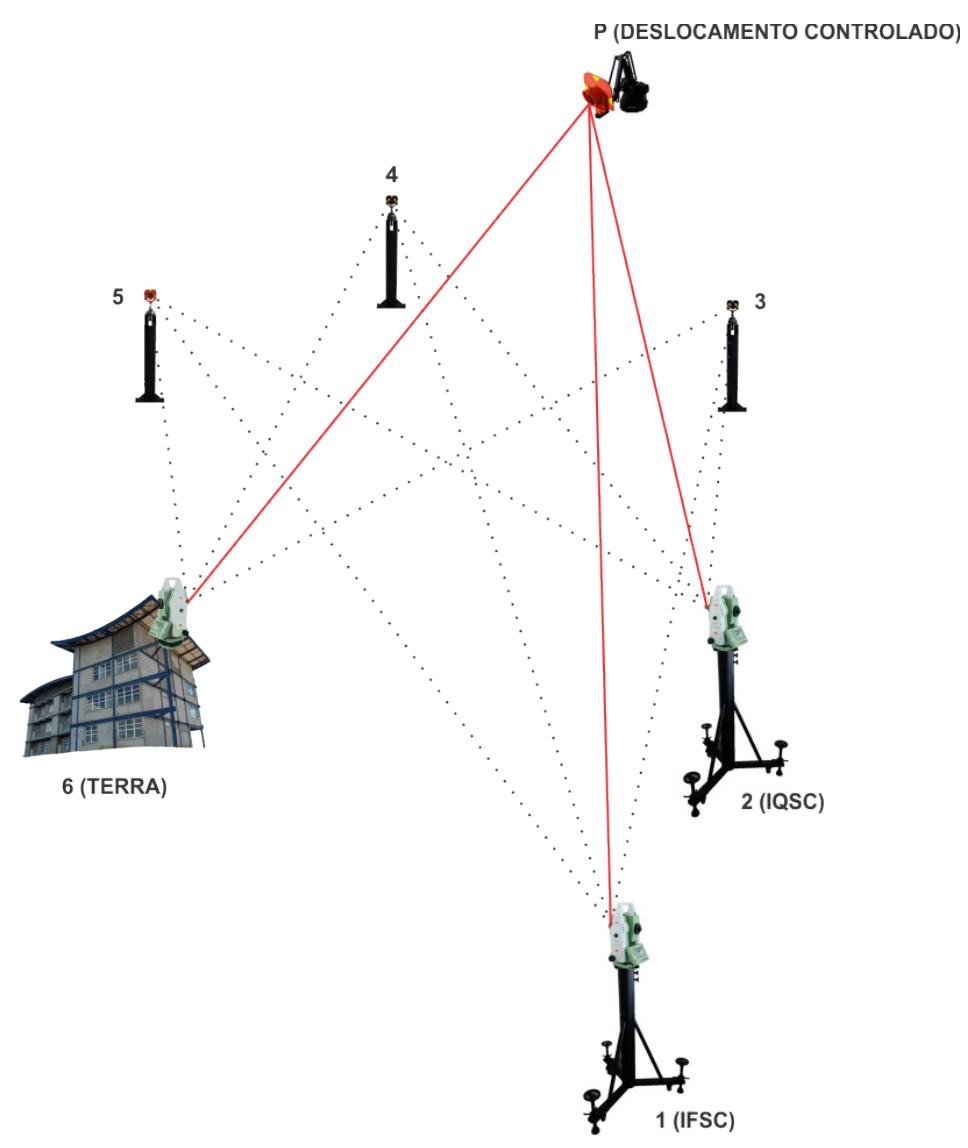

Figura 8.9: Esquema da sequência lógica do processo de monitoramento.

Para a execução do processo de monitoramento contínuo, periódico e em tempo real, foram desenvolvidos três sistemas integrados para cada ambiente de medição. Tais sistemas foram desenvolvidos de forma que todos os componentes operassem de forma integrada. A Figura 8.10 mostra os elementos de integração de todos os ambientes de medição.

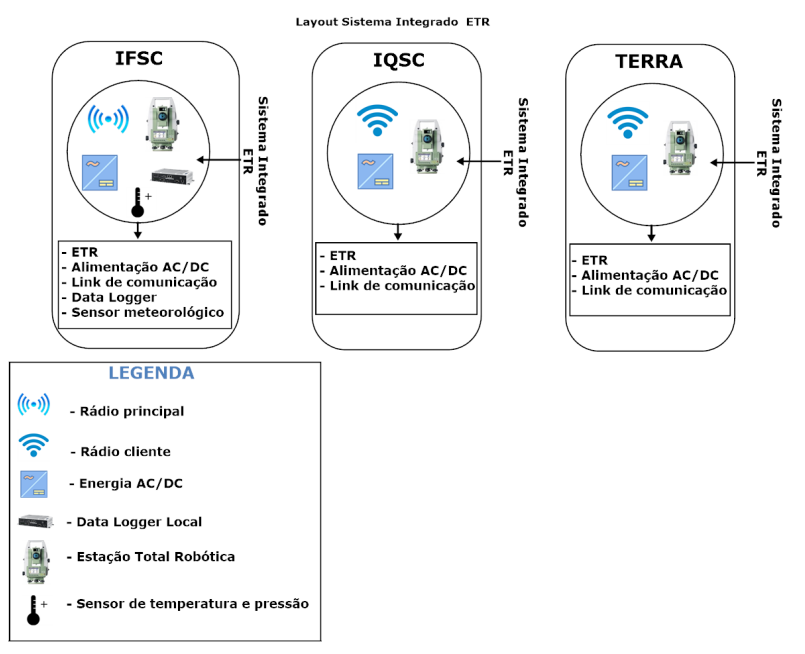

Figura 8.10: Layout do sistema integrado, com três estações totais robóticas.

Ressalta-se que este sistema integrado relacionou os elementos envolvidos e, assim, agrupou o subsistema de medição ao subsistema de comunicação, como pode ser visto na próxima seção. 


\subsection{Subsistema de comunicação}

Para que estes ambientes de medição com sistema integrado operassem e se comunicassem sincronizadamente, foi desenvolvido um sistema de comunicação, em tempo real, entre todos os ambientes. A Figura 8.11 mostra detalhadamente o diagrama do sistema de comunicação entre os ambientes de medição e o CORE do sistema de monitoramento.

O ambiente Polo Terra foi composto pela TCA1201+ para medição, por um conversor de mídia para conversão dos sinais dos dados medidos e conexão intranet, por uma antena de rádio cliente para emissão do dados e por uma fonte de energia para alimentação. O ambiente IQSC foi composto pela TCRA1201+ para medição e, basicamente, pelos mesmos equipamentos do ambiente Polo Terra. Já o ambiente IFSC, foi composto pela TCRP1201+ para medição, por um sensor metereológico para leitura de temperatura e pressão, por um conversor de mídia e por uma fonte de energia.

Portanto, o que diferencia o ambiente IFSC dos demais, é que nele também se localiza o CORE do sistema de monitoramento, composto por uma antena de rádio principal para recepção de dados dos outros ambientes, por um switch de rede para transmissão direta de dados ao datalogger, que é o software de gerenciamento GeoMos, para efetuar a gestão contínua e, em tempo real, do sistema de monitoramento.

Notar que todos os ambientes de medição foram protegidos por nobreak, isso para estabilizar oscilações de energia externa e manter o sistema operante o maior tempo possível, em caso de queda de energia.

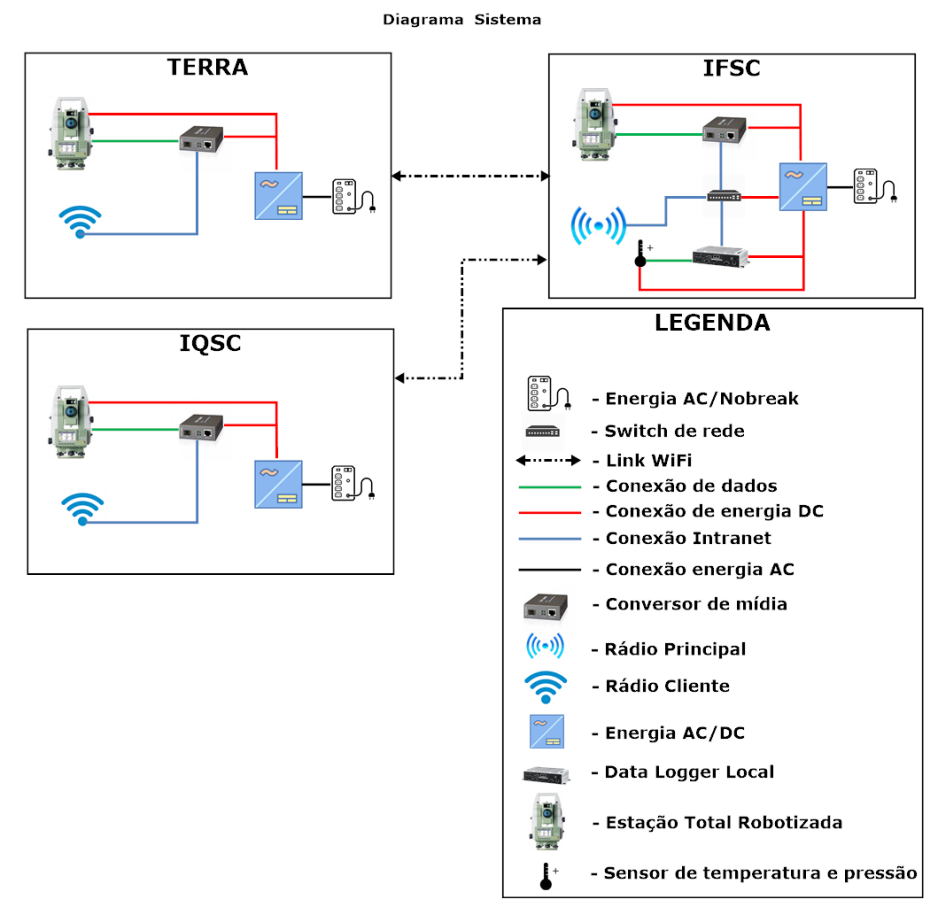

Figura 8.11: Diagrama do sistema operacional de cada ambiente. 
Dessa forma, para melhor visualização da comunicação entre os ambientes de medição e o CORE do sistema de monitoramento, apresenta-se o esquema de comunicação na Figura 8.12. O link de comunicação de rádio entre as estações totais robóticas foi estabelecido com duas antenas de rádio cliente e uma antena de rádio principal.

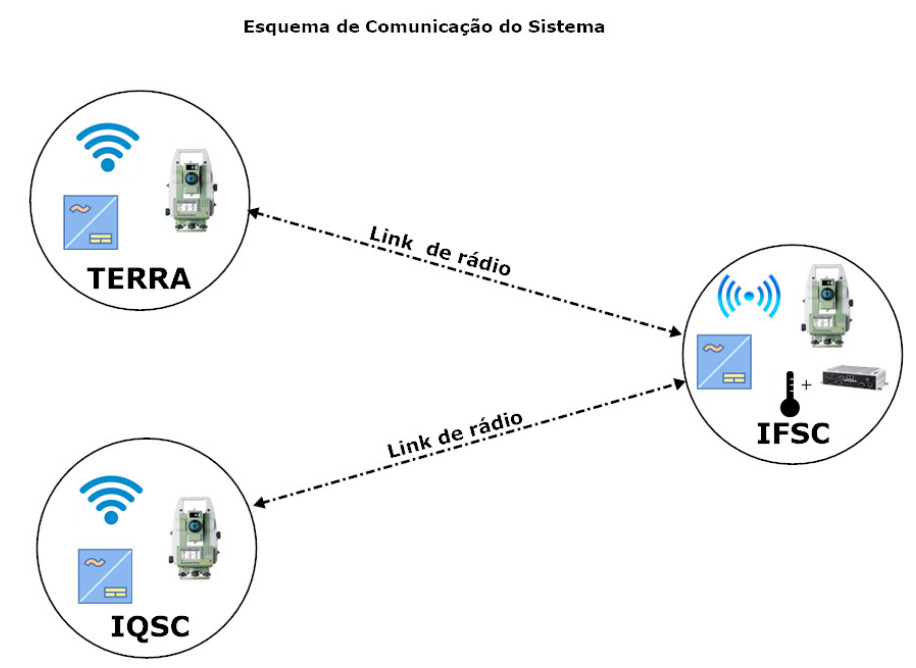

Figura 8.12: Esquema de comunicação do sistema.

Para ilustrar a dimensão e abrangência do subsistema de comunicação, a Figura 8.13 mostra a espacialização dos elementos do sistema de monitoramento. Trata-se do Layout da comunicação via link de rádio em tempo real.

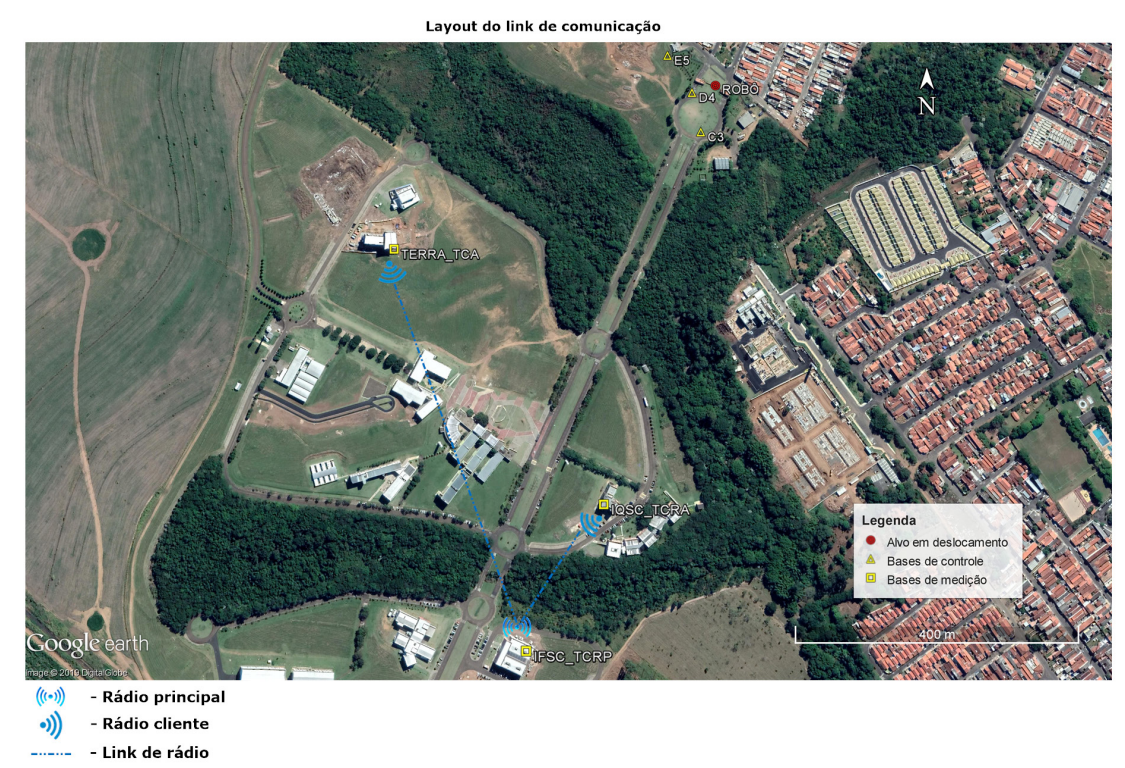

Figura 8.13: Layout do link de comunicação.

Como pode ser visto, as estações do Polo Terra e IQSC enviaram dados para a central, localizada no IFSC, que os recebeu pela antena principal. E a antena principal enviou todos os dados para o CORE do sistema que, automaticamente, alimentou o banco de dados e sucessivamente o disponibilizou para o subsistema de processamento e alarme. 


\subsection{Subsistema de processamento e alarme}

De posse do Banco de Dados (BD) gerado pelo software GeoMos, foi possível acessar e extrair todas as informações pertinentes às observações. Por se tratar de um banco de dados complexo, foi desenvolvido um subsistema de processamento, para que todos os modelos matemáticos de determinações de coordenadas espaciais considerados fossem confrontados.

Este subsistema de processamento, desenvolvido pelo autor, contém uma organização computacional que corresponde a uma estrutura de armazenamento, baseada em camadas de atributos distintos. Cada camada dessa estrutura de armazenamento apresenta-se sistematizada, como uma tabela duplamente indexada por atributos bases, em relação aos demais dados brutos. A utilização dessa organização se assemelha aos conceitos de indexação por chave primária, utilizados em sistemas de banco de dados, que promovem uma identificação única para cada elemento do sistema (ELMASRI; NAVATHE, 2016).

O armazenamento das camadas de atributos, organizadas em razão dos índices de controle definidos pelo usuário, é realizado por meio de um vetor unidimensional de camadas. Cada célula do vetor é responsável por arquivar uma única camada de elementos da estrutura, deste modo o vetor deve possuir, inicialmente, o mesmo número de células e de atributos presentes na tabela de dados brutos.

Em vista dessa organização, o acesso a um dado armazenado na estrutura proposta deve ser realizado por meio da posição da camada, no vetor de camadas, e dos valores do índice de controle. Esse acesso pode ser efetuado tanto para um valor único quanto para a recuperação de todas as informações presentes na camada. De posse dessa estrutura preenchida com os dados reorganizados, foi possível realizar atividades de manipulação dos dados com o objetivo de realizar cálculos, provenientes do monitoramento.

A Figura 8.14 ilustra, de maneira genérica, o sistema de gerenciamento proposto. O eixo $Z$ representa o vetor de camadas, responsável pelo controle de acesso a cada conjunto de atributos. Os eixos $X$ e $Y$ representam o sistema de referenciamento, dentro das camadas de informações, responsáveis por possibilitar o acesso específico a uma informação.

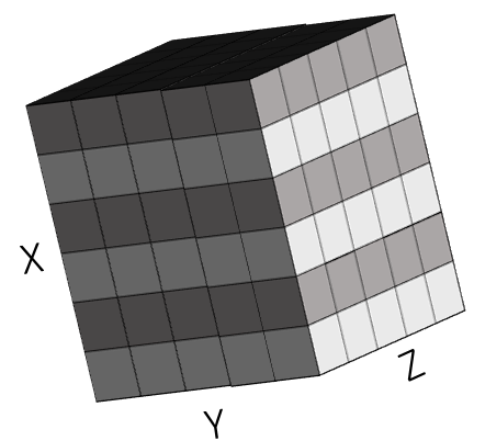

Figura 8.14: Concepção do sistema de gerenciamento proposto. 
A estruturação do sistema de gerenciamento de dados, proposto neste trabalho, pode ser dividida em 3 etapas, conforme mostra a Figura 8.15.

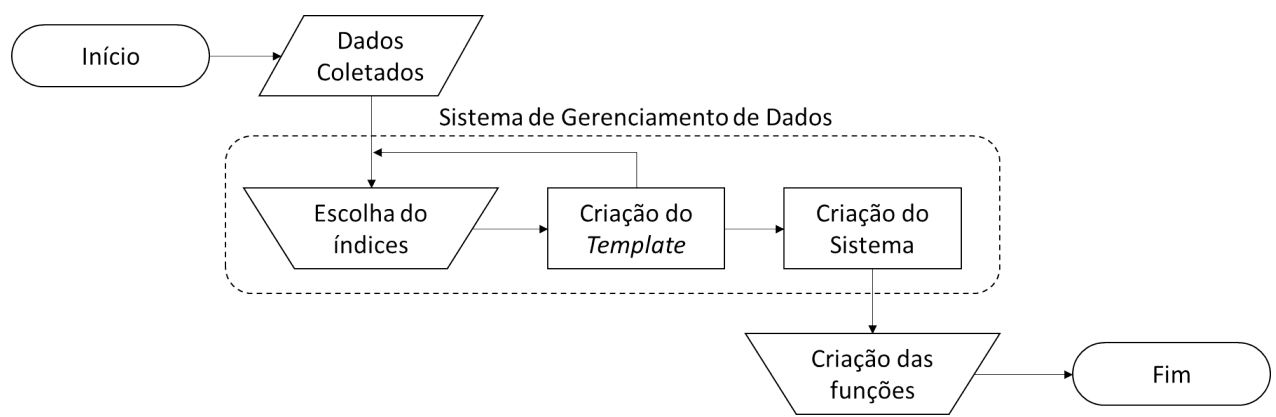

Figura 8.15: Etapas do sistema de gerenciamento de dados.

De posse dos dados coletados, realizou-se a importação dos mesmos, o qual pode ser realizado de modo manual ou automático, para o ambiente de desenvolvimento. Em seguida, após a importação dos dados brutos para o sistema, iniciou-se a Etapa 1 da estruturação dos dados brutos, para o sistema de gerenciamento proposto.

$\mathrm{Na}$ Etapa 1, foram definidos os atributos que formam o sistema de indexação das camadas de atributos. Por meio da análise da tabela de dados brutos, coletados e organizados anteriormente, foram escolhidos dois atributos, presentes nas colunas de elementos da tabela, que possibilitaram, de maneira única, o acesso a cada elemento dos demais atributos. Esta definição representa uma etapa essencial, uma vez que todo o sistema de gerenciamento de dados será estruturado a partir da sua escolha.

A Etapa 2 consiste na validação, de maneira automática, das atividades do passo anterior, por meio da verificação dos possíveis valores dos atributos selecionados e de sua capacidade de acessar todos os elementos da tabela bruta. Com base nessa validação, o sistema possui uma condição em caso de falha, em que obrigatoriamente deve retroceder à Etapa 1 para a escolha de novos atributos de controle. Em caso de êxito da validação, o sistema deve estabelecer, em razão dos atributos selecionados, uma tabela duplamente indexada, que será utilizada como template para as camadas a serem criadas. Cada um dos atributos selecionados, necessariamente, deve ser responsável pelo controle de uma dimensão da tabela. 
A Figura 8.16 ilustra, de maneira simplificada, a elaboração do template com os índices de controle e especificamente na Figura 8.16a, apresenta-se uma tabela de dados brutos. Posteriormente, a Figura 8.16b expõe uma tabela em que os atributos from e to, da tabela anterior, foram selecionados como índices de controle para o template desse sistema.

\begin{tabular}{|c|c|c|c|c|}
\hline from & to & $\beta$ [rad] & $\ldots$ & $A z$ [rad] \\
\hline 1 & a & & $\ldots$ & \\
\hline 2 & b & & $\ldots$ & \\
\hline 3 & c & & $\ldots$ & \\
\hline 4 & d & & $\ldots$ & \\
\hline 5 & e & & $\ldots$ & \\
\hline 6 & f & & $\ldots$ & \\
\hline
\end{tabular}

(a) Dados brutos.

\begin{tabular}{|c|c|c|c|c|c|c|}
\hline & a & b & c & d & e & f \\
\hline 1 & & & & & & \\
\hline 2 & & & & & & \\
\hline 3 & & & & & & \\
\hline 4 & & & & & & \\
\hline 5 & & & & & & \\
\hline 6 & & & & & & \\
\hline
\end{tabular}

(b) Índices de controle do template.

Figura 8.16: Criação do template do sistema.

A Etapa 3 do procedimento consiste na reestruturação do conjunto de dados brutos. Com o template de armazenamento dos dados criados, o sistema deve gerar instâncias dessa estrutura e preenchê-las com os elementos da tabela de dados brutos. Cada atributo deve apenas ocupar uma única instância de template e o preenchimento necessariamente deve obedecer aos valores dos índices de controle da camada. Com o término do preenchimento de cada instância, é efetuada a adição dessa estrutura no vetor de controle das camadas de informação. Na sequência, o sistema obrigatoriamente deve produzir uma lista com o posicionamento de cada estrutura no vetor de camadas, mapping, de maneira a auxiliar as futuras manipulações dos dados, em etapas de programação de cálculos. Ao término das 3 etapas de reorganização dos dados brutos, a estrutura básica do sistema de gerenciamento de dados ficou completa. A partir desse ponto, tornou-se possível o acesso, de maneira eficiente de cada elemento dos atributos e, assim, foram desenvolvidas as aplicações, conforme as necessidades.

Com o levantamento de dados do monitoramento, realizou-se a estruturação dos dados, conforme o sistema de gerenciamento apresentado. Neste estágio, definiu-se a chave primária indexadora do sistema de gerenciamento. A escolha dos atributos para a chave indexadora foi determinada em razão dos pontos ocupados pelas estações totais robóticas e do ponto visado monitorado, respectivamente, que apresentavam valores numéricos referentes à identificação de cada coleta. Após a definição da chave, foram implementadas funções de manipulação de dados. Neste caso, realizou-se o cálculo de deslocamento por meio dos métodos considerados.

Para verificar a consistência do sistema proposto, os resultados estão apresentados em função da aplicação testada. A análise dos resultados é abordada em três etapas: (1) input dos dados brutos no sistema, (2) gerenciamento de dados do sistema e (3) output de dados processados no sistema. A tabulação escolhida para a saída de dados das estações totais robóticas são apresentadas na Figura 8.17. 


\begin{tabular}{|c|c|c|c|c|c|c|}
\hline ID & from & to & $\ldots$ & $\boldsymbol{d}^{\prime}[\boldsymbol{m}]$ & $\boldsymbol{\beta}$ [rad] & $\boldsymbol{A z}$ [rad] \\
\hline $\mathbf{1}$ & 1 & 4 & $\ldots$ & 556,6852 & $-0,0256522$ & 1,4356858 \\
\hline $\mathbf{2}$ & 4 & 5 & $\ldots$ & 652,8965 & $-0,0038633$ & 0,6152125 \\
\hline $\mathbf{3}$ & 2 & 3 & $\ldots$ & 887,7788 & $-0,0028962$ & 0,6701225 \\
\hline$:$ & $:$ & $:$ & $:$ & $:$ & $:$ & $:$ \\
\hline $\mathbf{n}-\mathbf{1}$ & 1 & 8 & $\ldots$ & 556,6528 & $-0,0256522$ & 1,4356896 \\
\hline $\mathbf{n}$ & 2 & 7 & $\ldots$ & 652,8988 & $-0,0038633$ & 0,6152526 \\
\hline
\end{tabular}

Figura 8.17: Input dos dados brutos no sistema.

Como se pode verificar, os dados do levantamento são, basicamente, os identificadores dos pontos, que correspondem aos valores de medidas de distância, de direções e de ângulos verticais para cada ponto de observação.

A Figura 8.18 representa o sistema de gerenciamento, o qual compreende acesso, armazenamento e manipulação de dados. A estrutura de armazenamento é constituída por meio de um vetor unidimensional de matrizes, nas quais as linhas e as colunas constituem a chave indexadora. Cada elemento do vetor corresponde a um atributo do conjunto de dados brutos. A dimensão das matrizes é definida em função do universo de medições e pode ser compreendida como camadas.

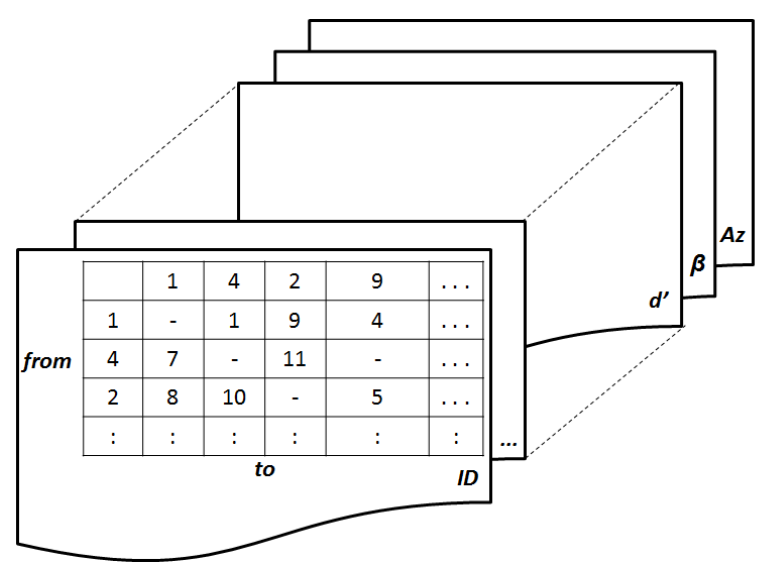

Figura 8.18: Gerenciamento dos dados do sistema.

A estrutura de acesso é baseada em três características do sistema: a primeira corresponde à posição dos elementos no vetor de matrizes, em que cada posição se relaciona aos atributos do conjunto de dados brutos, que se equivale ao mapping, descrito na Figura 8.19; a segunda e a terceira relacionam-se à chave indexadora e funcionam como acesso aos registros de cada atributo. A manipulação dos dados foi realizada por meio dessas três características descritas.

\begin{tabular}{|c|c|c|c|c|c|}
\hline Atributo & $I D$ & $\ldots$ & $\boldsymbol{d}^{\prime}[\mathrm{m}]$ & $\boldsymbol{\beta}[\mathrm{rad}]$ & $\boldsymbol{A z}[\mathrm{rad}]$ \\
\hline Posição & 0 & $\ldots$ & $\mathrm{n}-2$ & $\mathrm{n}-1$ & $\mathrm{n}$ \\
\hline
\end{tabular}

Figura 8.19: Posição do atributo no vetor de matrizes (mapping). 
A Figura 8.20 ilustra o output gerado após a manipulação dos dados de entrada, por meio do sistema de gerenciamento e das funções de cálculos aplicadas.

\begin{tabular}{|c|c|c|c|c|c|c|}
\hline ID & from & to & $\ldots$ & $\boldsymbol{X}[\mathbf{m}]$ & $\boldsymbol{Y}[\mathrm{m}]$ & $\boldsymbol{Z}$ [m] \\
\hline $\mathbf{1}$ & 1 & 4 & $\ldots$ & $10.551,5368$ & $5.695,1405$ & 297,4962 \\
\hline $\mathbf{2}$ & 4 & 5 & $\ldots$ & $10.551,5386$ & $5.695,1380$ & 297,4899 \\
\hline $\mathbf{3}$ & 2 & 8 & $:$ & $10.551,5352$ & $5.695,1395$ & 297,4956 \\
\hline$:$ & $:$ & $:$ & $:$ & $:$ & $:$ & $:$ \\
\hline $\mathbf{n}-\mathbf{1}$ & $\mathrm{I}_{1,2}$ & 7 & $\ldots$ & $10.551,5298$ & $5.695,1401$ & 297,4902 \\
\hline $\mathbf{n}$ & $:$ & $:$ & $\ldots$ & $:$ & $:$ & $:$ \\
\hline
\end{tabular}

Figura 8.20: Output de dados processados no sistema.

Um sistema de monitoramento geodésico de estruturas produz massivos volumes de dados em diferentes estruturas e modelos. A manipulação desses dados é essencial aos profissionais da área, que acabam esbarrando em aplicações de funcionalidades limitadas, em que não é possível a adaptação e, consequentemente, não atendem por completo as suas necessidades. Desse modo, considera-se como necessária a elaboração de uma padronização das estruturas de armazenamento e de disponibilização dos dados produzidos pelos instrumentos de medição.

Ressalta-se que, o subsistema de processamento permite a integração e a programação de sistemas de alarme em tempo real, uma vez que estejam definidos os parâmetros de controle. Porém, os diferentes sistemas de alarme a serem empregados em um monitoramento, não são o foco deste trabalho.

Assim, para sua validação, o subsistema de processamento foi submetido ao banco de dados gerado pelo monitoramento geodésico de estruturas com controle dos deslocamentos, utilizando três estações totais robóticas. 


\section{Capítulo}

\section{RESULTADOS}

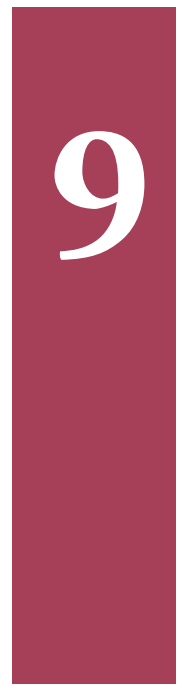

De acordo com os métodos de determinação de coordenadas espaciais distintos, indicados nos capítulos anteriores, foram obtidos os resultados com os testes realizados entre os dias 16/12/2018 e 19/12/2018, o que permitiu fazer análises comparativas entre todos os métodos apresentados nesta tese. Dessa forma, puderam-se obter conclusões e verificar qual desses métodos pode ser utilizado em monitoramento geodésico de estruturas, quando se considera uma área com longas distâncias, em que, por exemplo, os efeitos atmosféricos devem ser considerados. É importante notar que os valores das coordenadas, das precisões e das acurácias estão indicados com excesso de algarismos significativos para realçar a diferença dos valores calculados.

As tabelas, a seguir, apresentam amostras dentro do universo das medições realizadas nesta tese, que permitem a validação do sistema de monitoramento geodésico proposto. Note que a cada instante $t_{0}, t_{1}, t_{2}, \cdots, t_{n}$ das medições em relação ao alvo em deslocamento $\left(X_{P}, Y_{P}, Z_{P}\right)$, as estabilidades de cada base $\left(X_{i}, Y_{i}, Z_{i}\right)$, envolvidas nas observações, foram verificadas.

\subsection{Dados de entrada}

Foram extraídas quatro amostras dentro do universo de medições realizadas pelo sistema de monitoramento geodésico aplicado no Campus 2. E, para melhor organização e representatividade dos dados, levando-se em consideração a magnitude do banco de dados gerado, as amostras foram aleatoriamente escolhidas em períodos diferentes de dias distintos.

A Tabela 9.1 apresenta a amostra 1, com observações realizadas no período da tarde do dia 16/12/2018, considerando-se cinco medições sucessivas, em ciclos de cinco minutos. 
Tabela 9.1: Dados de entrada da amostra 1.

\begin{tabular}{|c|c|c|c|c|c|c|c|c|}
\hline \multirow{2}{*}{$\frac{\text { Tempo }}{[\text { Data/Hora }]}$} & \multirow{2}{*}{$\begin{array}{c}\text { Estaçãoo } \\
{[i]}\end{array}$} & \multirow{2}{*}{$\begin{array}{c}\text { Alvo } \\
{[P]}\end{array}$} & \multirow{2}{*}{$\begin{array}{c}A z_{i P}^{O} \\
{[\text { rad] }}\end{array}$} & \multirow{2}{*}{$\begin{array}{c}\beta_{i P}^{O} \\
{[\mathrm{rad}]}\end{array}$} & \multirow{2}{*}{$\begin{array}{l}d_{i P}^{\prime O} \\
\boldsymbol{m}]\end{array}$} & \multicolumn{3}{|c|}{ Recessão espacial } \\
\hline & & & & & & $X i[m]$ & $Y i[m]$ & $Z i[m]$ \\
\hline 16/12/2018 14:51:09 & TCA & $\mathrm{ROBO}$ & 1,4374637 & $-0,0269145$ & 556,6619 & $9.999,9621$ & $5.621,7174$ & 312,5015 \\
\hline 16/12/2018 14:51:21 & TCRA & $\mathrm{ROBO}$ & 0,6119559 & $-0,0039417$ & 652,8320 & $10.176,5088$ & $5.161,3776$ & 300,0746 \\
\hline 16/12/2018 14:51:22 & TCRP & $\mathrm{ROBO}$ & 0,6700719 & $-0,0028038$ & 887,7790 & $10.000,1673$ & $4.999,9117$ & 300,0088 \\
\hline $16 / 12 / 2018$ 14:56:12 & TCA & $\mathrm{ROBO}$ & 1,4374477 & $-0,0269019$ & 556,6598 & $9.999,9688$ & $5.621,7163$ & 312,5012 \\
\hline 16/12/2018 14:56:14 & TCRA & $\mathrm{ROBO}$ & 0,6119730 & $-0,0039289$ & 652,8340 & $10.176,5084$ & $5.161,3844$ & 300,0796 \\
\hline $16 / 12 / 2018$ 14:56:25 & TCRP & $\mathrm{ROBO}$ & 0,6700480 & $-0,0027859$ & 887,7812 & $10.000,1734$ & $4.999,8871$ & 300,0108 \\
\hline 16/12/2018 15:01:11 & TCA & $\mathrm{ROBO}$ & 1,4374586 & $-0,0269027$ & 556,6611 & $9.999,9935$ & $5.621,7227$ & 312,4958 \\
\hline 16/12/2018 15:01:10 & TCRA & & 0,6119157 & $-0,0039034$ & 652,8348 & $10.176,5166$ & $5.161,3486$ & 300,0734 \\
\hline 16/12/2018 15:01:18 & TCRP & $\mathrm{ROBO}$ & 0,6700771 & $-0,0027950$ & 887,7812 & $10.000,1638$ & $4.999,9134$ & 300,0078 \\
\hline 16/12/2018 15:06:12 & TCA & $\mathrm{ROBO}$ & 1,4374573 & $-0,0269282$ & 556,6590 & $9.999,9846$ & $5.621,7199$ & 312,4980 \\
\hline 16/12/2018 15:06:12 & TCRA & $\mathrm{ROBO}$ & 0,6119331 & $-0,0039239$ & 652,8363 & $10.176,5154$ & $5.161,3587$ & 300,0817 \\
\hline 16/12/2018 15:06:19 & TCRP & $\mathrm{ROBO}$ & 0,6700619 & $-0,0028165$ & 887,7829 & $10.000,1714$ & $4.999,9030$ & 300,0285 \\
\hline 16/12/2018 15:11:11 & TCA & $\mathrm{ROBO}$ & 1,4374544 & $-0,0269219$ & 556,6607 & $9.999,9682$ & $5.621,7168$ & 312,4982 \\
\hline 16/12/2018 15:11:12 & TCRA & $\mathrm{ROBO}$ & 0,6119322 & $-0,0039178$ & 652,8342 & $10.176,5125$ & $5.161,3635$ & 300,0776 \\
\hline 16/12/2018 15:11:27 & TCRP & $\mathrm{ROBO}$ & 0,6700375 & $-0,0028107$ & 887,7807 & $10.000,1705$ & $4.999,8857$ & 300,0263 \\
\hline
\end{tabular}

A Tabela 9.2 apresenta a amostra 2, com observações realizadas no período da madrugada do dia 17/12/2018, considerando-se cinco medições sucessivas, em ciclos de cinco minutos.

Tabela 9.2: Dados de entrada da amostra 2.

\begin{tabular}{|c|c|c|c|c|c|c|c|c|}
\hline Teтpo & Estação & Alvo & $A z_{i P}^{O}$ & $\beta_{i P}^{O}$ & $d_{i P}^{\prime O}$ & \multicolumn{3}{|c|}{ Recessão espacial } \\
\hline [Data/Hora] & {$[i]$} & {$[P]$} & [rad] & [rad] & {$[m]$} & $X i[m]$ & $Y i[m]$ & $Z i[m]$ \\
\hline 17/12/2018 01:01:07 & TCA & $\mathrm{ROBO}$ & 1,4374461 & $-0,0268896$ & 556,6603 & $9.999,9733$ & $5.621,7234$ & 312,4891 \\
\hline 17/12/2018 01:01:13 & TCRA & $\mathrm{ROBO}$ & 0,6119290 & $-0,0038816$ & 652,8397 & $10.176,5121$ & $5.161,3510$ & 300,0562 \\
\hline 17/12/2018 01:01:07 & TCRP & $\mathrm{ROBO}$ & 0,6702186 & $-0,0027773$ & 887,7863 & $10.000,1468$ & $4.999,9850$ & 299,9745 \\
\hline 17/12/2018 01:06:06 & TCA & $\mathrm{ROBO}$ & 1,4374537 & $-0,0268849$ & 556,6609 & $9.999,9780$ & $5.621,7231$ & 312,4892 \\
\hline 17/12/2018 01:06:18 & TCRA & ROBO & 0,6119134 & $-0,0038871$ & 652,8388 & $10.176,5160$ & $5.161,3435$ & 300,0610 \\
\hline 17/12/2018 01:06:12 & TCRP & ROBO & 0,6702069 & $-0,0027733$ & 887,7854 & $10.000,1473$ & $4.999,9876$ & 299,9756 \\
\hline 17/12/2018 01:11:07 & TCA & ROBO & 1,4374785 & $-0,0268981$ & 556,6657 & $9.999,9813$ & $5.621,7239$ & 312,4883 \\
\hline 17/12/2018 01:12:02 & TCRA & $\mathrm{ROBO}$ & 0,6119391 & $-0,0038927$ & 652,8327 & $10.176,5105$ & $5.161,3505$ & 300,0598 \\
\hline 17/12/2018 01:11:06 & TCRP & ROBO & 0,6702008 & $-0,0027639$ & 887,7801 & $10.000,1485$ & $4.999,9460$ & 299,9746 \\
\hline 17/12/2018 01:16:08 & TCA & $\mathrm{ROBO}$ & 1,4374603 & $-0,0268769$ & 556,6617 & $9.999,9803$ & $5.621,7253$ & 312,4894 \\
\hline 17/12/2018 01:16:08 & TCRA & $\mathrm{ROBO}$ & 0,6119152 & $-0,0038710$ & 652,8397 & $10.176,5147$ & $5.161,3414$ & 300,0604 \\
\hline 17/12/2018 01:16:22 & TCRP & ROBO & 0,6701860 & $-0,0027553$ & 887,7863 & $10.000,1474$ & $4.999,9490$ & 299,9708 \\
\hline 17/12/2018 01:21:07 & TCA & ROBO & 1,4374828 & $-0,0268764$ & 556,6673 & $9.999,9766$ & $5.621,7240$ & 312,4859 \\
\hline 17/12/2018 01:21:12 & TCRA & $\mathrm{ROBO}$ & 0,6119392 & $-0,0038704$ & 652,8330 & $10.176,5136$ & $5.161,3460$ & 300,0527 \\
\hline 17/12/2018 01:21:12 & TCRP & ROBO & 0,6702553 & $-0,0027653$ & 887,7803 & $10.000,1449$ & $5.000,0217$ & 299,9649 \\
\hline
\end{tabular}

A Tabela 9.3 apresenta a amostra 3, com observações realizadas no período da manhã do dia 17/12/2018, considerando-se cinco medições sucessivas, em ciclos de cinco minutos. 
Tabela 9.3: Dados de entrada da amostra 3.

\begin{tabular}{|c|c|c|c|c|c|c|c|c|}
\hline Тетро & Estação & Alvo & $A z_{i P}^{O}$ & $\beta_{i P}^{O}$ & $d_{i P}^{\prime O}$ & \multicolumn{3}{|c|}{ Recessão espacial } \\
\hline [Data/Hora] & {$[i]$} & {$[P]$} & [rad] & [rad] & {$[m]$} & $X i[m]$ & $Y i[m]$ & $Z i[m]$ \\
\hline 17/12/2018 06:46:10 & TCA & ROBO & 1,4374913 & $-0,0269031$ & 556,6684 & $9.999,9697$ & $5.621,7236$ & 312,4913 \\
\hline 17/12/2018 06:46:17 & TCRA & $\mathrm{ROBO}$ & 0,6119554 & $-0,0038957$ & 652,8315 & $10.176,5086$ & $5.161,3581$ & 300,0593 \\
\hline 17/12/2018 06:46:19 & TCRP & $\mathrm{ROBO}$ & 0,6701946 & $-0,0027842$ & 887,7791 & $10.000,1497$ & $4.999,9324$ & 299,9855 \\
\hline 17/12/2018 06:51:08 & TCA & $\mathrm{ROBO}$ & 1,4374608 & $-0,0268990$ & 556,6551 & $9.999,9886$ & $5.621,7251$ & 312,4898 \\
\hline 17/12/2018 06:51:08 & TCRA & $\mathrm{ROBO}$ & 0,6119344 & $-0,0038949$ & 652,8378 & $10.176,5111$ & $5.161,3591$ & 300,0603 \\
\hline 17/12/2018 06:51:13 & TCRP & ROBO & 0,6701767 & $-0,0027860$ & 887,7847 & $10.000,1500$ & $4.999,9472$ & 299,9840 \\
\hline 17/12/2018 06:56:10 & TCA & ROBO & 1,4374696 & $-0,0268946$ & 556,6500 & $9.999,9720$ & $5.621,7251$ & 312,4916 \\
\hline 17/12/2018 06:56:09 & TCRA & ROBO & 0,6119140 & $-0,0038900$ & 652,8348 & $10.176,5154$ & $5.161,3387$ & 300,0624 \\
\hline 17/12/2018 06:56:27 & TCRP & $\mathrm{ROBO}$ & 0,6701692 & $-0,0027776$ & 887,7820 & $10.000,1527$ & $4.999,9208$ & 299,9869 \\
\hline 17/12/2018 07:01:14 & TCA & $\mathrm{ROBO}$ & 1,4374538 & $-0,0269090$ & 556,6606 & $9.999,9838$ & $5.621,7250$ & 312,4908 \\
\hline 17/12/2018 07:01:07 & TCRA & $\mathrm{ROBO}$ & 0,6119057 & $-0,0039020$ & 652,8390 & $10.176,5161$ & $5.161,3444$ & 300,0637 \\
\hline 17/12/2018 07:01:14 & TCRP & $\mathrm{ROBO}$ & 0,6701613 & $-0,0027734$ & 887,7854 & $10.000,1532$ & $4.999,9118$ & 299,9877 \\
\hline 17/12/2018 07:06:10 & TCA & $\mathrm{ROBO}$ & 1,4374647 & $-0,0269152$ & 556,6653 & $9.999,9798$ & $5.621,7223$ & 312,4904 \\
\hline 17/12/2018 07:06:19 & TCRA & $\mathrm{ROBO}$ & 0,6119330 & $-0,0039025$ & 652,8341 & $10.176,5155$ & $5.161,3458$ & 300,0623 \\
\hline 17/12/2018 07:06:08 & TCRP & ROBO & 0,6701928 & $-0,0027758$ & 887,7815 & $10.000,1510$ & $4.999,9435$ & 299,9897 \\
\hline
\end{tabular}

A Tabela 9.4 apresenta a amostra 4, com observações realizadas no período da noite do dia 18/12/2018, considerando-se cinco medições sucessivas, em ciclos de cinco minutos.

Tabela 9.4: Dados de entrada da amostra 4.

\begin{tabular}{|c|c|c|c|c|c|c|c|c|}
\hline Tempo & Estação & Alvo & $A z_{i P}^{O}$ & $\beta_{i P}^{O}$ & $d_{i P}^{\prime O}$ & \multicolumn{3}{|c|}{ Recessão espacial } \\
\hline [Data/Hora] & {$[i]$} & {$[P]$} & [rad] & [rad] & {$[m]$} & $X i[m]$ & $Y i[m]$ & $Z i[m]$ \\
\hline 18/12/2018 21:01:06 & TCA & $\mathrm{ROBO}$ & 1,4374856 & $-0,0268871$ & 556,6695 & $9.999,9799$ & $5.621,7231$ & 312,4904 \\
\hline 18/12/2018 21:01:08 & TCRA & ROBO & 0,6119364 & $-0,0038865$ & 652,8302 & $10.176,5193$ & $5.161,3406$ & 300,0652 \\
\hline 18/12/2018 21:01:33 & TCRP & ROBO & 0,6701435 & $-0,0027935$ & 887,7851 & $10.000,1516$ & $4.999,8940$ & 299,9885 \\
\hline 18/12/2018 21:06:08 & TCA & ROBO & 1,4374861 & $-0,0268854$ & 556,6682 & $9.999,9741$ & $5.621,7217$ & 312,4907 \\
\hline 18/12/2018 21:06:14 & TCRA & $\mathrm{ROBO}$ & 0,6119528 & $-0,0038915$ & 652,8302 & $10.176,5145$ & $5.161,3515$ & 300,0645 \\
\hline 18/12/2018 21:06:42 & TCRP & $\mathrm{ROBO}$ & 0,6701353 & $-0,0027809$ & 887,7784 & $10.000,1550$ & $4.999,8441$ & 299,9903 \\
\hline 18/12/2018 21:11:06 & TCA & $\mathrm{ROBO}$ & 1,4374444 & $-0,0269075$ & 556,6591 & $9.999,9800$ & $5.621,7229$ & 312,4911 \\
\hline 18/12/2018 21:11:11 & TCRA & ROBO & 0,6119145 & $-0,0039022$ & 652,8384 & $10.176,5126$ & $5.161,3510$ & 300,0648 \\
\hline 18/12/2018 21:11:18 & TCRP & $\mathrm{ROBO}$ & 0,6701155 & $-0,0027843$ & 887,7784 & $10.000,1570$ & $4.999,8133$ & 299,9927 \\
\hline 18/12/2018 21:16:07 & TCA & ROBO & 1,4374689 & $-0,0269031$ & 556,6653 & $9.999,9858$ & $5.621,7243$ & 312,4908 \\
\hline 18/12/2018 21:16:17 & TCRA & ROBO & 0,6119323 & $-0,0039034$ & 652,8322 & $10.176,5164$ & $5.161,3463$ & 300,0634 \\
\hline 18/12/2018 21:16:20 & TCRP & $\mathrm{ROBO}$ & 0,6700752 & $-0,0028027$ & 887,7853 & $10.000,1602$ & $4.999,9104$ & 299,9931 \\
\hline 18/12/2018 21:21:09 & TCA & $\mathrm{ROBO}$ & 1,4374935 & $-0,0268920$ & 556,6688 & $9.999,9833$ & $5.621,7246$ & 312,4901 \\
\hline 18/12/2018 21:21:13 & TCRA & $\mathrm{ROBO}$ & 0,6119480 & $-0,0038971$ & 652,8297 & $10.176,5134$ & $5.161,3494$ & 300,0625 \\
\hline 18/12/2018 21:21:30 & TCRP & ROBO & 0,6700675 & $-0,0027917$ & 887,7796 & $10.000,1650$ & $4.999,8915$ & 299,9913 \\
\hline
\end{tabular}

Para a correção dos efeitos atmosféricos, foram coletadas na mesma periodicidade das medições, a temperatura e pressão, para que, assim, as devidas correções fossem aplicadas.

A variação da temperatura $\mathrm{em}^{\circ} \mathrm{C}$, ocorrida no período de monitoramento, pode ser vista na Figura 9.1. 


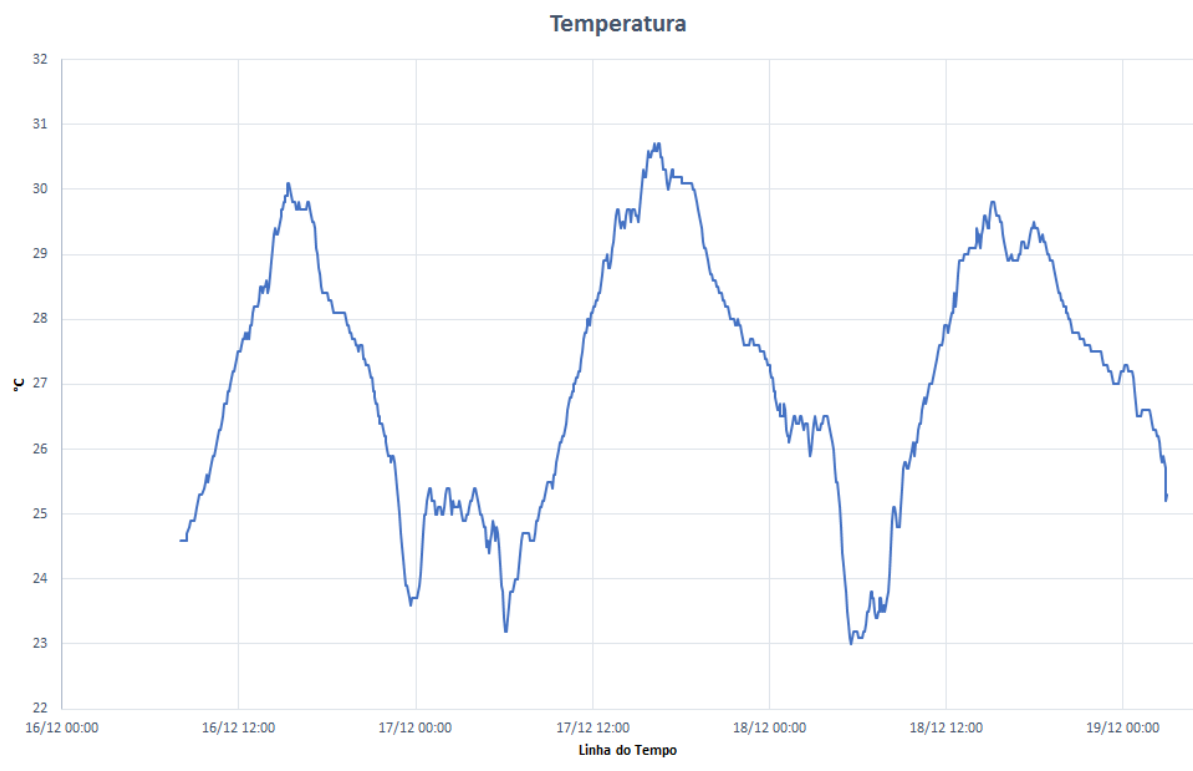

Figura 9.1: Variação da temperatura.

E, a variação da pressão em mbar, ocorrida no período de monitoramento, pode ser vista na Figura 9.2.

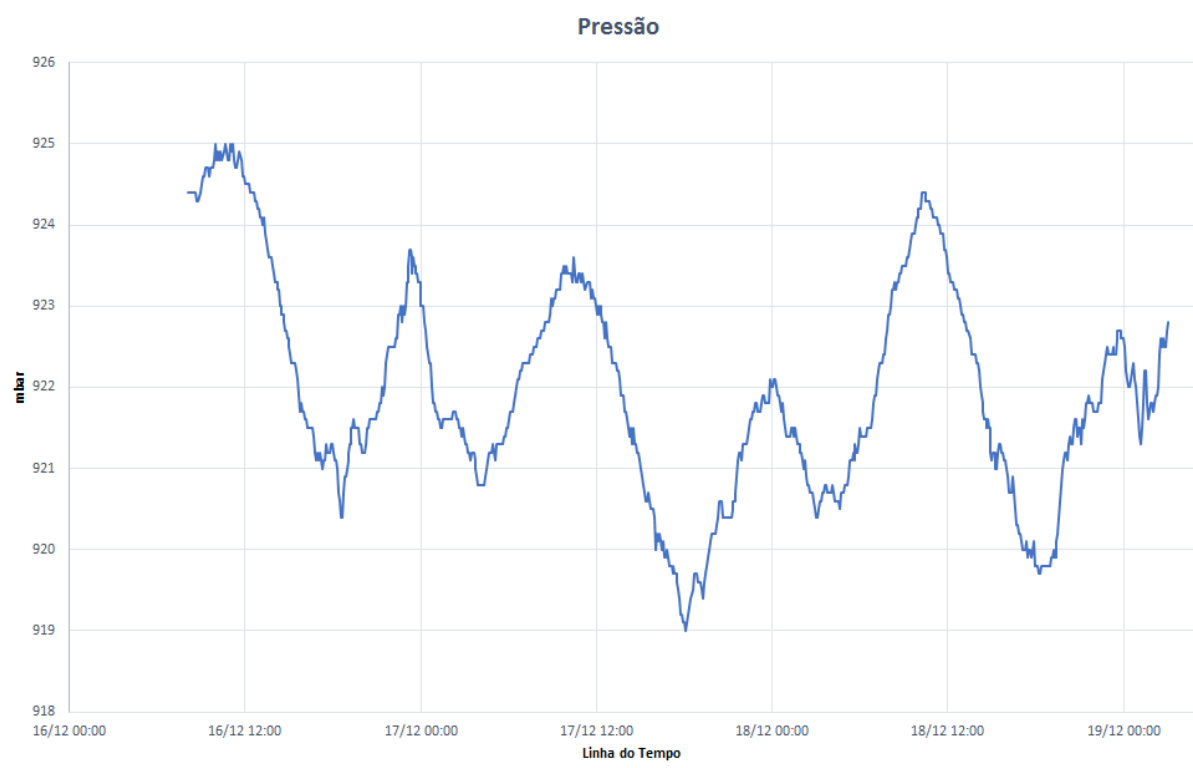

Figura 9.2: Variação da pressão atmosférica.

Na sequência, são apresentados os dados coletados das coordenadas espaciais do alvo controlado, pelo braço robótico, nos mesmos períodos dos dados de entrada coletados pelas estações totais robóticas, tudo para efeito de análises comparativas.

A Tabela 9.5 apresenta as coordenadas espaciais posicionadas pelo robô na amostra 1, no período da tarde do dia 16/12/2018. 
Tabela 9.5: Coordenadas espaciais posicionadas pelo robô na amostra 1.

\begin{tabular}{|c|c|c|c|}
\hline Tempo & \multicolumn{3}{|c|}{ Coordenadas do alvo } \\
\hline [Data/Hora] & $X[m]$ & $Y P[m]$ & $Z P[m]$ \\
\hline 16/12/2018 14:50:00 & $10.551,5144$ & $5.695,7206$ & 297,5167 \\
\hline 16/12/2018 14:55:00 & $10.551,5152$ & $5.695,7207$ & 297,5307 \\
\hline 16/12/2018 15:00:00 & $10.551,5211$ & $5.695,7192$ & 297,5349 \\
\hline 16/12/2018 15:05:00 & $10.551,5190$ & $5.695,7238$ & 297,5262 \\
\hline 16/12/2018 15:10:00 & $10.551,5092$ & $5.695,7202$ & 297,5279 \\
\hline
\end{tabular}

A Tabela 9.6 apresenta as coordenadas espaciais posicionadas pelo robô na amostra 2, no período da madrugada do dia 17/12/2018.

Tabela 9.6: Coordenadas espaciais posicionadas pelo robô na amostra 2.

\begin{tabular}{cccc}
\hline Tempo & \multicolumn{3}{c}{ Coordenadas do alvo } \\
\hline [Data/Hora] & $X[\boldsymbol{m}]$ & $Y[\boldsymbol{m}]$ & $Z[\boldsymbol{m}]$ \\
\hline 17/12/2018 01:00:00 & $10.551,5421$ & $5.695,7224$ & 297,5248 \\
$17 / 12 / 2018$ 01:05:00 & $10.551,5434$ & $5.695,7240$ & 297,5221 \\
$17 / 12 / 201801: 10: 00$ & $10.551,5425$ & $5.695,7026$ & 297,5283 \\
$17 / 12 / 2018$ 01:15:00 & $10.551,5408$ & $5.695,7133$ & 297,5287 \\
$17 / 12 / 2018$ 01:20:00 & $10.551,5547$ & $5.695,7146$ & 297,5251 \\
\hline
\end{tabular}

A Tabela 9.7 apresenta as coordenadas espaciais posicionadas pelo robô na amostra 3, no período da manhã do dia 17/12/2018.

Tabela 9.7: Coordenadas espaciais posicionadas pelo robô na amostra 3.

\begin{tabular}{cccc}
\hline Tempo & \multicolumn{3}{c}{ Coordenadas do alvo } \\
\hline [Data/Hora $]$ & $X[\boldsymbol{m}]$ & $Y[\boldsymbol{m}]$ & $Z[\boldsymbol{m}]$ \\
\hline $17 / 12 / 2018$ 06:45:00 & $10.551,5431$ & $5.695,6967$ & 297,5200 \\
$17 / 12 / 2018$ 06:50:00 & $10.551,5401$ & $5.695,7150$ & 297,5206 \\
$17 / 12 / 201806: 55: 00$ & $10.551,5271$ & $5.695,7012$ & 297,5258 \\
$17 / 12 / 201807: 00: 00$ & $10.551,5342$ & $5.695,7079$ & 297,5188 \\
$17 / 12 / 201807: 05: 00$ & $10.551,5445$ & $5.695,7039$ & 297,5252 \\
\hline
\end{tabular}

A Tabela 9.8 apresenta as coordenadas espaciais posicionadas pelo robô na amostra 4, no período da noite do dia 18/12/2018. 
Tabela 9.8: Coordenadas espaciais posicionadas pelo robô na amostra 4.

\begin{tabular}{cccc}
\hline Tempo & \multicolumn{3}{c}{ Coordenadas do alvo } \\
\hline [Data/Hora $]$ & $X[\boldsymbol{m}]$ & $Y[\boldsymbol{m}]$ & $Z[\boldsymbol{m}]$ \\
\hline $18 / 12 / 2018$ 21:00:00 & $10.551,5365$ & $5.695,6933$ & 297,5274 \\
$18 / 12 / 2018$ 21:05:00 & $10.551,5314$ & $5.695,6746$ & 297,5298 \\
$18 / 12 / 2018$ 21:10:00 & $10.551,5218$ & $5.695,6850$ & 297,5195 \\
$18 / 12 / 2018$ 21:15:00 & $10.551,5213$ & $5.695,7143$ & 297,5143 \\
$18 / 12 / 201821: 20: 00$ & $10.551,5221$ & $5.695,7040$ & 297,5220 \\
\hline
\end{tabular}

A Figura 9.3 mostra a visualização gráfica da variação das coordenadas espaciais do alvo controlado, considerando-se o agrupamento de todas as amostras que foram aleatoriamente selecionadas.

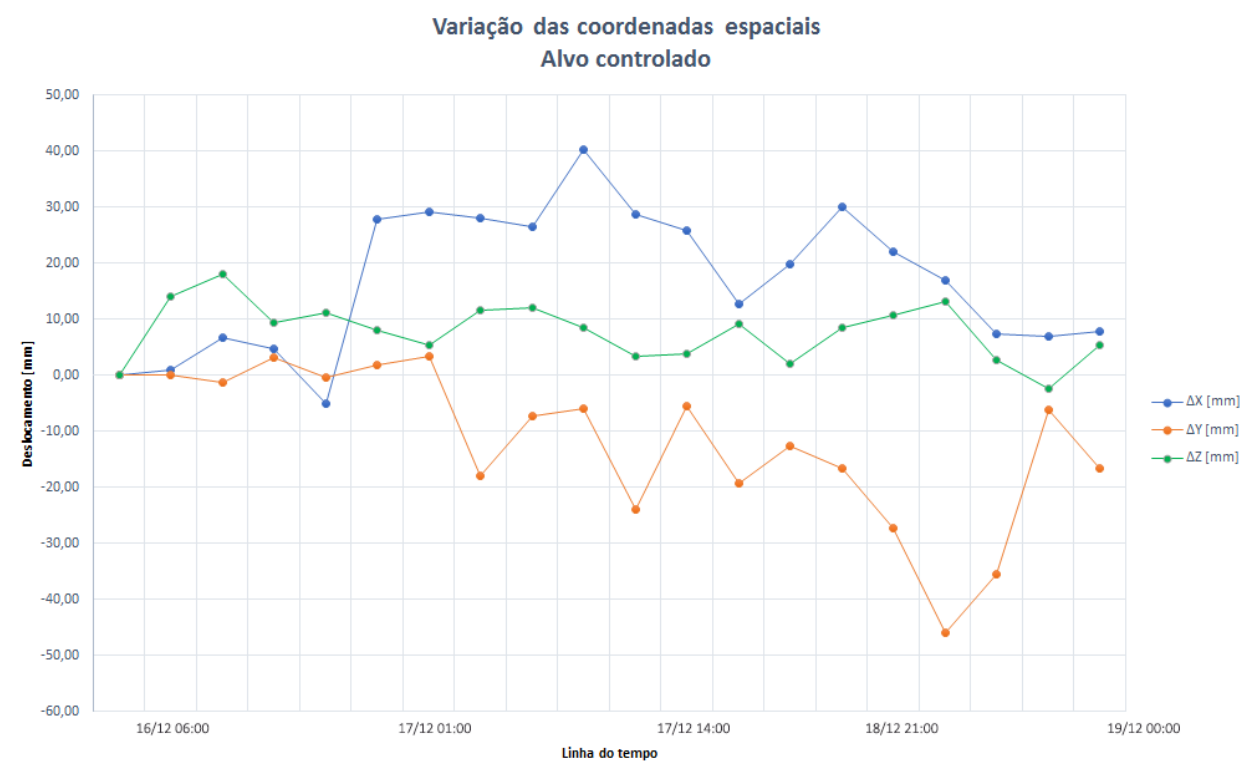

Figura 9.3: Variação das coordenadas espaciais do alvo controlado pelo braço robótico.

A Figura 9.4 apresenta as movimentações realizadas pelo robô em cada eixo. Dessa forma, é possível visualizar, graficamente, as posições separadamente de $X_{P}$ na Figura 9.4a, de $Y_{P}$ na Figura 9.4b e de $Z_{P}$ na Figura 9.4c das amostras consideradas.

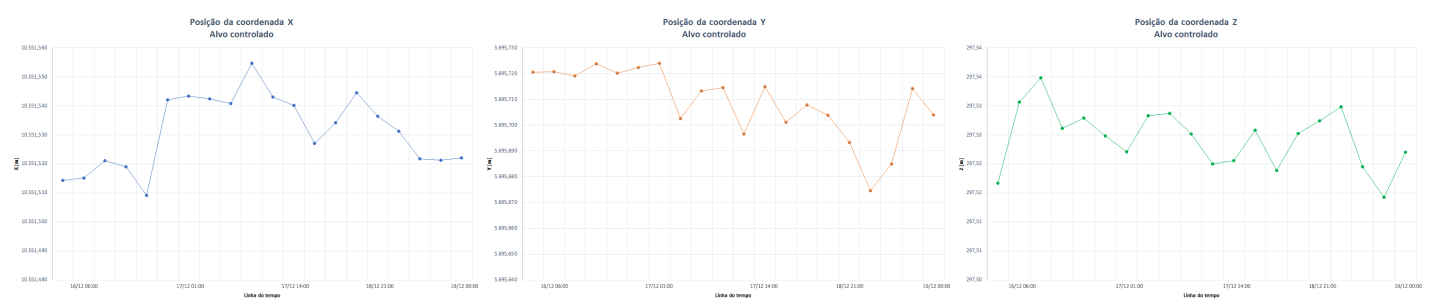
(a) Coordenada $X_{P}$.
(b) Coordenada $Y_{P}$.
(c) Coordenada $Z_{P}$.

Figura 9.4: Posições das coordenadas $X_{P}, Y_{P}$ e $Z_{P}$ (braço robótico). 


\subsection{Resultados dos métodos}

Os resultados obtidos pela média simples entre as coordenadas obtidas com as medições das três estações totais robóticas e determinadas pelo método polar, aplicado às amostras dos dados de entrada, podem ser vistos na Tabela 9.9.

Tabela 9.9: Coordenadas espaciais determinadas pela média do método polar.

\begin{tabular}{|c|c|c|c|}
\hline \multirow{2}{*}{$\frac{\text { Tempo }}{[\text { Data/Hora] }}$} & \multicolumn{3}{|c|}{ Coordenadas do alvo } \\
\hline & $X[m]$ & $Y[m]$ & $Z[\mathrm{~m}]$ \\
\hline 16/12/2018 14:51:09 & $10.551,5117$ & $5.695,7184$ & 297,5140 \\
\hline 16/12/2018 14:56:12 & $10.551,5131$ & $5.695,7184$ & 297,5267 \\
\hline 16/12/2018 15:01:11 & $10.551,5183$ & $5.695,7174$ & 297,5245 \\
\hline 16/12/2018 15:06:12 & $10.551,5167$ & $5.695,7179$ & 297,5194 \\
\hline 16/12/2018 15:11:11 & $10.551,5039$ & $5.695,7168$ & 297,5215 \\
\hline 17/12/2018 01:01:07 & $10.551,5411$ & $5.695,7196$ & 297,5179 \\
\hline 17/12/2018 01:06:06 & $10.551,5387$ & $5.695,7202$ & 297,5207 \\
\hline 17/12/2018 01:11:07 & $10.551,5413$ & $5.695,6993$ & 297,5188 \\
\hline 17/12/2018 01:16:08 & $10.551,5353$ & $5.695,7102$ & 297,5293 \\
\hline 17/12/2018 01:21:07 & $10.551,5531$ & $5.695,7125$ & 297,5208 \\
\hline 17/12/2018 06:46:10 & $10.551,5395$ & $5.695,6936$ & 297,5156 \\
\hline 17/12/2018 06:51:08 & $10.551,5360$ & $5.695,7134$ & 297,5155 \\
\hline 17/12/2018 06:56:10 & $10.551,5249$ & $5.695,6984$ & 297,5222 \\
\hline 17/12/2018 07:01:14 & $10.551,5304$ & $5.695,7051$ & 297,5184 \\
\hline 17/12/2018 07:06:10 & $10.551,5404$ & $5.695,7019$ & 297,5165 \\
\hline 18/12/2018 21:01:06 & $10.551,5331$ & $5.695,6888$ & 297,5205 \\
\hline 18/12/2018 21:06:08 & $10.551,5299$ & $5.695,6729$ & 297,5239 \\
\hline 18/12/2018 21:11:06 & $10.551,5180$ & $5.695,6808$ & 297,5176 \\
\hline 18/12/2018 21:16:07 & $10.551,5189$ & $5.695,7131$ & 297,5122 \\
\hline 18/12/2018 21:21:09 & $10.551,5199$ & $5.695,7009$ & 297,5177 \\
\hline
\end{tabular}


A Figura 9.5 mostra a visualização gráfica da variação das coordenadas espaciais detectadas pelo método polar com média.

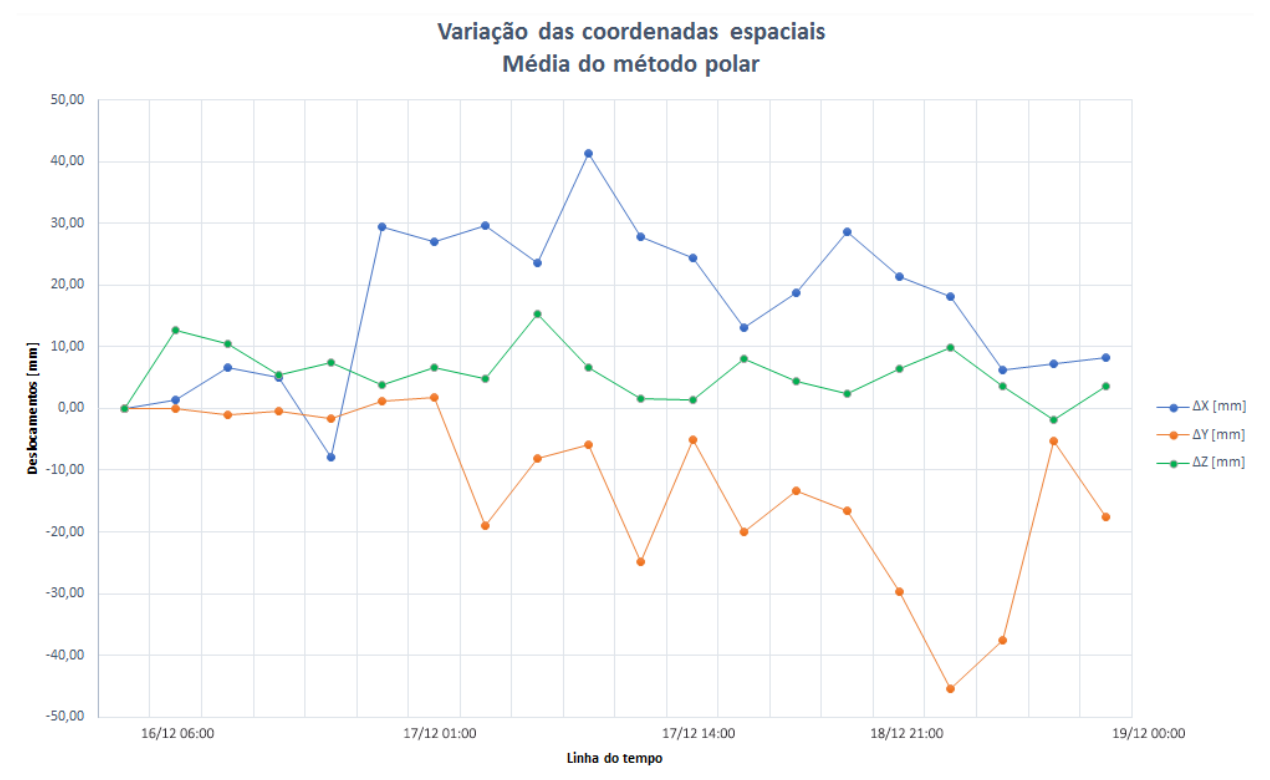

Figura 9.5: Variação das coordenadas espaciais do método polar com média.

A Figura 9.6 apresenta a determinação das coordenadas pelo método polar com média. Dessa forma, é possível visualizar, graficamente, as posições separadamente de $X_{P}$ na Figura 9.6a, de $Y_{P}$ na Figura 9.6b e de $Z_{P}$ na Figura 9.6c.
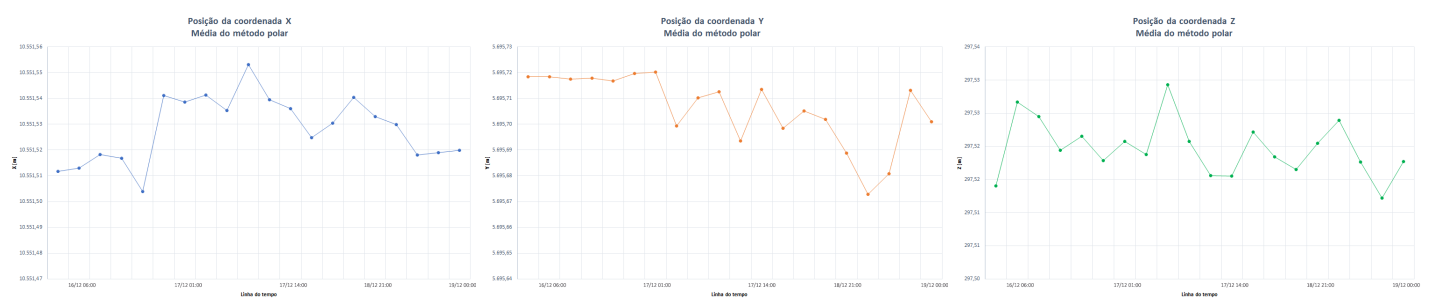
(a) Coordenada $X_{P}$.
(b) Coordenada $Y_{P}$.
(c) Coordenada $Z_{P}$.

Figura 9.6: Posições das coordenadas $X_{P}, Y_{P}$ e $Z_{P}$ (polar com média). 
Os resultados obtidos pelo método polar com ajustamento das observações dos dados medidos das três estações totais robóticas podem ser vistos na Tabela 9.10.

Tabela 9.10: Coordenadas espaciais determinadas pelo método polar com ajustamento.

\begin{tabular}{|c|c|c|c|c|c|c|c|}
\hline \multirow{2}{*}{$\begin{array}{c}\text { Tempo } \\
\text { Data/Hora] }\end{array}$} & \multicolumn{3}{|c|}{ Coordenadas do alvo } & \multicolumn{4}{|c|}{ Precisões } \\
\hline & $X[m]$ & $Y[m]$ & $Z[m]$ & $\sigma_{X}[\mathbf{m m}]$ & $\sigma_{Y}[\mathbf{m m}]$ & $\sigma_{Z}[\mathbf{m m}]$ & $\sigma_{X Y Z}[\mathbf{m m}]$ \\
\hline 16/12/2018 14:51:09 & $10.551,5117$ & $5.695,7184$ & 297,5140 & 1,98004 & 1,60867 & 2,48496 & 3,56137 \\
\hline 16/12/2018 14:56:12 & $10.551,5131$ & $5.695,7184$ & 297,5267 & 1,98008 & 1,60863 & 2,48496 & 3,56138 \\
\hline 16/12/2018 15:01:11 & $10.551,5183$ & $5.695,7174$ & 297,5245 & 1,98004 & 1,60868 & 2,48496 & 3,56138 \\
\hline 16/12/2018 15:06:12 & $10.551,5167$ & $5.695,7179$ & 297,5194 & 1,98006 & 1,60866 & 2,48497 & 3,56139 \\
\hline 16/12/2018 15:11:11 & $10.551,5039$ & $5.695,7168$ & 297,5215 & 1,98009 & 1,60861 & 2,48496 & 3,56138 \\
\hline 17/12/2018 01:01:07 & $10.551,5411$ & $5.695,7196$ & 297,5179 & 1,97985 & 1,60894 & 2,48498 & 3,56140 \\
\hline 17/12/2018 01:06:06 & $10.551,5387$ & $5.695,7202$ & 297,5207 & 1,97986 & 1,60892 & 2,48497 & 3,56140 \\
\hline 17/12/2018 01:11:07 & $10.551,5413$ & $5.695,6993$ & 297,5188 & 1,97986 & 1,60890 & 2,48496 & 3,56138 \\
\hline 17/12/2018 01:16:08 & $10.551,5353$ & $5.695,7102$ & 297,5293 & 1,97989 & 1,60888 & 2,48498 & 3,56140 \\
\hline 17/12/2018 01:21:07 & $10.551,5531$ & $5.695,7125$ & 297,5208 & 1,97978 & 1,60900 & 2,48496 & 3,56138 \\
\hline 17/12/2018 06:46:10 & $10.551,5395$ & $5.695,6936$ & 297,5156 & 1,97987 & 1,60889 & 2,48496 & 3,56137 \\
\hline 17/12/2018 06:51:08 & $10.551,5360$ & $5.695,7135$ & 297,5155 & 1,97990 & 1,60886 & 2,48497 & 3,56140 \\
\hline 17/12/2018 06:56:10 & $10.551,5249$ & $5.695,6984$ & 297,5222 & 1,97991 & 1,60885 & 2,48496 & 3,56139 \\
\hline 17/12/2018 07:01:14 & $10.551,5304$ & $5.695,7052$ & 297,5184 & 1,97993 & 1,60884 & 2,48497 & 3,56140 \\
\hline 17/12/2018 07:06:10 & $10.551,5404$ & $5.695,7019$ & 297,5165 & 1,97987 & 1,60889 & 2,48496 & 3,56138 \\
\hline 18/12/2018 21:01:06 & $10.551,5331$ & $5.695,6888$ & 297,5205 & 1,97995 & 1,60881 & 2,48497 & 3,56140 \\
\hline 18/12/2018 21:06:08 & $10.551,5299$ & $5.695,6729$ & 297,5239 & 1,97995 & 1,60878 & 2,48495 & 3,56137 \\
\hline 18/12/2018 21:11:06 & $10.551,5180$ & $5.695,6808$ & 297,5176 & 1,97998 & 1,60875 & 2,48495 & 3,56137 \\
\hline 18/12/2018 21:16:07 & $10.551,5189$ & $5.695,7131$ & 297,5122 & 1,98005 & 1,60869 & 2,48497 & 3,56140 \\
\hline 18/12/2018 21:21:09 & $10.551,5199$ & $5.695,7009$ & 297,5177 & 1,98005 & 1,60866 & 2,48496 & 3,56138 \\
\hline
\end{tabular}

A Figura 9.7 mostra a visualização gráfica da variação das coordenadas espaciais detectadas pelo método polar com ajustamento das observações.

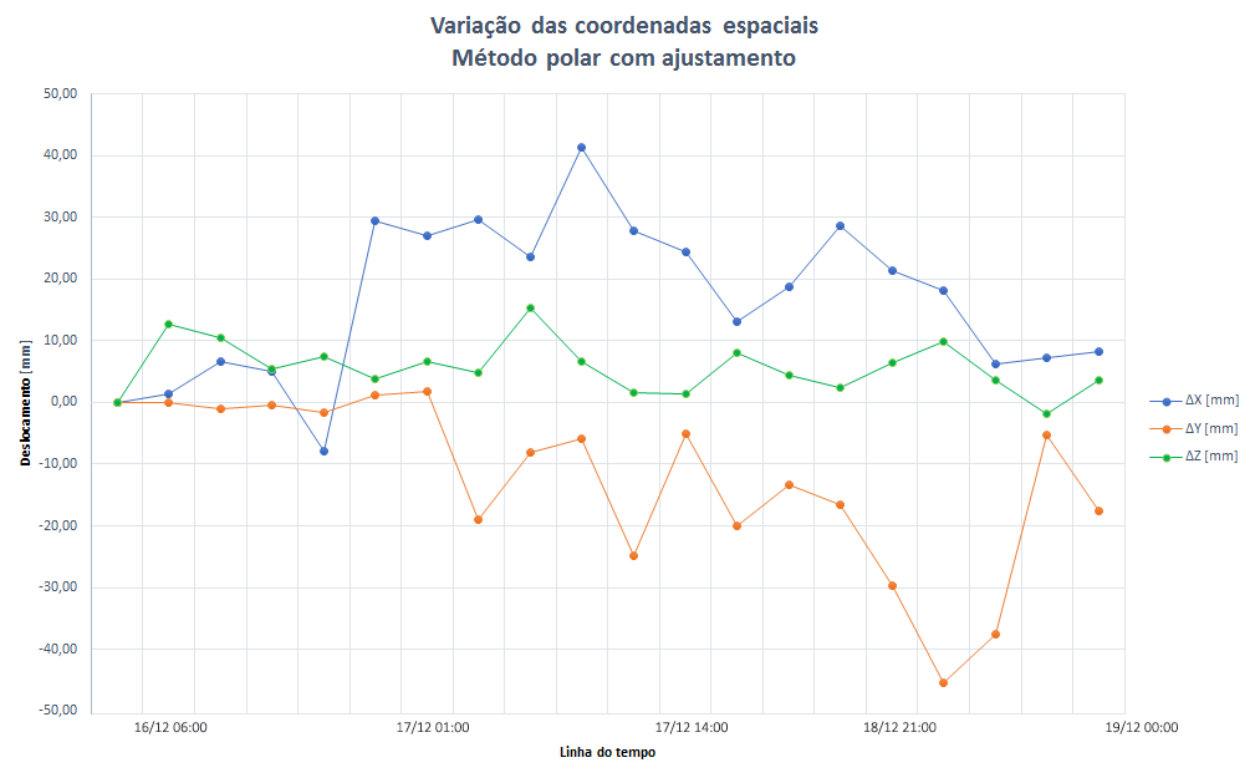

Figura 9.7: Variação das coordenadas espaciais do método polar com ajustamento. 
A Figura 9.8 apresenta a determinação das coordenadas pelo método polar com ajustamento das observações. Dessa forma, é possível visualizar, graficamente, as posições separadamente de $X_{P}$ na Figura 9.8a, de $Y_{P}$ na Figura 9.8b e de $Z_{P}$ na Figura 9.8c.

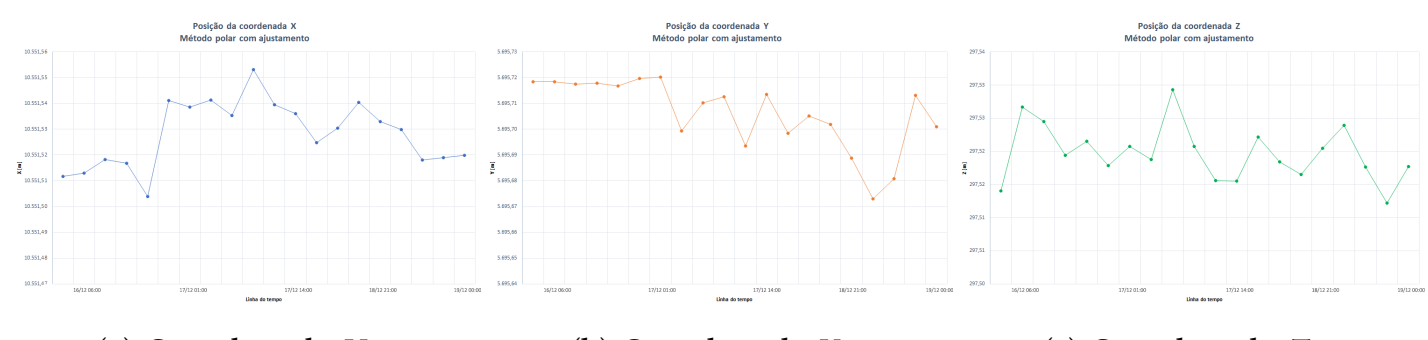
(a) Coordenada $X_{P}$.
(b) Coordenada $Y_{P}$.
(c) Coordenada $Z_{P}$.

Figura 9.8: Posições das coordenadas $X_{P}, Y_{P}$ e $Z_{P}$ (polar com ajustamento).

Os resultados obtidos pela média simples entre as coordenadas obtidas com as medições das três estações totais robóticas, tomadas duas a duas, e determinadas pelo método de interseção espacial trigonométrico, podem ser vistos na Tabela 9.11.

Tabela 9.11: Coordenadas espaciais determinadas pelo método de interseção espacial trigonométrico.

\begin{tabular}{|c|c|c|c|}
\hline \multirow{2}{*}{$\frac{\text { Tempo }}{[\text { Data/Hora] }}$} & \multicolumn{3}{|c|}{ Coordenadas do alvo } \\
\hline & $X[m]$ & $Y[m]$ & $Z[m]$ \\
\hline 16/12/2018 14:51:09 & $10.551,5669$ & $5.695,7866$ & 297,5136 \\
\hline 16/12/2018 14:56:12 & $10.551,6021$ & $5.695,8292$ & 297,5261 \\
\hline 16/12/2018 15:01:11 & $10.551,5540$ & $5.695,7713$ & 297,5244 \\
\hline 16/12/2018 15:06:12 & $10.551,5746$ & $5.695,7961$ & 297,5191 \\
\hline 16/12/2018 15:11:11 & $10.551,5851$ & $5.695,8246$ & 297,5211 \\
\hline 17/12/2018 01:01:07 & $10.551,3750$ & $5.695,4622$ & 297,5186 \\
\hline 17/12/2018 01:06:06 & $10.551,5851$ & $5.695,8220$ & 297,5154 \\
\hline 17/12/2018 01:11:07 & $10.551,5986$ & $5.695,8075$ & 297,5169 \\
\hline 17/12/2018 01:16:08 & $10.551,3794$ & $5.695,4538$ & 297,5264 \\
\hline 17/12/2018 01:21:07 & $10.551,4192$ & $5.695,5412$ & 297,5162 \\
\hline 17/12/2018 06:46:10 & $10.551,3724$ & $5.695,4470$ & 297,5162 \\
\hline 17/12/2018 06:51:08 & $10.551,4024$ & $5.695,5184$ & 297,5160 \\
\hline 17/12/2018 06:56:10 & $10.551,3748$ & $5.695,4698$ & 297,5225 \\
\hline 17/12/2018 07:01:14 & $10.551,3495$ & $5.695,4435$ & 297,5191 \\
\hline 17/12/2018 07:06:10 & $10.551,3926$ & $5.695,4820$ & 297,5169 \\
\hline 18/12/2018 21:01:06 & $10.551,4551$ & $5.695,5698$ & 297,5207 \\
\hline 18/12/2018 21:06:08 & $10.551,3822$ & $5.695,4491$ & 297,5243 \\
\hline 18/12/2018 21:11:06 & $10.551,2815$ & $5.695,3329$ & 297,5185 \\
\hline 18/12/2018 21:16:07 & $10.551,6075$ & $5.695,8354$ & 297,5117 \\
\hline 18/12/2018 21:21:09 & $10.551,6001$ & $5.695,8132$ & 297,5174 \\
\hline
\end{tabular}


A Figura 9.9 mostra a visualização gráfica da variação das coordenadas espaciais detectadas pelo método de interseção espacial trigonométrico.

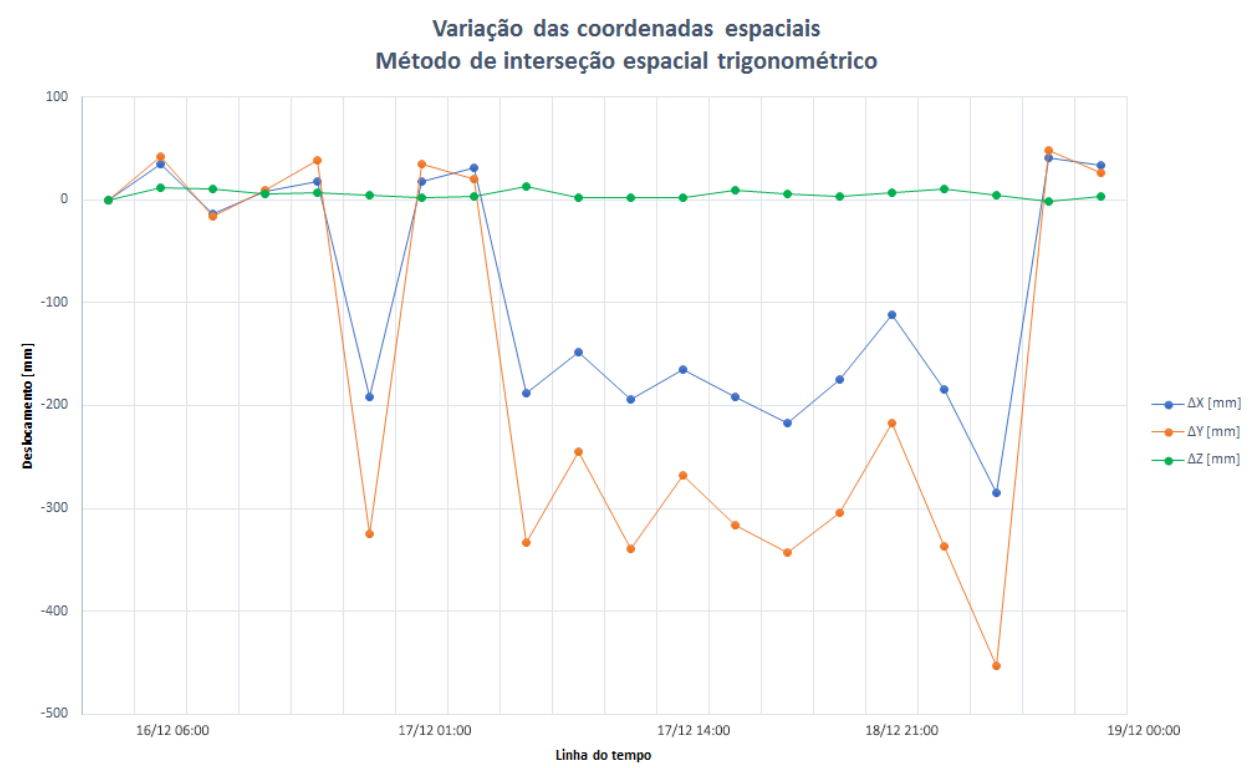

Figura 9.9: Variação das coordenadas espaciais do método de interseção espacial trigonométrico.

A Figura 9.10 apresenta a determinação das coordenadas pelo método de interseção espacial trigonométrico. Dessa forma, é possível visualizar, graficamente, as posições separadamente de $X_{P}$ na Figura 9.10a, de $Y_{P}$ na Figura 9.10b e de $Z_{P}$ na Figura 9.10c.

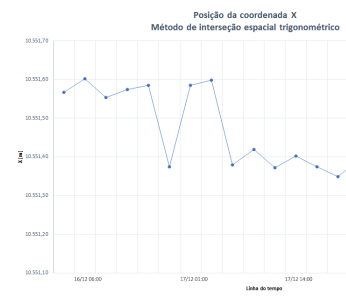

(a) Coordenada $X_{P}$.

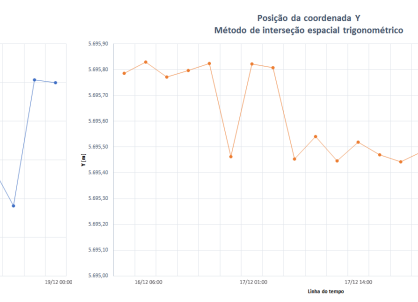

(b) Coordenada $Y_{P}$.

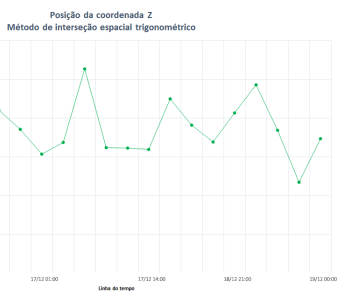

(c) Coordenada $Z_{P}$.

Figura 9.10: Posições das coordenadas $X_{P}, Y_{P}$ e $Z_{P}$ (interseção espacial trigonométrico). 
Os resultados obtidos pela média simples entre as coordenadas obtidas com as medições das três estações totais robóticas, tomadas duas a duas, e determinadas pelo método do ponto médio da interseção espacial, podem ser vistos na Tabela 9.12 .

Tabela 9.12: Coordenadas espaciais determinadas pelo método do ponto médio da interseção espacial.

\begin{tabular}{|c|c|c|c|}
\hline \multirow{2}{*}{$\frac{\text { Tempo }}{[\text { Data/Hora] }}$} & \multicolumn{3}{|c|}{ Coordenadas do alvo } \\
\hline & $X[m]$ & $Y[m]$ & $Z[m]$ \\
\hline 16/12/2018 14:51:09 & $10.551,5658$ & $5.695,7852$ & 297,5138 \\
\hline 16/12/2018 14:56:12 & $10.551,6004$ & $5.695,8272$ & 297,5263 \\
\hline 16/12/2018 15:01:11 & $10.551,5538$ & $5.695,7711$ & 297,5245 \\
\hline 16/12/2018 15:06:12 & $10.551,5737$ & $5.695,7952$ & 297,5193 \\
\hline 16/12/2018 15:11:11 & $10.551,5840$ & $5.695,8236$ & 297,5213 \\
\hline 17/12/2018 01:01:07 & $10.551,3845$ & $5.695,4830$ & 297,5178 \\
\hline 17/12/2018 01:06:06 & $10.551,4034$ & $5.695,5205$ & 297,5208 \\
\hline 17/12/2018 01:11:07 & $10.551,3676$ & $5.695,4479$ & 297,5189 \\
\hline 17/12/2018 01:16:08 & $10.551,3913$ & $5.695,4986$ & 297,5293 \\
\hline 17/12/2018 01:21:07 & $10.551,3935$ & $5.695,4791$ & 297,5209 \\
\hline 17/12/2018 06:46:10 & $10.551,3726$ & $5.695,4472$ & 297,5157 \\
\hline 17/12/2018 06:51:08 & $10.551,4030$ & $5.695,5191$ & 297,5156 \\
\hline 17/12/2018 06:56:10 & $10.551,3749$ & $5.695,4700$ & 297,5221 \\
\hline 17/12/2018 07:01:14 & $10.551,3487$ & $5.695,4427$ & 297,5186 \\
\hline 17/12/2018 07:06:10 & $10.551,3916$ & $5.695,4811$ & 297,5165 \\
\hline 18/12/2018 21:01:06 & $10.551,4567$ & $5.695,5715$ & 297,5204 \\
\hline 18/12/2018 21:06:08 & $10.551,3825$ & $5.695,4494$ & 297,5238 \\
\hline 18/12/2018 21:11:06 & $10.551,2813$ & $5.695,3328$ & 297,5177 \\
\hline 18/12/2018 21:16:07 & $10.551,6084$ & $5.695,8363$ & 297,5120 \\
\hline 18/12/2018 21:21:09 & $10.551,6006$ & $5.695,8136$ & 297,5176 \\
\hline
\end{tabular}


Figura 9.11 mostra a visualização gráfica da variação das coordenadas espaciais detectadas pelo método do ponto médio da interseção espacial.

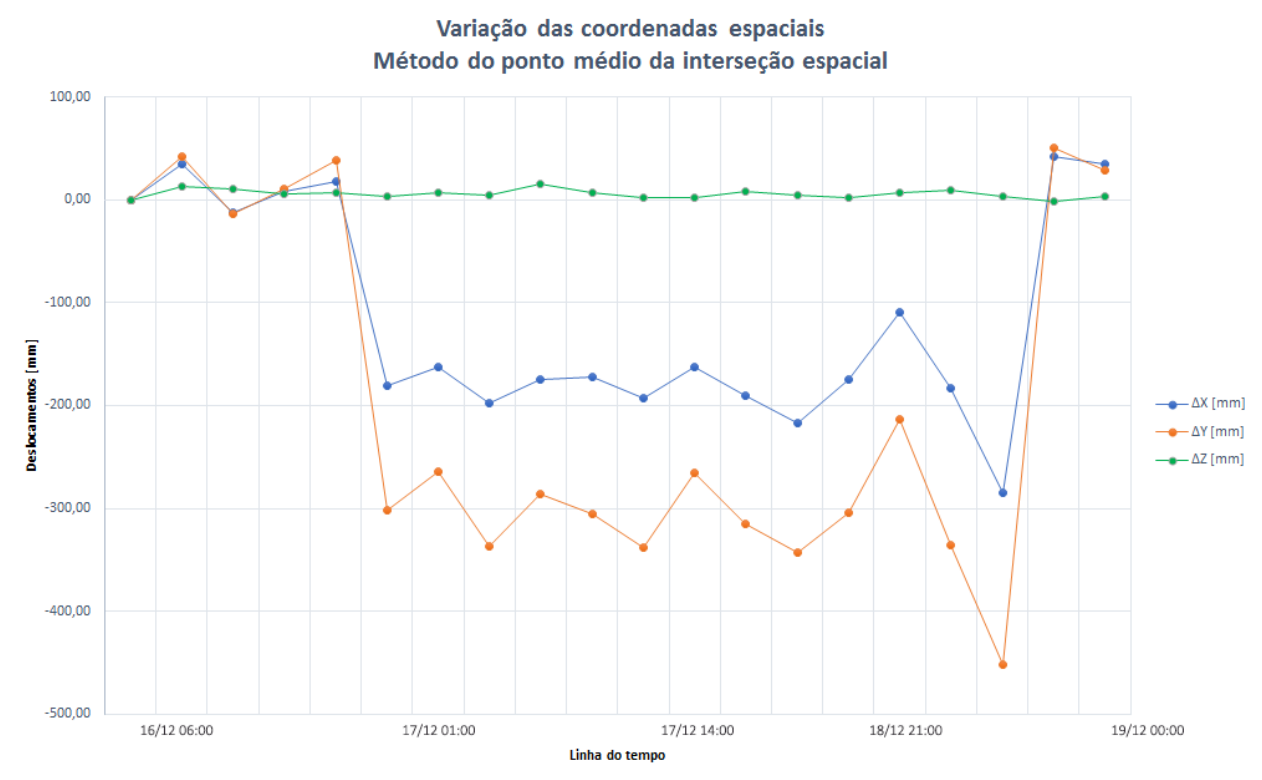

Figura 9.11: Variação das coordenadas espaciais do método do ponto médio da interseção espacial.

A Figura 9.12 apresenta a determinação das coordenadas pelo método do ponto médio da interseção espacial. Dessa forma, é possível visualizar, graficamente, as posições separadamente de $X_{P}$ na Figura 9.12a, de $Y_{P}$ na Figura 9.12b e de $Z_{P}$ na Figura 9.12c.

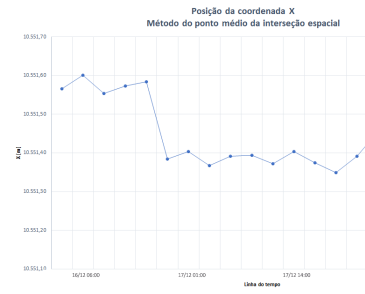

(a) Coordenada $X_{P}$.

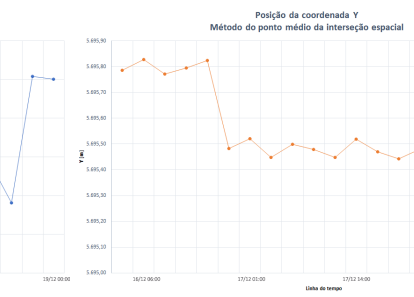

(b) Coordenada $Y_{P}$.

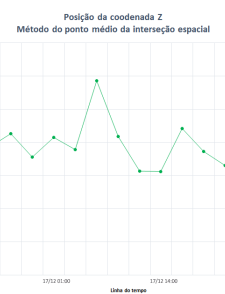

(c) Coordenada $Z_{P}$.

Figura 9.12: Posições das coordenadas $X_{P}, Y_{P}$ e $Z_{P}$ (ponto médio da interseção espacial). 
Os resultados obtidos pelo método das distâncias mínimas com os dados medidos das três estações totais robóticas, podem ser vistos na Tabela 9.13.

Tabela 9.13: Coordenadas espaciais determinadas pelo método das distâncias mínimas.

\begin{tabular}{|c|c|c|c|c|c|c|c|}
\hline \multirow{2}{*}{$\frac{\text { Tempo }}{[\text { Data/Hora }]}$} & \multicolumn{3}{|c|}{ Coordenadas do alvo } & \multicolumn{4}{|c|}{ Precisões } \\
\hline & $X[m]$ & $Y[m]$ & $Z[m]$ & $\sigma_{X}[\mathbf{m m}]$ & $\sigma_{Y}[\mathbf{m m}]$ & $\sigma_{Z}[\mathbf{m m}]$ & $\sigma_{X Y Z}[\mathbf{m m}]$ \\
\hline 16/12/2018 14:51:09 & $10.551,4994$ & $5.695,6952$ & 297,5140 & 7,52990 & 4,94729 & 2,83825 & 9,44621 \\
\hline 16/12/2018 14:56:12 & $10.551,5092$ & $5.695,7036$ & 297,5266 & 7,53027 & 4,94653 & 2,83502 & 9,44513 \\
\hline 16/12/2018 15:01:11 & $10.551,5003$ & $5.695,6989$ & 297,5247 & 7,52976 & 4,94725 & 2,83384 & 9,44474 \\
\hline 16/12/2018 15:06:12 & $10.551,5021$ & $5.695,6984$ & 297,5195 & 7,53004 & 4,94739 & 2,83276 & 9,44472 \\
\hline 16/12/2018 15:11:11 & $10.551,4911$ & $5.695,6978$ & 297,5216 & 7,53018 & 4,94853 & 2,83385 & 9,44576 \\
\hline 17/12/2018 01:01:07 & $10.551,5557$ & $5.695,7066$ & 297,5176 & 7,52997 & 4,94736 & 2,83466 & 9,44522 \\
\hline 17/12/2018 01:06:06 & $10.551,4968$ & $5.695,7024$ & 297,5159 & 7,53029 & & & 9,44527 \\
\hline 17/12/2018 01:11:07 & $10.551,5114$ & $5.695,6895$ & 297,5174 & 7,52995 & 4,94729 & 2,83464 & 9,44516 \\
\hline 17/12/2018 01:16:08 & $10.551,5553$ & $5.695,6916$ & 297,5255 & 7,53022 & 4,94590 & 2,83272 & 9,44407 \\
\hline 17/12/2018 01:21:07 & $10.551,5456$ & $5.695,7119$ & 297,5155 & & & 2,83385 & 9,44477 \\
\hline 17/12/2018 06:46:10 & $10.551,5512$ & $5.695,6887$ & 297,5152 & 7,52955 & 4,94698 & 2,83490 & 9,44475 \\
\hline 17/12/2018 06:51:08 & $10.551,5395$ & $5.695,7038$ & 297,5152 & 7,52976 & 4,94750 & 2,83377 & 9,44486 \\
\hline 17/12/2018 06:56:10 & $10.551,5460$ & $5.695,7014$ & 297,5216 & 7,52976 & 4,94717 & 2,83379 & 9,44469 \\
\hline 17/12/2018 07:01:14 & $10.551,5452$ & $5.695,7081$ & 297,5181 & 7,53040 & 4,94717 & 2,83291 & 9,44494 \\
\hline 17/12/2018 07:06:10 & $10.551,5547$ & $5.695,7014$ & 297,5160 & 7,52995 & 4,94745 & 2,83010 & 9,44388 \\
\hline 18/12/2018 21:01:06 & $10.551,5443$ & $5.695,6902$ & 297,5202 & 7,52976 & 4,94745 & 2,83424 & 9,44497 \\
\hline 18/12/2018 21:06:08 & $10.551,5606$ & $5.695,6904$ & 297,5234 & 7,52965 & 4,94565 & 2,82932 & 9,44246 \\
\hline 18/12/2018 21:11:06 & $10.551,5626$ & $5.695,7131$ & 297,5169 & 7,53052 & 4,94565 & 2,83291 & 9,44423 \\
\hline 18/12/2018 21:16:07 & $10.551,5054$ & $5.695,6972$ & 297,5123 & 7,53052 & 4,94763 & 2,79655 & 9,43443 \\
\hline 18/12/2018 21:21:09 & $10.551,5045$ & $5.695,6838$ & 297,5179 & 7,52959 & 4,94405 & 2,83333 & 9,44277 \\
\hline
\end{tabular}

A Figura 9.13 mostra a visualização gráfica da variação das coordenadas espaciais detectadas pelo método das distâncias mínimas.

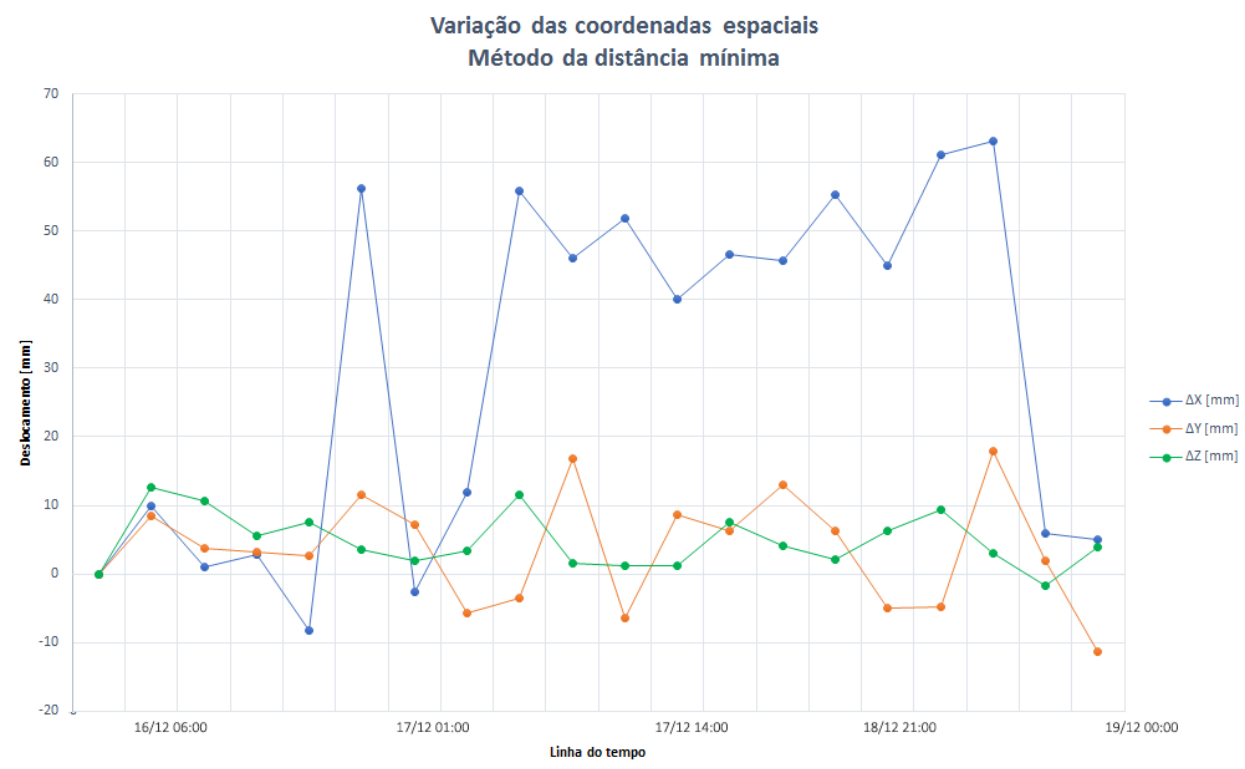

Figura 9.13: Variação das coordenadas espaciais do método das distâncias mínimas. 
A Figura 9.14 apresenta a determinação das coordenadas pelo método das distâncias mínimas. Dessa forma, é possível visualizar, graficamente, as posições separadamente de $X_{P}$ na Figura 9.14a, de $Y_{P}$ na Figura 9.14b e de $Z_{P}$ na Figura 9.14c.

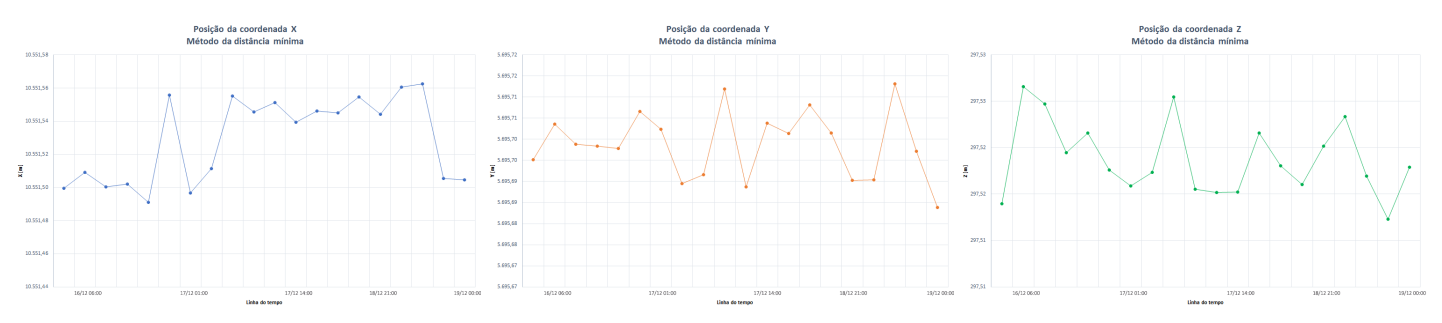
(a) Coordenada $X_{P}$.
(b) Coordenada $Y_{P}$.
(c) Coordenada $Z_{P}$.

Figura 9.14: Posições das coordenadas $X_{P}, Y_{P}$ e $Z_{P}$ (distâncias mínimas).

Os resultados obtidos pelo método das distâncias mínimas com ajuste das observações e, com os dados medidos das três estações totais robóticas, podem ser vistos na Tabela 9.14.

Tabela 9.14: Coordenadas espaciais determinadas pelo método das distâncias mínimas com ajuste das observações.

\begin{tabular}{|c|c|c|c|c|c|c|c|}
\hline \multirow{2}{*}{$\begin{array}{c}\text { Tempo } \\
{[\text { Data/Hora }]}\end{array}$} & \multicolumn{3}{|c|}{ Coordenadas do alvo } & \multicolumn{4}{|c|}{ Precisões } \\
\hline & $X[m]$ & $Y[m]$ & $Z[\mathrm{~m}]$ & $\sigma_{X}[\mathbf{m m}]$ & $\sigma_{Y}[\mathbf{m m}]$ & $\sigma_{Z}[\mathbf{m m}]$ & $\sigma_{X Y Z}[\mathbf{m m}]$ \\
\hline 16/12/2018 14:51:09 & $10.551,4994$ & $5.695,6952$ & 297,5140 & 0,71628 & 0,54938 & 0,14443 & 0,91418 \\
\hline $16 / 12 / 2018$ 14:56:12 & $10.551,5092$ & $5.695,7036$ & 297,5266 & 1,05748 & 0,81108 & 0,21323 & 1,34966 \\
\hline 16/12/2018 15:01:11 & $10.551,5003$ & $5.695,6989$ & 297,5247 & 0,22452 & 0,17221 & 0,04527 & 0,28656 \\
\hline 16/12/2018 15:06:12 & $10.551,5021$ & $5.695,6984$ & 297,5195 & 0,65351 & 0,50126 & 0,13178 & 0,83409 \\
\hline 16/12/2018 15:11:11 & $10.551,4911$ & $5.695,6978$ & 297,5216 & 0,89733 & 0,68829 & 0,18095 & 1,14529 \\
\hline 17/12/2018 01:01:07 & $10.551,5557$ & $5.695,7066$ & 297,5176 & 2,53031 & 1,94061 & 0,51019 & 3,22935 \\
\hline 17/12/2018 01:06:06 & $10.551,4968$ & $5.695,7024$ & 297,5159 & 0,58657 & 0,44993 & 0,11829 & 0,74866 \\
\hline 17/12/2018 01:11:07 & $10.551,5114$ & $5.695,6895$ & 297,5174 & 0,61011 & 0,46794 & 0,12303 & 0,77868 \\
\hline 17/12/2018 01:16:08 & $10.551,5553$ & $5.695,6916$ & 297,5255 & 2,51463 & 1,92849 & 0,50702 & 3,20929 \\
\hline 17/12/2018 01:21:07 & $10.551,5456$ & $5.695,7119$ & 297,5155 & 1,37443 & 1,05406 & 0,27710 & 1,75410 \\
\hline 17/12/2018 06:46:10 & $10.551,5512$ & $5.695,6887$ & 297,5152 & 2,34323 & 1,79691 & 0,47243 & 2,99045 \\
\hline 17/12/2018 06:51:08 & $10.551,5395$ & $5.695,7038$ & 297,5152 & 1,42332 & 1,09154 & 0,28696 & 1,81650 \\
\hline 17/12/2018 06:56:10 & $10.551,5460$ & $5.695,7014$ & 297,5216 & 2,15243 & 1,65076 & 0,43399 & 2,74706 \\
\hline 17/12/2018 07:01:14 & $10.551,5452$ & $5.695,7081$ & 297,5181 & 2,95936 & 2,26967 & 0,59669 & 3,77694 \\
\hline 17/12/2018 07:06:10 & $10.551,5547$ & $5.695,7014$ & 297,5160 & 2,18320 & 1,67426 & 0,44016 & 2,78626 \\
\hline 18/12/2018 21:01:06 & $10.551,5443$ & $5.695,6902$ & 297,5202 & 0,92978 & 0,71307 & 0,18747 & 1,18663 \\
\hline 18/12/2018 21:06:08 & $10.551,5606$ & $5.695,6904$ & 297,5234 & 2,33404 & 1,79002 & 0,47062 & 2,97882 \\
\hline 18/12/2018 21:11:06 & $10.551,5626$ & $5.695,7131$ & 297,5169 & 5,82674 & 4,46903 & 1,17487 & 7,43663 \\
\hline 18/12/2018 21:16:07 & $10.551,5054$ & $5.695,6972$ & 297,5123 & 0,90080 & 0,69092 & 0,18165 & 1,14969 \\
\hline 18/12/2018 21:21:09 & $10.551,5045$ & $5.695,6838$ & 297,5179 & 0,74055 & 0,56799 & 0,14934 & 0,94516 \\
\hline
\end{tabular}


A Figura 9.15 mostra a visualização gráfica da variação das coordenadas espaciais detectadas pelo método das distâncias mínimas com ajustamento.

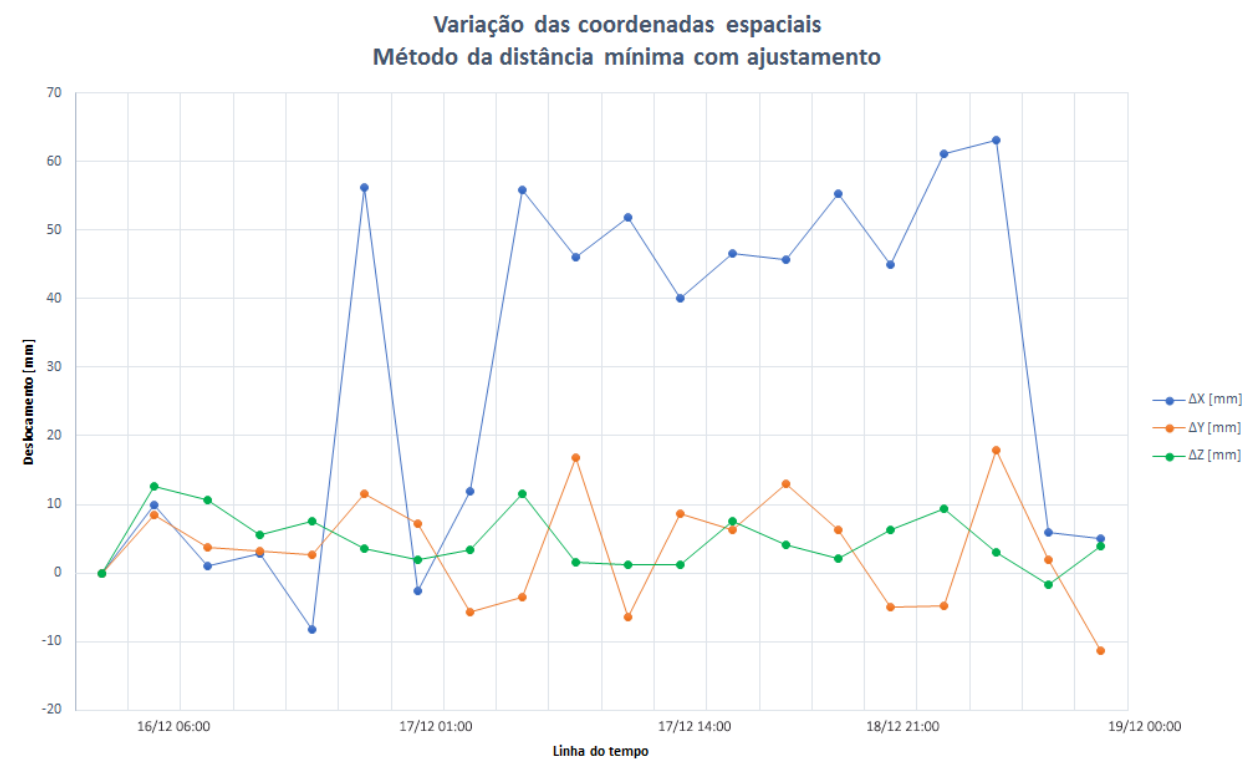

Figura 9.15: Variação das coordenadas espaciais do método das distâncias mínimas com ajustamento.

A Figura 9.16 apresenta a determinação das coordenadas pelo método das distâncias mínimas com ajustamento. Dessa forma, é possível visualizar, graficamente, as posições separadamente de $X_{P}$ na Figura 9.16a, de $Y_{P}$ na Figura 9.16b e de $Z_{P}$ na Figura 9.16c.

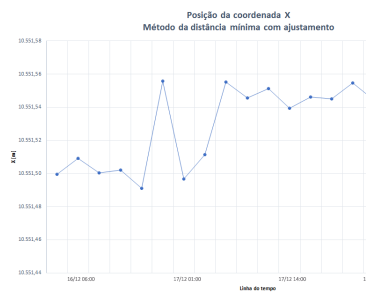

(a) Coordenada $X_{P}$.

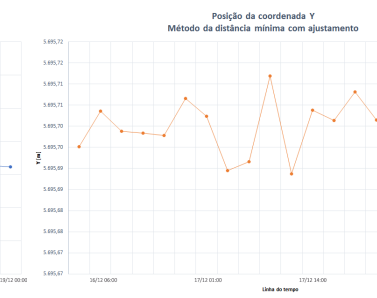

(b) Coordenada $Y_{P}$.

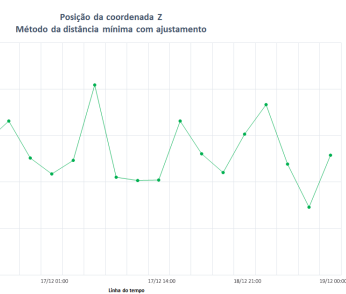

(c) Coordenada $Z_{P}$.

Figura 9.16: Posições das coordenadas $X_{P}, Y_{P}$ e $Z_{P}$ (distâncias mínimas com ajustamento). 
Os resultados obtidos pela média simples entre as coordenadas obtidas com as medições das três estações totais robóticas, tomadas duas a duas, e determinadas pelo método de interseção espacial analítica, podem ser vistos na Tabela 9.15.

Tabela 9.15: Coordenadas espaciais determinadas pelo método de interseção espacial analítica.

\begin{tabular}{|c|c|c|c|}
\hline \multirow{2}{*}{$\frac{\text { Tempo }}{[\text { Data/Hora }]}$} & \multicolumn{3}{|c|}{ Coordenadas do alvo } \\
\hline & $X[m]$ & $Y[m]$ & $Z[m]$ \\
\hline 16/12/2018 14:51:09 & $10.551,5659$ & $5.695,7851$ & 297,5136 \\
\hline 16/12/2018 14:56:12 & $10.551,6005$ & $5.695,8271$ & 297,5261 \\
\hline 16/12/2018 15:01:11 & $10.551,5538$ & $5.695,7711$ & 297,5244 \\
\hline 16/12/2018 15:06:12 & $10.551,5737$ & $5.695,7952$ & 297,5191 \\
\hline 16/12/2018 15:11:11 & $10.551,5840$ & $5.695,8235$ & 297,5211 \\
\hline 17/12/2018 01:01:07 & $10.551,3759$ & $5.695,4635$ & 297,5186 \\
\hline 17/12/2018 01:06:06 & $10.551,5854$ & $5.695,8222$ & 297,5154 \\
\hline 17/12/2018 01:11:07 & $10.551,5991$ & $5.695,8082$ & 297,5169 \\
\hline 17/12/2018 01:16:08 & $10.551,3806$ & $5.695,4554$ & 297,5264 \\
\hline 17/12/2018 01:21:07 & $10.551,4203$ & $5.695,5426$ & 297,5162 \\
\hline 17/12/2018 06:46:10 & $10.551,3726$ & $5.695,4472$ & 297,5162 \\
\hline 17/12/2018 06:51:08 & $10.551,4029$ & $5.695,5191$ & 297,5159 \\
\hline 17/12/2018 06:56:10 & $10.551,3749$ & $5.695,4700$ & 297,5225 \\
\hline 17/12/2018 07:01:14 & $10.551,3488$ & $5.695,4427$ & 297,5191 \\
\hline 17/12/2018 07:06:10 & $10.551,3917$ & $5.695,4810$ & 297,5169 \\
\hline 18/12/2018 21:01:06 & $10.551,4566$ & $5.695,5716$ & 297,5207 \\
\hline 18/12/2018 21:06:08 & $10.551,3825$ & $5.695,4494$ & 297,5243 \\
\hline 18/12/2018 21:11:06 & $10.551,2813$ & $5.695,3327$ & 297,5185 \\
\hline 18/12/2018 21:16:07 & $10.551,6083$ & $5.695,8363$ & 297,5117 \\
\hline 18/12/2018 21:21:09 & $10.551,6006$ & $5.695,8137$ & 297,5174 \\
\hline
\end{tabular}


A Figura 9.17 mostra a visualização gráfica da variação das coordenadas espaciais detectadas pelo método de interseção espacial analítica.

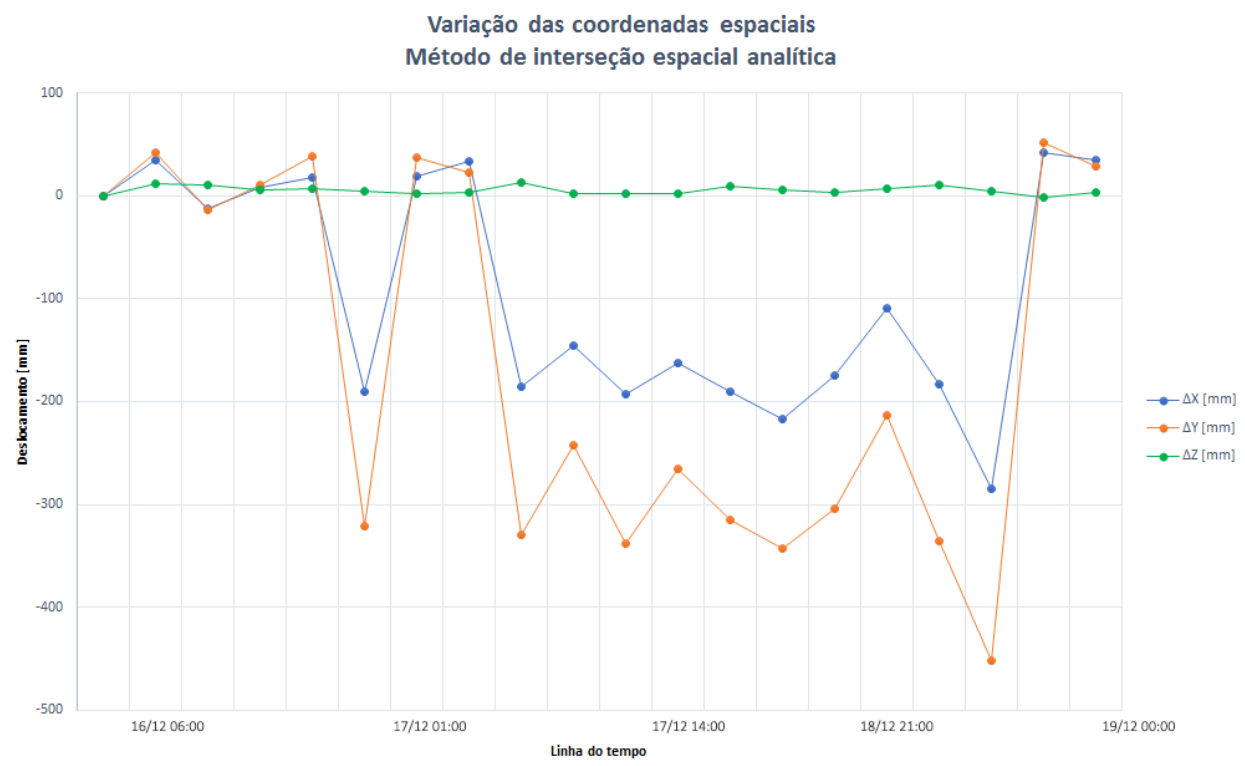

Figura 9.17: Variação das coordenadas espaciais do método de interseção espacial analítica.

A Figura 9.18 apresenta a determinação das coordenadas pelo método de interseção espacial analítica. Dessa forma, é possível visualizar, graficamente, as posições separadamente de $X_{P}$ na Figura 9.18a, de $Y_{P}$ na Figura 9.18b e de $Z_{P}$ na Figura 9.18c.

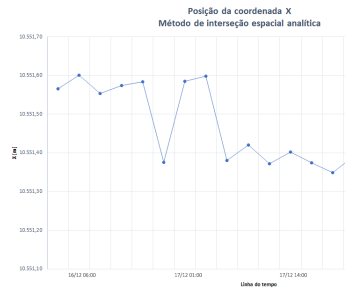

(a) Coordenada $X_{P}$.

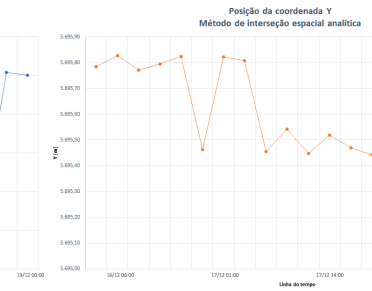

(b) Coordenada $Y_{P}$.

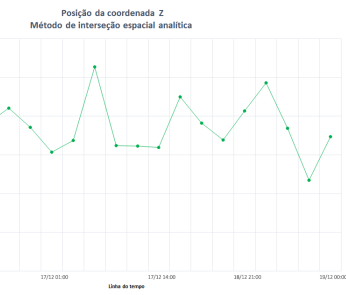

(c) Coordenada $Z_{P}$.

Figura 9.18: Posições das coordenadas $X_{P}, Y_{P}$ e $Z_{P}$ (interseção espacial analítica). 
Os resultados obtidos pelo método da minimização dos deslocamentos em função dos azimutes, dos ângulos verticais e das distâncias inclinadas medidas (relação geométrica), com os dados medidos das três estações totais robóticas, podem ser vistos na Tabela 9.16.

Tabela 9.16: Coordenadas espaciais determinadas pelo método da minimização dos deslocamentos em função dos azimutes, dos ângulos verticais e das distâncias inclinadas medidas (relação geométrica).

\begin{tabular}{|c|c|c|c|c|c|c|c|}
\hline \multirow{2}{*}{$\begin{array}{c}\text { Tempo } \\
{[\text { Data/Hora }]}\end{array}$} & \multicolumn{3}{|c|}{ Coordenadas do alvo } & \multicolumn{4}{|c|}{ Precisões } \\
\hline & $X[m]$ & $Y[m]$ & $Z[\mathrm{~m}]$ & $\sigma_{X}[\mathbf{m m}]$ & $\sigma_{Y}[\mathbf{m m}]$ & $\sigma_{Z}[\mathbf{m m}]$ & $\sigma_{X Y Z}[\mathbf{m m}]$ \\
\hline 16/12/2018 14:51:09 & $10.551,5117$ & $5.695,7184$ & 297,5140 & 2,59838 & 2,61191 & 0,92080 & 3,79757 \\
\hline 16/12/2018 14:56:12 & $10.551,5131$ & $5.695,7184$ & 297,5267 & 1,83289 & 1,84229 & 0,64950 & 2,67869 \\
\hline 16/12/2018 15:01:11 & $10.551,5183$ & $5.695,7174$ & 297,5245 & 1,41527 & 1,42359 & 0,50154 & 2,06909 \\
\hline 16/12/2018 15:06:12 & $10.551,5167$ & $5.695,7179$ & 297,5194 & 0,74094 & 0,74523 & 2,62591 & 2,82839 \\
\hline 16/12/2018 15:11:11 & $10.551,5039$ & $5.695,7168$ & 297,5215 & 0,60073 & 0,60414 & 2,12896 & 2,29311 \\
\hline 17/12/2018 01:01:07 & $10.551,5411$ & $5.695,7196$ & 297,5179 & 0,65087 & 0,65491 & 2,30652 & 2,48447 \\
\hline 17/12/2018 01:06:06 & $10.551,5387$ & $5.695,7202$ & 297,5207 & 1,04692 & 1,05344 & 3,71000 & 3,99623 \\
\hline 17/12/2018 01:11:07 & $10.551,5413$ & $5.695,6993$ & 297,5188 & 0,74105 & 0,74561 & 2,62628 & 2,82886 \\
\hline 17/12/2018 01:16:08 & $10.551,5353$ & $5.695,7102$ & 297,5293 & 2,17883 & 2,19242 & 0,77210 & 3,18592 \\
\hline 17/12/2018 01:21:07 & $10.551,5531$ & $5.695,7125$ & 297,5208 & 3,78950 & 3,81361 & 1,34291 & 5,54142 \\
\hline 17/12/2018 06:46:10 & $10.551,5395$ & $5.695,6936$ & 297,5156 & & & 3,96083 & 4,26631 \\
\hline 17/12/2018 06:51:08 & $10.551,5360$ & $5.695,7134$ & 297,5155 & 1,77746 & 1,78808 & 0,62989 & 2,59872 \\
\hline 17/12/2018 06:56:10 & $10.551,5249$ & $5.695,6984$ & 297,5222 & 3,74285 & 3,76541 & 1,32634 & 5,47233 \\
\hline 17/12/2018 07:01:14 & $10.551,5304$ & $5.695,7051$ & 297,5184 & 2,07455 & 2,08729 & 0,73523 & 3,03333 \\
\hline 17/12/2018 07:06:10 & $10.551,5404$ & $5.695,7019$ & 297,5165 & 0,50614 & 0,50928 & 1,79389 & 1,93225 \\
\hline 18/12/2018 21:01:06 & $10.551,5331$ & $5.695,6888$ & 297,5205 & 0,58171 & 0,58528 & 2,06144 & 2,22047 \\
\hline 18/12/2018 21:06:08 & $10.551,5299$ & $5.695,6729$ & 297,5239 & 3,41614 & 3,43614 & 1,21059 & 4,99425 \\
\hline 18/12/2018 21:11:06 & $10.551,5180$ & $5.695,6808$ & 297,5176 & 1,00289 & 1,00886 & 3,55421 & 3,82831 \\
\hline 18/12/2018 21:16:07 & $10.551,5189$ & $5.695,7131$ & 297,5122 & 0,77126 & 0,77572 & 2,73325 & 2,94402 \\
\hline 18/12/2018 21:21:09 & $10.551,5199$ & $5.695,7009$ & 297,5177 & 0,84703 & 0,85181 & 3,00172 & 3,23317 \\
\hline
\end{tabular}


A Figura 9.19 mostra a visualização gráfica da variação das coordenadas espaciais detectadas pelo método da minimização dos deslocamentos em função dos azimutes, dos ângulos verticais e das distâncias inclinadas medidas (relação geométrica).

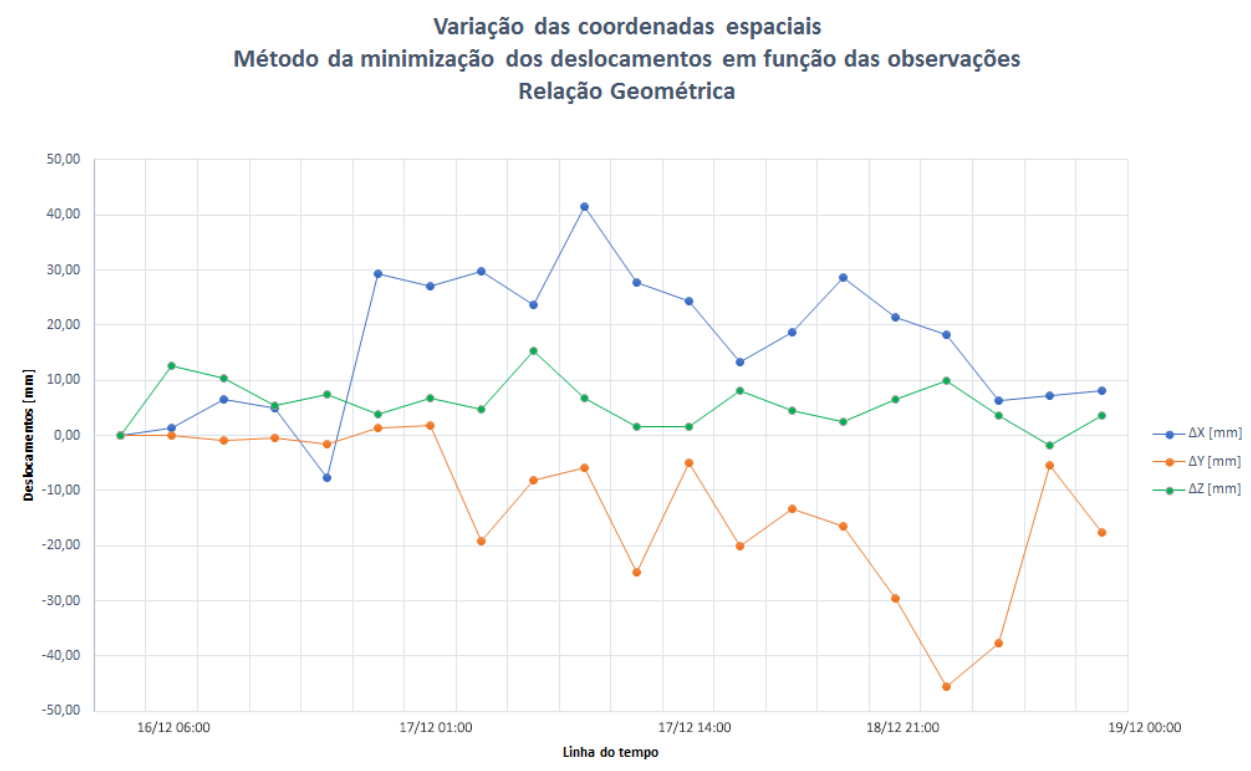

Figura 9.19: Variação das coordenadas espaciais do método da minimização dos deslocamentos em função dos azimutes, dos ângulos verticais e das distâncias inclinadas medidas (relação geométrica).

A Figura 9.20 apresenta a determinação das coordenadas método da minimização dos deslocamentos em função dos azimutes, dos ângulos verticais e das distâncias inclinadas medidas (relação geométrica). Dessa forma, é possível visualizar, graficamente, as posições separadamente de $X_{P}$ na Figura 9.20a, de $Y_{P}$ na Figura 9.20b e de $Z_{P}$ na Figura 9.20c.

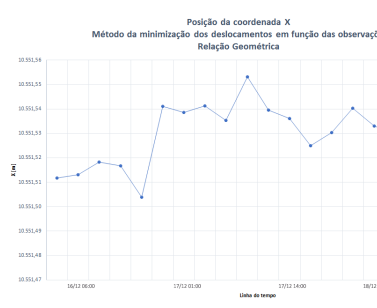

(a) Coordenada $X_{P}$.

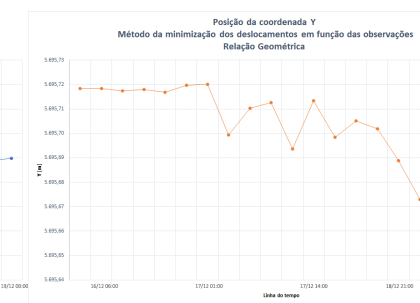

(b) Coordenada $Y_{P}$.

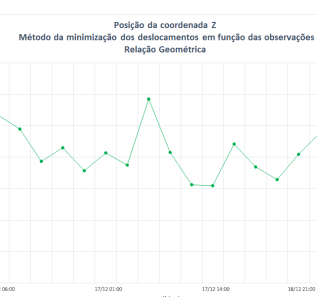

(c) Coordenada $Z_{P}$.

Figura 9.20: Posições das coordenadas $X_{P}, Y_{P}$ e $Z_{P}$ (minimização com relação geométrica). 
Os resultados obtidos pelo método da minimização dos resíduos das funções trigonométricas em função dos azimutes, dos ângulos verticais e distâncias inclinadas medidas (relação analítica), com os dados medidos das três estações totais robóticas, podem ser vistos na Tabela 9.17.

Tabela 9.17: Coordenadas espaciais determinadas pelo método da minimização dos resíduos das funções trigonométricas em função dos azimutes, dos ângulos verticais e distâncias inclinadas medidas (relação analítica).

\begin{tabular}{|c|c|c|c|c|c|c|c|}
\hline \multirow{2}{*}{$\begin{array}{c}\text { Tempo } \\
{[\text { Data/Hora }]}\end{array}$} & \multicolumn{3}{|c|}{ Coordenadas do alvo } & \multicolumn{4}{|c|}{ Precisões } \\
\hline & $X[m]$ & $Y[m]$ & $Z[\mathrm{~m}]$ & $\sigma_{X}[\mathbf{m m}]$ & $\sigma_{Y}[\mathbf{m m}]$ & $\sigma_{Z}[\mathbf{m m}]$ & $\sigma_{X Y Z}[\mathbf{m m}]$ \\
\hline 16/12/2018 14:51:09 & $10.551,5117$ & $5.695,7184$ & 297,5140 & 1,32819 & 0,44629 & 0,83568 & 1,63145 \\
\hline 16/12/2018 14:56:12 & $10.551,5131$ & $5.695,7184$ & 297,5267 & 0,91707 & 3,11814 & 0,57735 & 3,30109 \\
\hline 16/12/2018 15:01:11 & $10.551,5183$ & $5.695,7174$ & 297,5245 & 0,74147 & 2,51613 & 0,46524 & 2,66404 \\
\hline 16/12/2018 15:06:12 & $10.551,5167$ & $5.695,7179$ & 297,5194 & 0,53881 & 1,80268 & 3,38218 & 3,87029 \\
\hline 16/12/2018 15:11:11 & $10.551,5039$ & $5.695,7168$ & 297,5215 & 1,06574 & 0,35796 & 0,66891 & 1,30819 \\
\hline 17/12/2018 01:01:07 & $10.551,5411$ & $5.695,7196$ & 297,5179 & 1,82686 & 0,62821 & 1,14614 & 2,24626 \\
\hline 17/12/2018 01:06:06 & $10.551,5387$ & $5.695,7202$ & 297,5207 & 3,28628 & 1,13159 & 2,06322 & 4,04191 \\
\hline 17/12/2018 01:11:07 & $10.551,5413$ & $5.695,6993$ & 297,5188 & 1,57108 & 0,54438 & 0,98932 & 1,93479 \\
\hline 17/12/2018 01:16:08 & $10.551,5353$ & $5.695,7102$ & 297,5293 & 1,92913 & 0,67288 & 1,21205 & 2,37558 \\
\hline 17/12/2018 01:21:07 & $10.551,5531$ & $5.695,7125$ & 297,5208 & 0,36935 & 1,28044 & 2,31707 & 2,67296 \\
\hline 17/12/2018 06:46:10 & $10.551,5395$ & $5.695,6936$ & 297,5156 & 1,79779 & 0,61509 & 1,12940 & 2,21041 \\
\hline 17/12/2018 06:51:08 & $10.551,5360$ & $5.695,7134$ & 297,5155 & 0,42160 & 1,44020 & 2,64638 & 3,04224 \\
\hline 17/12/2018 06:56:10 & $10.551,5249$ & $5.695,6984$ & 297,5222 & 0,46253 & 1,58883 & 2,90467 & 3,34296 \\
\hline 17/12/2018 07:01:14 & $10.551,5304$ & $5.695,7051$ & 297,5184 & 3,32647 & 1,14521 & 2,09498 & 4,09461 \\
\hline 17/12/2018 07:06:10 & $10.551,5404$ & $5.695,7019$ & 297,5165 & 2,56127 & 0,88092 & 1,61341 & 3,15266 \\
\hline 18/12/2018 21:01:06 & $10.551,5331$ & $5.695,6888$ & 297,5205 & 0,58084 & 1,97529 & 3,63621 & 4,17865 \\
\hline 18/12/2018 21:06:08 & $10.551,5299$ & $5.695,6729$ & 297,5239 & 3,95790 & 1,35553 & 2,48317 & 4,86504 \\
\hline 18/12/2018 21:11:06 & $10.551,5180$ & $5.695,6808$ & 297,5176 & 0,92117 & 0,31491 & 0,57911 & 1,13273 \\
\hline 18/12/2018 21:16:07 & $10.551,5189$ & $5.695,7131$ & 297,5122 & 2,51257 & 0,84858 & 1,57487 & 3,08437 \\
\hline 18/12/2018 21:21:09 & $10.551,5199$ & $5.695,7009$ & 297,5177 & 1,82026 & 0,61902 & 1,14132 & 2,23587 \\
\hline
\end{tabular}


A Figura 9.21 mostra a visualização gráfica da variação das coordenadas espaciais detectadas pelo método da minimização dos resíduos das funções trigonométricas em função dos azimutes, dos ângulos verticais e distâncias inclinadas medidas (relação analítica).

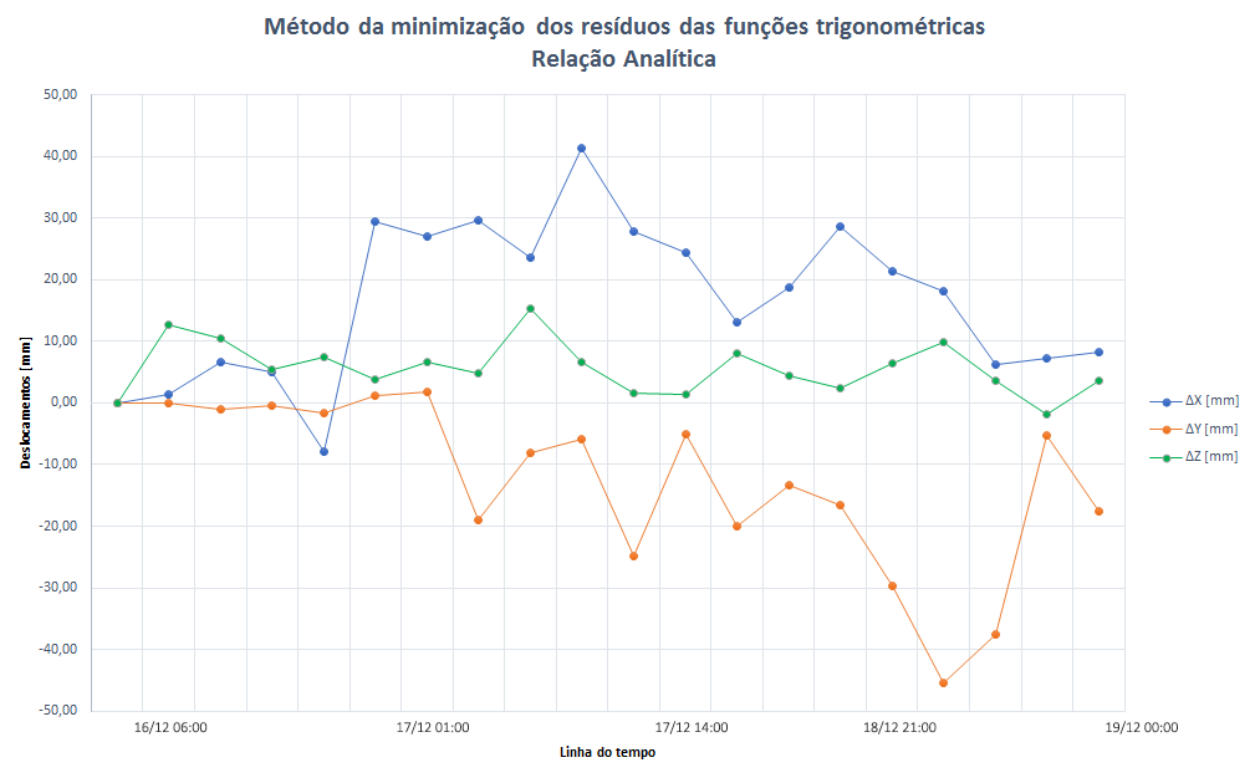

Figura 9.21: Variação das coordenadas espaciais do método da minimização dos resíduos das funções trigonométricas em função dos azimutes, dos ângulos verticais e distâncias inclinadas medidas (relação analítica).

A Figura 9.22 apresenta a determinação das coordenadas método da minimização dos resíduos das funções trigonométricas em função dos azimutes, dos ângulos verticais e distâncias inclinadas medidas (relação analítica). Dessa forma, é possível visualizar, graficamente, as posições separadamente de $X_{P}$ na Figura 9.22a, de $Y_{P}$ na Figura 9.22b e de $Z_{P}$ na Figura 9.22c.

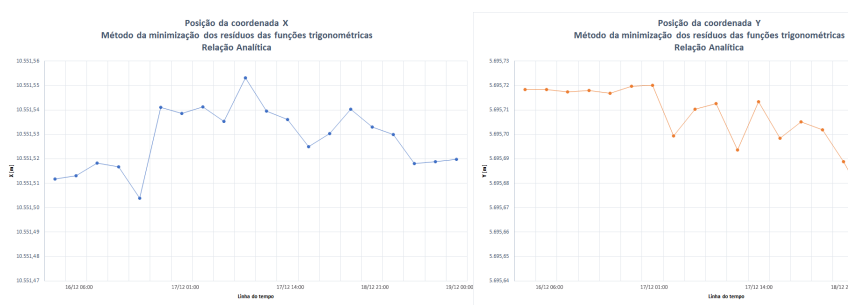

(a) Coordenada $X_{P}$. (b) Coordenada $Y_{P}$.

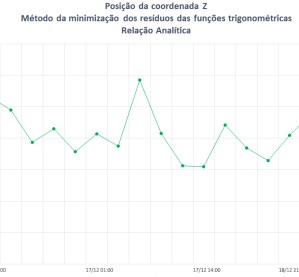

(c) Coordenada $Z_{P}$.

Figura 9.22: Posições das coordenadas $X_{P}, Y_{P}$ e $Z_{P}$ (minimização com relação analítica). 
Os resultados obtidos pela média simples entre as coordenadas obtidas com as medições das três estações totais robóticas, tomadas duas a duas, e determinadas pelo método iterativo de aproximações sucessivas, podem ser vistos na Tabela 9.18.

Tabela 9.18: Coordenadas espaciais determinadas pelo método iterativo de aproximações sucessivas, para determinação de coordenadas espaciais, considerando duas estações totais robóticas.

\begin{tabular}{|c|c|c|c|c|c|c|c|}
\hline \multirow{2}{*}{$\begin{array}{c}\text { Tempo } \\
{[\text { Data/Hora }]}\end{array}$} & \multicolumn{3}{|c|}{ Coordenadas do alvo } & \multicolumn{4}{|c|}{ Precisões } \\
\hline & $X[m]$ & $Y[m]$ & $Z[\mathrm{~m}]$ & $\sigma_{X}[\mathbf{m m}]$ & $\sigma_{Y}[\mathbf{m m}]$ & $\sigma_{Z}[\mathbf{m m}]$ & $\sigma_{X Y Z}[\mathbf{m m}]$ \\
\hline 16/12/2018 14:51:09 & $10.551,5104$ & $5.695,7180$ & 297,5127 & 0,34559 & 1,16528 & 2,17015 & 2,48734 \\
\hline 16/12/2018 14:56:12 & $10.551,5111$ & $5.695,7176$ & 297,5262 & 0,38244 & 1,28611 & 2,40194 & 2,75130 \\
\hline 16/12/2018 15:01:11 & $10.551,5178$ & $5.695,7144$ & 297,5297 & 1,35891 & 0,45817 & 0,85256 & 1,66836 \\
\hline 16/12/2018 15:06:12 & $10.551,5157$ & $5.695,7200$ & 297,5200 & 0,84085 & 2,78984 & 0,52636 & 2,96096 \\
\hline 16/12/2018 15:11:11 & $10.551,5044$ & $5.695,7174$ & 297,5227 & 1,91360 & 0,63652 & 1,19785 & 2,34560 \\
\hline 17/12/2018 01:01:07 & $10.551,5389$ & $5.695,7179$ & 297,5204 & 0,91477 & 0,31672 & 0,57547 & 1,12618 \\
\hline 17/12/2018 01:06:06 & $10.551,5390$ & $5.695,7205$ & 297,5172 & 0,88555 & 0,30604 & 0,55731 & 1,09017 \\
\hline 17/12/2018 01:11:07 & $10.551,5397$ & $5.695,6976$ & 297,5217 & 1,33824 & 0,46280 & 0,84209 & 1,64748 \\
\hline 17/12/2018 01:16:08 & $10.551,5369$ & $5.695,7068$ & 297,5230 & 2,58768 & 0,89735 & 1,62975 & 3,18707 \\
\hline 17/12/2018 01:21:07 & $10.551,5516$ & $5.695,7103$ & 297,5213 & 1,45726 & 0,50817 & 0,91738 & 1,79539 \\
\hline 17/12/2018 06:46:10 & $10.551,5390$ & $5.695,6934$ & 297,5171 & 0,97743 & 0,33563 & 0,61416 & 1,20217 \\
\hline 17/12/2018 06:51:08 & $10.551,5372$ & $5.695,7103$ & 297,5153 & 0,24152 & 0,82980 & 1,51772 & 1,74653 \\
\hline 17/12/2018 06:56:10 & $10.551,5222$ & $5.695,6989$ & 297,5214 & 1,55923 & 0,53454 & 0,97996 & 1,91762 \\
\hline 17/12/2018 07:01:14 & $10.551,5303$ & $5.695,7037$ & 297,5134 & 0,31710 & 1,08592 & 1,99261 & 2,29134 \\
\hline 17/12/2018 07:06:10 & $10.551,5419$ & $5.695,7011$ & 297,5190 & 1,55838 & 0,53312 & 0,97872 & 1,91589 \\
\hline 18/12/2018 21:01:06 & $10.551,5321$ & $5.695,6885$ & 297,5224 & 1,40463 & 0,48055 & 0,88266 & 1,72714 \\
\hline 18/12/2018 21:06:08 & $10.551,5286$ & $5.695,6723$ & 297,5236 & 0,55846 & 1,90833 & 3,50809 & 4,03241 \\
\hline 18/12/2018 21:11:06 & $10.551,5178$ & $5.695,6805$ & 297,5143 & 1,22140 & 0,41662 & 0,76700 & 1,50122 \\
\hline 18/12/2018 21:16:07 & $10.551,5184$ & $5.695,7106$ & 297,5101 & 1,58907 & 0,54216 & 0,99758 & 1,95301 \\
\hline 18/12/2018 21:21:09 & $10.551,5209$ & $5.695,7015$ & 297,5159 & 1,83370 & 0,62645 & 1,15127 & 2,25396 \\
\hline
\end{tabular}


A Figura 9.23 mostra a visualização gráfica da variação das coordenadas espaciais detectadas pelo método iterativo de aproximações sucessivas para determinação de coordenadas espaciais considerando duas estações totais robóticas.

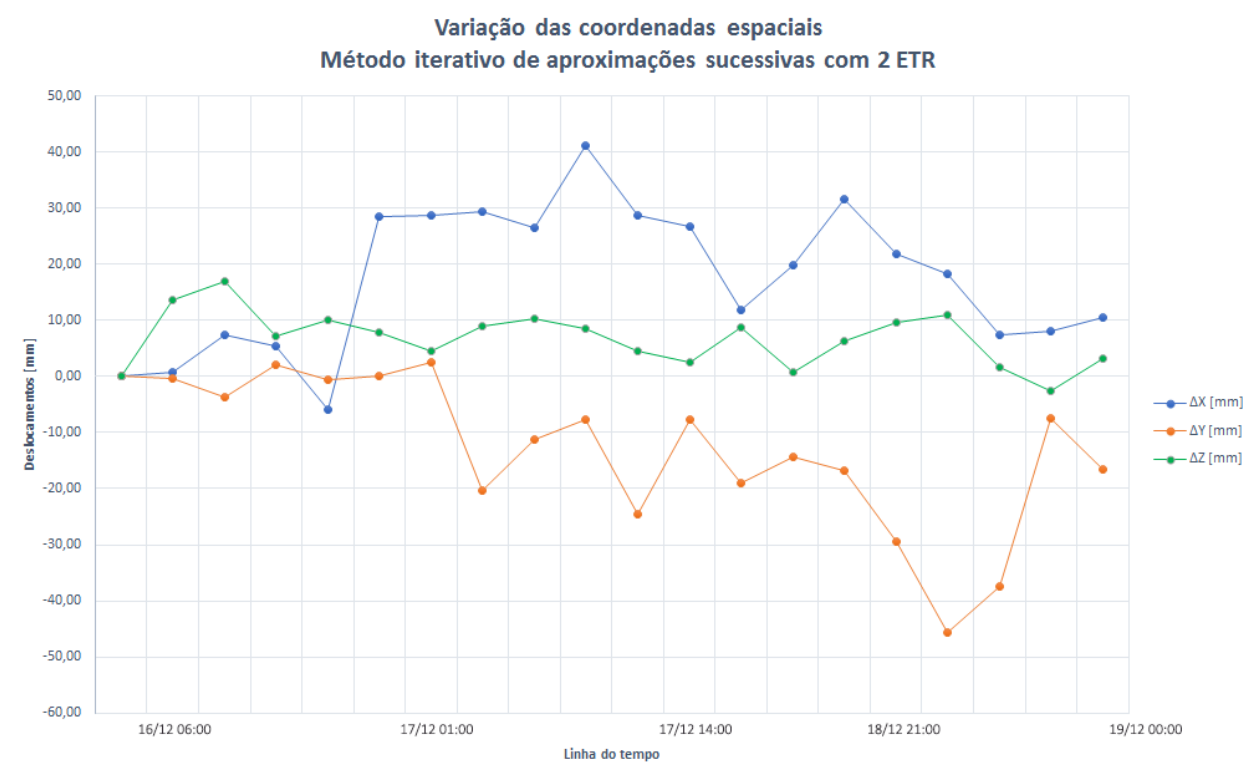

Figura 9.23: Variação das coordenadas espaciais do método iterativo de aproximações sucessivas, para determinação de coordenadas espaciais, considerando duas estações totais robóticas.

A Figura 9.24 apresenta a determinação das coordenadas método iterativo de aproximações sucessivas para determinação de coordenadas espaciais considerando duas estações totais robóticas. Dessa forma, é possível visualizar, graficamente, as posições separadamente de $X_{P}$ na Figura 9.24a, de $Y_{P}$ na Figura 9.24b e de $Z_{P}$ na Figura 9.24c.

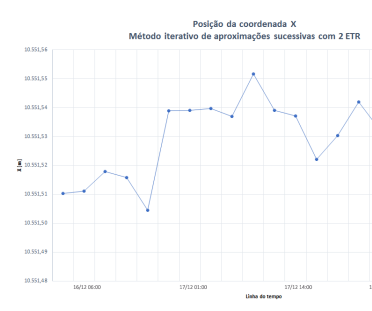

(a) Coordenada $X_{P}$.

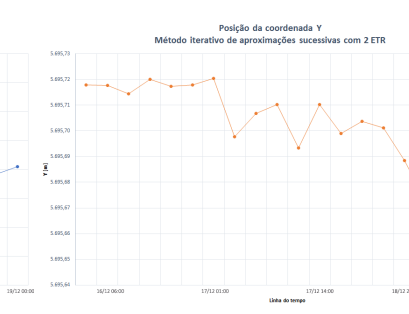

(b) Coordenada $Y_{P}$.

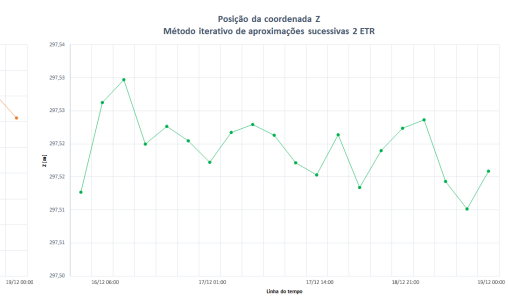

(c) Coordenada $Z_{P}$.

Figura 9.24: Posições das coordenadas $X_{P}, Y_{P}$ e $Z_{P}$ (iterativo com 2 ETR). 
Os resultados obtidos pelo método iterativo de aproximações sucessivas para determinação de coordenadas espaciais considerando três estações totais robóticas, com os dados medidos das três estações totais robóticas, podem ser vistos na Tabela 9.19.

Tabela 9.19: Coordenadas espaciais determinadas pelo método iterativo de aproximações sucessivas, para determinação de coordenadas espaciais, considerando três estações totais robóticas.

\begin{tabular}{|c|c|c|c|c|c|c|c|}
\hline \multirow{2}{*}{$\begin{array}{c}\text { Tempo } \\
{[\text { Data/Hora }]}\end{array}$} & \multicolumn{3}{|c|}{ Coordenadas do alvo } & \multicolumn{4}{|c|}{ Precisões } \\
\hline & $X[m]$ & $Y[m]$ & $Z[m]$ & $\sigma_{X}[\mathbf{m m}]$ & $\sigma_{Y}[\mathbf{m m}]$ & $\sigma_{Z}[\mathbf{m m}]$ & $\sigma_{X Y Z}[\mathbf{m m}]$ \\
\hline 16/12/2018 14:51:09 & $10.551,5128$ & $5.695,7199$ & 297,5150 & 0,15521 & 0,52154 & 0,97541 & 1,11692 \\
\hline 16/12/2018 14:56:12 & $10.551,5134$ & $5.695,7193$ & 297,5290 & 0,15964 & 0,54004 & 1,00223 & 1,14960 \\
\hline 16/12/2018 15:01:11 & $10.551,5199$ & $5.695,7176$ & 297,5335 & 0,34045 & 1,15343 & 0,21354 & 1,22143 \\
\hline 16/12/2018 15:06:12 & $10.551,5178$ & $5.695,7225$ & 297,5238 & 0,73149 & 0,24288 & 0,45803 & 0,89659 \\
\hline 16/12/2018 15:11:11 & $10.551,5075$ & $5.695,7191$ & 297,5257 & 1,08569 & 0,36189 & 0,67968 & 1,33104 \\
\hline 17/12/2018 01:01:07 & $10.551,5408$ & $5.695,7212$ & 297,5232 & 0,23656 & 0,81952 & 1,48855 & 1,71562 \\
\hline 17/12/2018 01:06:06 & $10.551,5418$ & $5.695,7227$ & 297,5205 & 0,21879 & 0,75829 & 1,37685 & 1,58700 \\
\hline 17/12/2018 01:11:07 & $10.551,5412$ & $5.695,7007$ & 297,5255 & 0,64640 & 0,22371 & 0,40686 & 0,79587 \\
\hline 17/12/2018 01:16:08 & $10.551,5393$ & $5.695,7115$ & 297,5268 & 0,97650 & 0,34168 & 0,61449 & 1,20328 \\
\hline 17/12/2018 01:21:07 & $10.551,5530$ & $5.695,7126$ & 297,5246 & 0,30661 & 1,07273 & 1,92995 & 2,22923 \\
\hline 17/12/2018 06:46:10 & $10.551,5424$ & $5.695,6948$ & 297,5184 & & & & 0,89982 \\
\hline 17/12/2018 06:51:08 & $10.551,5394$ & $5.695,7143$ & 297,5192 & 1,04769 & 0,35930 & 0,65875 & 1,28868 \\
\hline 17/12/2018 06:56:10 & $10.551,5263$ & $5.695,7005$ & 297,5235 & 0,94886 & 0,32633 & 0,59632 & 1,16724 \\
\hline 17/12/2018 07:01:14 & $10.551,5330$ & $5.695,7066$ & 297,5171 & 0,50905 & 0,17435 & 0,31999 & 0,62603 \\
\hline 17/12/2018 07:06:10 & $10.551,5438$ & $5.695,7029$ & 297,5232 & 0,72319 & 0,24716 & 0,45442 & 0,88915 \\
\hline 18/12/2018 21:01:06 & $10.551,5350$ & $5.695,6916$ & 297,5252 & 0,23620 & 0,80937 & 0,14845 & 0,85610 \\
\hline 18/12/2018 21:06:08 & $10.551,5307$ & $5.695,6739$ & 297,5273 & 1,18500 & 0,40630 & 0,74437 & 1,45719 \\
\hline 18/12/2018 21:11:06 & $10.551,5207$ & $5.695,6833$ & 297,5170 & 0,23419 & 0,79726 & 1,47161 & 1,69000 \\
\hline 18/12/2018 21:16:07 & $10.551,5202$ & $5.695,7130$ & 297,5121 & 0,33232 & 1,12867 & 0,20882 & 1,19497 \\
\hline 18/12/2018 21:21:09 & $10.551,5216$ & $5.695,7033$ & 297,5193 & 0,24474 & 0,83561 & 0,15373 & 0,88418 \\
\hline
\end{tabular}


A Figura 9.25 mostra a visualização gráfica da variação das coordenadas espaciais detectadas pelo método iterativo de aproximações sucessivas para determinação de coordenadas espaciais considerando três estações totais robóticas.

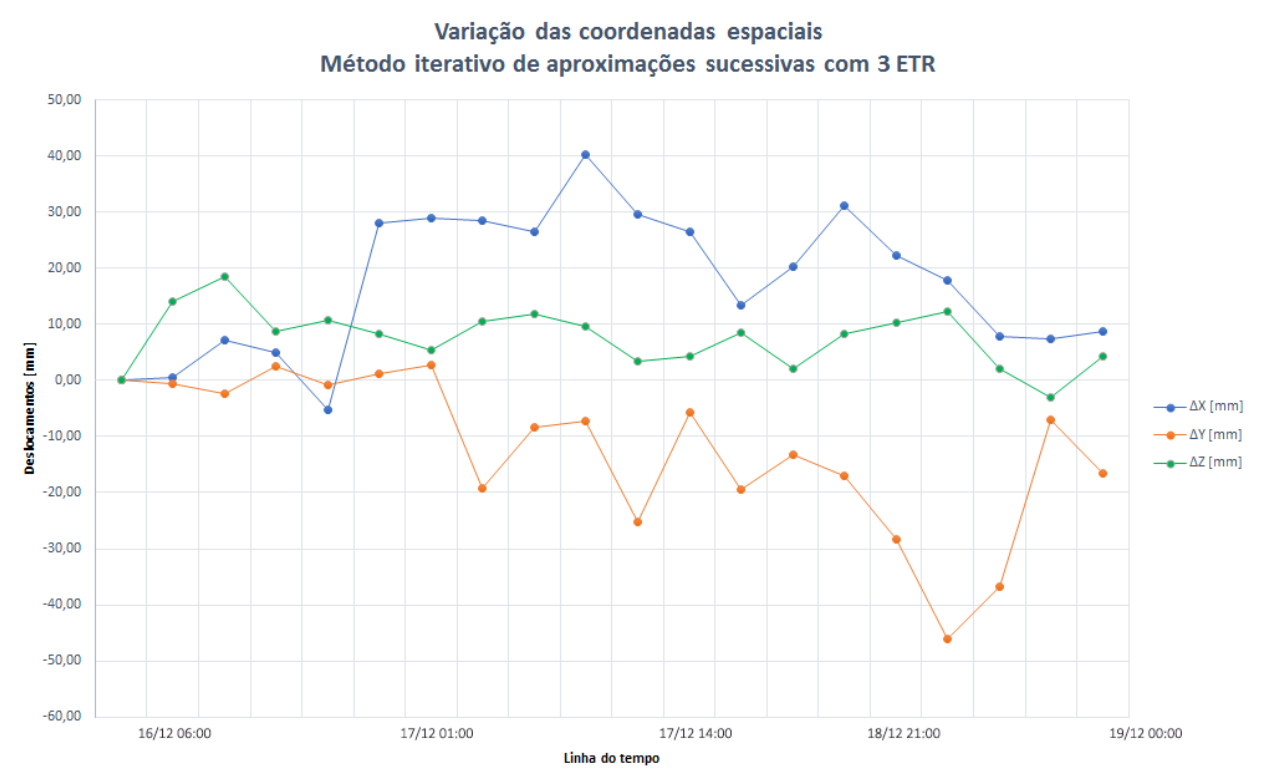

Figura 9.25: Variação das coordenadas espaciais do método iterativo de aproximações sucessivas, para determinação de coordenadas espaciais, considerando três estações totais robóticas.

A Figura 9.26 apresenta a determinação das coordenadas pelo método iterativo de aproximações sucessivas para determinação de coordenadas espaciais considerando três estações totais robóticas. Dessa forma, é possível visualizar, graficamente, as posições separadamente de $X_{P}$ na Figura 9.26a, de $Y_{P}$ na Figura 9.26b e de $Z_{P}$ na Figura 9.26c.

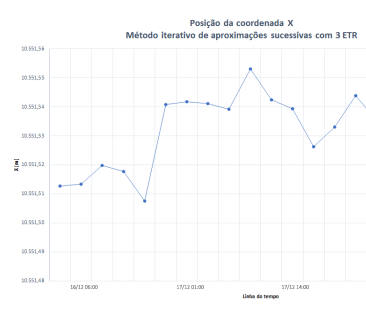

(a) Coordenada $X_{P}$.

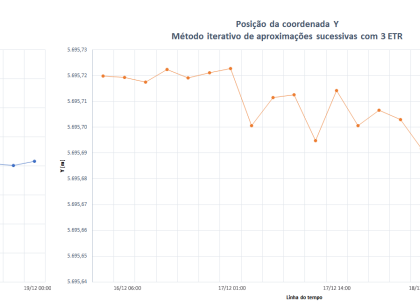

(b) Coordenada $Y_{P}$.

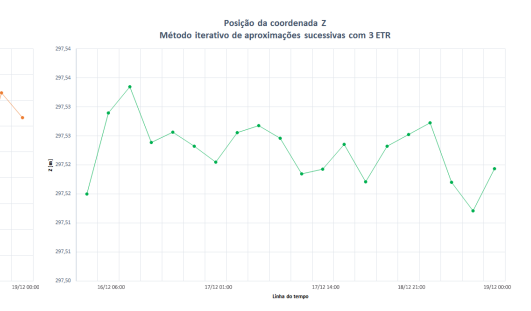

(c) Coordenada $Z_{P}$.

Figura 9.26: Posições das coordenadas $X_{P}, Y_{P}$ e $Z_{P}$ (iterativo com 3 ETR).

Devido a magnitude do banco de dados gerado, foi mostrado detalhadamente, algumas amostras, escolhidas aleatoriamente e agrupadas, para representar as informações deste universo de dados. Porém, com o objetivo de apresentar o monitoramento geodésico de estruturas realizado em sua totalidade, extraiu-se do subsistema de processamento, os gráficos contendo todas as variações que o braço robótico realizou de forma controlada e os gráficos contendo as variações detectadas por cada método iterativo proposto, para que assim, possa verificar o grau de aderência destes métodos propostos em relação ao controle. 
Assim, a Figura 9.27 mostra as movimentações controladas e executadas pelo braço robótico durante todo o experimento de monitoramento. E, para melhor visualização, a Figura 9.27a mostra a movimentação aplicada para a coordenada $X_{P}$, a Figura 9.27b para a coordenada $Y_{P}$ e a Figura $9.27 \mathrm{c}$ para a coordenada $Z_{P}$.

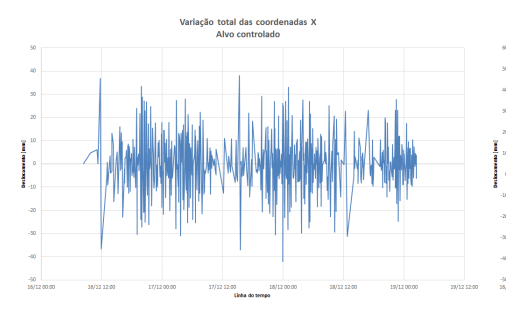

(a) Coordenada $X_{P}$.

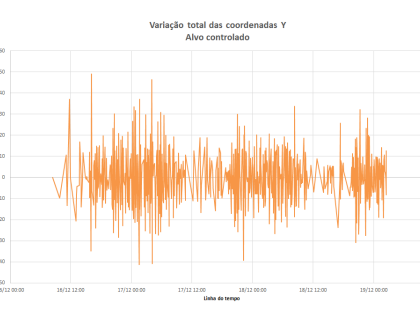

(b) Coordenada $Y_{P}$.

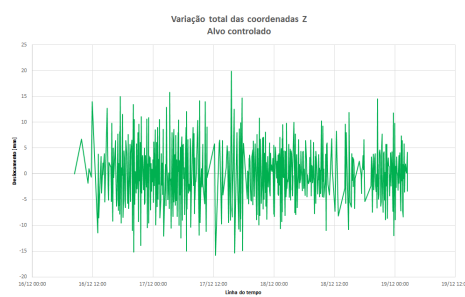

(c) Coordenada $Z_{P}$.

Figura 9.27: Posições das coordenadas $X_{P}, Y_{P}$ e $Z_{P}$ do braço robótico em todo o experimento.

Na sequência, a Figura 9.28 mostra as movimentações que foram detectadas pelo método iterativo de aproximações sucessivas com o uso de duas estações totais robóticas, durante todo o experimento de monitoramento. Para melhor visualização, a Figura 9.28a mostra a movimentação aplicada para a coordenada $X_{P}$, a Figura 9.28 b para a coordenada $Y_{P}$ e a Figura $9.28 \mathrm{c}$ para a coordenada $Z_{P}$.

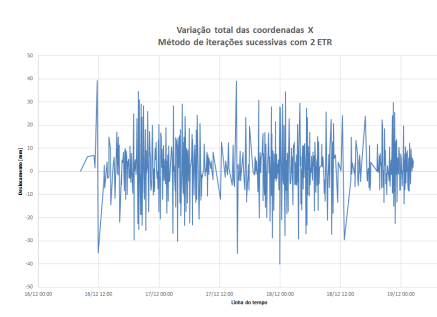

(a) Coordenada $X_{P}$.

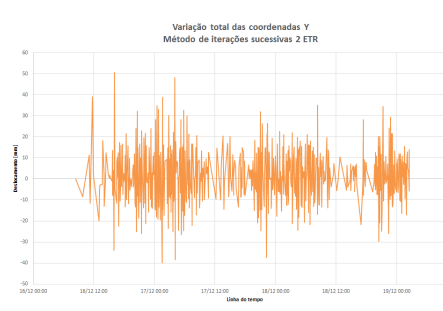

(b) Coordenada $Y_{P}$.

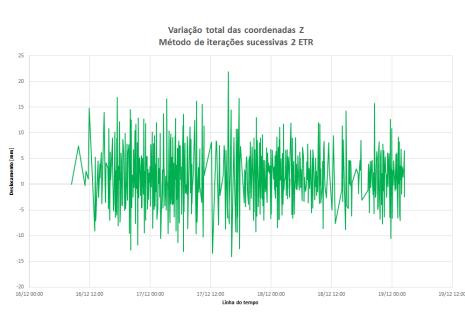

(c) Coordenada $Z_{P}$.

Figura 9.28: Posições das coordenadas $X_{P}, Y_{P}$ e $Z_{P}$ do método iterativo com 2 ETR em todo o experimento.

A Figura 9.29 mostra as movimentações que foram detectadas pelo método iterativo de aproximações sucessivas com o uso de três estações totais robóticas, durante todo o experimento de monitoramento. E, para melhor visualização, a Figura 9.29a mostra a movimentação aplicada para a coordenada $X_{P}$, a Figura 9.29b para a coordenada $Y_{P}$ e a Figura 9.29c para a coordenada $Z_{P}$. 


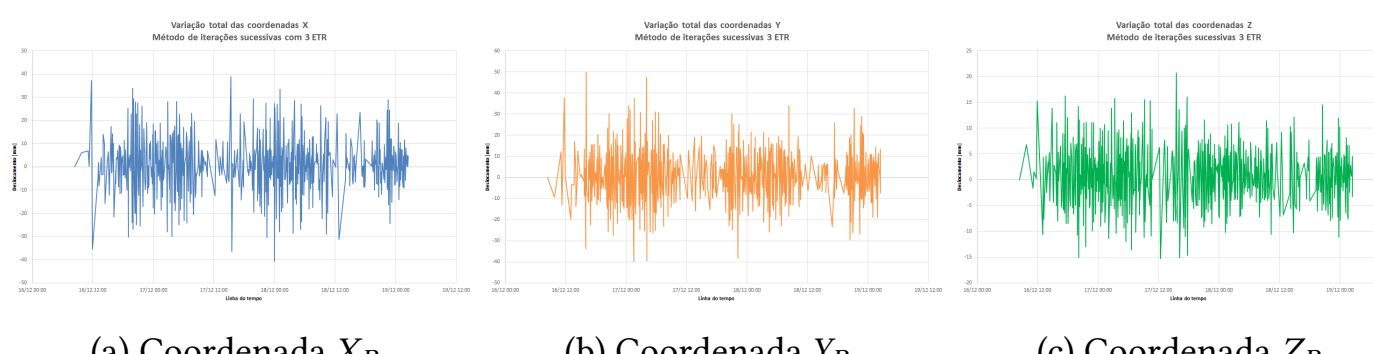
(a) Coordenada $X_{P}$.
(b) Coordenada $Y_{P}$.
(c) Coordenada $Z_{P}$.

Figura 9.29: Posições das coordenadas $X_{P}, Y_{P}$ e $Z_{P}$ do método iterativo com 3 ETR em todo o experimento.

Outro resultado importante para a validação dos métodos iterativos de aproximações sucessivas com o uso de duas e de três estações totais robóticas é a convergência. Ressaltando-se que é característica do modelo matemático de Newton Generalizado, comforme Demidovich (1981), que as coordenadas calculadas dentro de um loop iterativo, devem convergir para o valor mais aproximado possível de um valor verdadeiro, ou considerado como.

Dessa forma, foram extraídos do sistema de monitoramento proposto, especificamente, do subsistema de processamento, alguns gráficos que mostram a convergência obtida por cada método proposto, separadamente em cada eixo das coordenadas espaciais calculadas.

Dessa forma, a Figura 9.30 mostra a visualização gráfica da convergência obtida pelo método iterativo de aproximações sucessivas com o uso de duas estações totais robóticas.

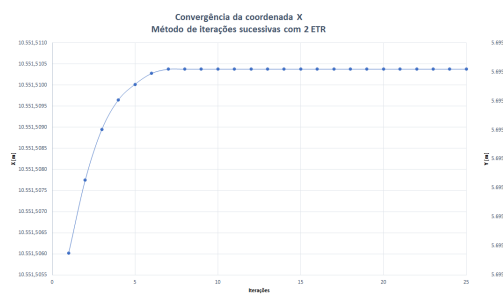

(a) Convergência de $X_{P}$.

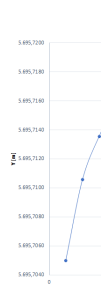

(b) Convergência de $Y_{P}$.
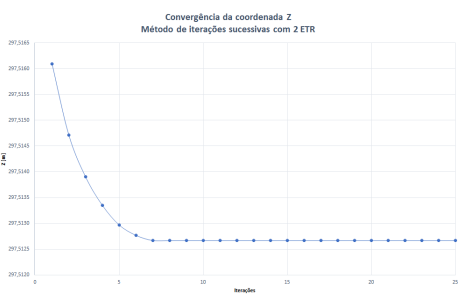

(c) Convergência de $Z_{P}$.

Figura 9.30: Convergência das coordenadas $X_{P}, Y_{P}$ e $Z_{P}$ do método iterativo com 2 ETR.

E por fim, a Figura 9.31 mostra a visualização gráfica da convergência obtida pelo método iterativo de aproximações sucessivas com o uso de três estações totais robóticas.

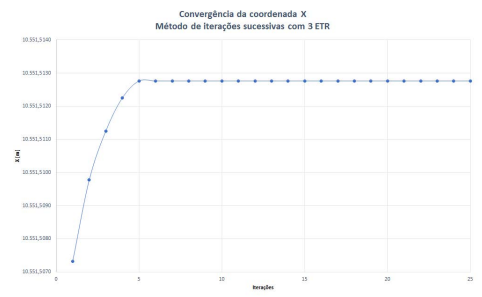

(a) Convergência de $X_{P}$.

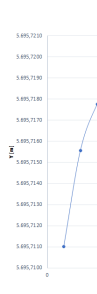

(b) Convergência de $Y_{P}$.

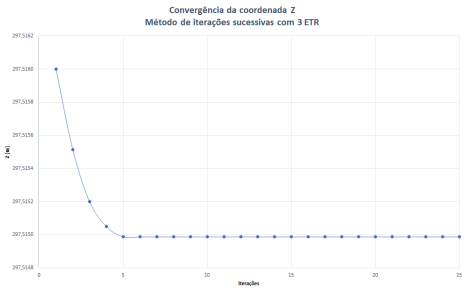

(c) Convergência de $Z_{P}$.

Figura 9.31: Convergência das coordenadas $X_{P}, Y_{P}$ e $Z_{P}$ do método iterativo com 3 ETR. 


\subsection{Análises comparativas}

Para efeito comparativo entre todos os métodos que foram validados, com o objetivo de garantir sua aplicação em sistemas de monitoramento geodésico de estruturas, foram considerados os resultados obtidos na seção 9.2 .

Com a finalidade de realizar as análises comparativas, foi considerada, como referencial ou valor absoluto, a movimentação em ciclos contínuos de cinco minutos, executadas pelo robô.

Assim, as análises entre os resultados obtidos foram efetuadas com a comparação entre as coordenadas do alvo controlado pelo robô e as coordenadas espaciais obtidas por cada método, bem como suas respectivas precisões. De posse destes dados, foi possível calcular a variação das coordenadas e verificar a dimensão dos deslocamentos que cada método, aqui considerado, pode detectar.

Na sequência, são apresentados os resíduos que foram obtidos por meio das diferenças absolutas entre a variação do alvo controlado e as variações entre as coordenadas espaciais determinadas por cada método. Trata-se, portanto, da acurácia que cada método de determinação de coordenadas espaciais possui.

Os cálculos foram realizados com todas as casas decimais possíveis no ambiente Python e, por este motivo, eles podem divergir de outras ferramentas computacionais. Ressalta-se que, ainda foram realizados arredondamentos, para se obter conformidade com os níveis de significância das medições e ajustamentos.

A Tabela 9.20 mostra o comportamento do método polar com média, perante a movimentação executada pelo robô, considerando-se as amostras agrupadas.

Tabela 9.20: Deslocamentos e acurácias obtidas pela média do método polar.

\begin{tabular}{|c|c|c|c|c|c|c|c|c|c|}
\hline \multirow{2}{*}{$\begin{array}{c}\text { Tempo } \\
{[\text { Data } / \text { Hora }]}\end{array}$} & \multicolumn{3}{|c|}{ Variação do controle } & \multicolumn{3}{|c|}{ Variação das coordenadas } & \multicolumn{3}{|c|}{ Acurácia } \\
\hline & $X[\mathrm{~mm}]$ & $Y[\mathrm{~mm}]$ & $Z[\mathrm{~mm}]$ & $X[\mathrm{~mm}]$ & $Y[\mathrm{~mm}]$ & $Z[\mathrm{~mm}]$ & $X[m m]$ & $Y[\mathrm{~mm}]$ & $Z[\mathbf{m m}]$ \\
\hline 16/12/2018 14:51:09 & 0,83 & 0,15 & 13,97 & 1,39 & $-0,02$ & 12,68 & 0,56 & 0,17 & 1,29 \\
\hline 16/12/2018 14:56:12 & 5,88 & $-1,50$ & 4,19 & 5,20 & $-0,94$ & $-2,20$ & 0,69 & 0,56 & 6,39 \\
\hline 16/12/2018 15:01:11 & $-2,03$ & 4,60 & $-8,70$ & $-1,54$ & 0,49 & $-5,11$ & 0,49 & 4,11 & 3,58 \\
\hline 16/12/2018 15:06:12 & $-9,84$ & $-3,66$ & 1,76 & $-12,83$ & $-1,16$ & 2,14 & 3,00 & 2,49 & 0,37 \\
\hline 16/12/2018 15:11:11 & 32,90 & 2,24 & $-3,11$ & 37,19 & 2,87 & $-3,65$ & 4,29 & 0,62 & 0,54 \\
\hline 17/12/2018 01:01:07 & 1,31 & 1,59 & $-2,74$ & $-2,44$ & 0,52 & 2,85 & 3,75 & 1,07 & 5,59 \\
\hline 17/12/2018 01:06:06 & $-0,91$ & $-21,37$ & 6,25 & 2,70 & $-20,84$ & $-1,96$ & 3,61 & 0,53 & 8,21 \\
\hline 17/12/2018 01:11:07 & $-1,67$ & 10,64 & 0,37 & $-6,01$ & 10,90 & 10,51 & 4,34 & 0,26 & 10,14 \\
\hline 17/12/2018 01:16:08 & 13,91 & 1,37 & $-3,55$ & 17,77 & 2,31 & $-8,51$ & 3,86 & 0,94 & 4,95 \\
\hline 17/12/2018 01:21:07 & $-11,69$ & $-17,99$ & $-5,15$ & $-13,62$ & $-18,98$ & $-5,14$ & 1,94 & 0,99 & 0,01 \\
\hline 17/12/2018 06:46:10 & $-2,93$ & 18,39 & 0,60 & $-3,46$ & 19,89 & $-0,14$ & 0,53 & 1,50 & 0,74 \\
\hline 17/12/2018 06:51:08 & $-13,05$ & $-13,84$ & 5,25 & $-11,13$ & $-15,04$ & 6,67 & 1,92 & 1,20 & 1,41 \\
\hline 17/12/2018 06:56:10 & 7,12 & 6,72 & $-7,00$ & 5,54 & 6,74 & $-3,71$ & 1,59 & 0,02 & 3,29 \\
\hline 17/12/2018 07:01:14 & 10,28 & $-4,07$ & 6,40 & 9,97 & $-3,25$ & $-1,96$ & 0,30 & 0,82 & 8,35 \\
\hline 17/12/2018 07:06:10 & $-8,00$ & $-10,58$ & 2,20 & $-7,33$ & $-13,08$ & 4,01 & 0,67 & 2,49 & 1,81 \\
\hline 18/12/2018 21:01:06 & $-5,10$ & $-18,65$ & 2,40 & $-3,17$ & $-15,91$ & 3,42 & 1,93 & 2,74 & 1,02 \\
\hline 18/12/2018 21:06:08 & $-9,61$ & 10,41 & $-10,35$ & $-11,90$ & 7,87 & $-6,30$ & 2,28 & 2,54 & 4,05 \\
\hline 18/12/2018 21:11:06 & $-0,45$ & 29,27 & $-5,20$ & 0,92 & 32,31 & $-5,43$ & 1,37 & 3,04 & 0,24 \\
\hline 18/12/2018 21:16:07 & 0,77 & $-10,28$ & 7,68 & 0,96 & $-12,19$ & 5,53 & 0,19 & 1,91 & 2,15 \\
\hline
\end{tabular}


A técnica de determinação de coordenadas do método abaixo é similar ao polar, porém com aplicação de ajustamento. Dessa forma, a Tabela 9.21 mostra o comportamento do método polar com ajuste das observações perante a movimentação executada pelo robô, considerandose as amostras agrupadas.

Tabela 9.21: Deslocamentos e acurácias obtidas pelo método polar com ajuste das observações.

\begin{tabular}{|c|c|c|c|c|c|c|c|c|c|}
\hline \multirow{2}{*}{$\frac{\text { Tempo }}{[\text { Data/Hora] }}$} & \multicolumn{3}{|c|}{ Variação do controle } & \multicolumn{3}{|c|}{ Variação das coordenadas } & \multicolumn{3}{|c|}{ Acurácia } \\
\hline & $X[\mathrm{~mm}]$ & $Y[\mathrm{~mm}]$ & $Z[\mathrm{~mm}]$ & $X[\mathrm{~mm}]$ & $Y[\mathrm{~mm}]$ & $Z[\mathrm{~mm}]$ & $X[\mathrm{~mm}]$ & $Y[\mathrm{~mm}]$ & $Z[\mathbf{m m}]$ \\
\hline 16/12/2018 14:26:09 & 0,83 & 0,15 & 13,97 & 1,39 & $-0,02$ & 12,68 & 0,56 & 0,17 & 1,29 \\
\hline 16/12/2018 14:56:12 & 5,88 & $-1,50$ & 4,19 & 5,20 & $-0,95$ & $-2,20$ & 0,68 & 0,55 & 6,39 \\
\hline 16/12/2018 15:01:11 & $-2,03$ & 4,60 & $-8,70$ & $-1,55$ & 0,50 & $-5,11$ & 0,48 & 4,11 & 3,58 \\
\hline 16/12/2018 15:06:12 & $-9,84$ & $-3,66$ & 1,76 & $-12,84$ & $-1,16$ & 2,14 & 3,00 & 2,50 & 0,37 \\
\hline 16/12/2018 15:11:11 & 32,90 & 2,24 & $-3,11$ & 37,20 & 2,86 & $-3,65$ & 4,30 & 0,62 & 0,54 \\
\hline 17/12/2018 01:01:07 & 1,31 & 1,59 & $-2,74$ & $-2,44$ & 0,52 & 2,85 & 3,75 & 1,07 & 5,59 \\
\hline 17/12/2018 01:06:06 & $-0,91$ & $-21,37$ & 6,25 & 2,70 & $-20,83$ & $-1,96$ & 3,61 & 0,54 & 8,21 \\
\hline 17/12/2018 01:11:07 & $-1,67$ & 10,64 & 0,37 & $-6,01$ & 10,90 & 10,51 & 4,34 & 0,26 & 10,14 \\
\hline 17/12/2018 01:16:08 & 13,91 & 1,37 & $-3,55$ & 17,77 & 2,31 & $-8,51$ & 3,86 & 0,94 & 4,95 \\
\hline 17/12/2018 01:21:07 & $-11,69$ & $-17,99$ & $-5,15$ & $-13,63$ & $-18,97$ & $-5,14$ & 1,94 & 0,99 & 0,01 \\
\hline 17/12/2018 06:46:10 & $-2,93$ & 18,39 & 0,60 & $-3,46$ & 19,89 & $-0,14$ & 0,53 & 1,50 & 0,74 \\
\hline 17/12/2018 06:51:08 & $-13,05$ & $-13,84$ & 5,25 & $-11,13$ & $-15,04$ & 6,67 & 1,92 & 1,20 & 1,41 \\
\hline 17/12/2018 06:56:10 & 7,12 & 6,72 & $-7,00$ & 5,54 & 6,74 & $-3,71$ & 1,59 & 0,02 & 3,29 \\
\hline 17/12/2018 07:01:14 & 10,28 & $-4,07$ & 6,40 & 9,97 & $-3,25$ & $-1,96$ & 0,30 & 0,82 & 8,35 \\
\hline 17/12/2018 07:06:10 & $-8,00$ & $-10,58$ & 2,20 & $-7,33$ & $-13,08$ & 4,01 & 0,67 & 2,49 & 1,81 \\
\hline 18/12/2018 21:01:06 & $-5,10$ & $-18,65$ & 2,40 & $-3,17$ & $-15,90$ & 3,42 & 1,93 & 2,74 & 1,02 \\
\hline 18/12/2018 21:06:08 & $-9,61$ & 10,41 & $-10,35$ & $-11,90$ & 7,88 & $-6,30$ & 2,28 & 2,53 & 4,05 \\
\hline 18/12/2018 21:11:06 & $-0,45$ & 29,27 & $-5,20$ & 0,92 & 32,29 & $-5,43$ & 1,37 & 3,02 & 0,24 \\
\hline 18/12/2018 21:16:07 & 0,77 & $-10,28$ & 7,68 & 0,96 & $-12,19$ & 5,53 & 0,19 & 1,91 & 2,15 \\
\hline
\end{tabular}

E, como se pode ver, os valores das coordenadas obtidas por ambos os métodos polares, com o valor médio e com ajustamento das observações, são muito próximos e se diferenciam apenas depois da sexta e da sétima casa decimal, o que os torna iguais, quando considerados os arredondamentos para os algarismos significativos. Dessa forma, o que os diferencia é obtenção das precisões das incógnitas medidas.

Na sequência, serão apresentados os métodos multipolares sem medição de distância. Notase que esses métodos não se mostram com aderência milimétrica em ralação ao controle e, consequentemente, são menos acurados. Porém, devido ao fato de se utilizarem de técnicas similares, para a obtenção das coordenadas espaciais, seguem, de certa forma, o mesmo padrão de acurácia, com aderências centimétricas. Isso, devido ao fato de não considerarem os efeitos atmosféricos. 
A Tabela 9.22 mostra o comportamento do método de interseção espacial trigonométrico perante a movimentação executada pelo robô, em todas as amostras consideradas.

Tabela 9.22: Deslocamentos e acurácias obtidas pelo método de interseção espacial trigonométrico.

\begin{tabular}{|c|c|c|c|c|c|c|c|c|c|}
\hline \multirow{2}{*}{$\frac{\text { Tempo }}{[\text { Data } / \text { Hora }]}$} & \multicolumn{3}{|c|}{ Variação do controle } & \multicolumn{3}{|c|}{ Variação das coordenadas } & \multicolumn{3}{|c|}{ Acurácia } \\
\hline & $X[\mathrm{~mm}]$ & $Y[\mathrm{~mm}]$ & $Z[\mathrm{~mm}]$ & $X[\mathrm{~mm}]$ & $Y[\mathrm{~mm}]$ & $Z[\mathrm{~mm}]$ & $X[\mathrm{~mm}]$ & $Y[\mathrm{~mm}]$ & $Z[\mathrm{~mm}]$ \\
\hline 16/12/2018 14:51:09 & 0,83 & 0,15 & 13,97 & 35,18 & 42,56 & 12,45 & 34,35 & 42,40 & 1,52 \\
\hline 16/12/2018 14:56:12 & 5,88 & $-1,50$ & 4,19 & $-48,16$ & $-57,93$ & $-1,66$ & 54,04 & 56,42 & 5,85 \\
\hline 16/12/2018 15:01:11 & $-2,03$ & 4,60 & $-8,70$ & 20,66 & 24,79 & $-5,33$ & 22,69 & 20,19 & 3,36 \\
\hline 16/12/2018 15:06:12 & $-9,84$ & $-3,66$ & 1,76 & 10,46 & 28,58 & 1,99 & 20,29 & 32,24 & 0,22 \\
\hline 16/12/2018 15:11:11 & 32,90 & 2,24 & $-3,11$ & $-210,11$ & $-362,40$ & $-2,46$ & 243,01 & 364,64 & 0,64 \\
\hline 17/12/2018 01:01:07 & 1,31 & 1,59 & $-2,74$ & 210,18 & 359,72 & $-3,21$ & 208,86 & 358,14 & 0,47 \\
\hline 17/12/2018 01:06:06 & $-0,91$ & $-21,37$ & 6,25 & 13,50 & $-14,45$ & 1,50 & 14,41 & 6,92 & 4,74 \\
\hline 17/12/2018 01:11:07 & $-1,67$ & 10,64 & 0,37 & $-219,28$ & $-353,67$ & 9,53 & 217,61 & 364,31 & 9,16 \\
\hline 17/12/2018 01:16:08 & 13,91 & 1,37 & $-3,55$ & 39,82 & 87,35 & $-10,18$ & 25,91 & 85,99 & 6,62 \\
\hline 17/12/2018 01:21:07 & $-11,69$ & $-17,99$ & $-5,15$ & $-46,81$ & $-94,24$ & $-0,07$ & 35,12 & 76,25 & 5,08 \\
\hline 17/12/2018 06:46:10 & $-2,93$ & 18,39 & 0,60 & 30,00 & 71,48 & $-0,21$ & 32,94 & 53,08 & 0,81 \\
\hline 17/12/2018 06:51:08 & $-13,05$ & $-13,84$ & 5,25 & $-27,61$ & $-48,64$ & 6,56 & 14,55 & 34,81 & 1,30 \\
\hline 17/12/2018 06:56:10 & 7,12 & 6,72 & $-7,00$ & $-25,23$ & $-26,33$ & $-3,38$ & 32,36 & 33,05 & 3,61 \\
\hline 17/12/2018 07:01:14 & 10,28 & $-4,07$ & 6,40 & 43,09 & 38,57 & $-2,20$ & 32,82 & 42,65 & 8,60 \\
\hline 17/12/2018 07:06:10 & $-8,00$ & $-10,58$ & 2,20 & 62,52 & 87,79 & 3,76 & 70,52 & 98,37 & 1,56 \\
\hline 18/12/2018 21:01:06 & $-5,10$ & $-18,65$ & 2,40 & $-72,97$ & $-120,72$ & 3,64 & 67,87 & 102,07 & 1,24 \\
\hline 18/12/2018 21:06:08 & $-9,61$ & 10,41 & $-10,35$ & $-100,63$ & $-116,16$ & $-5,87$ & 91,01 & 126,57 & 4,48 \\
\hline 18/12/2018 21:11:06 & $-0,45$ & 29,27 & $-5,20$ & 325,96 & 502,46 & $-6,71$ & 326,41 & 473,19 & 1,52 \\
\hline 18/12/2018 21:16:07 & 0,77 & $-10,28$ & 7,68 & $-7,42$ & $-22,24$ & 5,61 & 8,19 & 11,96 & 2,07 \\
\hline
\end{tabular}

A Tabela 9.23 mostra o comportamento do método do ponto médio da interseção espacial perante à movimentação executada pelo robô, em todas as amostras consideradas.

Tabela 9.23: Deslocamentos e acurácias obtidas pelo método do ponto médio da interseção espacial.

\begin{tabular}{|c|c|c|c|c|c|c|c|c|c|}
\hline \multirow{2}{*}{$\begin{array}{c}\text { Tempo } \\
{[\text { Data } / \text { Hora }]}\end{array}$} & \multicolumn{3}{|c|}{ Variação do controle } & \multicolumn{3}{|c|}{ Variação das coordenadas } & \multicolumn{3}{|c|}{ Acurácia } \\
\hline & $X[\mathrm{~mm}]$ & $Y[\mathrm{~mm}]$ & $Z[\mathrm{~mm}]$ & $X[\mathrm{~mm}]$ & $Y[\mathrm{~mm}]$ & $Z[\mathrm{~mm}]$ & $X[\mathrm{~mm}]$ & $Y[\mathrm{~mm}]$ & $Z[\mathrm{~mm}]$ \\
\hline 16/12/2018 14:51:09 & 0,83 & 0,15 & 13,97 & 34,62 & 42,06 & 12,53 & 33,79 & 41,90 & 1,44 \\
\hline 16/12/2018 14:56:12 & 5,88 & $-1,50$ & 4,19 & $-46,61$ & $-56,09$ & $-1,78$ & 52,49 & 54,59 & 5,97 \\
\hline 16/12/2018 15:01:11 & $-2,03$ & 4,60 & $-8,70$ & 19,92 & 24,12 & $-5,28$ & 21,95 & 19,51 & 3,42 \\
\hline 16/12/2018 15:06:12 & $-9,84$ & $-3,66$ & 1,76 & 10,28 & 28,34 & 2,04 & 20,12 & 32,00 & 0,28 \\
\hline 16/12/2018 15:11:11 & 32,90 & 2,24 & $-3,11$ & $-199,48$ & $-340,60$ & $-3,51$ & 232,38 & 342,84 & 0,41 \\
\hline 17/12/2018 01:01:07 & 1,31 & 1,59 & $-2,74$ & 18,93 & 37,52 & 2,96 & 17,62 & 35,94 & 5,70 \\
\hline 17/12/2018 01:06:06 & $-0,91$ & $-21,37$ & 6,25 & $-35,83$ & $-72,62$ & $-1,85$ & 34,92 & 51,25 & 8,10 \\
\hline 17/12/2018 01:11:07 & $-1,67$ & 10,64 & 0,37 & 23,68 & 50,69 & 10,42 & 25,35 & 40,05 & 10,04 \\
\hline 17/12/2018 01:16:08 & 13,91 & 1,37 & $-3,55$ & 2,24 & $-19,42$ & $-8,47$ & 11,66 & 20,79 & 4,92 \\
\hline 17/12/2018 01:21:07 & $-11,69$ & $-17,99$ & $-5,15$ & $-20,92$ & $-31,91$ & $-5,16$ & 9,23 & 13,92 & 0,01 \\
\hline 17/12/2018 06:46:10 & $-2,93$ & 18,39 & 0,60 & 30,39 & 71,87 & $-0,10$ & 33,32 & 53,47 & 0,70 \\
\hline 17/12/2018 06:51:08 & $-13,05$ & $-13,84$ & 5,25 & $-28,06$ & $-49,08$ & 6,47 & 15,00 & 35,25 & 1,21 \\
\hline 17/12/2018 06:56:10 & 7,12 & 6,72 & $-7,00$ & $-26,21$ & $-27,31$ & $-3,44$ & 33,33 & 34,03 & 3,55 \\
\hline 17/12/2018 07:01:14 & 10,28 & $-4,07$ & 6,40 & 42,89 & 38,40 & $-2,11$ & 32,62 & 42,47 & 8,51 \\
\hline 17/12/2018 07:06:10 & $-8,00$ & $-10,58$ & 2,20 & 65,09 & 90,39 & 3,93 & 73,10 & 100,98 & 1,73 \\
\hline 18/12/2018 21:01:06 & $-5,10$ & $-18,65$ & 2,40 & $-74,24$ & $-122,07$ & 3,41 & 69,14 & 103,42 & 1,01 \\
\hline 18/12/2018 21:06:08 & $-9,61$ & 10,41 & $-10,35$ & $-101,21$ & $-116,67$ & $-6,14$ & 91,59 & 127,08 & 4,21 \\
\hline 18/12/2018 21:11:06 & $-0,45$ & 29,27 & $-5,20$ & 327,12 & 503,50 & $-5,71$ & 327,57 & 474,24 & 0,51 \\
\hline 18/12/2018 21:16:07 & 0,77 & $-10,28$ & 7,68 & $-7,77$ & $-22,63$ & 5,60 & 8,54 & 12,34 & 2,09 \\
\hline
\end{tabular}


A Tabela 9.24 mostra o comportamento do método das distâncias mínimas perante à movimentação executada pelo robô, em todas as amostras consideradas.

Tabela 9.24: Deslocamentos e acurácias obtidas pelo método das distâncias mínimas.

\begin{tabular}{|c|c|c|c|c|c|c|c|c|c|}
\hline \multirow{2}{*}{$\frac{\text { Tempo }}{[\text { Data } / \text { Hora }]}$} & \multicolumn{3}{|c|}{ Variação do controle } & \multicolumn{3}{|c|}{ Variação das coordenadas } & \multicolumn{3}{|c|}{ Acurácia } \\
\hline & $X[\mathrm{~mm}]$ & $Y[\mathrm{~mm}]$ & $Z[\mathbf{m m}]$ & $X[\mathrm{~mm}]$ & $Y[m m]$ & $Z[\mathrm{~mm}]$ & $X[\mathrm{~mm}]$ & $Y[m m]$ & $Z[\mathrm{~mm}]$ \\
\hline 16/12/2018 14:51:09 & 0,83 & 0,15 & 13,97 & 9,83 & 8,46 & 12,59 & 8,99 & 8,30 & 1,38 \\
\hline 16/12/2018 14:56:12 & 5,88 & $-1,50$ & 4,19 & $-8,90$ & $-4,77$ & $-1,88$ & 14,78 & 3,26 & 6,07 \\
\hline 16/12/2018 15:01:11 & $-2,03$ & 4,60 & $-8,70$ & 1,81 & $-0,49$ & $-5,22$ & 3,84 & 5,09 & 3,47 \\
\hline 16/12/2018 15:06:12 & $-9,84$ & $-3,66$ & 1,76 & $-11,07$ & $-0,57$ & 2,10 & 1,24 & 3,08 & 0,34 \\
\hline 16/12/2018 15:11:11 & 32,90 & 2,24 & $-3,11$ & 64,65 & 8,84 & $-3,97$ & 31,75 & 6,60 & 0,86 \\
\hline 17/12/2018 01:01:07 & 1,31 & 1,59 & $-2,74$ & $-58,97$ & $-4,23$ & $-1,74$ & 60,28 & 5,81 & 0,99 \\
\hline 17/12/2018 01:06:06 & $-0,91$ & $-21,37$ & 6,25 & 14,61 & $-12,88$ & 1,50 & 15,52 & 8,49 & 4,75 \\
\hline 17/12/2018 01:11:07 & $-1,67$ & 10,64 & 0,37 & 43,92 & 2,08 & 8,10 & 45,58 & 8,56 & 7,72 \\
\hline 17/12/2018 01:16:08 & 13,91 & 1,37 & $-3,55$ & $-9,73$ & 20,33 & $-9,91$ & 23,63 & 18,97 & 6,36 \\
\hline 17/12/2018 01:21:07 & $-11,69$ & $-17,99$ & $-5,15$ & 5,65 & $-23,22$ & $-0,35$ & 17,34 & 5,24 & 4,80 \\
\hline 17/12/2018 06:46:10 & $-2,93$ & 18,39 & 0,60 & $-11,71$ & 15,05 & 0,02 & 8,78 & 3,34 & 0,58 \\
\hline 17/12/2018 06:51:08 & $-13,05$ & $-13,84$ & 5,25 & 6,55 & $-2,37$ & 6,37 & 19,61 & 11,46 & 1,12 \\
\hline 17/12/2018 06:56:10 & 7,12 & 6,72 & $-7,00$ & $-0,89$ & 6,73 & $-3,51$ & 8,02 & 0,01 & 3,48 \\
\hline 17/12/2018 07:01:14 & 10,28 & $-4,07$ & 6,40 & 9,59 & $-6,67$ & $-2,02$ & 0,69 & 2,60 & 8,42 \\
\hline 17/12/2018 07:06:10 & $-8,00$ & $-10,58$ & 2,20 & $-10,45$ & $-11,22$ & 4,15 & 2,44 & 0,63 & 1,95 \\
\hline 18/12/2018 21:01:06 & $-5,10$ & $-18,65$ & 2,40 & 16,35 & 0,12 & 3,16 & 21,45 & 18,77 & 0,75 \\
\hline 18/12/2018 21:06:08 & $-9,61$ & 10,41 & $-10,35$ & 1,97 & 22,74 & $-6,43$ & 11,59 & 12,33 & 3,92 \\
\hline 18/12/2018 21:11:06 & $-0,45$ & 29,27 & $-5,20$ & $-57,25$ & $-15,93$ & $-4,62$ & 56,80 & 45,20 & 0,57 \\
\hline 18/12/2018 21:16:07 & 0,77 & $-10,28$ & 7,68 & $-0,88$ & $-13,39$ & 5,58 & 1,65 & 3,10 & 2,11 \\
\hline
\end{tabular}

A Tabela 9.25 mostra o comportamento do método das distâncias mínimas com ajuste das observações perante à movimentação executada pelo robô, em todas as amostras consideradas.

Tabela 9.25: Deslocamentos e acurácias obtidas pelo método das distâncias mínimas com ajuste das observações.

\begin{tabular}{|c|c|c|c|c|c|c|c|c|c|}
\hline \multirow{2}{*}{$\begin{array}{c}\text { Tempo } \\
{[\text { Data/Hora] }}\end{array}$} & \multicolumn{3}{|c|}{ Variação do controle } & \multicolumn{3}{|c|}{ Variação das coordenadas } & \multicolumn{3}{|c|}{ Acurácia } \\
\hline & $X[\mathrm{~mm}]$ & $Y[\mathrm{~mm}]$ & $Z[\mathbf{m m}]$ & $X[\mathrm{~mm}]$ & $Y[\mathrm{~mm}]$ & $Z[\mathbf{m m}]$ & $X[\mathrm{~mm}]$ & $Y[m m]$ & $Z[\mathbf{m m}]$ \\
\hline 16/12/2018 14:51:09 & 0,83 & 0,15 & 13,97 & 9,83 & 8,46 & 12,59 & 8,99 & 8,30 & 1,38 \\
\hline 16/12/2018 14:56:12 & 5,88 & $-1,50$ & 4,19 & $-8,90$ & $-4,77$ & $-1,88$ & 14,78 & 3,26 & 6,07 \\
\hline 16/12/2018 15:01:11 & $-2,03$ & 4,60 & $-8,70$ & 1,81 & $-0,49$ & $-5,22$ & 3,84 & 5,09 & 3,47 \\
\hline 16/12/2018 15:06:12 & $-9,84$ & $-3,66$ & 1,76 & $-11,07$ & $-0,57$ & 2,10 & 1,24 & 3,08 & 0,34 \\
\hline 16/12/2018 15:11:11 & 32,90 & 2,24 & $-3,11$ & 64,65 & 8,84 & $-3,97$ & 31,75 & 6,60 & 0,86 \\
\hline 17/12/2018 01:01:07 & 1,31 & 1,59 & $-2,74$ & $-58,97$ & $-4,23$ & $-1,74$ & 60,28 & 5,81 & 0,99 \\
\hline 17/12/2018 01:06:06 & $-0,91$ & $-21,37$ & 6,25 & 14,61 & $-12,88$ & 1,50 & 15,52 & 8,49 & 4,75 \\
\hline 17/12/2018 01:11:07 & $-1,67$ & 10,64 & 0,37 & 43,92 & 2,08 & 8,10 & 45,58 & 8,56 & 7,72 \\
\hline 17/12/2018 01:16:08 & 13,91 & 1,37 & $-3,55$ & $-9,73$ & 20,33 & $-9,91$ & 23,63 & 18,97 & 6,36 \\
\hline 17/12/2018 01:21:07 & $-11,69$ & $-17,99$ & $-5,15$ & 5,65 & $-23,22$ & $-0,35$ & 17,34 & 5,24 & 4,80 \\
\hline 17/12/2018 06:46:10 & $-2,93$ & 18,39 & 0,60 & $-11,71$ & 15,05 & 0,02 & 8,78 & 3,34 & 0,58 \\
\hline 17/12/2018 06:51:08 & $-13,05$ & $-13,84$ & 5,25 & 6,55 & $-2,37$ & 6,37 & 19,61 & 11,46 & 1,12 \\
\hline 17/12/2018 06:56:10 & 7,12 & 6,72 & $-7,00$ & $-0,89$ & 6,73 & $-3,51$ & 8,02 & 0,01 & 3,48 \\
\hline 17/12/2018 07:01:14 & 10,28 & $-4,07$ & 6,40 & 9,59 & $-6,67$ & $-2,02$ & 0,69 & 2,60 & 8,42 \\
\hline 17/12/2018 07:06:10 & $-8,00$ & $-10,58$ & 2,20 & $-10,45$ & $-11,22$ & 4,15 & 2,44 & 0,63 & 1,95 \\
\hline 18/12/2018 21:01:06 & $-5,10$ & $-18,65$ & 2,40 & 16,35 & 0,12 & 3,16 & 21,45 & 18,77 & 0,75 \\
\hline 18/12/2018 21:06:08 & $-9,61$ & 10,41 & $-10,35$ & 1,97 & 22,74 & $-6,43$ & 11,59 & 12,33 & 3,92 \\
\hline 18/12/2018 21:11:06 & $-0,45$ & 29,27 & $-5,20$ & $-57,25$ & $-15,93$ & $-4,62$ & 56,80 & 45,20 & 0,57 \\
\hline 18/12/2018 21:16:07 & 0,77 & $-10,28$ & 7,68 & $-0,88$ & $-13,39$ & 5,58 & 1,65 & 3,10 & 2,11 \\
\hline
\end{tabular}


A Tabela 9.26 mostra o comportamento do método de interseção espacial analítica perante à movimentação executada pelo robô, em todas as amostras consideradas.

Tabela 9.26: Deslocamentos e acurácias obtidas pelo método de interseção espacial analítica.

\begin{tabular}{|c|c|c|c|c|c|c|c|c|c|}
\hline \multirow{2}{*}{$\frac{\text { Tempo }}{[\text { Data } / \text { Hora }]}$} & \multicolumn{3}{|c|}{ Variação do controle } & \multicolumn{3}{|c|}{ Variação das coordenadas } & \multicolumn{3}{|c|}{ Acurácia } \\
\hline & $X[\mathrm{~mm}]$ & $Y[\mathrm{~mm}]$ & $Z[\mathrm{~mm}]$ & $X[\mathrm{~mm}]$ & $Y[\mathrm{~mm}]$ & $Z[\mathrm{~mm}]$ & $X[m m]$ & $Y[\mathrm{~mm}]$ & $Z[\mathbf{m m}]$ \\
\hline 16/12/2018 14:51:09 & 0,83 & 0,15 & 13,97 & 34,65 & 42,02 & 12,46 & 33,82 & 41,87 & 1,51 \\
\hline 16/12/2018 14:56:12 & 5,88 & $-1,50$ & 4,19 & $-46,76$ & $-55,98$ & $-1,67$ & 52,65 & 54,48 & 5,86 \\
\hline 16/12/2018 15:01:11 & $-2,03$ & 4,60 & $-8,70$ & 19,97 & 24,07 & $-5,33$ & 22,00 & 19,47 & 3,37 \\
\hline 16/12/2018 15:06:12 & $-9,84$ & $-3,66$ & 1,76 & 10,30 & 28,33 & 1,99 & 20,14 & 31,98 & 0,22 \\
\hline 16/12/2018 15:11:11 & 32,90 & 2,24 & $-3,11$ & $-208,16$ & $-360,03$ & $-2,48$ & 241,06 & 362,27 & 0,63 \\
\hline 17/12/2018 01:01:07 & 1,31 & 1,59 & $-2,74$ & 209,50 & 358,76 & $-3,21$ & 208,19 & 357,17 & 0,47 \\
\hline 17/12/2018 01:06:06 & $-0,91$ & $-21,37$ & 6,25 & 13,76 & $-14,05$ & 1,50 & 14,67 & 7,32 & 4,75 \\
\hline 17/12/2018 01:11:07 & $-1,67$ & 10,64 & 0,37 & $-218,53$ & $-352,74$ & 9,52 & 216,86 & 363,38 & 9,15 \\
\hline 17/12/2018 01:16:08 & 13,91 & 1,37 & $-3,55$ & 39,67 & 87,12 & $-10,18$ & 25,76 & 85,75 & 6,62 \\
\hline 17/12/2018 01:21:07 & $-11,69$ & $-17,99$ & $-5,15$ & $-47,69$ & $-95,31$ & $-0,06$ & 36,01 & 77,33 & 5,09 \\
\hline 17/12/2018 06:46:10 & $-2,93$ & 18,39 & 0,60 & 30,35 & 71,89 & $-0,21$ & 33,28 & 53,50 & 0,81 \\
\hline 17/12/2018 06:51:08 & $-13,05$ & $-13,84$ & 5,25 & $-28,02$ & $-49,11$ & 6,56 & 14,97 & 35,28 & 1,31 \\
\hline 17/12/2018 06:56:10 & 7,12 & 6,72 & $-7,00$ & $-26,13$ & $-27,38$ & $-3,38$ & 33,25 & 34,09 & 3,62 \\
\hline 17/12/2018 07:01:14 & 10,28 & $-4,07$ & 6,40 & 42,90 & 38,39 & $-2,20$ & 32,63 & 42,46 & 8,60 \\
\hline 17/12/2018 07:06:10 & $-8,00$ & $-10,58$ & 2,20 & 64,88 & 90,57 & 3,74 & 72,88 & 101,15 & 1,54 \\
\hline 18/12/2018 21:01:06 & $-5,10$ & $-18,65$ & 2,40 & $-74,12$ & $-122,16$ & 3,65 & 69,02 & 103,52 & 1,25 \\
\hline 18/12/2018 21:06:08 & $-9,61$ & 10,41 & $-10,35$ & $-101,16$ & $-116,71$ & $-5,86$ & 91,55 & 127,12 & 4,48 \\
\hline 18/12/2018 21:11:06 & $-0,45$ & 29,27 & $-5,20$ & 327,01 & 503,59 & $-6,72$ & 327,46 & 474,32 & 1,53 \\
\hline 18/12/2018 21:16:07 & 0,77 & $-10,28$ & 7,68 & $-7,74$ & $-22,65$ & 5,61 & 8,51 & 12,37 & 2,07 \\
\hline
\end{tabular}


Na sequência, apresentam-se as análises comparativas, considerando os métodos multipolares com medições de distâncias.

Primeiramente, consideram-se os métodos multipolares com medições de distâncias, especificamente os de minimização dos ângulos e das distâncias medidas, que também se embasam no método polar para determinação de coordenadas espaciais. Dessa forma, apresentam-se iguais aos métodos polares com média e com ajustamento, e diferenciam-se, igualmente, pela maneira com a qual realizam os ajustamentos das observações. Assim, consequentemente, os resultados obtidos das coordenadas espaciais, o grau de aderência ao controle e a acurácia também podem ser considerados iguais.

A Tabela 9.27 mostra o comportamento do método da minimização dos deslocamentos em função dos azimutes, dos ângulos verticais e das distâncias inclinadas medidas (relação geométrica) perante a movimentação executada pelo robô, em todas as amostras consideradas.

Tabela 9.27: Deslocamentos e acurácias obtidas pelo método da minimização dos deslocamentos em função dos azimutes, dos ângulos verticais e das distâncias inclinadas medidas (relação geométrica).

\begin{tabular}{|c|c|c|c|c|c|c|c|c|c|}
\hline \multirow{2}{*}{$\begin{array}{c}\text { Tempo } \\
{[\text { Data } / \text { Hora }]}\end{array}$} & \multicolumn{3}{|c|}{ Variação do controle } & \multicolumn{3}{|c|}{ Variação das coordenadas } & \multicolumn{3}{|c|}{ Acurácia } \\
\hline & $X[\mathrm{~mm}]$ & $Y[m m]$ & $Z[\mathrm{~mm}]$ & $X[\mathrm{~mm}]$ & $Y[m m]$ & $Z[\mathrm{~mm}]$ & $X[\mathrm{~mm}]$ & $Y[m m]$ & $Z[\mathrm{~mm}]$ \\
\hline 16/12/2018 14:51:09 & 0,83 & 0,15 & 13,97 & 1,39 & $-0,02$ & 12,68 & 0,56 & 0,17 & 1,29 \\
\hline 16/12/2018 14:56:12 & 5,88 & $-1,50$ & 4,19 & 5,20 & $-0,94$ & $-2,20$ & 0,69 & 0,56 & 6,39 \\
\hline 16/12/2018 15:01:11 & $-2,03$ & 4,60 & $-8,70$ & $-1,54$ & 0,49 & $-5,11$ & 0,49 & 4,11 & 3,58 \\
\hline 16/12/2018 15:06:12 & $-9,84$ & $-3,66$ & 1,76 & $-12,83$ & $-1,16$ & 2,14 & 3,00 & 2,49 & 0,37 \\
\hline 16/12/2018 15:11:11 & 32,90 & 2,24 & $-3,11$ & 37,19 & 2,87 & $-3,65$ & 4,29 & 0,62 & 0,54 \\
\hline 17/12/2018 01:01:07 & 1,31 & 1,59 & $-2,74$ & $-2,44$ & 0,52 & 2,85 & 3,75 & 1,07 & 5,59 \\
\hline 17/12/2018 01:06:06 & $-0,91$ & $-21,37$ & 6,25 & 2,70 & $-20,84$ & $-1,96$ & 3,61 & 0,53 & 8,21 \\
\hline 17/12/2018 01:11:07 & $-1,67$ & 10,64 & 0,37 & $-6,01$ & 10,90 & 10,51 & 4,34 & 0,26 & 10,14 \\
\hline 17/12/2018 01:16:08 & 13,91 & 1,37 & $-3,55$ & 17,77 & 2,31 & $-8,51$ & 3,86 & 0,94 & 4,95 \\
\hline 17/12/2018 01:21:07 & $-11,69$ & $-17,99$ & $-5,15$ & $-13,62$ & $-18,98$ & $-5,14$ & 1,94 & 0,99 & 0,01 \\
\hline 17/12/2018 06:46:10 & $-2,93$ & 18,39 & 0,60 & $-3,46$ & 19,89 & $-0,14$ & 0,53 & 1,50 & 0,74 \\
\hline 17/12/2018 06:51:08 & $-13,05$ & $-13,84$ & 5,25 & $-11,13$ & $-15,04$ & 6,67 & 1,92 & 1,20 & 1,41 \\
\hline 17/12/2018 06:56:10 & 7,12 & 6,72 & $-7,00$ & 5,54 & 6,74 & $-3,71$ & 1,59 & 0,02 & 3,29 \\
\hline 17/12/2018 07:01:14 & 10,28 & $-4,07$ & 6,40 & 9,97 & $-3,25$ & $-1,96$ & 0,30 & 0,82 & 8,35 \\
\hline 17/12/2018 07:06:10 & $-8,00$ & $-10,58$ & 2,20 & $-7,33$ & $-13,08$ & 4,01 & 0,67 & 2,49 & 1,81 \\
\hline 18/12/2018 21:01:06 & $-5,10$ & $-18,65$ & 2,40 & $-3,17$ & $-15,91$ & 3,42 & 1,93 & 2,74 & 1,02 \\
\hline 18/12/2018 21:06:08 & $-9,61$ & 10,41 & $-10,35$ & $-11,90$ & 7,87 & $-6,30$ & 2,28 & 2,54 & 4,05 \\
\hline 18/12/2018 21:11:06 & $-0,45$ & 29,27 & $-5,20$ & 0,92 & 32,31 & $-5,43$ & 1,37 & 3,04 & 0,24 \\
\hline 18/12/2018 21:16:07 & 0,77 & $-10,28$ & 7,68 & 0,96 & $-12,19$ & 5,53 & 0,19 & 1,91 & 2,15 \\
\hline
\end{tabular}


A Tabela 9.28 mostra o comportamento do método da minimização dos resíduos das funções trigonométricas em função dos azimutes, dos ângulos verticais e distâncias inclinadas medidas (relação analítica) perante a movimentação executada pelo robô, em todas as amostras consideradas.

Tabela 9.28: Deslocamentos e acurácias obtidas pelo método da minimização dos resíduos das funções trigonométricas em função dos azimutes, dos ângulos verticais e distâncias inclinadas medidas (relação analítica).

\begin{tabular}{|c|c|c|c|c|c|c|c|c|c|}
\hline \multirow{2}{*}{$\begin{array}{c}\text { Tempo } \\
{[\text { Data/Hora }]}\end{array}$} & \multicolumn{3}{|c|}{ Variação do controle } & \multicolumn{3}{|c|}{ Variação das coordenadas } & \multicolumn{3}{|c|}{ Acurácia } \\
\hline & $X[\mathrm{~mm}]$ & $Y[\mathrm{~mm}]$ & $Z[\mathbf{m m}]$ & $X[\mathrm{~mm}]$ & $Y[\mathrm{~mm}]$ & $Z[\mathrm{~mm}]$ & $X[m m]$ & $Y[\mathrm{~mm}]$ & $Z[\mathrm{~mm}]$ \\
\hline 16/12/2018 14:51:09 & 0,83 & 0,15 & 13,97 & 1,39 & $-0,02$ & 12,68 & 0,56 & 0,17 & 1,29 \\
\hline 16/12/2018 14:56:12 & 5,88 & $-1,50$ & 4,19 & 5,20 & $-0,94$ & $-2,20$ & 0,69 & 0,56 & 6,39 \\
\hline 16/12/2018 15:01:11 & $-2,03$ & 4,60 & $-8,70$ & $-1,54$ & 0,49 & $-5,11$ & 0,49 & 4,11 & 3,58 \\
\hline 16/12/2018 15:06:12 & $-9,84$ & $-3,66$ & 1,76 & $-12,83$ & $-1,16$ & 2,14 & 3,00 & 2,49 & 0,37 \\
\hline 16/12/2018 15:11:11 & 32,90 & 2,24 & $-3,11$ & 37,19 & 2,87 & $-3,65$ & 4,29 & 0,62 & 0,54 \\
\hline 17/12/2018 01:01:07 & 1,31 & 1,59 & $-2,74$ & $-2,44$ & 0,52 & 2,85 & 3,75 & 1,07 & 5,59 \\
\hline 17/12/2018 01:06:06 & $-0,91$ & $-21,37$ & 6,25 & 2,70 & $-20,84$ & $-1,96$ & 3,61 & 0,53 & 8,21 \\
\hline 17/12/2018 01:11:07 & $-1,67$ & 10,64 & 0,37 & $-6,01$ & 10,90 & 10,51 & 4,34 & 0,26 & 10,14 \\
\hline 17/12/2018 01:16:08 & 13,91 & 1,37 & $-3,55$ & 17,77 & 2,31 & $-8,51$ & 3,86 & 0,94 & 4,95 \\
\hline 17/12/2018 01:21:07 & $-11,69$ & $-17,99$ & $-5,15$ & $-13,62$ & $-18,98$ & $-5,14$ & 1,94 & 0,99 & 0,01 \\
\hline 17/12/2018 06:46:10 & $-2,93$ & 18,39 & 0,60 & $-3,46$ & 19,89 & $-0,14$ & 0,53 & 1,50 & 0,74 \\
\hline 17/12/2018 06:51:08 & $-13,05$ & $-13,84$ & 5,25 & $-11,13$ & $-15,04$ & 6,67 & 1,92 & 1,20 & 1,41 \\
\hline 17/12/2018 06:56:10 & 7,12 & 6,72 & $-7,00$ & 5,54 & 6,74 & $-3,71$ & 1,59 & 0,02 & 3,29 \\
\hline 17/12/2018 07:01:14 & 10,28 & $-4,07$ & 6,40 & 9,97 & $-3,25$ & $-1,96$ & 0,30 & 0,82 & 8,35 \\
\hline 17/12/2018 07:06:10 & $-8,00$ & $-10,58$ & 2,20 & $-7,33$ & $-13,08$ & 4,01 & 0,67 & 2,49 & 1,81 \\
\hline 18/12/2018 21:01:06 & $-5,10$ & $-18,65$ & 2,40 & $-3,17$ & $-15,91$ & 3,42 & 1,93 & 2,74 & 1,02 \\
\hline 18/12/2018 21:06:08 & $-9,61$ & 10,41 & $-10,35$ & $-11,90$ & 7,87 & $-6,30$ & 2,28 & 2,54 & 4,05 \\
\hline 18/12/2018 21:11:06 & $-0,45$ & 29,27 & $-5,20$ & 0,92 & 32,31 & $-5,43$ & 1,37 & 3,04 & 0,24 \\
\hline 18/12/2018 21:16:07 & 0,77 & $-10,28$ & 7,68 & 0,96 & $-12,19$ & 5,53 & 0,19 & 1,91 & 2,15 \\
\hline
\end{tabular}


Dando continuidade à análise comparativa, consideram-se abaixo os métodos multipolares com medição de distância, com técnicas iterativas de convergência quadrática, os quais se mostram muito mais acurados e com maior grau de aderência ao controle.

A Tabela 9.29 mostra o comportamento do método iterativo de aproximações sucessivas para determinação de coordenadas espaciais considerando duas estações totais robóticas perante a movimentação executada pelo robô, em todas as amostras consideradas.

Tabela 9.29: Deslocamentos e acurácias obtidas pelo método iterativo de aproximações sucessivas, para determinação de coordenadas espaciais, considerando duas estações totais robóticas.

\begin{tabular}{|c|c|c|c|c|c|c|c|c|c|}
\hline \multirow{2}{*}{$\frac{\text { Tempo }}{[\text { Data } / \text { Hora }]}$} & \multicolumn{3}{|c|}{ Variação do controle } & \multicolumn{3}{|c|}{ Variação das coordenadas } & \multicolumn{3}{|c|}{ Acurácia } \\
\hline & $X[\mathrm{~mm}]$ & $Y[\mathrm{~mm}]$ & $Z[\mathrm{~mm}]$ & $X[\mathrm{~mm}]$ & $Y[\mathrm{~mm}]$ & $Z[\mathrm{~mm}]$ & $X[\mathrm{~mm}]$ & $Y[\mathrm{~mm}]$ & $Z[\mathrm{~mm}]$ \\
\hline 16/12/2018 14:51:09 & 0,83 & 0,15 & 13,97 & 0,68 & $-0,35$ & 13,55 & 0,15 & 0,50 & 0,41 \\
\hline 16/12/2018 14:56:12 & 5,88 & $-1,50$ & 4,19 & 6,76 & $-3,24$ & 3,50 & 0,88 & 1,74 & 0,69 \\
\hline 16/12/2018 15:01:11 & $-2,03$ & 4,60 & $-8,70$ & $-2,10$ & 5,60 & $-9,77$ & 0,07 & 1,00 & 1,07 \\
\hline 16/12/2018 15:06:12 & $-9,84$ & $-3,66$ & 1,76 & $-11,29$ & $-2,56$ & 2,73 & 1,45 & 1,09 & 0,97 \\
\hline 16/12/2018 15:11:11 & 32,90 & 2,24 & $-3,11$ & 34,50 & 0,53 & $-2,25$ & 1,60 & 1,72 & 0,86 \\
\hline 17/12/2018 01:01:07 & 1,31 & 1,59 & $-2,74$ & 0,11 & 2,52 & $-3,19$ & 1,20 & 0,93 & 0,45 \\
\hline 17/12/2018 01:06:06 & $-0,91$ & $-21,37$ & 6,25 & 0,69 & $-22,83$ & 4,50 & 1,60 & 1,46 & 1,75 \\
\hline 17/12/2018 01:11:07 & $-1,67$ & 10,64 & 0,37 & $-2,84$ & 9,16 & 1,22 & 1,17 & 1,48 & 0,85 \\
\hline 17/12/2018 01:16:08 & 13,91 & 1,37 & $-3,55$ & 14,76 & 3,53 & $-1,69$ & 0,85 & 2,17 & 1,87 \\
\hline 17/12/2018 01:21:07 & $-11,69$ & $-17,99$ & $-5,15$ & $-12,60$ & $-16,91$ & $-4,14$ & 0,91 & 1,08 & 1,02 \\
\hline 17/12/2018 06:46:10 & $-2,93$ & 18,39 & 0,60 & $-1,87$ & 16,88 & $-1,87$ & 1,06 & 1,51 & 2,47 \\
\hline 17/12/2018 06:51:08 & $-13,05$ & $-13,84$ & 5,25 & $-15,02$ & $-11,35$ & 6,12 & 1,97 & 2,48 & 0,86 \\
\hline 17/12/2018 06:56:10 & 7,12 & 6,72 & $-7,00$ & 8,18 & 4,73 & $-8,03$ & 1,05 & 1,99 & 1,03 \\
\hline 17/12/2018 07:01:14 & 10,28 & $-4,07$ & 6,40 & 11,57 & $-2,53$ & 5,59 & 1,30 & 1,55 & 0,81 \\
\hline 17/12/2018 07:06:10 & $-8,00$ & $-10,58$ & 2,20 & $-9,78$ & $-12,66$ & 3,43 & 1,78 & 2,07 & 1,23 \\
\hline 18/12/2018 21:01:06 & $-5,10$ & $-18,65$ & 2,40 & $-3,48$ & $-16,19$ & 1,23 & 1,62 & 2,46 & 1,17 \\
\hline 18/12/2018 21:06:08 & $-9,61$ & 10,41 & $-10,35$ & $-10,86$ & 8,18 & $-9,29$ & 1,25 & 2,23 & 1,06 \\
\hline 18/12/2018 21:11:06 & $-0,45$ & 29,27 & $-5,20$ & 0,66 & 30,12 & $-4,20$ & 1,11 & 0,86 & 1,00 \\
\hline 18/12/2018 21:16:07 & 0,77 & $-10,28$ & 7,68 & 2,46 & $-9,11$ & 5,76 & 1,69 & 1,17 & 1,93 \\
\hline
\end{tabular}


A Tabela 9.30 mostra o comportamento do método iterativo de aproximações sucessivas para determinação de coordenadas espaciais considerando três estações totais robóticas perante a movimentação executada pelo robô, em todas as amostras consideradas.

Tabela 9.30: Deslocamentos e acurácias obtidas pelo método iterativo de aproximações sucessivas, para determinação de coordenadas espaciais, considerando três estações totais robóticas.

\begin{tabular}{|c|c|c|c|c|c|c|c|c|c|}
\hline \multirow{2}{*}{$\frac{\text { Tempo }}{[\text { Data } / \text { Hora }]}$} & \multicolumn{3}{|c|}{ Variação do controle } & \multicolumn{3}{|c|}{ Variação das coordenadas } & \multicolumn{3}{|c|}{ Acurácia } \\
\hline & $X[m m]$ & $Y[\mathrm{~mm}]$ & $Z[\mathrm{~mm}]$ & $X[m m]$ & $Y[\mathrm{~mm}]$ & $Z[\mathrm{~mm}]$ & $X[\mathrm{~mm}]$ & $Y[\mathrm{~mm}]$ & $Z[\mathrm{~mm}]$ \\
\hline 16/12/2018 14:51:09 & 0,83 & 0,15 & 13,97 & 0,63 & $-0,67$ & 14,01 & 0,20 & 0,83 & 0,04 \\
\hline 16/12/2018 14:56:12 & 5,88 & $-1,50$ & 4,19 & 6,48 & $-1,71$ & 4,52 & 0,60 & 0,20 & 0,33 \\
\hline 16/12/2018 15:01:11 & $-2,03$ & 4,60 & $-8,70$ & $-2,09$ & 4,92 & $-9,67$ & 0,06 & 0,32 & 0,97 \\
\hline 16/12/2018 15:06:12 & $-9,84$ & $-3,66$ & 1,76 & $-10,25$ & $-3,41$ & 1,81 & 0,42 & 0,25 & 0,05 \\
\hline 16/12/2018 15:11:11 & 32,90 & 2,24 & $-3,11$ & 33,28 & 2,14 & $-2,42$ & 0,38 & 0,10 & 0,69 \\
\hline 17/12/2018 01:01:07 & 1,31 & 1,59 & $-2,74$ & 0,95 & 1,48 & $-2,72$ & 0,36 & 0,11 & 0,01 \\
\hline 17/12/2018 01:06:06 & $-0,91$ & $-21,37$ & 6,25 & $-0,54$ & $-22,01$ & 5,02 & 0,37 & 0,64 & 1,23 \\
\hline 17/12/2018 01:11:07 & $-1,67$ & 10,64 & 0,37 & $-1,95$ & 10,83 & 1,24 & 0,28 & 0,19 & 0,87 \\
\hline 17/12/2018 01:16:08 & 13,91 & 1,37 & $-3,55$ & 13,75 & 1,13 & $-2,21$ & 0,15 & 0,23 & 1,35 \\
\hline 17/12/2018 01:21:07 & $-11,69$ & $-17,99$ & $-5,15$ & $-10,63$ & $-17,89$ & $-6,11$ & 1,05 & 0,10 & 0,96 \\
\hline 17/12/2018 06:46:10 & $-2,93$ & 18,39 & 0,60 & $-3,04$ & 19,54 & 0,80 & 0,11 & 1,15 & 0,20 \\
\hline 17/12/2018 06:51:08 & $-13,05$ & $-13,84$ & 5,25 & $-13,10$ & $-13,75$ & 4,28 & 0,05 & 0,09 & 0,98 \\
\hline 17/12/2018 06:56:10 & 7,12 & 6,72 & $-7,00$ & 6,79 & 6,06 & $-6,44$ & 0,34 & 0,66 & 0,55 \\
\hline 17/12/2018 07:01:14 & 10,28 & $-4,07$ & 6,40 & 10,79 & $-3,69$ & 6,17 & 0,51 & 0,38 & 0,23 \\
\hline 17/12/2018 07:06:10 & $-8,00$ & $-10,58$ & 2,20 & $-8,87$ & $-11,27$ & 1,96 & 0,86 & 0,69 & 0,24 \\
\hline 18/12/2018 21:01:06 & $-5,10$ & $-18,65$ & 2,40 & $-4,25$ & $-17,70$ & 2,04 & 0,85 & 0,95 & 0,36 \\
\hline 18/12/2018 21:06:08 & $-9,61$ & 10,41 & $-10,35$ & $-9,98$ & 9,33 & $-10,22$ & 0,37 & 1,08 & 0,13 \\
\hline 18/12/2018 21:11:06 & $-0,45$ & 29,27 & $-5,20$ & $-0,55$ & 29,69 & $-4,98$ & 0,10 & 0,42 & 0,21 \\
\hline 18/12/2018 21:16:07 & 0,77 & $-10,28$ & 7,68 & 1,39 & $-9,67$ & 7,26 & 0,62 & 0,61 & 0,43 \\
\hline
\end{tabular}


Para finalizar as análises comparativas, realiza-se, por meio de gráficos, uma análise dinâmica dos resultados obtidos pelos métodos propostos em relação ao controle. Dessa forma, apresentam-se os gráficos que mostram as variações das coordenadas espaciais e dos deslocamentos obtidos pelos dois métodos iterativos de aproximações, em comparação com o referencial absoluto, a movimentação exercida pelo robô.

A Figura 9.32 mostra a visualização gráfica da análise comparativa da variação coordenada $X$ entre o braço robótico e os dois métodos iterativos, com o uso de duas e três estações totais robóticas.

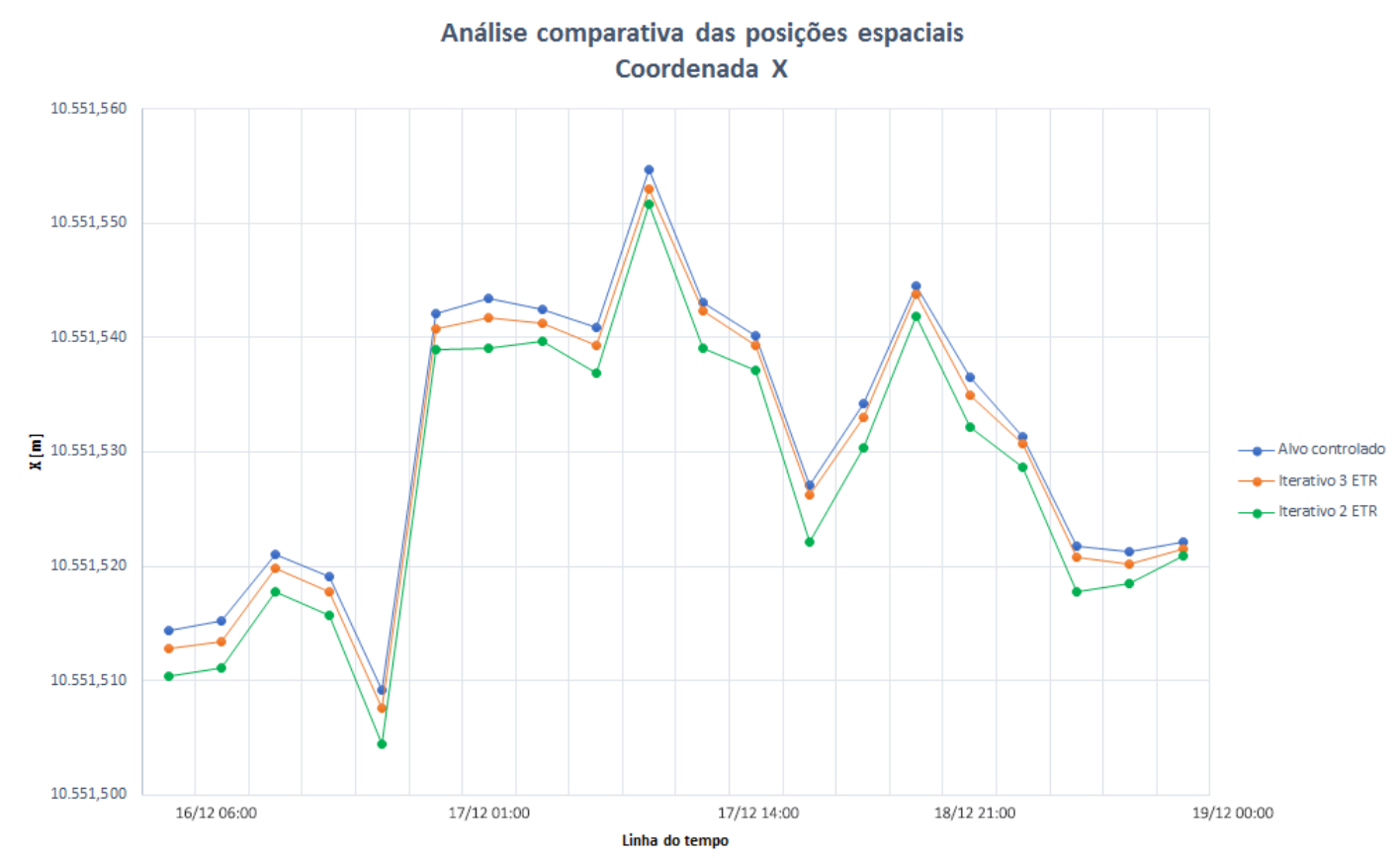

Figura 9.32: Análise comparativa da posição espacial $X_{P}$. 
A Figura 9.33 mostra a visualização gráfica da análise comparativa da variação coordenada $Y$ entre o braço robótico e os dois métodos iterativos, com o uso de duas e três estações totais robóticas.

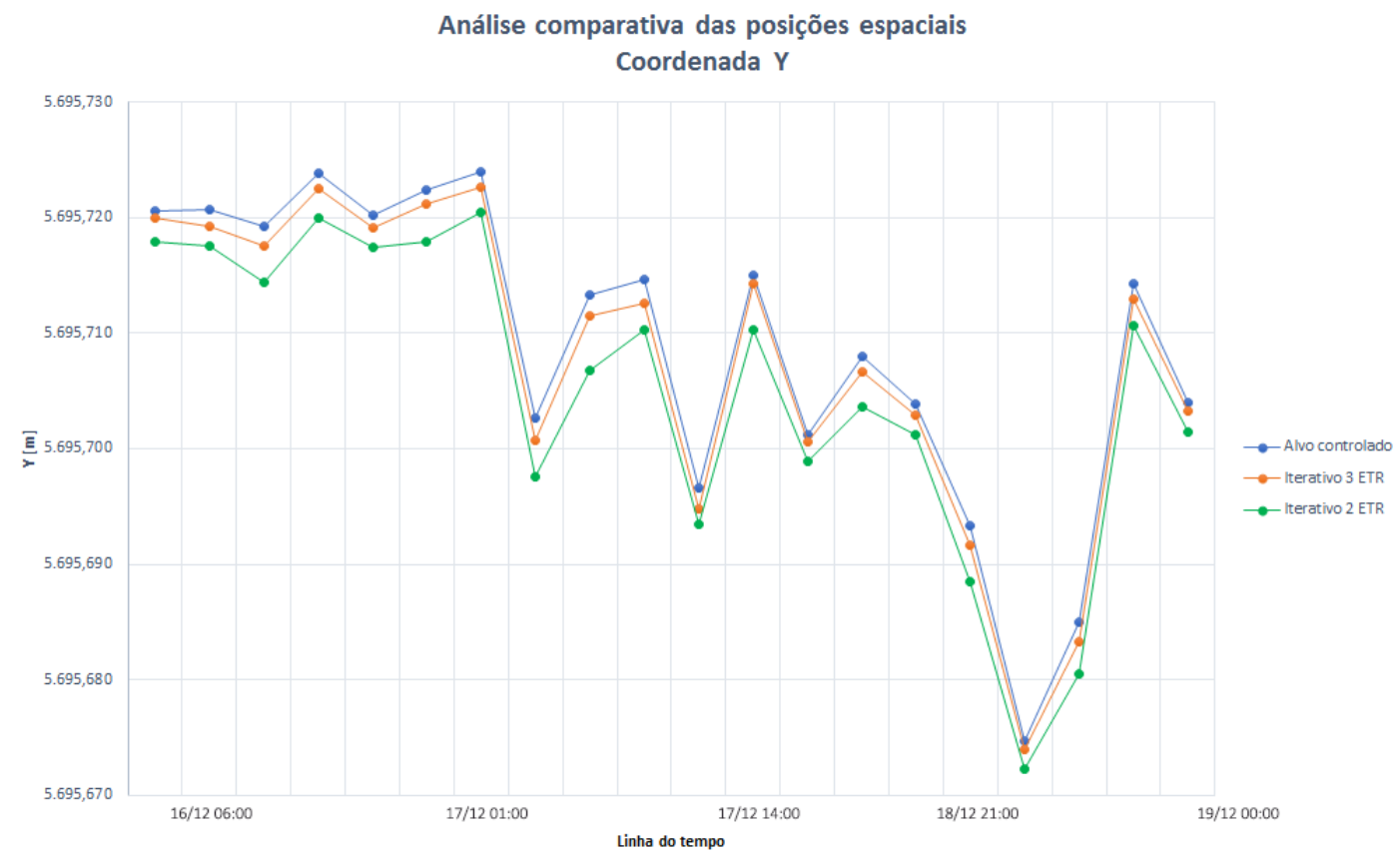

Figura 9.33: Análise comparativa da posição espacial $Y_{P}$.

A Figura 9.34 mostra a visualização gráfica da análise comparativa da variação coordenada $Z$ entre o braço robótico e os dois métodos iterativos, com o uso de duas e três estações totais robóticas.

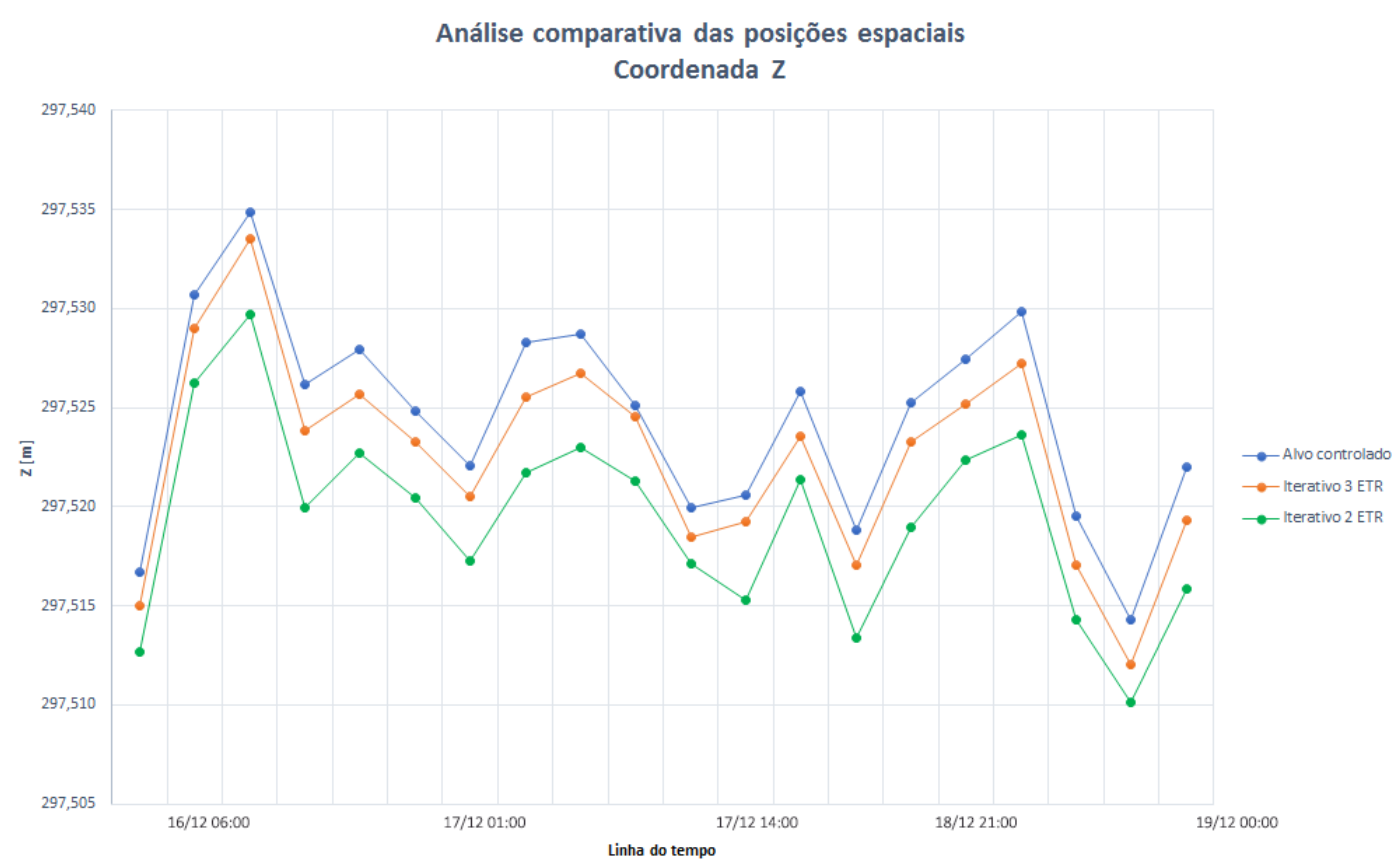

Figura 9.34: Análise comparativa da posição espacial $Z_{P}$. 
A Figura 9.35 mostra a visualização gráfica da análise comparativa da acurácia obtida no eixo $X$ entre o braço robótico e os dois métodos iterativos, com o uso de duas e três estações totais robóticas.

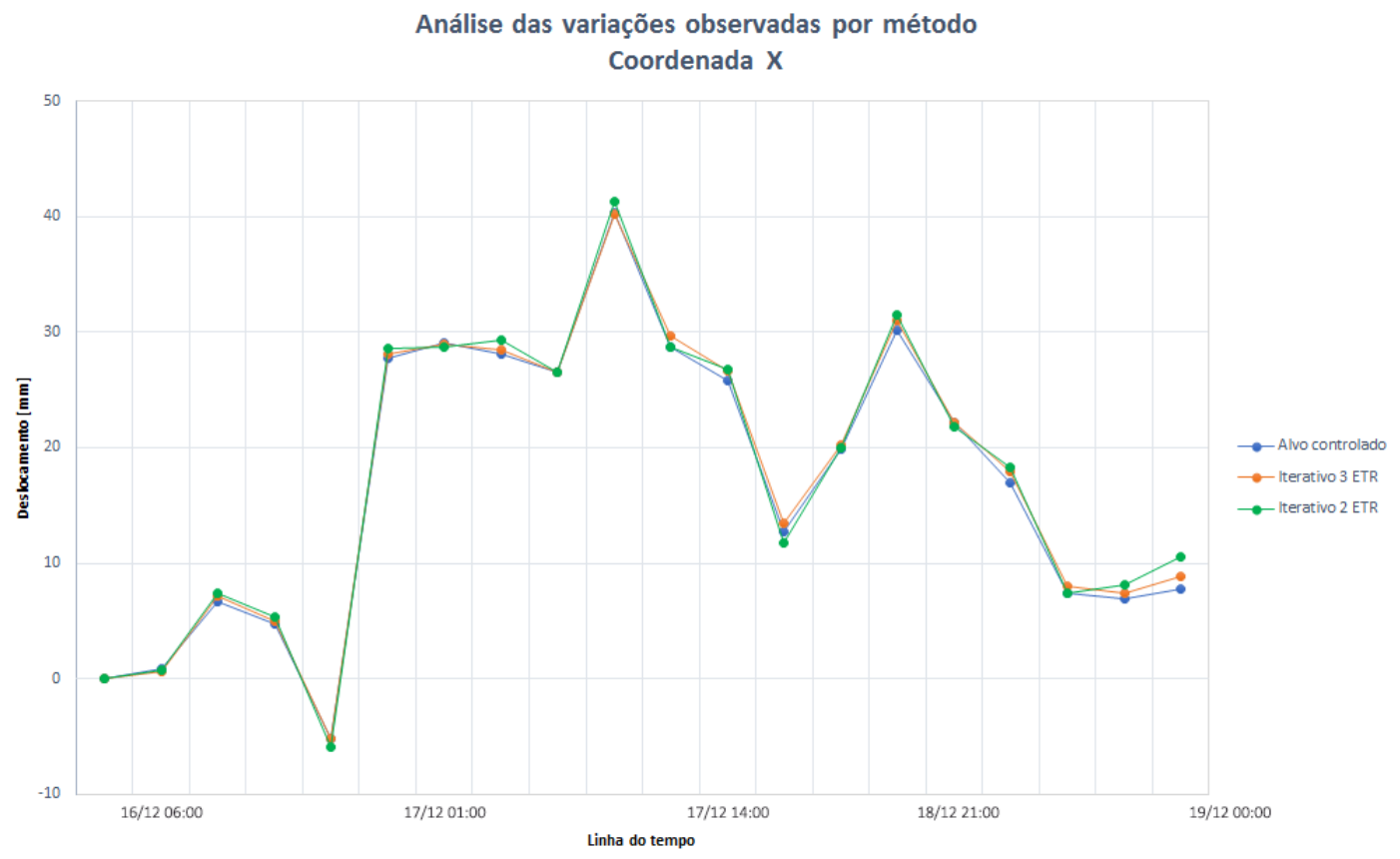

Figura 9.35: Acurácia obtida em $X_{P}$.

A Figura 9.36 mostra a visualização gráfica da análise comparativa da acurácia obtida no eixo $Y$ entre o braço robótico e os dois métodos iterativos, com o uso de duas e três estações totais robóticas.

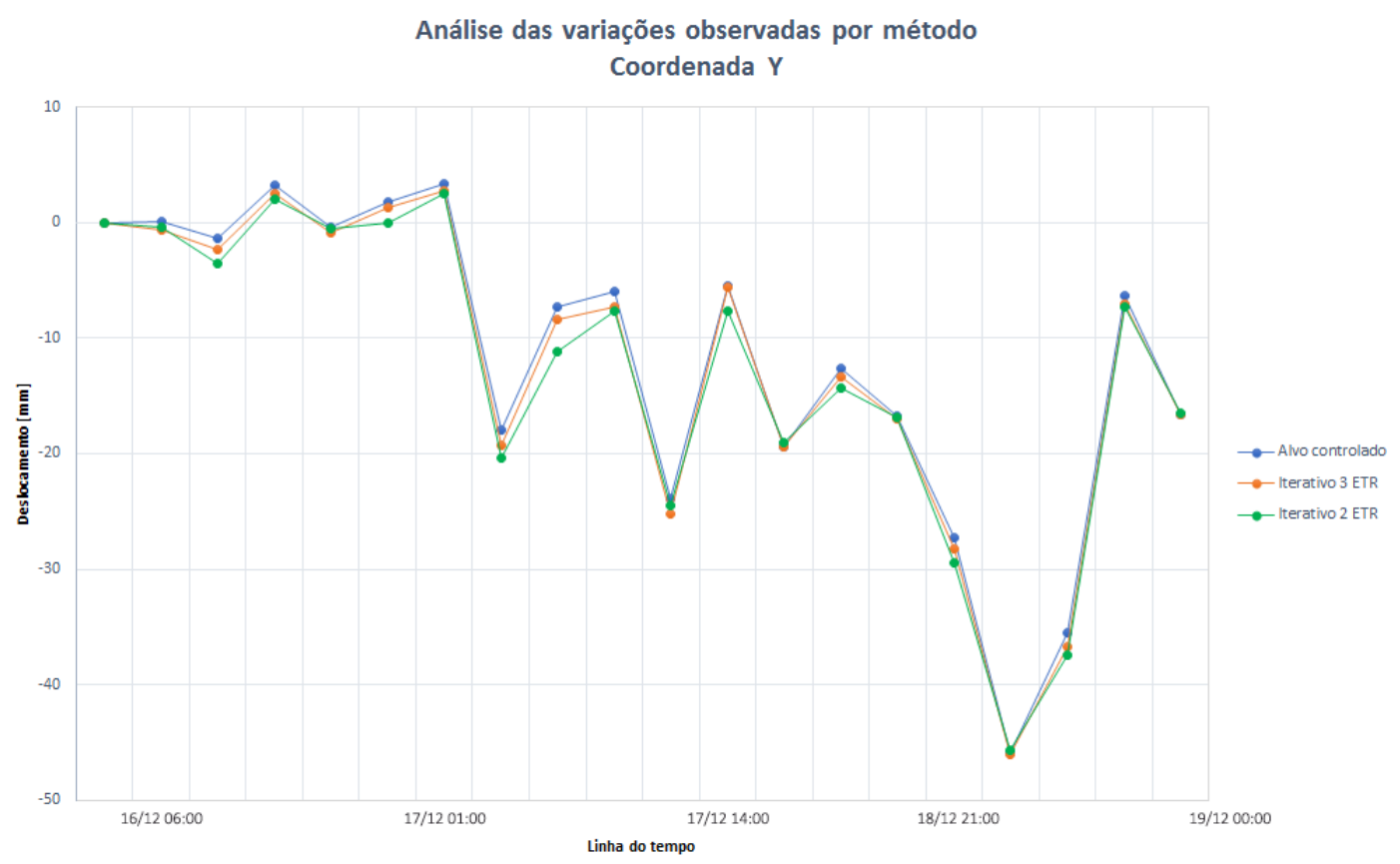

Figura 9.36: Acurácia obtida em $Y_{P}$. 
A Figura 9.37 mostra a visualização gráfica da análise comparativa da acurácia obtida no eixo $Z$ entre o braço robótico e os dois métodos iterativos, com o uso de duas e três estações totais robóticas.

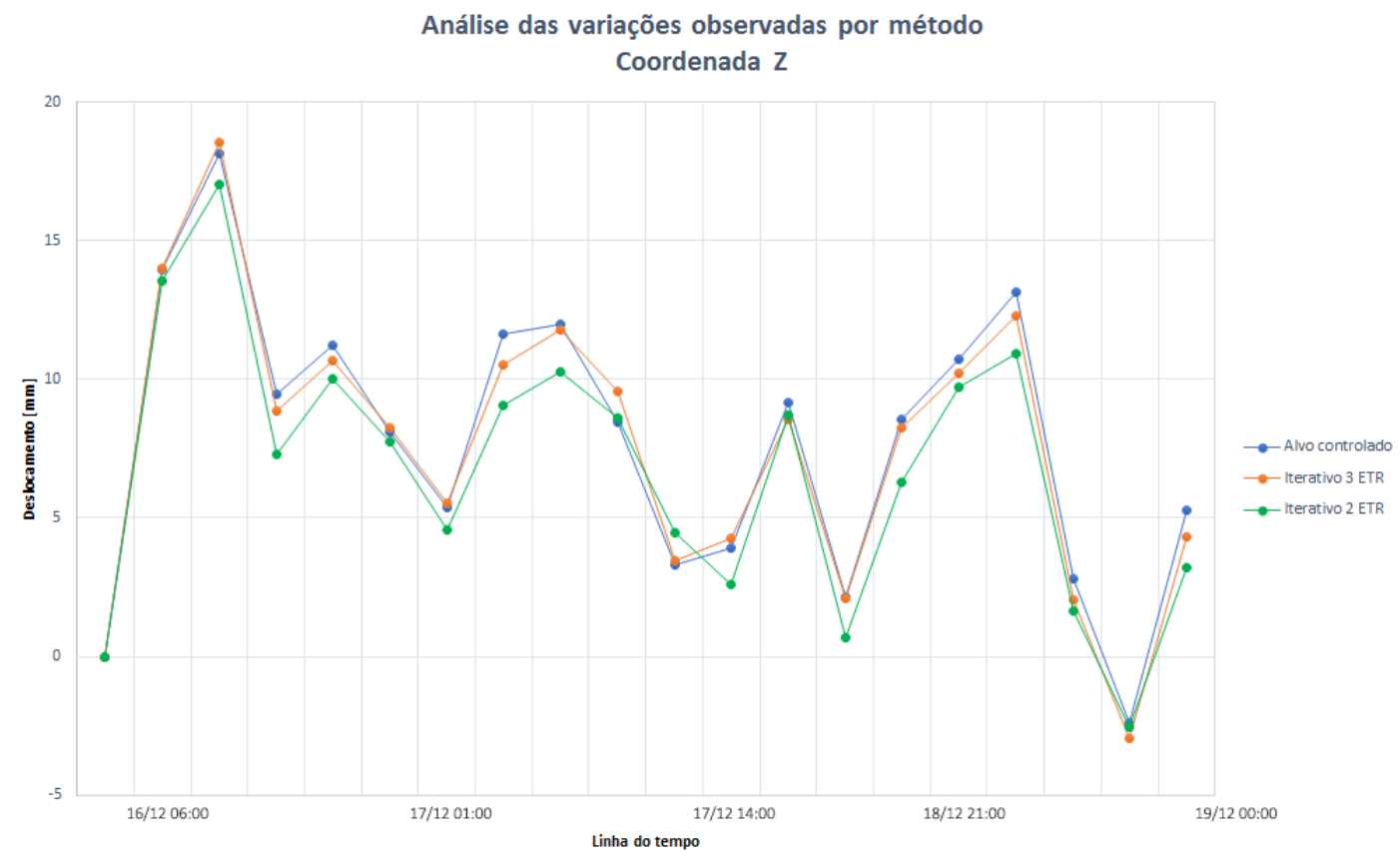

Figura 9.37: Acurácia obtida em $Z_{P}$.

Portanto, observa-se por meio dos gráficos apresentados acima, como os métodos propostos detectam a variação, considerada como absoluta, aplicada pelo braço robótica. Dessa forma, pode-se afirmar que os métodos iterativos de aproximações sucessivas apresentam uma alta acurácia, sendo assim, os métodos que mais se aproximaram da simulação de deslocamentos estruturais. 


\section{Capítulo}

\section{CONCLUSÕES E RECOMENDAÇÕES}

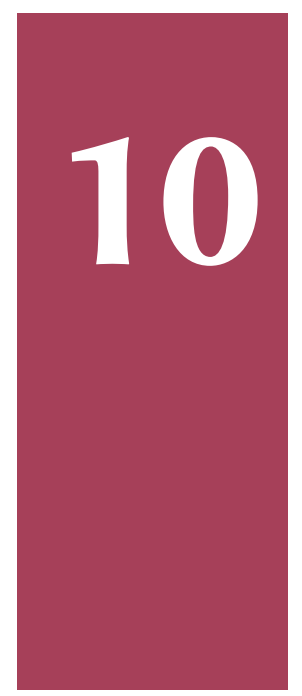

São apresentadas, neste capítulo, as principais conclusões de todas as etapas do desenvolvimento desta tese, com destaque para os métodos de determinação de coordenadas espaciais e para o sistema de monitoramento geodésico de estruturas propostos.

\subsection{Conclusões}

Os resultados obtidos na seção 9.2 e as análises comparativas, realizadas na seção 9.3, considerando todos os métodos estudados, tanto os aqui propostos, quanto os de uso corrente, permitiram obter as seguintes conclusões:

Deve-se enfatizar, primeiramente, que o monitoramento geodésico de estruturas pode ser considerado como um sistema de medição seguro e eficiente, para o controle de deslocamentos de estruturas naturais e artificiais. As técnicas e os instrumentos de medições, bem como os métodos de determinações de coordenadas espaciais disponíveis, garantem qualidade, eficiência e rapidez em monitoramento geodésico de estruturas, com o uso de estações totais robóticas. Ressalta-se, porém, que no caso de distâncias longas, recomenda-se empregar os métodos multipolares com medição de distância.

Em relação aos instrumentos de medição, conforme exposto no Capítulo 3, tem-se observado que o uso de estações totais robóticas, em conjunto com seus acessórios e softwares de gestão, são soluções altamente confiáveis e seguras, principalmente, por possibilitar medições em modo contínuo e cíclico. Nesse sentido, os ensaios realizados tiveram apenas o objetivo de comprovar a possibilidade do uso desse tipo de instrumento, para a validação dos métodos aqui propostos. 
Em relação ao subsistema de medição, primeiramente, considera-se a rede topográfica espacial proposta, que tem embasamento teórico no Capítulo 5. O desenvolvimento desta rede teve, por objetivo, o estabelecimento de uma metodologia para a determinação de coordenadas espaciais com solução $(3 D)$, de forma direta. Isso ocorre devido ao fato de esta rede ter sido desenvolvida por meio de relações geométricas espaciais.

A realização da rede topográfica espacial, nesta tese, validou sua eficiência, de tal forma que se mostrou capaz de integrar um sistema de monitoramento geodésico. Isso ficou comprovado pelas coordenadas espaciais e precisões apresentadas na Tabela 8.4, pelo fato de ela aderir ao teste estatístico bilateral do qui-quadrado, com alto grau de confiabilidade, como mostra a Tabela 8.5 e por, também, apresentar valores milimétricos e submilimétricos relativos à elipse de erros, como pode ser visto na Figura 8.6.

Ainda, deve-se considerar que esta rede foi aplicada, em uma área extensa, com duas bases de medições posicionadas em andares elevados de edifícios e, também, uma outra base posicionada no alto do perfil externo de um edifício de estrutura metálica. Outro fator a ser considerado é a coerência das coordenadas obtidas pelo processo de verificação de estabilidade, aplicado pela recessão espacial. Dessa forma, acredita-se que esta metodologia, aplicada para obtenção de uma rede topográfica espacial, apresenta-se como mais uma opção a ser empregada em monitoramentos geodésicos de estuturas, que exigem de uma rede um referencial absoluto e estável. É importante salientar que esta rede também pode ser aplicada em outras tarefas da Geomática.

Após a rede ser implantada, iniciou-se o processo de monitoramento que possibilitou a determinação das coordenadas espaciais do ponto de monitoramento e, para isso, considerou-se a movimentação exercida pelo braço robótico como absoluta, ou seja, como o valor verdadeiro para as análises comparativas. Este braço robótico foi escolhido, devido ao fato de realizar movimentações espaciais vetoriais com repetibilidade de precisão submilimétrica, como mostram suas especificações técnicas, apresentadas na Figura 3.11. Este braço robótico foi escolhido, também, por possibilitar o acesso ao código fonte e realizar rotinas operacionais para automatizar as movimentações no período e ciclos necessários para este estudo. Além disso, os dados expostos, no Capítulo 9, comprovam sua eficiência na tarefa de simular deslocamentos estruturais. Portanto, pode-se afirmar que esta tecnologia robótica possibilita a realização de estudos que necessitam de controle de deslocamento de alvos e, assim, recomenda-se a sua utilização em simulações de instabilidades estruturais.

Ainda no processo de determinação das coordenadas espaciais do ponto de monitoramento, considerando-se os métodos multipolares sem medição de distância, apresentados na seção 6.2, deve-se ressaltar que todos partem da premissa de que, por meio de medições angulares, determinam-se indiretamente as distâncias inclinadas, sendo assim, todos estes métodos são similares, apenas diferenciando-se nas relações matemáticas. Dessa forma, a distância inclinada estabelecida por estes métodos é geométrica, entretanto, não considera os efeitos atmosféricos 
presentes em uma aplicação prática com longas visadas. Portanto, ao não considerar tais efeitos, estes modelos não possibilitaram aplicar as correções atmosféricas. E, consequentemente, as coordenadas espaciais do ponto de monitoramento, determinadas por estes modelos, não se mostraram com a precisão e a acurácia exigidas em sistemas de monitoramento geodésico de estruturas. Dessa forma, não se recomenda o uso destes métodos em monitoramento geodésico de estruturas com longas visadas. Tal fato ficou comprovado ao se analisar os resultados das coordenadas espaciais e suas respectivas precisões, apresentadas desde a Tabela 9.11 até a Tabela 9.15. Também, de posse das variações detectadas por estes métodos, apresentados entre as Figuras 9.9 e 9.18, é possível mostrar sua ineficácia para sistemas de monitoramento que exigem longas visadas.

Pode-se notar, também, que todos estes métodos detectaram movimentações dentro de tolerâncias centimétricas, como pode ser visto nas análises comparativas extraídas das Tabelas 9.22 a 9.26. Porém, deve-se ressaltar que as experiências práticas mostraram que estes métodos possuem alta aderência nas movimentações de um ponto em deslocamento com visadas curtas, em que o efeito atmosférico é considerado desprezível, mantendo, assim, a distância inclinada geométrica calculada muito próxima da distância inclinada medida por um EDM. Portanto, é possível afirmar que com visadas curtas, estes métodos produzem resultados consistentes. Por fim, recomenda-se aplicar os métodos multipolares sem medição de distância, com linhas de visadas relativamente curtas, em que os efeitos atmosféricos são mínimos.

Para os métodos de determinação de coordenadas espaciais com medição de distância, os testes realizados, no Campus 2, demonstraram que as medições com o uso do método polar é uma solução adequada para a maioria das tarefas da Geomática, em conformidade com Baykal et al. (2005); a inconveniência deste método consiste na impossibilidade de controle dos resultados, como mostra a Tabela 9.9. O método polar também se mostra ineficiente por apresentar suscetibilidade em relação aos erros de medições, uma vez que não dispõe de técnicas de interseção. Por esta razão, recomenda-se para monitoramento, sempre que possível, o uso de métodos multipolares com medição de distâncias, mesmo com modelos matemáticos mais simples, a exemplo da média aritmética entre os valores obtidos por meio das medições de dois ou mais instrumentos. Isso se deve ao fato de inserir mais observações e, assim, oferecer maior redundância aos dados, deixando-os menos suscetíveis aos erros de medições e com maior poder de controle e ajuste, o que, consequentemente, proporciona mais confiança sobre as informações adquiridas. Para isso, nesta pesquisa, foi considerado, também, o valor médio das coordenadas espaciais, obtidas pelo método polar relativo a cada estação total robótica envolvida no processo. Dessa forma, os valores se mostraram mais próximos dos valores de movimentação do braço robótico. Ainda, para os métodos polares, deve-se considerar que a partir de medições multipolares, é possível realizar o ajustamento das observações e, assim, extrair, não só as coordenadas espaciais com valores médios, mas também suas respectivas precisões, como mostra a Tabela 9.10. 
Deve-se considerar também que, conforme apresentado ao longo desta tese, existem outras formas de aplicar o ajustamento das observações ao método multipolar, as quais demandam maior conhecimento matemático e computacional, sendo que uma forma estabelece relação geométrica para gerar a Matriz Jacobiana $A$ e a outra estabelece relação analítica para gerar a mesma matriz. Estes métodos de determinação são embasados no método polar simples e, devido a isso, suas coordenadas espaciais diferem insignificantemente dos métodos que consideram o valor médio do polar. Dessa forma, pode-se afirmar que, para tolerâncias milimétricas, são considerados iguais, pois o processo de ajustamento de cada forma agrega um valor tão pequeno às incógnitas, que as coordenadas espaciais se comportam de modo idêntico. Ressalta-se que a única diferença obtida nos resultados, entre as formas geométrica e analítica de ajustamento, são as precisões. Portanto, reafirma-se que, os métodos clássicos que consideram a metodologia elementar do método polar, sendo eles, multipolar com média, com ajustamento, com minimização com relação geométrica e analítica, ao nível de arredondamentos adotados, com quatro casas decimais, apresentam valores iguais, diferindo nos valores das precisões. Enfatiza-se que, ao aplicar técnicas matemáticas de convergência aos métodos multipolares com medição de distância, estes se mostram com maior qualidade.

Assim, os métodos multipolares com medição de distâncias propostos, que se utilizam de técnicas matemáticas com aproximações sucessivas de forma iterativa, para a determinação de coordenadas espaciais, obtiveram resultados mais eficientes e confiáveis no sentido de que estes apresentam uma melhor aproximação para as coordenadas do alvo em deslocamento, ou seja, maior grau de aderência ao controle absoluto, como mostra o comparativo de acurácias nas Tabela 9.29 e Tabela 9.30. Isso se deve ao fato de que o problema de determinação das coordenadas espaciais do alvo foi reduzido a um problema de determinação de zeros de uma função de duas ou três variáveis, o qual é feito de forma iterativa, após a linearização de um sistema não linear, considerando-se que uma primeira aproximação para o alvo, o chute inicial, foi escolhido para o processo gerar uma sequência de novas aproximações que convergem quadraticamente para a melhor aproximação da coordenada real do alvo. Ressalta-se que a ferramenta matemática utilizada para dizer quando se está de fato próximo o suficiente do alvo é a condição de parada, que é realizada por meio das funções $f$. Por fim, enfatiza-se que as condições que garantem a convergência do método podem ser vistas em Demidovich (1981) e nos resultados obtidos nesta tese, ver a Figura 9.30 para duas estações totais robóticas e a Figura 9.30 para três estações totais robóticas.

Dessa forma, pode-se afirmar que o método proposto, com o uso de duas estações totais robóticas, apresenta-se mais consistente e, quando aplicado, com o uso de mais uma estação total robótica, torna-se possível realizar a média entre os valores obtidos de combinações tomadas duas a duas, o que produz valores mais próximos ainda do valor absoluto gerado pelo braço robótico, que potencializa mais ainda sua eficácia. Ressalta-se que, neste caso de estar utilizando três estações totais robóticas de modo combinado duas a duas, um possível erro em alguma destas estações poderá ser facilmente identificado, uma vez que poderá ser percebido 
na discrepância de informações locais e globais.

Já o método proposto com o uso de três estações totais robóticas é o mais preciso e acurado de todos, isso porque fornece as estimativas de todas as observações das três linhas de visadas para o cálculo das coordenadas do alvo em deslocamento e, ainda assim, como característica do Método de Newton Generalizado, melhora o chute inicial, sendo este aqui adotado como o valor do método polar com média, o qual já é um bom valor e, ainda assim, melhora esses valores, aproximando-o mais ainda do valor absoluto real.

O que diferencia os métodos propostos para esta tese em relação aos métodos estudados, é o fato de que se tiver uma condição inicial dada por algum outro método, por exemplo o método polar, após realizar as iterações sucessivas, a sequência de novas aproximações tende a um valor muito melhor do que os utilizados correntemente, isto pode ser visto por meio da acurácia e das tabelas expostas no Capítulo 9.

Consequentemente, com os cálculos das coordenadas espaciais, pode-se concluir, de forma geral, que os métodos multipolares com medição de distâncias mostraram-se mais consistentes do que os cálculos a partir do método polar e dos métodos multipolares sem medição de distâncias. E, dentre os métodos que inserem também a distância inclinada em sua modelagem, os iterativos com aproximações sucessivas, devido a sua convergência para o valor real mostraram-se mais consistentes ainda.

Em relação aos métodos multipolares com medição de distância, estes se mostraram altamente confiáveis, com um grau de aderência alto em relação às variações ocorridas no deslocamento controlado do alvo, às precisões calculadas e às acurácias obtidas, todas dentro de tolerâncias milimétricas.

Considerando-se os índices de variação em relação ao deslocamento controlado do alvo, pode-se afirmar que os métodos propostos apresentam precisão e acurácia aceitáveis para os trabalhos de monitoramento geodésico ou para outras tarefas da Geomática, como mostram os gráficos apresentados nas Figuras 9.32 à 9.37 .

Deve-se também salientar que os cálculos realizados não consideraram diferentes estratégias de pesos para as medições, mantendo a matriz peso em igualdade com a matriz identidade, o que pode interferir nos resultados obtidos. Também deve-se ponderar que as determinações de coordenadas espaciais foram calculadas em uma rotina computacional em ambiente Python, considerando todos os algarismos gerados e, por fim, arredondados para os algarismos significativos das medições. Por isso, pode haver divergências de resultados, dependendo do modo de cálculo.

Para o subsistema de comunicação, conclui-se que sua operacionalidade é estável e pode ser aplicado em sistemas de monitoramento geodésicos de estruturas com o uso de várias estações totais robóticas e no modo contínuo. Também, mostrou sua potência em efetuar as transmissões de dados em extensa área de atuação, onde não é possível estabelecer comunicação física 
entre os instrumentos de medição e o CORE do sistema. Pelo fato de ele não apresentar nenhuma falha durante todo o processo de testes e validação do sistema. Portanto, recomenda-se o uso deste subsistema de comunicação por ser uma solução viável, eficiente e confiável.

Para o subsistema de processamento e alarme, os resultados obtidos mostraram que o sistema de gerenciamento apresentado neste trabalho foi capaz de gerenciar as informações do banco de dados de forma rápida, efetiva e confiável, além de ser uma plataforma aberta a diversas manipulações, conforme a necessidade do estudo, o que é muito importante para se obter análises gráficas, numéricas ou qualquer outra busca de dados, para embasar tomadas de decisões mais acertadas. Ressalta-se que este subsistema pode servir como ignição no processo de alarmes. Os métodos de convergência apresentados neste trabalho, assim como o processo iterativo, exigem esforço computacional para a execução do modelo. Não existem atualmente softwares dedicados, especificamente, para os modelos propostos, sendo necessária a criação de um novo algoritmo para a demonstração e a validação dos modelos matemáticos.

Em relação ao sistema de monitoramento geodésico de estrutura proposto, pode-se concluir como robusto, eficiente e confiável. O fato de utilizar três estações totais robóticas de alta precisão e de realizar a comunicação de dados, de forma confiável e ininterrupta, possibilita análises confiáveis em tempo real e integração a sistemas de alarmes remotos. Acredita-se que o modelo proposto minimiza a possibilidade de erros e evita que eventuais falhas ou defeitos nas estações totais robóticas interrompam o processo de monitoramento. Trata-se de uma solução com resultados altamente satisfatórios, e sua confiabilidade justifica os investimentos necessários para sua implantação.

Conclui-se, então, que a metodologia proposta para a execução de uma rede topográfica espacial com solução $(3 D)$, de forma direta, é válida para compor uma rede de referência absoluta para o processo de monitoramento. Também, os métodos de determinações de coordenadas espaciais propostos, com técnicas de convergência, são claramente mais acurados e precisos e o fato de considerarem as medições de várias estações totais robóticas os tornam redundantes, mais confiáveis e seguros. Por fim, o sistema de monitoramento geodésico de estruturas desenvolvido atendeu às necessidades destas novas técnicas e proporcionou uma estrutura forte e estável para suas aplicações. Portanto, afirma-se que este sistema de monitoramento geodésico proposto pode ser adotado para aplicação prática de monitoramento e de controle de estruturas artificiais e naturais, com instabilidade. Dessa forma, esta tese oferece mais uma ferramenta da engenharia para a sociedade, objetivando evitar tragédias, prejuízos ambientais e econômicos.

\subsection{Recomendações}

Os estudos e resultados apresentados nesta tese mostram a necessidade de dar continuidade nessa área e linha de pesquisa, dessa forma, são sugeridas recomendações para futuros traba- 
lhos, conforme segue:

Um fator importante a ser analisado nas próximas pesquisas é a influência dos pesos nos resultados. Nos ensaios realizados, aplicou-se um modelo de ponderação convencional, baseado na matriz identidade e, dessa forma, outros modelos deverão ser testados.

Além disso, pretende-se também avaliar a consistência de cada método nas medições conjuntas de várias estações totais robóticas com vários receptores GNSS. Também, pretende-se avaliar a eficiência de cada técnica e seu uso combinado em auscultação estrutural.

Recomenda-se, ainda, estudos com uso de estações totais robóticas em áreas de instabilidade, ou próximas do entorno ou até mesmo parte do corpo estrutural, para que se possa avaliar o desempenho desta solução para os casos em que as estações totais robóticas precisam ser instaladas sobre pontos em movimento conjunto. Este é o caso, por exemplo, de estruturas esbeltas de grandes alturas, e de posicionamentos de fundações fluviais e de plataformas dinâmicas em geral. Para isso, faz-se imprescindível estudar técnicas de medição e de determinação de coordenadas espaciais, por meio de modelos matemáticos de convergência, para recessão espacial.

Outra recomendação, a ser considerada, trata da avaliação do uso de escâneres a laser terrestre, com capacidade de geração de imagens digitais, para o uso no monitoramento geodésico de estruturas. Para isso, devem-se desenvolver técnicas de monitoramento que combinem os resultados das imagens digitais e dos vetores espaciais gerados pelo escâner na avaliação da deformação e deslocamentos de estruturas. Acredita-se que o uso de técnicas de correlação de imagens, associadas aos vetores espaciais, pode produzir modelos espaciais que indiquem a variação da forma e da posição das estruturas monitoradas. Além de avaliar seu uso combinado, com integração em sistemas compostos por variados instrumentos de medição.

Seria importante também, para os próximos trabalhos nesta área, constituir uma equipe interdisciplinar, composta por matemáticos, cientistas da computação e engenheiros interessados na área da Geomática, para a elaboração de outras técnicas e outros modelos matemáticos, para determinação de coordenadas espaciais, que possam ser aplicados a sistemas de monitoramento geodésicos de estruturas.

Por fim, recomenda-se o uso desta tese e dos resultados obtidos para outras linhas de pesquisa nas áreas relacionadas à Geomática, para que, assim, possam ser realizadas novas propostas para a composição de um sistema de monitoramento geodésico de estruturas. 


\section{Referências}

AFENI, T. B.; CAWOOD, F. T. Slope monitoring using total station: what are the challenges and how should these be mitigated? South African fournal of Geomatics, 2013.

ALBA, M.; PONCORONI, M.; SCAIONI, M. Investigation about the accuracy of target measurement for deformation monitoring. The International Archives of the Photogrammetry, Remote Sensing and Spatial Information Sciences, Vol XXXVII, Part B5, 2008.

ALLAN, A. The principles of theodolite intersection systems. Survey Review, 1988.

ALLAN, A. Back to basics (3) theodolite intersection in three dimensions. Survey Review, 1990.

ALLAN, A. Back to basics (4) surveying in three dimensions. Survey Review, 1996.

ALMEIDA, E.; ALEXANDINO, A.; FERNANDES, D. Geodetic Observation in Portuguese Dams. 4th International Conference on Engineering Surveying. Bratislava, Slovakia, 2008.

ANDERSON, J.; MIKHAIL, E. Surveying: Theory and Practice. New York, USA: McGrow-Hill, 1998.

ARMY, D. O. T. Manual 1110-1-1004 Deformation monitoring and control surveying. Washington, DC: US Army Corps of Engineers, 1994.

ARMY, D. O. T. Structural deformation surveying. Washington, DC: US Army Corps of Engineers, 2002.

ARMY, D. O. T. Manual No. 1110-1-1002 Engineering and design: survey markers and monumentation. Washington, DC: US Army Corps of Engineers, 2012.

ASCE. Guidelines for Instrumentation and Measurements for Monitoring Dam Performance. USA: Committee on Instrumentation and Monitoring Dam Performance, sponsored by Hydropower Committee of the Energy Division of the American Society of Civil Engineers (ASCE), 2000. 
ASCE. The Vision for Civil Engineering in 2025. USA: American Society of Civil Engineers (ASCE), 2010.

BAYKAL, O. et al. Accuracy of point layout with polar coordinates. Fournal of surveying engineering, 2005.

BERGMEISTER, K. et al. Monitoring and safety evaluation of existing concrete structures: State of art Report. Lausanne, Switzerland: FIB Federation internationale du beton, 2003.

BESHR, A. Structural deformation monitoring and analysis of highway bridge using accurate geodetic techniques. Engineering, 2015.

BESHR, A.; KALOOP, M. R. Monitoring bridge deformation using auto-correlation adjustment technique for total station observations. Positioning, 2013.

BESHR, A. A.; ELNAGA, I. M. A. Investigating the accuracy of digital levels and reflectorless total stations for purposes of geodetic engineering. Alexandria Engineering fournal, 2012.

BINGLEY, R. Electronic theodolite intersection systems. Tese (Doutorado) - University of Nottingham, Nottingham, 1990.

BIRD, B. Analysis of survey point displacements using total station measurements. British Columbia Institute of Technology, 2009.

BURDEN, R. L.; FAIRES, J. D.; BURDEN, A. M. Numerical Analysis. 10a. ed. Canada: Cengage Learning, 2016.

CHEN, Y. Analysis of deformation surveys - A generalized method. New Brunswick, Canada, 1983.

CHEN, Y.; CHRZANOWSKI, A. An overview of the physical interpretation of deformation measurements. In: Deformation Measurements Workshop. Cambridge. Boston, MA: MIT, 1986.

CHEN, Y.; CHRZANOWSKI, A.; SECORD, J. A strategy for the analysis of the stability of reference points in deformation surveys. CISM fournal, 1990.

CHRZANOWSKI, A. Review of deformation monitoring techniques: notes presented in Wroclaw, Poland. New Brunswick, Canada, 2009.

CHRZANOWSKI, A. et al. Integration of geodetic and geotechnical deformation surveys in the geosciences. Tectonophysics, 1986.

CHRZANOWSKI, A. et al. A comparison of different approaches into the analysis of deformation measurements. In: XVI Congresso Internacional FIG. Montreux, Switzerland: FIG, 1981.

CHRZANOWSKI, A.; SECORD, J. M. Tilt measurement. CRC Press LLC, 2000.

CHRZANOWSKI, A. et al. Report on existing resources, standards, and procedures for precise monitoring and analysis of structural deformation. U.S. Army Topographic Engineering Center, 1992.

CONTE, S.; DE-BOOR, C. Elementary Numerical Analysis, an Algorithmic Approach. São Paulo: McGraw-Hill Book Company, 1980. 
CORMEN, T.; CHARLE, E.; RONALD, L. Introduction to algorithms. Cambridge, Massachusetts: MIT press, 2009.

CRANENBROECK, J. V. State of the art in structural geodetic monitoring solutions for hydro power dams. In: Proceedings of the FIG Working Week. Marrakech, Morocco: FIG, 2011.

DEMIDOVICH, B. Computational Mathematics, Translated from the Russian by George Yankovsky. Moscow, Russia: MIR Publishers, 1981.

EHIGIATOR, I.; ASHRAF, A. B. Modification of geodetic methods for determining the monitoring station coordinates on the surface of cylindrical oil storage tank. Research Journal of Engineering and Applied Sciences (RJEAS), 2012.

EL-DIN, F. Modification of intersection technique in monitoring the engineering structures. Egypt. International fournal of Civil Engineering and Technology (IFCIET), 2014.

ELMASRI, R.; NAVATHE, S. Fundamentals of Database Systems. 7th. ed. USA: Pearson, 2016.

EROL, B. Evaluation of high-precision sensors in structural monitoring. Sensors, 2010.

FANGI, G. Block bundle adjustment for theodolite stations in control networks - the case of the guggenheim museum in bilbao. The International Archives of the Photogrammetry, Remote Sensing and Spatial Information Sciences, 2008.

GHILANI, C. Adjustment Computations: Spatial Data Analysis. 6th. ed. New York, USA: Wiley, 2017.

GHILANI, C. Elementary Surveying: An Introduction to Geomatics. 15th. ed. New York, USA: Pearson, 2018.

ISO17123-1. Optics and Optical Instruments - Field Procedures for Testing Geodetic and Surveying Instruments - Part 1: Theory. Geneva, Switzerland: International Standard Organization, 2002.

ISO17123-2. Optics and Optical Instruments - Field Procedures for Testing Geodetic and Surveying Instruments - Part 2: Levels. Geneva, Switzerland: International Standard Organization, 2001.

ISO17123-3. Optics and Optical Instruments - Field Procedures for Testing Geodetic and Surveying Instruments - Part 3: Theodolites. Geneva, Switzerland: International Standard Organization, 2001.

ISO17123-4. Optics and Optical Instruments - Field Procedures for Testing Geodetic and Surveying Instruments - Part 4: Electro-Optical Distance Meters (EDMInstruments). Geneva, Switzerland: International Standard Organization, 2001.

KHAMEN, H.; FAIG, W. Surveying. 1st. ed. Berlin, Germany: Walter de Gruyter, 1988.

LAMBROU, E.; PANTAZIS, G.; NIKOLITSAS, K. Special marking of 3d networks points for the monitoring of modern constructions. Fournal of Civil Engineering and Architecture, 2011. 2011.

LEICA. Technical reference manual. Heerbrugg, Switzerland: Leica Geosystems AG, 2007.

LEICA. Technical data. Heerbrugg, Switzerland: Leica Geosystems AG, 2009. 
LEICA. Original Accessories, material matters. Heerbrugg, Switzerland: Leica Geosystems AG, 2010.

LIENHART, W.; EHRHART, M.; GRICK, M. High frequent total station measurements for the monitoring of bridge vibrations. Autria, fournal of Applied Geodesy, 2017.

LUTES, J. Automated dam displacement monitoring using a robotic total station. Tese (Doutorado) - Department of Geodesy and Geomatics Engineering, University of New Brunswick, New Brunswick, Canada, 2002.

LUTZ, M. Learning Python. 5th. ed. Canada: O Reilly Media, 2013.

MASCARENHAS, Q. Shell dam horizontal displacement monitoring - comparative study using geodetic measurement, optical plumb and gps technologies. Journal of Applied Geodesy, 2009.

OGUNDARE, J. Implementation of UNB generalized method for the integrated analysis of deformations: a case study. Dissertação (Mestrado) - University of New Brunswick, New Brunswick, Canada, 1990.

OGUNDARE, J. Data analysis and least squares estimation: the geomatics practice. British Columbia, Canada: BCIT Bookstore, 2012.

OGUNDARE, J. Precision Surveying: The Principles and Geomatics Practice. British Columbia, Canada: Wiley, 2015.

OGUNDARE, J. Understanding Least Squares Estimation and Geomatics Data Analysis. British Columbia, Canada: Wiley, 2018.

ONU, C. Current trends on monitoring the deformation of studied constructions. fournal of Geodesy and Cadastre, 2010.

PALAZZO, D. et al. Dynamic monitoring of structures using a robotic total station. In: Proceedings of the Shaping the Change XXIII FIG Congress. Munich, Germany: FIG, 2006.

POLYAK, B. Newtons method and its use in optmization. European fournal of Operation Research, 2007.

RUEGER, J. Electronic Distance Measurement. 4th. ed. Sydney, Australia: Springer, 2012.

SUI, L. et al. Application of 3d laser scanner for monitoring of landslide hazards. The International Archives of the Photogrammetry, Remote Sensing and Spatial Information Sciences, 2008.

SZOSTAK-CHRZANOWSKI, A.; MASSIERA, M. Relation between monitoring and design aspects of large earth dams. In: Proceedings of the 3rd IAG Symposium on Geodesy for Geotechnical and Structural Engineering and 12-th FIG Symposium on Deformation Measurements. Baden, Austria: FIG, 2006.

TSAKIRI, M.; LICHTI, D.; PFEIFER, N. Terrestrial laser scanning for deformation monitoring. In: Proceedings of the 3rd IAG Symposium on Geodesy for Geotechnical and Structural Engineering and 12-th FIG Symposium on Deformation Measurements. Baden, Austria: FIG, 2006. 
WILKINS, R. et al. A fully automated system for monitoring pit wall displacements. In: SME annual meeting. Cincinnati, USA: SME, 2003.

WUNDERLICH, T. A. geodetic monitoring with prismless polar methods. In: FIG Working Week 2001, Bridging the Gap between Cultures. Bratislava, Slovakia: FIG, 2004.

YIGIT, C. O.; INAL, C.; YETKIN, M. Monitoring of tall building dynamic behaviour using precision inclination sensors. In: 13th FIG Symposium on Deformation Measurement and Analysis. Lisbon, Portugal: FIG, 2008. 
Anexos 


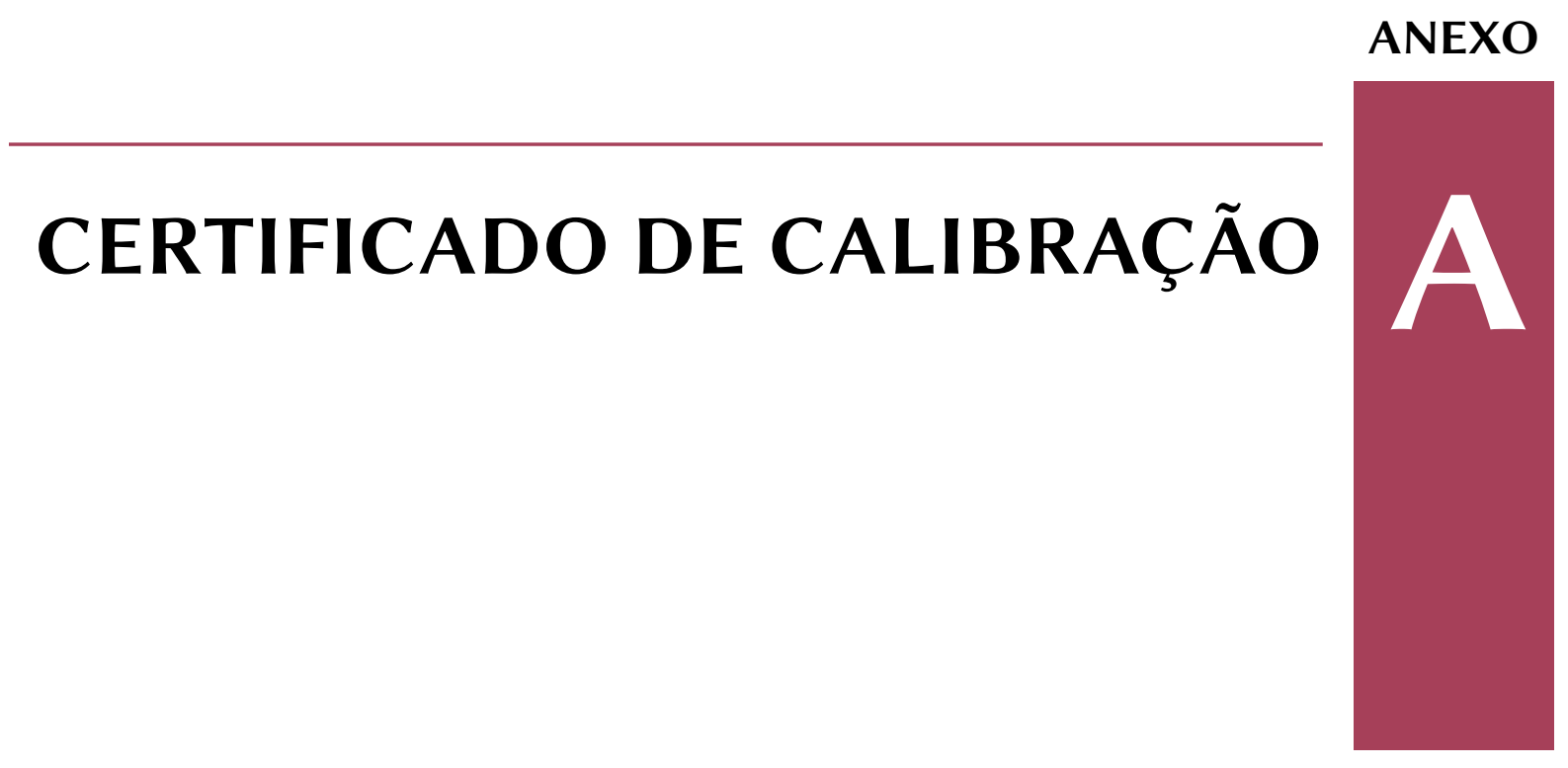

As estações totais robóticas utilizadas na execução desta tese, passaram por processos de verificação e ajuste. Dessa forma, seguem os certificados de calibração de cada instrumento, emitidos pela Braspowertech Soluções Tecnológicas. 


\section{A.1 Certificado de calibração da TCA1201+}

\section{BRASPOWERTECH}

S SOUUOESINTEUGENTES

\section{Braspowertech Soluções Tecnológicas Certificado de Calibração \\ Certificado de calibração sem valores das medições}

$\begin{array}{llll}\begin{array}{l}\text { Equipamento: } \\ \text { № Artigo: }\end{array} & \text { TCA1201+ } & \text { № Certificado: } & 3252034-05122018 \\ \text { № Serie: } & 754306 & \text { Data de inspeção } & 05-12-2018 \\ \text { № Equipamento: } & 240941 & \text { Número do Pedido } & 963-18 \\ \text { Emitido por: } & 3252034 & \\ & \text { Centro de Serviços: } & \\ & \text { Braspowertech Soluções } & \text { Cliente: Diego de Oliveira Martins } \\ & \text { Tecnológicas } & \text { Laboratório de Geomática } \\ & \text { São Carlos, SP } & \text { Departamento de Engenharia de Transportes } \\ & +551634136919 & \text { Escola de Engenharia de São Carlos } \\ & & \text { Universidade de São Paulo (STT/EESC/USP) }\end{array}$

Em conformidade

Certificado de Calibração sem os valores de medição emitido pelo Centro de Serviços da Braspowertech.

\section{Certificado}

Certificamos que o produto descrito foi testado e está em conformidade com as especificações do produto.

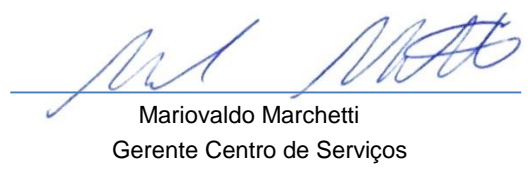

№ certificado 3252034-05122018

Não é permitida a reprodução parcial do presente Certificado sem aprovação formal da entidade emitente 


\section{A.2 Certificado de calibração da TCRP1201+R300}

BRASPOWERTECH

SOLUČ̃ES INTELIGENTES

$(2)^{3}$

\section{Braspowertech Soluções Tecnológicas Certificado de Calibração}

Certificado de calibração sem valores das medições

\begin{tabular}{|c|c|c|}
\hline Equipamento: & TCRP1201 R300 & № Certificado: $\quad 2592148-04122018$ \\
\hline № Artigo: & 737471 & Data de inspeção $\quad 04-12-2018$ \\
\hline № Serie: & 123456 & Número do Pedido \\
\hline № Equipamento: & 2592148 & \\
\hline Emitido por: & $\begin{array}{l}\text { Centro de Serviços: } \\
\text { Braspowertech Soluções } \\
\text { Tecnológicas } \\
\text { São Carlos, SP } \\
\text { +5516 } 34136919\end{array}$ & $\begin{array}{l}\text { Cliente: Diego de Oliveira Martins } \\
\text { Laboratório de Geomática } \\
\text { Departamento de Engenharia de Transportes } \\
\text { Escola de Engenharia de São Carlos } \\
\text { Universidade de São Paulo (STT/EESC/USP) }\end{array}$ \\
\hline
\end{tabular}

Em conformidade

Certificado de Calibração sem os valores de medição emitido pelo Centro de Serviços da Braspowertech.

\section{Certificado}

Certificamos que o produto descrito foi testado e está em conformidade com as especificações do produto.

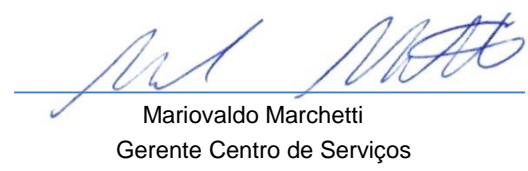

№ certificado 2592148-04122018

Não é permitida a reprodução parcial do presente Certificado sem aprovação formal da entidade emitente

Pagina 1/1
Braspowertech Soluções Tecnológicas

São Carlos, SP - Brasil

+551634136919

www.braspowertech.com.br

Figura A.2: Certificado de calibração da TCRP1201+R300. 


\section{A.3 Certificado de calibração da TCRA1201+R400}

BRASPOWERTECH

SOLUC̄EES INTEUGENTES

\section{Braspowertech Soluções Tecnológicas Certificado de Calibração \\ Certificado de calibração sem valores das medições}

$\begin{array}{llll}\begin{array}{l}\text { Equipamento: } \\ \text { № Artigo: }\end{array} & \text { TCRA1201+R400 } & \text { № Certificado: } & 3277270-06122018 \\ \text { № Serie: } & 754314 & \text { Data de inspeção } & 06-12-2018 \\ \text { № Equipamento: } & 241101 & \text { Número do Pedido } & 964-18 \\ \text { Emitido por: } & 3277270 & \\ & \text { Centro de Serviços: } & \\ & \text { Braspowertech Soluções } & \text { Cliente: Diego de Oliveira Martins } \\ & \text { Tecnológicas } & \text { Laboratório de Geomática } \\ & \text { São Carlos, SP } & \text { Departamento de Engenharia de Transportes } \\ & +551634136919 & \text { Escola de Engenharia de São Carlos } \\ & & \text { Universidade de São Paulo (STT/EESC/USP) }\end{array}$

Em conformidade

Certificado de Calibração sem os valores de medição emitido pelo Centro de Serviços da Braspowertech.

Certificado

Certificamos que o produto descrito foi testado e está em conformidade com as especificações do produto.

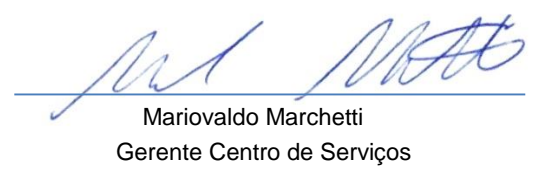

№ certificado $3277270-06122018$

Não é permitida a reprodução parcial do presente Certificado sem aprovação formal da entidade emitente 